\title{
Increased Capacity in WDM \& MDM Networks via Subwavelength Grating \\ Engineering
}

\author{
by \\ Kevan MacKay
}

A thesis submitted to the Faculty of Graduate and Postdoctoral Affairs in partial fulfillment of the requirements for the degree of

Master of Applied Science

in

Electrical \& Computer Engineering

Carleton University Ottawa, Ontario

(C)2021, Kevan MacKay 


\section{Abstract}

As global internet coverage continues to expand, our communication networks must continually evolve to supply the necessary traffic. In order to meet these demands we must develop novel ways to further multiplex optical signals into the same waveguide, through schemes such as wavelength-division-multiplexing (WDM) and mode-division-multiplexing (MDM). The silicon-on-insulator (SOI) platform is a robust and cost-effective choice for designing photonic networks, with a particular focus on high density integration. Within the past decade subwavelength gratings (SWGs) have become a popular avenue of research in silicon photonics, with a variety of applications that include filtering and index engineering. The purpose of this thesis work is to employ silicon-photonic SWGs to enhance the capacity and versatility of WDM/MDM networks.

We first investigate a robust form of highly narrowband SWG Bragg gratings that can be used to design arbitrary transmission spectra with a fine degree of precision. This has the potential to improve the channel-density of WDM networks whose capacity can be limited due to inter-channel spacing. In this work we design and experimentally demonstrate Bragg gratings with sub-nanometer bandwidth sidelobes whose position and extinction ratio can be tailored arbitrarily. Additionally, we have developed a novel fully-etched multimode bend using radially bridged SWG strips. Multimode photonic bends are one of the critical buildingblocks of MDM networks, and a high-capacity bend with a small footprint is essential for further enhancing network density. In this work we design a multimode bend with an effective radius of $10 \mu \mathrm{m}$ that can support up to 5 TE and 4 TM modes on a $340 \mathrm{~nm}$ SOI platform, and experimentally demonstrate a bend for the first 4 TE modes on a $220 \mathrm{~nm}$ SOI platform. 


\section{Acknowledgements}

First and foremost I would like to thank my supervisor, Dr. Winnie N. Ye, for her continued support over the course of this degree. She consistently pushed me to do more than I thought I could, resulting in several opportunities I would otherwise not have had, and I am extremely grateful for that. I want acknowledge the extensive advice and guidance provided by Jens Schmid, Pavel Cheben and Shurui Wang at the NRC. Their assistance was the reason I was able to get experimental results during a year when that would otherwise have been

impossible. I would also like to thank Dusan Gostimirovic, for all the advice and help he provided when I was first learning Lumerical. And finally Sherry, for telling me that I could do this whenever you knew I needed to hear it. 


\section{Contents}

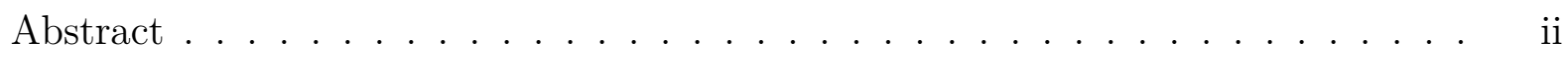

Acknowledgements ...................... iii

List of Figures . . . . . . . . . . . . . . . . . xiv

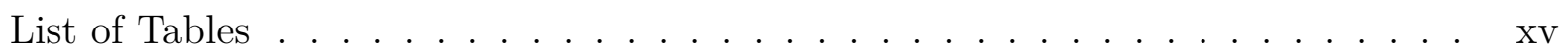

$\begin{array}{ll}\text { Nomenclature } & \text { xvi }\end{array}$

1 Introduction 1

1.1 Multiplexing Network Systems . . . . . . . . . . . . . . . . . . . . . 1

1.1.1 Silicon Photonics . . . . . . . . . . . . . . . . . . 3

1.1.2 Subwavelength Gratings . . . . . . . . . . . . . . . . . 4

1.2 Thesis Organization and Proposed Advancements . . . . . . . . . . . . 4

1.2.1 Arbitrary Bragg Filter Sidelobe Engineering . . . . . . . . . . 5

1.2.2 SWG Engineered Multimode Waveguide Bends . . . . . . . . . . 6

2 Subwavelength Grating Theory \& Simulation Tools $\quad 7$

2.1 Subwavelength Grating Engineering . . . . . . . . . . . . . . 7

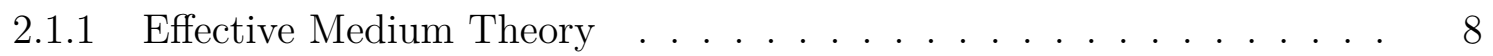

2.1.2 Band Structures and BlochTheorem . . . . . . . . . . . . . . 10

2.2 Simulation Architecture and Tools . . . . . . . . . . . . . . . . . . 13

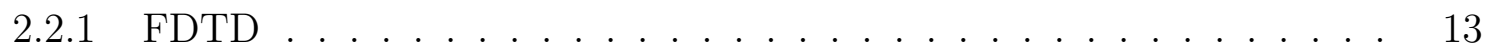

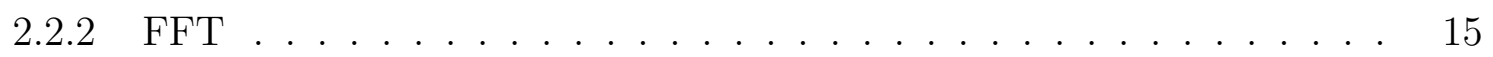


3 Arbitrary Bragg Filter Sidelobe Engineering 16

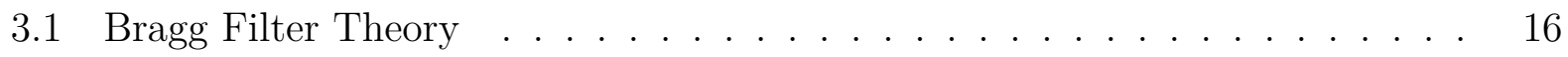

3.1.1 Bragg Condition and Coupled Mode Theory . . . . . . . . . . . 17

3.1 .2 Grating Strength and Bandwidth . . . . . . . . . . . . 18

3.2 Literature Review of Bragg Filters in SOI . . . . . . . . . . 20

3.2.1 Waveguide Geometry Perturbation . . . . . . . . . . . . 20

3.2 .2 Arbitrary Sidelobe Engineering _. . . . . . . . . . . . . . 23

3.3 Proposed Design . . . . . . . . . . . . . . . . . . 26

3.4 Bragg Grating Band Structure . . . . . . . . . . . . . . . 28

3.4.1 Unperturbed Waveguide Characterization . . . . . . . . . . . . . . 29

3.4.2 Relationship between Gap \& Effective Index . . . . . . . . . . . . 31

3.5 Geometry Modulation . . . . . . . . . . . . . . . . . 33

3.5.1 Numerical FFT Simulations . . . . . . . . . . . . . . . . . . 34

3.5 .2 3D FDTD Simulations $\ldots \ldots \ldots \ldots \ldots \ldots$

3.6 Thermal Modulation . . . . . . . . . . . . . . . . . . . . . 42

3.6 .1 Lumerical HEAT Simulations . . . . . . . . . . . . . . . . 43

3.6 .2 3D FDTD Simulations . . . . . . . . . . . . . . . . . 44

3.7 Layout \& Fabrication . . . . . . . . . . . . . . . . . . . 47

3.8 Testing \& Measurement Results . . . . . . . . . . . . . . . . . . . . . . 49

$3.8 .1 \quad$ Propagation Loss $\ldots \ldots \ldots \ldots$

3.8 .2 Passive Filter Performance . . . . . . . . . . . . . . . . . . . 51

3.8 .3 Thermal Filter Performance . . . . . . . . . . . . . . . . 57

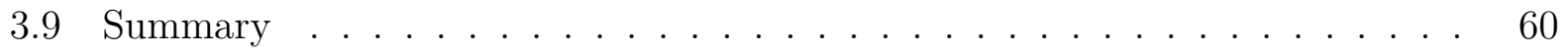


4 SWG Engineered Multimode Waveguide Bends $\quad 61$

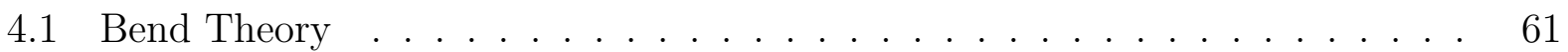

4.1.1 Conformal Transformation of a Bent Waveguide . . . . . . . . . . . . 62

4.1 .2 Sources of Bend Loss . . . . . . . . . . . . . . . . . . . 64

4.2 Literature Review of Multimode Bends in SOI . . . . . . . . . . . . . . . 67

4.2.1 Mode-Converter Assisting Regions . . . . . . . . . . . . . . . . 67

4.2.2 Total-Internal-Reflection via Corner Bends . . . . . . . . . . . . . . 68

4.2.3 Gradient Curvature Bends . . . . . . . . . . . . . . . . . . . . 69

4.2.4 Gradient Index Bends . . . . . . . . . . . . . . . . . . . . 70

4.3 Proposed Design . . . . . . . . . . . . . . . . . . . . . . . 71

4.4 Choice of Bend Curve . . . . . . . . . . . . . . . . . . . 74

4.4.1 Common Bend Alternatives ................. 74

4.4 .2 Optimal Bend . . . . . . . . . . . . . . . . 74

4.5 Waveguide Geometry Cross-section . . . . . . . . . . . . . . . 76

4.5.1 Thickness of $340 \mathrm{~nm} \ldots \ldots \ldots$. . . . . . . . . . . . . . . . . .

4.5.2 Thickness of $220 \mathrm{~nm} \ldots \ldots \ldots \ldots \ldots$

4.6 Mode Delocalization vs Radius . . . . . . . . . . . . . . . . 80

4.7 Index Gradient Engineering . . . . . . . . . . . . . . . . . . 82

4.7.1 Calculation of Optimized Curve . . . . . . . . . . . . . . 82

4.7.2 Trapezoidal SWG Segments . . . . . . . . . . . . . . . 86

4.7 .3 Radial Bridging Strips . . . . . . . . . . . . . . . . . 88

4.7.4 Multimode Fishbone Taper . . . . . . . . . . . . . . . . . . . 89

4.8 3D FDTD Simulations of Bend . . . . . . . . . . . . . . . . . . 93

4.8.1 Band Structure Calculations . . . . . . . . . . . . . . 93 
4.8.2 Optimization Process . . . . . . . . . . . . . . . 96

4.8.3 Optimized Geometries . . . . . . . . . . . . . . 105

4.9 Layout and Fabrication . . . . . . . . . . . . . . . . . 112

4.10 Testing \& Measurement Results . . . . . . . . . . . . . . . . . . . . 114

4.10.1 Grating Coupler Loopback Calibration . . . . . . . . . . . . . . . 115

4.10 .2 (de)MUX Transmission Spectra . . . . . . . . . . . 116

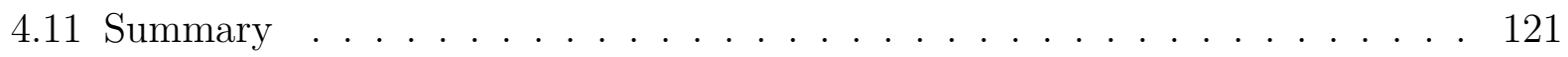

5 Conclusion \& Future Work 122

5.1 Bragg Filters . . . . . . . . . . . . . . . . . . . . . . . 122

5.2 Multimode Bends . . . . . . . . . . . . . . . . . . . 124

5.3 Achievements \& Publications . . . . . . . . . . . . . . . 125

$\begin{array}{lr}\text { List of References } & 126\end{array}$ 


\section{List of Figures}

2.1 a) A one-dimensional subwavelength grating structure, extending infinitely in the $x$ and $y$ dimensions. b) A typical SOI subwavelength grating, finite in $x$ and $y$ and with separate under and upper cladding materials. . . . . . . . . 8

2.2 Diagram showing the relationship between duty-cycle and effective index for

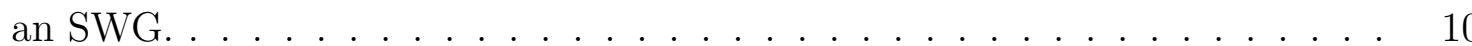

2.3 a) Optical bandstructure response of a finite periodic structure in $z$. b) Physical response of waves travelling in each wavelength regime. . . . . . . . 12

2.4 Example Yee cell from an FDTD mesh. . . . . . . . . . . . . . . . . . . . . . 14

3.1 a) Height corrugation, b) sidewall corrugation, c) cladding modulation. . . . 21

3.2 a) Scanning electron microscope image of cladding-modulated Bragg gratings with an SWG core. b) Measured transmission spectra of the fabricated filters. Reprinted with permission from [23] (C) The Optical Society. . . . . . . . . . . 27

3.3 Layout demonstrating a single cosine modulation of an SWG Bragg grating. 28

3.4 a) $\mathrm{TE}_{0}$ mode bandstructure response. b) $\mathrm{TM}_{0}$ mode bandstructure response. 30

3.5 a) $n_{\text {eff }}$ versus wavelength. b) $n_{g}$ versus wavelength. . . . . . . . . . . . . 30

3.6 a) Dependence of bandwidth and central Bragg wavelength on $g$ for a wire core. b) Calculated values of $\kappa$ and the exponential curves fits. . . . . . . . . 32

3.7 a) Dependence of bandwidth and central Bragg wavelength on $g$ for an SWG core. b) Calculated values of $\kappa$ and the exponential curves fits. . . . . . . . 
3.8 A sample modulation with a single cosine: a) Position of the Bragg segments as a function as distance. b) The corresponding coupling coefficient as a function of distance and the equivalent change in index perturbation. . . . . 35

3.9 Four numerical FFT spectra, each of which has a unique single cosine

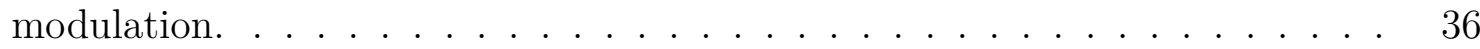

3.10 Two numerical FFT spectra, each of which has a unique double cosine

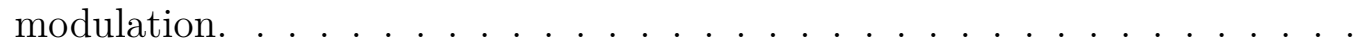

3.11 Reflectance spectra of an unmodulated TM-SWG grating compared to four modulations with different periods. . . . . . . . . . . . .

3.12 Reflectance spectra of an unmodulated TM-SWG grating compared to two modulations with different amplitudes. . . . . . . . . . . . . . . . 40

3.13 Reflectance spectra of an unmodulated grating with and without and offset. 41

3.14 Reflectance spectra of a TM-SWG grating with multiple superimposed cosine modulations of different amplitudes. . . . . . . . . . . . . . . . . . . 42

3.15 a) Periodic heater layout (truncated in length). Blue: Titanium-Tungsten heater element. Pink: Silicon Bragg grating. Brown: Electrode pads. b) Cross-sectional view of fabrication process with heater element. c) Temperature distribution due a voltage boundary condition applied to the simulation . . . . . . . . . . . . . . . . . . . .

3.16 A heater with a spacing of $100 \Lambda_{B}$ was simulated for 16 periods: a) Temperature modulation along the waveguide for different input voltages, b) Resulting FDTD simulation spectra. . . . . . . . . . . . . . 45

3.17 Temperature modulation for a heater with 16 periods and a spacing of a) $50 \Lambda_{B}$, b) $500 \Lambda_{B} \ldots \ldots \ldots \ldots \ldots \ldots \ldots \ldots \ldots \ldots \ldots \ldots \ldots$ 
3.18 SEM pictures of the fabricated Bragg filters: a) A wire-core filter with a constant offset $\xi$ of $200 \mathrm{~nm}$. b) An SWG-core filter with a cosine modulation. c) A zoomed-out view of the previous SWG filter showing a full period of the

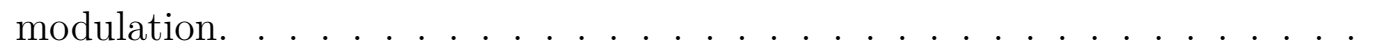

3.19 Average measured TM-input across the wavelength range of 1470-1580, plotted as a function of propagation length. . . . . . . . . . . . 50

3.20 Transmission spectra for unmodulated SWG-filters with TM input. . . . . . 52

3.21 Transmission spectra for SWG gratings with multiple cosine modulations. . . 53

3.22 Transmission spectra for SWG gratings with variable amplitude modulations. 54

3.23 Transmission spectra for unmodulated wire-filters with TM input. . . . . . . 55

3.24 Transmission spectra for wire gratings with multiple cosine modulations. . . 56

3.25 Transmission spectra for wire gratings with variable amplitude modulations. $\quad 57$

3.26 Transmission spectra for a TM SWG filter with a heater element $1.7 \mu \mathrm{m}$ above

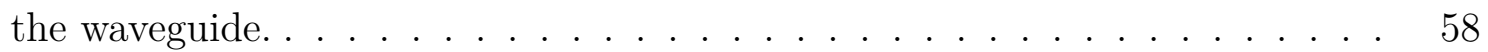

3.27 Transmission spectra for a TM wire filter when no voltage has been supplied versus when $51 \mathrm{~V}$ and $76 \mathrm{~V}$ has been supplied. The heater has a period of $100 \Lambda_{B}$ an approximate resistance of $12.75 k \Omega \ldots \ldots \ldots \ldots$

4.1 a) Index profile of a bent waveguide in $Z$ space. b) Conformal transformation of a bent waveguide index profile such that it is a straight waveguide in $W$

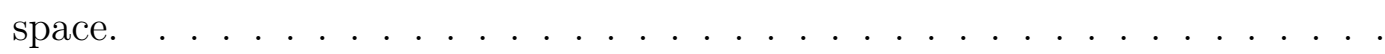

4.2 Bend loss quantified in terms of three regions based on the size of the radius, as defined in [35] @2019 IEEE. . . . . . . . . . . . . . . . . . . . 64 
4.3 a) Graphical demonstration of radiation leakage into the cladding due to the gradient index of a bend. In yellow is the modal field of the fundamental mode, supported only in regions where the local index is greater than $n_{\text {eff }}$. b) Electric field simulation of a $1 \mu \mathrm{m}$ radius bend on a logarithmic scale, showing extreme leakage outwards into the cladding. . . . . . . . . . . . 66

4.4 MC-assisted multimode bends: a) Variable width deformation, reprinted with permission from [37] () The Optical Society. b) Nanohole engineering, reprinted with permission from [39] @ 0 The Optical Society. . . . . . . . . . . 68

4.5 A multimode corner bend that uses total-internal-reflection. Reprinted with permission from $[41]$ @ The Optical Society. . . . . . . . . . . . . . .

4.6 A sample of multimode bends that use gradient curvature and gradient index engineering: a) A Euler bend, reprinted with permission from [43] (C) The Optical Society. b) Partially-etched SWG segments [45] @2020 IEEE. . . .

4.7 A schematic of the multimode bend geometry illustrating the changing dimensions of the trapezoidal segments (red) and the adiabatically tapered radial strips (green) from $\theta=0^{\circ}$ to $\theta=90^{\circ}$. The point of maximum curvature is along line $\mathrm{AA}^{\prime}$ at $\theta=45^{\circ} \ldots \ldots \ldots \ldots \ldots \ldots \ldots$

4.8 Dispersion sweep of a $340 \mathrm{~nm}$ tall silicon waveguide showing the first $5 \mathrm{TE}$ and first 5 TM modes. . . . . . . . . . . . . . . . . . . 77

4.9 Dispersion sweep of a $340 \mathrm{~nm}$ tall silicon waveguide at the design width of

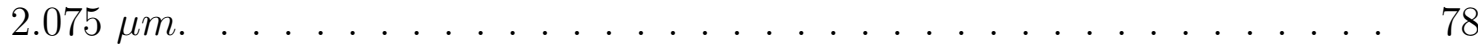

4.10 Dispersion sweep of a $220 \mathrm{~nm}$ tall silicon waveguide showing the first $5 \mathrm{TE}$ and first $5 \mathrm{TM}$ modes. . . . . . . . . . . . . . . . . . 
4.11 Dispersion sweep of a $220 \mathrm{~nm}$ tall silicon waveguide at the design width of $2.075 \mu m . \ldots \ldots \ldots \ldots \ldots \ldots \ldots$

4.12 Mode overlap as a function of radius for a $2.075 \mu \mathrm{m}$ by $220 \mathrm{~nm}$ waveguide for the first four TE modes. . . . . . . . . . . . . . . . . . . . .

4.13 Mode overlap as a function of radius for a $2.075 \mu \mathrm{m}$ by $220 \mathrm{~nm}$ waveguide for the first five TE and TM modes. . . . . . . . . . . . . . . . . .

4.14 a) Optimized curve parameterized by $\theta$. b) The optimized curve with equidistant spacing of $220 \mathrm{~nm}$. A conventional circular bend is shown for

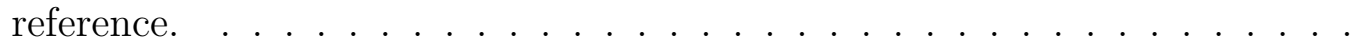

4.15 a) Curvature at each individual SWG segment. b) Rotation angle of each individual SWG segment. . . . . . . . . . . . . . . . . . .

4.16 a) A standard rectangular SWG. b) A trapezoidal SWG with different dutycycles on each side. . . . . . . . . . . . . . . . . . . . . 86

4.17 Variation of inner and outer duty-cycles of the trapezoidal SWG segments. . 87

4.18 a) Scale diagram of eight radial bridging strips with tapered widths. b) Change in strip width as a function of angle. . . . . . . . . . . . . . . . . . . 89

4.19 A standard single-mode fishbone taper between a solid waveguide and SWG. $\quad 90$

4.20 A demonstration of a conventional fishbone taper and a multimode equivalent fishbone taper. . . . . . . . . . . . . . . . . . .

4.21 The designed multimode fishbone taper. The solid waveguide transitions into the SWG bend by tapering the size of the etched holes up from the minimum feature size to the size at the input of the bend. . . . . . . . . . . . . . . 92

4.22 Wavelength response of $n_{\text {eff }}$ vs wavelength for the $220 \mathrm{~nm}$ variant: a) $\mathrm{TE}_{0}$, b) $\mathrm{TE}_{1}$, c) $\left.\mathrm{TE}_{2}, \mathrm{~d}\right) \mathrm{TE}_{3} . \ldots \ldots \ldots \ldots$ 
4.23 Wavelength response of $n_{\text {eff }}$ vs wavelength for the $340 \mathrm{~nm}$ variant: a) $\mathrm{TE}_{0}$, b) $\mathrm{TE}_{1}$, c) $\mathrm{TE}_{2}$, d) $\mathrm{TE}_{3}$, e) $\mathrm{TE}_{4}$, f) $\mathrm{TM}_{0}$, g) $\mathrm{TM}_{1}$, h) $\mathrm{TM}_{2}$, i) $\mathrm{TM}_{3}$, j) $\mathrm{TM}_{4} . \quad 95$

4.24 Flowchart demonstrating the workflow of the multimode bend optimization

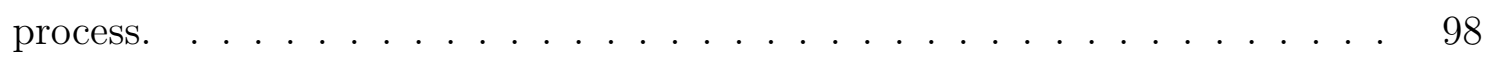

4.25 The $\mathrm{TM}_{1}$ input injected into a $340 \mathrm{~nm}$ bend with a) excellent overlap, b) poor overlap. . . . . . . . . . . . . . . . . . . . . . 100

4.26 Parameter sweep evaluating mode overlap at the output of the $340 \mathrm{~nm}$ bend variant: a) $\mathrm{TE}_{0}$, b) $\mathrm{TE}_{1}$, c) $\mathrm{TE}_{2}$, d) $\mathrm{TE}_{3}$, e) $\mathrm{TE}_{4} \ldots \ldots \ldots 1$

4.27 Parameter sweep evaluating mode overlap at the output of the $340 \mathrm{~nm}$ bend variant: a) $\mathrm{TM}_{0}$, b) $\mathrm{TM}_{1}$, c) $\mathrm{TM}_{2}$, d) $\mathrm{TM}_{3}$, e) $\mathrm{TM}_{4} \ldots \ldots$. . . . . . . . . 102

4.28 Parameter sweep evaluating mode overlap at the output of the $220 \mathrm{~nm}$ bend variant: a) $\mathrm{TE}_{0}$, b) $\mathrm{TE}_{1}$, c) $\mathrm{TE}_{2}$, d) $\mathrm{TE}_{3} \ldots \ldots \ldots \ldots . \ldots \ldots$

4.29 Mode overlap optimization results for $340 \mathrm{~nm}$ bend variant: a) $\mathrm{TE}_{0}$, b) $\mathrm{TE}_{1}$, c) $\mathrm{TE}_{2}$, d) $\mathrm{TE}_{3}$, e) $\mathrm{TE}_{4}$, f) $\mathrm{TM}_{0}$, g) $\mathrm{TM}_{1}$, h) $\mathrm{TM}_{2}$, i) $\mathrm{TM}_{3}$, j) $\mathrm{TM}_{4} \ldots 105$

4.30 Mode overlap optimization results for $220 \mathrm{~nm}$ bend variant: a) $\mathrm{TE}_{0}$, b) $\mathrm{TE}_{1}$,

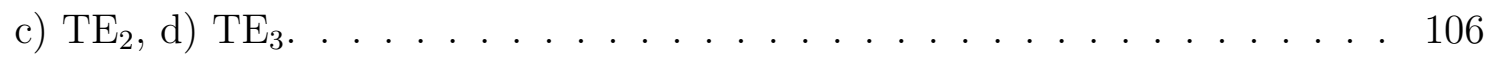

4.31 Transmission spectra for a $340 \mathrm{~nm}$ bend without a taper: a) $\mathrm{TE}_{0}$, b) $\mathrm{TE}_{1}$, c) $\mathrm{TE}_{2}$, d) $\mathrm{TE}_{3}$, e) $\mathrm{TE}_{4}$. With a $5 \mu m$ taper: f) $\left.\left.\mathrm{TE}_{0}, \mathrm{~g}\right) \mathrm{TE}_{1}, \mathrm{~h}\right) \mathrm{TE}_{2}$, i) $\mathrm{TE}_{3}$, j)

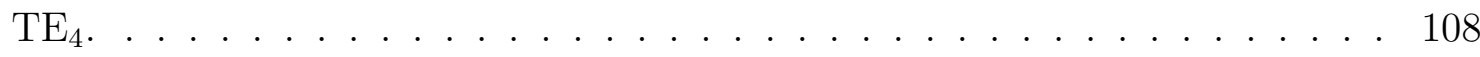

4.32 Transmission spectra for a $340 \mathrm{~nm}$ bend without a taper: a) $\mathrm{TM}_{0}$, b) $\mathrm{TM}_{1}$, c) $\mathrm{TM}_{2}$, d) $\mathrm{TM}_{3}$, e) $\mathrm{TM}_{4}$. With a $5 \mu m$ taper: f) $\mathrm{TM}_{0}$, g) $\mathrm{TM}_{1}$, h) $\mathrm{TM}_{2}$, i)

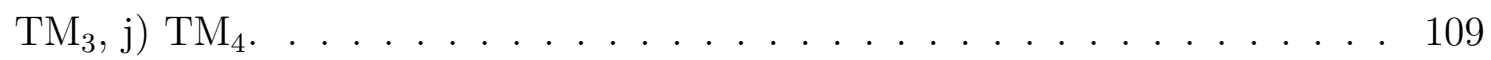

4.33 a) to d) Transmission spectra for the $\mathrm{TE}_{0-3}$ modes without a taper. e) to $\mathrm{h}$ ) Transmission spectra for the $\mathrm{TE}_{0-3}$ modes with a $5 \mu m$ long taper. $\ldots . . .110$ 
4.34 Layout diagram of one half of a (de)MUX circuit. It consists of four input grating couplers, each one coupling a different TE mode into the bus waveguide using adiabatic tapers. The multimode bends can be incorporated into this circuit using two in an S-bend configuration. . . . . . 113

4.35 SEM photographs of the fabricated (de)MUX circuit for testing the multimode bend. a) The (de)MUX circuit, b) the multimode bend, and c) a zoomed-in picture of the bend. . . . . . . . . . . . . . . . . . . . . . . . . 115

4.36 Transmission spectrum response for a grating coupler loop of length $1 \mathrm{~mm}$. Measured data is shown in blue and the curve fit is shown in red. . . . . . . 116

4.37 Measured transmission spectra for a (de)MUX with a straight waveguide: a) $\left.\mathrm{TE}_{0}, \mathrm{~b}\right) \mathrm{TE}_{1}$, c) $\left.\mathrm{TE}_{2}, \mathrm{~d}\right) \mathrm{TE}_{3} \ldots \ldots \ldots \ldots \ldots \ldots \ldots$

4.38 Measured transmission spectra for a (de)MUX with a standard circular bend:

a) $\mathrm{TE}_{0}$, b) $\mathrm{TE}_{1}$, c) $\left.\mathrm{TE}_{2}, \mathrm{~d}\right) \mathrm{TE}_{3} \ldots \ldots \ldots \ldots \ldots \ldots \ldots \ldots$

4.39 Measured transmission spectra for a (de)MUX with a multimode S-bend: a) $\left.\mathrm{TE}_{0}, \mathrm{~b}\right) \mathrm{TE}_{1}$, c) $\left.\mathrm{TE}_{2}, \mathrm{~d}\right) \mathrm{TE}_{3} \ldots \ldots \ldots \ldots \ldots$ 


\section{List of Tables}

3.1 Summary of the key Bragg parameters for six $g$ values and both TE and TM

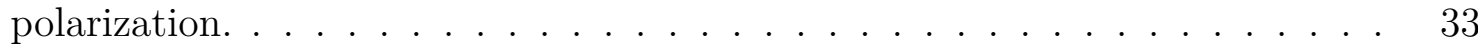

4.1 Optimized geometries for the $340 \mathrm{~nm}$ and $220 \mathrm{~nm}$ multimode bends. The initial duty-cycle is $60 \%$ for both cases and the starting strip width is $170 \mathrm{~nm}$ for both cases. . . . . . . . . . . . . . . . . . . . 105

4.2 Maximum excess loss and intermodal crosstalk for the $340 \mathrm{~nm}$ bend variant with and without a $5 \mu m$ taper. . . . . . . . . . . . . . 111

4.3 Maximum excess loss and intermodal crosstalk for the $220 \mathrm{~nm}$ bend variant with and without a $5 \mu m$ taper. . . . . . . . . . . . . . 111

4.4 Dimensions of the fabricated (de)MUX circuit. . . . . . . . . . . . . . 113

4.5 Performance comparison between (de)MUX with a straight waveguide and the SWG multimode bends. . . . . . . . . . . . . . . . . . . . . . . . . . 119 


\section{Nomenclature}

\section{List of Abbreviations}

WDM: Wavelength Division Multiplexing

MDM: Mode Division Multiplexing

SOI: Silicon on Insulator

SWG: Subwavelength Grating

CMOS: Complementary Metal Oxide Semiconductor

PIC: Photonic Integrated Circuit

PDM: Polarization Division Multiplexing

(de)MUX: (de)Multiplexer

TE: Transverse Electric

TM: Transverse Magnetic

FDTD: Finite Difference Time-Domain

FDM: Finite Difference Eigenmode

FFT: Fast Fourier Transform

FEM: Finite Element Method 
EME: Eigenmode Expansion Method

CMT: Coupled Mode Theory

MC: Mode-Converter

PMMA: Polymethyl Methacrylate

TIR: Total-Internal-Reflection

MMI: Multimode Interferometer

FOM: Figure of Merit

EL: Excess Loss

CT: Intermodal Crosstalk

DUT: Device Under Test

\section{List of Symbols}

$\Lambda_{S W G}:$ SWG Period

$a / \Lambda:$ Duty-Cycle

$n_{\text {eff }}:$ Effective Index

$n_{g}: \quad$ Group Index

$n_{\|}: \quad$ Parallel Effective Index

$n_{\perp}: \quad$ Perpendicular Effective Index

$\lambda$ : Wavelength 
$\omega: \quad$ Frequency

$K: \quad$ Bloch Wavenumber

$c: \quad$ Speed of Light in Vacuum

$E: \quad$ Electric Field Strength

$H: \quad$ Magnetic Field Strength

$\Delta n: \quad$ Index Modulation

$\Lambda_{B}: \quad$ Bragg Period

$\lambda_{B}: \quad$ Bragg Wavelength

$\beta_{0}: \quad$ Wavevector

$\kappa$ : Coupling Coefficient

$\Delta \lambda: \quad$ Filter Bandwidth

$R: \quad$ Reflectance

T: $\quad$ Transmittance

g: $\quad$ Bragg Segment Gap

$L: \quad$ Filter Length

$g_{i}: \quad$ Bragg Segment Modulation Amplitude

$\Lambda_{i}$ : Bragg Segment Modulation Period

$\Delta \lambda_{i}: \quad$ Sidelobe Inter-Peak Spacing 
$\xi: \quad$ Bragg Segment Offset

$v_{p}: \quad$ Phase Velocity

$v_{g}: \quad$ Group Velocity

$d n / d T:$ Thermo-Optic Coefficient

$\Delta T:$ Maximum Temperature Swing

$T_{a v}: \quad$ Average Background Temperature

$n_{\text {con }}(u)$ : Conformal Transformation of a Bent Waveguide Index

$\alpha_{r}: \quad$ Radiation Bend Loss

$\alpha_{s w}: \quad$ Sidewall Scattering Loss

$\alpha_{m}: \quad$ Mode Mismatch Loss

$R_{e f f}:$ Effective Radius

$\eta_{1} / \eta_{2}$ : Inner to Outer Duty-Cycle Ratio

$C: \quad$ Curvature

$L_{c}: \quad$ Coupling Length

$T_{i j}$ : Power Transmission Between Mode $i$ Input and Mode $j$ Output 


\section{Chapter 1}

\section{Introduction}

\section{$1.1 \quad$ Multiplexing Network Systems}

In 2016 the global internet traffic was estimated to be 3.2 exabytes per day, with a projected traffic in 2021 of 9.1 exabytes per day, a 3-fold increase [1]. Between 2016 and 2021 the proportion of the world's population using the internet has grown from $44 \%$ to $58 \%$, with projections showing $66 \%$ of the world's population by 2023 . As the demand for faster connections increases, as well as the number of global internet users, the bandwidth of optical interconnects will have to scale alongside it. Multiplexing and de-multiplexing systems ((de)MUX) form the backbone of high bandwidth optical interconnects, allowing potentially hundreds of data channels to travel through the same bus-waveguide before being filtered into separate outputs. Multiplexing vastly reduces the amount of hardware (and subsequently the financial cost) required to operate a given network, whether it be a long-haul optical fiber or a photonic integrated-circuit. Three of the most commonly used forms of multiplexing architectures are wavelength-division-multiplexing (WDM), mode-division-multiplexing (MDM), and polarization-division-multiplexing (PDM). They can be used independently or as hybrid-systems, multiplying the net transmission rate.

WDM architectures encode different data channels onto a single mode using equally spaced wavelengths supplied by dozens of tunable semiconductor lasers. For instance, in 
2002 a single-mode fiber could support $12.5 \mathrm{GHz}$ spacing within the C-band to create a 512-channel WDM system [2]. This WDM system increased the fiber's signal capacity from $2.5 \mathrm{~Gb} / \mathrm{s}$ up to $1.28 \mathrm{~Tb} / \mathrm{s}$. More recently WDM systems have been enhanced with phaseshift keying and PDM. In 2021 differential quadrature phase-shift keying was used to combine three $40 \mathrm{~Gb} / \mathrm{s}$ signals onto a single wavelength for a net capacity of $120 \mathrm{~Gb} / \mathrm{s}$ per channel [3]. The total 32-channel system achieved a net signal capacity of $3.84 \mathrm{~Tb} / \mathrm{s}$. As the gain window for optical amplifiers is only a few tens of nanometers, high-capacity WDM systems require a dense wavelength spacing. The challenge with decreasing the channel-spacing is that it becomes increasingly difficult to effectively filter adjacent channels while minimizing interchannel crosstalk. Developing a precise way to engineer narrowband filters of arbitrary spacing and extinction ratio is a major stepping-stone to increasing the capacity of WDM networks.

MDM architectures encode different data channels onto separate optical modes, and PDM systems can potentially double this capacity by encoding onto separate TE and TM polarizations. Just last year the first petabit/s optical interconnect was demonstrated using a 15-mode optical fiber [4]. Common implementations of MDM systems involve several adiabatic directional couplers that couple fundamental mode inputs into higher order modes of a bus waveguide. These systems have the advantages of requiring only a single wavelength source and driver. One critical challenge facing MDM systems is the device footprint. For instance, a large bus-waveguide of width $3.5 \mu \mathrm{m}$ is required to support the first $10 \mathrm{TE}$ mode orders. Furthermore, a waveguide of this size would require a bend radius on the order millimeters in order to preserve the symmetry of higher order mode profiles, as well as to minimize radiation loss. Developing high-capacity multimode bends with micron-scale radii is a major goal that would significantly improve the versatility and density of MDM 
networks.

\subsubsection{Silicon Photonics}

The birth of the modern field of photonics coincides with the invention of the laser in the year 1960 [5]. The ability to reliably produce coherent light sources made it possible to harness photons for use in signal processing, amplification, and sensing. This was soon followed by the critical innovations of light-emitting-diodes and optical fibers, both of which eventually paved the way for the mass communication networks that power the modern internet. Unlike metallic interconnects, which are inherently bandwidth-limited to transmission speeds in the $\mathrm{Gb} / \mathrm{s}$ range [6], optical interconnects have demonstrated $\mathrm{Tb} / \mathrm{s}$ speeds at significantly lower power and are future of high capacity communication networks. However, photonic systems have some inherent disadvantages, namely in regards to data-storage and logic computation [7]. Silicon photonics is a rapidly growing area of research that aims to solve these issues through the monolithic integration of electronic and photonic components into a single chip [8].

The silicon-on-insulator (SOI) photonics platform is compatible with the existing complementary-metal-oxide semiconductor (CMOS) fabrication infrastructure, rendering it extremely cost-effective. It leverages several decades of research and development in microelectronics by repurposing it for the fabrication of photonic-integrated circuits (PICs). Although SOI as a platform was first developed in the late 1980s, there has been rapid growth in the field of photonics since 2004 due to significant investments being made into SOI CMOS foundries [9].

Silicon has many attractive features as a photonic material platform. Silicon, as well as its native oxide, are transparent within telecommunications wavelength range 1.3-1.6 
$\mu m$. Silicon and silicon dioxide have a high index contrast $\left(n_{\mathrm{Si}}=3.48, n_{\mathrm{SiO} 2}=1.44\right)$ that is ideal for waveguides with high confinement, allowing smaller overall device footprints. As a passive medium, SOI waveguides are useful for a variety of passive devices such as directional couplers, Y-branch splitters, Bragg filters and ring resonators. A famous 1987 paper [10] demonstrated the plasma dispersion effect in silicon, showing that silicon's refractive index can be altered through the injection of charge carriers. This paved the way for research into further modulation techniques such as the thermo-optic, Kerr, and Franz-Keldysh effects, leading to the development of active devices such as modulators and switches.

\subsubsection{Subwavelength Gratings}

Since their first implementation in SOI in 2010 [11], subwavelength gratings (SWGs) have proven to be one of the most active areas of research within silicon photonics. With increased resolution thanks to electron-beam lithography processes, subwavelength gratings have become a practical design technique in many photonic circuits, adding an extra degree of freedom when tailoring the refractive index profile. SWGs have found use as waveguides, tapers, directional couplers, filters, bends, and waveguide crossings, among many others. In this thesis work we demonstrate how subwavelength gratings can be applied to both WDM and MDM applications to further increase their signal capacity.

\subsection{Thesis Organization and Proposed Advancements}

This thesis is organized into three main chapters, the first of which is a theoretical overview of SWGs and their applications within silicon photonics. The simulation techniques and tools used throughout this thesis will be introduced as well. The remaining two chapters focus 
on distinct projects that relate to WDM and MDM systems. Chapter 3 covers the design, simulation, and fabrication of narrowband Bragg filters that can be engineering to produce an arbitrary transmission spectrum, ideal for high capacity WDM networks. Chapter 4 covers the design, simulation and fabrication of a fully-etched multimode bend design that is an essential building-block of high capacity MDM systems. Following this a concluding chapter will summarize the advancements in addition to a discussion of potential future work. A more detailed overview of chapters 3 and 4 is included below.

\subsubsection{Arbitrary Bragg Filter Sidelobe Engineering}

Bragg filters have been implemented in optical fibers since the 1970's [12], and in the past decade they have been successfully translated to the SOI platform. Bragg filters allow for add/drop multiplexing of specific wavelength channels. Narrowband Bragg filters have been difficult to implement in SOI due to the high index contrast of the medium, but designs that utilize SWG segments have allowed for the weak index modulation necessary for narrowband Bragg filters. In this thesis we present a Bragg filter with a weakly modulated cosine envelope that can be used to design arbitrary transmission spectrums with narrow bandwidths (hundreds of picometers). This versatile cosine envelope can be used to tailor the extinction ratio, bandwidth, and peak spacing of a Bragg filter, allowing for dense WDM systems with increased signal capacity. This cosine envelope can be implemented either through the spatial location of SWG segments, or by using a dynamic periodic heating profile. 


\subsubsection{SWG Engineered Multimode Waveguide Bends}

Silicon photonics has focused on single-mode applications for many years with great success. Small radius SOI bends have been fairly easy to implement because of the high confinement afforded by the high index contrast of the platform. Higher order mode division multiplexing (MDM) has been explored in large straight waveguides, but one key limitation of multimode SOI systems is that photonic bends are extremely difficult to implement due to the asymmetry of the higher order mode profiles [13]. $4^{\text {th }}$ order modes require bend radii in the millimeter range, which is orders of magnitude higher than the footprint of single-mode photonic circuits. We propose a polarization-independent photonic bend that uses radially bridged-SWG segments and an optimized curve shape to allow for 5 TE and 4 TM modes to propagate. The SWG segments are inherently broadband, allowing the bend to be used across a sizeable range of wavelengths in the telecomm band. 


\section{Chapter 2}

\section{Subwavelength Grating Theory \& Simulation Tools}

\subsection{Subwavelength Grating Engineering}

A subwavelength grating (SWG) is a periodic structure whose pitch $(\Lambda)$ is sufficiently smaller than the wavelength of electromagnetic radiation interacting with the structure (see Fig. 2.1), such that the diffraction of the wave is suppressed. SWGs are a concept that have been investigated as far back as the pioneering days of radio waves [14], and have continued to evolve throughout the $20^{\text {th }}$ and $21^{\text {st }}$ centuries in the realm of optical and telecomm wavelengths. SWGs were first implemented in an SOI platform in 2010 [11] as a new form of lossless waveguide, opening up a new field of refractive index engineering and related applications. SWGs are an example of a metamaterial, a structure whose optical properties are determined by its material makeup as well as by its geometric shape. In the case of SOI SWGs, they can have their shape tuned to produce specific refractive indices. They have a wide variety of applications including: waveguides, filters, tapers, edge couplers, photonic bends, waveguide crossings, and resonators. Although most often employed in single-mode devices, recent research has shown great promise in the area of multimode systems. 
a)

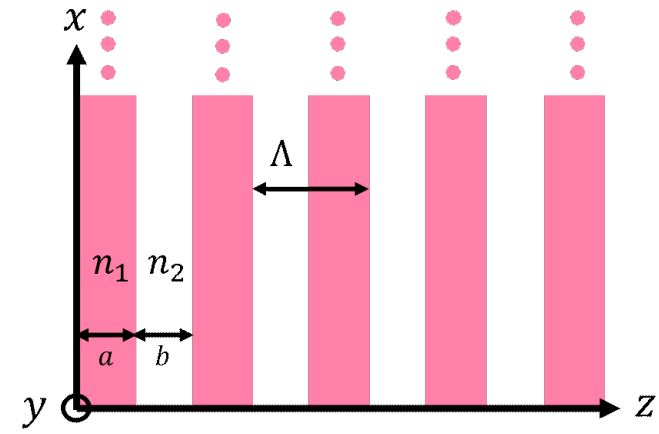

b)

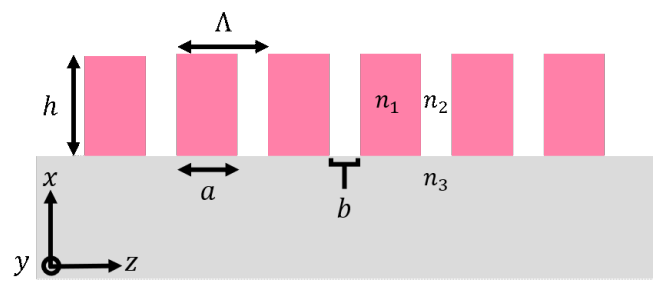

Figure 2.1: a) A one-dimensional subwavelength grating structure, extending infinitely in the $x$ and $y$ dimensions. b) A typical SOI subwavelength grating, finite in $x$ and $y$ and with separate under and upper cladding materials.

\subsubsection{Effective Medium Theory}

The term refractive index is used frequently in the field of photonics, and a distinction should be made between three related, but nonetheless different terms: i) Material index ( $n$ ), ii) Effective index $\left(n_{e f f}\right)$, and iii) Equivalent index $\left(n_{e q}\right)$. Material index is a unit-less quantity that is equal to the ratio of the speed of light in a vacuum to the speed of light through a homogeneous material system, and it is dependent on wavelength. Effective index is a term applied to discretized solutions of Maxwell's equations for a given waveguide, and can be solved for using numerical analysis tools. The effective index of a guided mode varies based on the material indices of the core and cladding materials, the cross-sectional geometry, the mode order, and polarization direction. Equivalent index is a term often associated with subwavelength structures by applying effective medium theory.

It has been shown both theoretically and experimentally that SWGs can be modelled as a waveguide of uniform cross-section with a single equivalent refractive index. This effective medium theory posits that so long as the pitch is sufficiently small compared to 
the wavelength, the SWG acts as a homogeneous material whose index is determined by the polarization direction of the light and the duty-cycle $(a / \Lambda)$ of the periodic segments. The effective medium theory can be thought of as weighted-average of the cladding and core refractive indices, with the duty-cycle as the weighting factor. SWGs are inherently birefringent, as described by Rytov's equations (2.1) and (2.2) [15]. $n_{\|}>n_{\perp}$ in all cases except when the duty-cycle is equal to 1 , in which case that is simply a solid waveguide. For most practical purposes the $n_{\|}$direction is most useful, and can be used to design waveguides where waves propagate in the direction of periodicity.

$$
\begin{aligned}
& n_{\|}^{2}=\frac{a}{\Lambda} n_{1}^{2}+\left(1-\frac{a}{\Lambda}\right) n_{2}^{2} \\
& \frac{1}{n_{\perp}^{2}}=\frac{a}{\Lambda} \frac{1}{n_{1}^{2}}+\left(1-\frac{a}{\Lambda}\right) \frac{1}{n_{2}^{2}}
\end{aligned}
$$

Index engineering in SOI was previously quite limited and depended primarily on a few select parameters. Assuming a silicon core and an $\mathrm{SiO}_{2}$ under cladding, the remaining variables for index tailoring are the upper cladding material, core height, and core width. The choice of upper cladding is generally limited to choices of $\mathrm{SiO}_{2}$, air, or polymer, with one specific refractive index. The height of the silicon device layer is generally restricted to one of few select standardized heights $(220 \mathrm{~nm}, 340 \mathrm{~nm}$, etc.) and partial etch heights add to both the complexity and cost of chips. The width remains the most versatile parameter, but large slopes of $n_{\text {eff }}$ vs width combined with fabrication variations can lead to strong variation in device performance. By combing the choice of width and duty-cycle, SWGs can be tailored to have very specific effective indices, while also being fabrication tolerant by reducing the sensitivity of $n_{\text {eff }}$ with respect to width. This is particularly useful when 
designing MDM systems that have many modes and involve the design of several adiabatic taper sections to couple light in and out. Figure 2.2 shows a sample dispersion sweep of an SWG waveguide for a selection of duty-cycles.

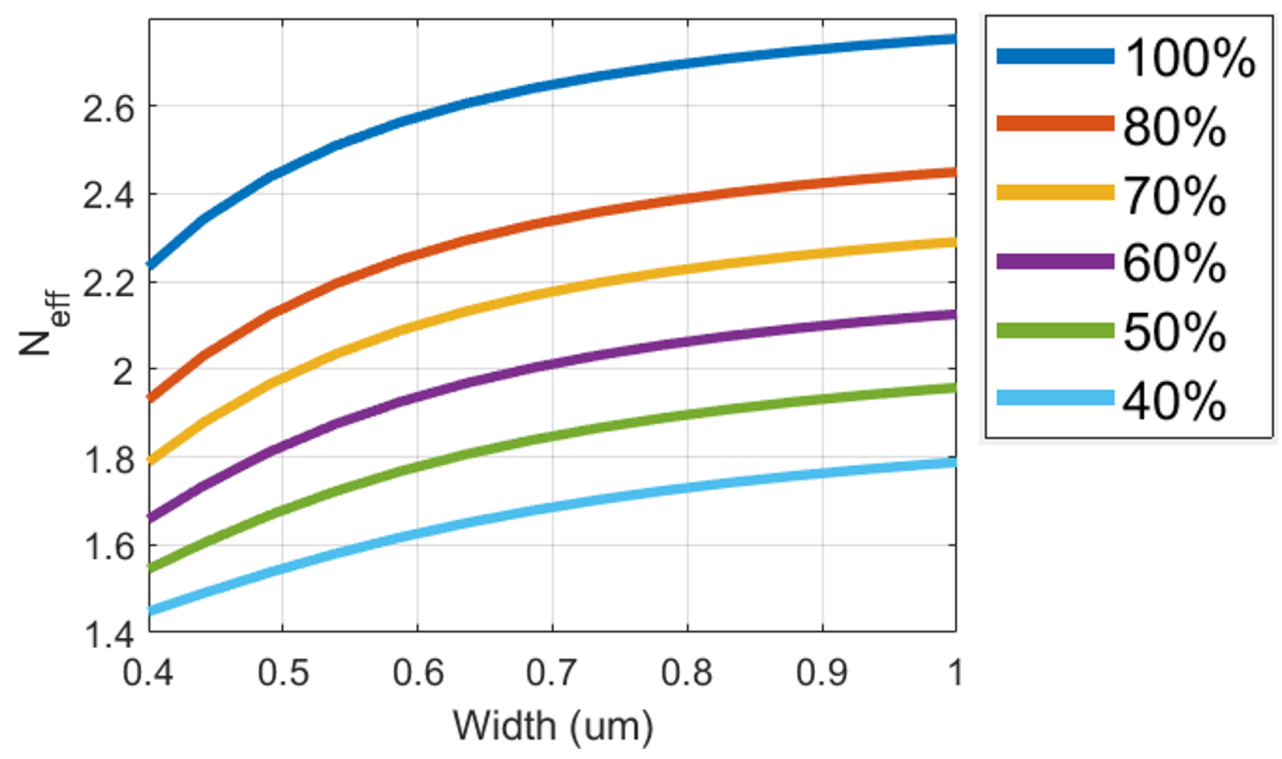

Figure 2.2: Diagram showing the relationship between duty-cycle and effective index for an SWG.

\subsubsection{Band Structures and BlochTheorem}

The effective medium theory is an approximation that only applies to the deep subwavelength regime of $\lambda \gg \Lambda$. It also tends to break down for large SWGs when several modes are propagating at the same time. A full wavelength response for a periodic devices requires a band structure calculation. Bloch theorem has been applied extensively applied in solidstate physics to derive the energy levels of electrons in periodic semiconductor crystals. It can be applied analogously to periodically segmented dielectric media to derive their optical response in terms of wavelength, i.e $\omega$ versus $K$. The electric field vector under Bloch 
theorem is described by equation (2.3), where $K$ is the Bloch wavenumber. Notably it is a periodic function with respect to $\Lambda$.

$$
E=E_{K}(z) e^{-i K z} e^{i\left(\omega t-k_{y} y\right)} \text { where } E_{k}(z)=E_{k}(z+\Lambda)
$$

For periodic media that extend infinitely in all dimensions there exist analytical solutions to the dispersion relation. However for the case of a finite SWG, which confines light in threedimensions, fully-3D numerical analysis tools are required. These numerical treatments will be covered in detail in the relevant sections of chapters 3 and 4 , but for now an overview of an infinite periodic medium will analyzed for demonstrative purposes. For the periodic medium depicted in Fig. 2.3 a), and assuming normal incidence, the dispersion relation is analytically described by equation (2.4). For infinitely large periodic media there is no difference between the TE and TM polarizations under normal incidence, but this is not the case for the finite SWGs which will be discussed later.

$$
\cos (K \Lambda)=\cos \left(k_{1} a\right) \cos \left(k_{2} b\right)-\frac{1}{2}\left(\frac{n_{2}}{n_{1}}+\frac{n_{1}}{n_{2}}\right) \sin \left(k_{1} a\right) \sin \left(k_{2} b\right) \text { where } k_{1,2}=\frac{\omega}{c} n_{1,2}
$$

This equation defines two types of wavelength regions as specified by the following conditions: i) $|\cos (K \Lambda)|<1$, where $K$ is real and the Bloch waves travel freely through the periodic medium, and ii) $|\cos (K \Lambda)|>1$ where $K$ is imaginary and the Bloch waves are evanescent. This corresponds to the photonic band gap which suppresses the propagation of wavelengths in a specific stop-band. This general concept extends to the finite SWGs that confine light in the lateral and vertical dimensions as in Fig. $2.1 \mathrm{~b}$ ), but with small modifications. The three wavelength regions of interest are shown in Fig. 2.3 b). 


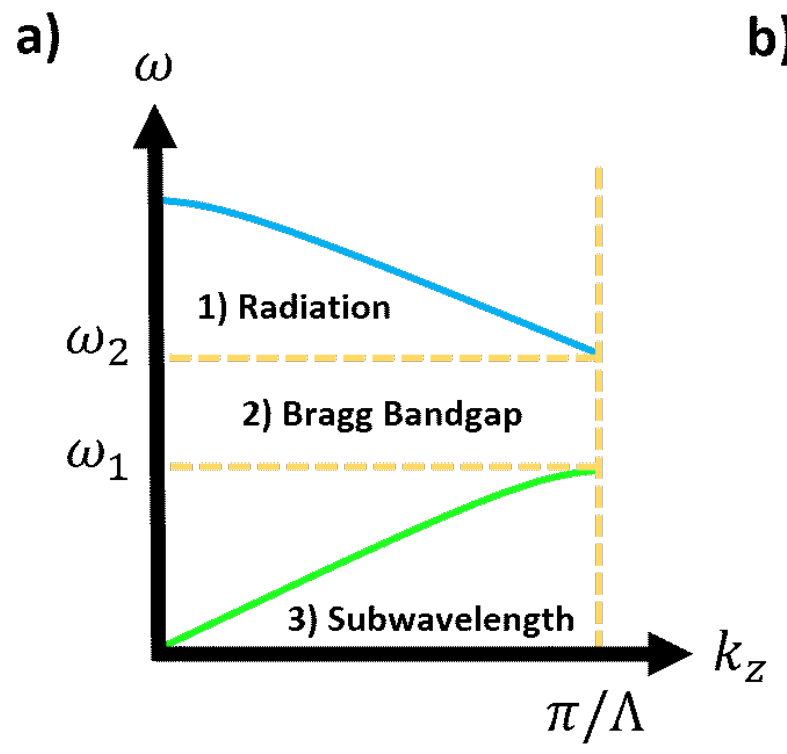

b)

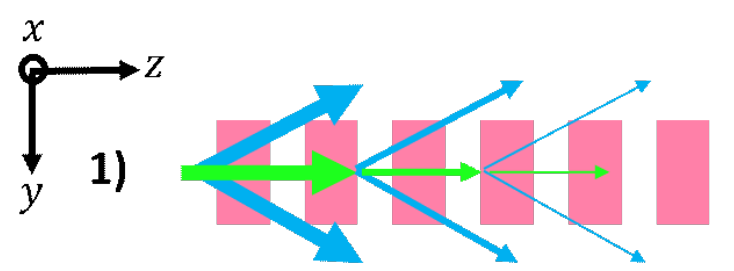

2)

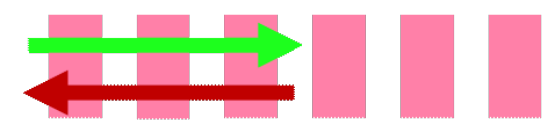

3)

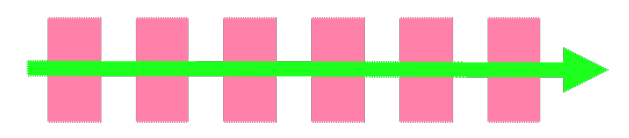

Figure 2.3: a) Optical bandstructure response of a finite periodic structure in $z$. b) Physical response of waves travelling in each wavelength regime.

The first region is the radiative region, for wavelengths comparable in size to the pitch of the grating. In this region light diffracts around the segments, radiation the power away from the core. This effect is useful for designing components such as grating couplers, but for the purposes of this thesis the radiative region is not considered.

The second region occurs at the wavenumber $k=\pi / \Lambda$, and corresponds to a bandgap in the structure, preventing a small range of frequencies from propagating through the structure due to a large number of distributed reflections. This is also known as the Bragg reflection region, and can be used to design highly wavelength selective filters. This will be discussed in more detail in chapter 3 .

The third region is the subwavelength region, where the Bragg reflections are suppressed and the lossless mode propagation occurs. It can seen from the bandstructure that as the $k$ vector approaches zero, the slope $d \omega / d k$ in the subwavelength becomes constant, resulting in 
a nearly constant effective index. The homogeneous effective medium theory arises directly from this result. The modes in this regime differ than that of waveguides of uniform crosssection, and must be modelled instead using Bloch modes, as in Eq. (2.3). Bloch modes are periodic along the axis of the grating, with the field distribution repeating every $\Lambda$. Besides the periodicity, the mode propagates very similarly to how it would in a solid waveguide of the same dimensions, but with a smaller effective index.

\subsection{Simulation Architecture and Tools}

This thesis work primarily used Lumerical's software suite of photonic simulators [16]. This includes Lumerical FDTD for subwavelength gratings and Lumerical MODE which uses finite-difference eigenmode (FDE) calculations for 2D cross-sectional geometries. Lumerical HEAT, which uses the finite-element method (FEM), was employed for thermal simulations of an embedded metal heater. Matlab was employed for a variety of curve calculations, fast Fourier-transforms (FFTs), and data visualization.

\subsubsection{FDTD}

There are many types of electromagnetic simulation architectures. Among the most popular include the finite-element method (FEM), Eigenmode expansion method (EME), and Finite Difference Time Domain (FDTD). Due to the periodic nature of subwavelength gratings they cannot be simulated accurately using FEM or EME, as they assume a uniform cross-section along the entire length of a device. Approximations can be made using the effective-medium theory, but FDTD remains the most accurate solution because it can handle any arbitrary geometries with a sufficiently fine mesh. 
An FDTD simulation applies a rectangular Yee mesh scheme to a 3D geometry as in Fig. 2.4, and propagates an EM field throughout the mesh in discrete time steps. The electric and magnetic fields are updated every alternating half time-step using Maxwell's time domain curl equations. This accuracy comes at the expense of efficiency, as FDTD simulations are often very computationally intensive and require long simulation times that can last anywhere from hours to days. This is due to the fine mesh sizes on the scale of 5-15 nanometers, and fields that must propagate for a time span of thousands of femtoseconds.

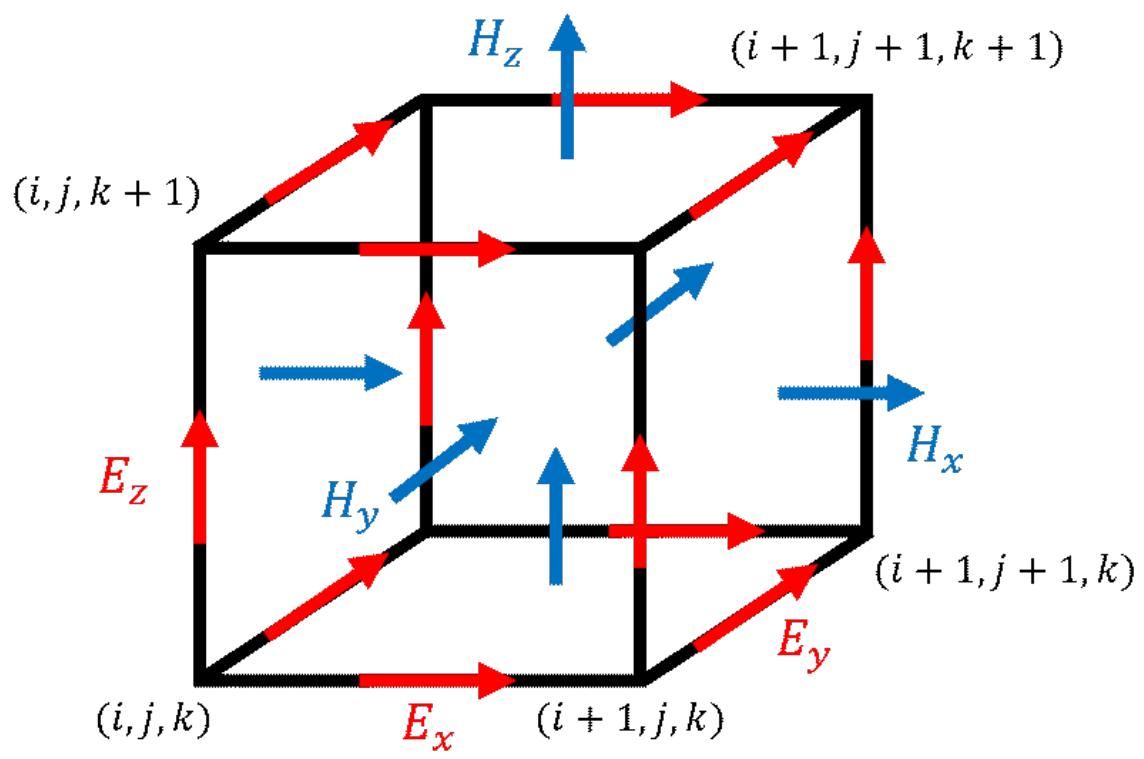

Figure 2.4: Example Yee cell from an FDTD mesh.

SWGs require a two-step process to fully characterize using FDTD. A single unit-cell must be simulated with Bloch-boundary conditions, which treats a single period of the device as if it was repeated infinitely. For a structure that is periodic in $z$, the wavenumber $k_{z}$ is swept from 0 to $\pi / \Lambda_{z}$, where $\Lambda_{z}$ is the period. This corresponds to the first Brillouin zone of the bandstructure, from which the wavelength response of the system can be derived. $k_{z}=\pi / \Lambda_{z}$ 
corresponds to the first bandgap, AKA the Bragg reflection gap, and the frequencies lower than that are those which can propagate in the subwavelength regime. This bandstructure calculation can be used to either find the central Bragg reflection wavelength of a Bragg filter, or the effective index/group index of an SWG.

\subsubsection{FFT}

An FFT is a highly efficient algorithm for computing discrete Fourier Transforms. It is used throughout this thesis work as a means of calculating transmission spectrum data. Wavelength spectrum data can be obtained FDTD simulations with only minimal additional computation time as an FFT is automatically applied to the time-domain data after the simulation has been run. Because the wavelength spectrum data is based on an FFT, simulation time spans must be sufficiently large so as to reduce ripples. Ripples can appears in the output spectra when an FFT is applied to a truncated time span. In the case of the Bragg gratings the simulation time must be on the scale of thousands of femtoseconds in order to propagate throughout the whole device. Light can travel though the multimode bends after a period of 500 femtoseconds, but a longer period upwards of 2000 femtoseconds is ideal. This is because there is a slight resonance effect to the bend, and an accurate FFT of the time data requires the field to have decayed close to zero. FFT simulations were run in Matlab to quickly predict peak splitting behavior in Bragg filters. 


\section{Chapter 3}

\section{Arbitrary Bragg Filter Sidelobe Engineering}

This chapter contains an overview of the design, fabrication, and testing of a set of narrowband Bragg gratings that use a series of silicon loading segments. These Bragg gratings have a weak cosine modulation applied the segments which can tailor arbitrary reflection spectra. These sidelobes may also be thermally induced by supplying an external voltage to a resistive heater. All design, simulation, layout, and analysis are were performed by myself. Fabrication was handled externally by nanophotonic foundry Applied Nanotools, and device measurements were performed by Shurui Wang at the National Research Council facility in Ottawa, Ontario.

\subsection{Bragg Filter Theory}

Bragg gratings are an essential building-block of modern optical systems due to their versatility as a filter. Their applications include wavelength-division multiplexing (WDM), dispersion-compensation and sensing. Although first implemented in optical fibers, Bragg gratings have since been adopted in several material platforms, with the silicon-on-insulator (SOI) platform being of particular interest. The SOI platform leverages the existing complementary metal-oxide semiconductor (CMOS) fabrication infrastructure 
3. Arbitrary Bragg Filter Sidelobe Engineering

to produce dense photonic-integrated circuits at low costs, making it an attractive research avenue for Bragg gratings.

\subsubsection{Bragg Condition and Coupled Mode Theory}

A Bragg grating is a structure with a periodic modulation of its refractive index profile as described in Eq. (3.1). This modulation can be due to a variation in the material properties, or a variation of the geometry of the waveguide, both of which alter the local value of $n_{\text {eff }}$.

$$
n_{e f f}(z)=n_{e f f, 0}+\Delta n(z)
$$

When light propagates along the axis of the modulation a reflection occurs at each indexinterface, and the net result is a large number of distributed reflections. A relatively small wavelength band centered at $\lambda_{B}$ (Eq. (3.2)) interferes constructively and results in a strong reflection, while the majority of the wavelength band passes through the grating unimpeded.

$$
\lambda_{B}=2 n_{e f f} \Lambda_{B}
$$

Coupled mode theory $(\mathrm{CMT})$ is a method of analysis that can be used to solve for the reflectance spectra of a periodic grating structure. It is quite rigorous when considering all modes in a given system, but for weakly coupled grating it is reasonably accurate to discard all but the fundamental forward and backwards propagating modes, simplifying the analysis considerably. The electric field can be expressed as in equation (3.3) as the sum of the forward and backward propagating waves $A(z)$ and $B(z)$.

$$
E(z)=A(z) e^{-j \beta_{0} z}+B(z) e^{j \beta_{0} z} \text { where } \beta_{0}=\frac{2 \pi n_{e f f}(\lambda)}{\lambda_{B}}
$$


$A(z)$ and $B(z)$ satisfy the set of first-order differential equations (3.4). In this context $\kappa$ is the coupling coefficient of the grating, which can thought of as the amount of light coupled to the backward propagating mode per unit distance due to the presence of the grating. It is generally calculated through an overlap integral between the modal field of the unperturbed waveguide and the index perturbation of the grating. $\Delta \beta$ is the phase detuning factor, which is equal to zero at $\lambda_{B}$.

$$
\frac{d}{d z}\left(\begin{array}{l}
A(z) \\
B(z)
\end{array}\right)=-j\left(\begin{array}{cc}
\Delta \beta & \kappa \\
-\kappa & -\Delta \beta
\end{array}\right)\left(\begin{array}{c}
A(z) \\
B(z)
\end{array}\right) \text { where } \Delta \beta=\beta_{0}(\lambda)-\frac{\pi}{\Lambda_{B}}
$$

For a grating of length $L$, the coupled mode equations in (3.4) can be solved by setting the boundary conditions of $A(0) \neq 0$ and $B(L)=0$. The resulting reflectance is shown in equation (3.5).

$$
R=\left|\frac{B(0)}{A(0)}\right|^{2}=\frac{\kappa^{2} \sinh ^{2}(\sigma L)}{\sigma^{2}+\kappa^{2} \sinh ^{2}(\sigma L)} \text { where } \sigma=\sqrt{\kappa^{2}-\Delta \beta^{2}}
$$

\subsubsection{Grating Strength and Bandwidth}

Two key parameters when characterizing a Bragg grating are the grating strength and the bandwidth. Grating strength is directly proportional to the coupling coefficient $\kappa$, which in turn is directly proportional to the strength of the index modulation. For any arbitrary index modulation we can generalize the analysis by taking only the first-order Fourier component as in equation (3.6). The first-order index modulation is then related to the coupling coefficient via equation (3.7).

$$
n(z)=n_{e f f}+\sum_{i} \Delta \frac{n_{i}}{2} \cdot \cos \left(i \cdot 2 \beta_{0} z\right)
$$




$$
\Delta n_{1}=\frac{2 \lambda_{B} \kappa}{\pi}
$$

Control over the grating strength is critical for setting the maximum reflectance and bandwidth of a filter. The maximum reflectance can be solved for by evaluating (3.5) when $\Delta \beta=0$, i.e. $\lambda=\lambda_{B} . R_{\max }$ is described by equation (3.8), which approaches $100 \%$ in the case of an extremely long grating or a grating with a sufficiently strong index modulation, and therefore a strong $\kappa$.

$$
R_{\max }=\tanh ^{2}(\kappa L)
$$

The bandwidth of a grating is defined by the first null-to-null distance in the wavelength band, defined by the condition $\sigma= \pm i \pi$ in equation (3.5). For small bandwidth an analytic expression is given by Eq. (3.9), where $n_{g}$ is the group index at the central Bragg wavelength (Eq. 3.10).

$$
\begin{gathered}
\Delta \lambda=\frac{\lambda_{B}^{2}}{n_{g} L} \sqrt{1+\left(\frac{\kappa L}{\pi}\right)^{2}} \stackrel{\kappa L \gg \pi}{\longrightarrow} \Delta \lambda=\frac{\lambda_{B}^{2} \kappa}{n_{g} \pi} \\
n_{g}=n_{e f f}-\lambda \frac{d n_{e f f}}{d \lambda}
\end{gathered}
$$

For a sufficiently long grating the variable $L$ drops out of equation (3.9), and the coupling coefficient can be extracted from the new equation (3.11).

$$
\kappa=\frac{n_{g} \pi \Delta \lambda}{\lambda_{B}^{2}}
$$


3. Arbitrary Bragg Filter Sidelobe Engineering

\subsection{Literature Review of Bragg Filters in SOI}

Bragg gratings were first implemented in optical fibers by inducing a periodic index variation along the length of a fiber. This was generally achieved by using a mask to expose certain sections to UV light, with the absorption leading to small permanent changes in the refractive index. A similar form of material doping can be performed in SOI platforms where impurities are implanted to change the carrier concentration, thus lowering the refractive index $[17,18]$. However, this technique is limited by the minimum feature size of the impurity doping, which is much larger than that of the grayscale or electron-beam lithography typically used to shape the silicon structures. Custom fabrication processes have been developed to improve the resolution of impurity doping for the purpose of making gratings, but the financial cost has proven to be prohibitive for most applications. The much more common technique for implementing SOI Bragg filters involves a periodic geometry variation of the silicon device layer, which was first demonstrated in 2001 [19].

Bragg gratings in SOI have been traditionally used for creating strong gratings with a large bandwidth. SOI-based Bragg filters often have a strong index modulation because of the high index contrast of the medium $\left(n_{S i}=3.48, n_{S i O 2}=1.44\right)$. Implementing a narrowband Bragg filter requires a weak periodic modulation, which is only achievable with very specific design geometries.

\subsubsection{Waveguide Geometry Perturbation}

There are three primary types of Bragg grating implemented in SOI that use geometry modulation, and they are pictured in Fig. 3.1. 


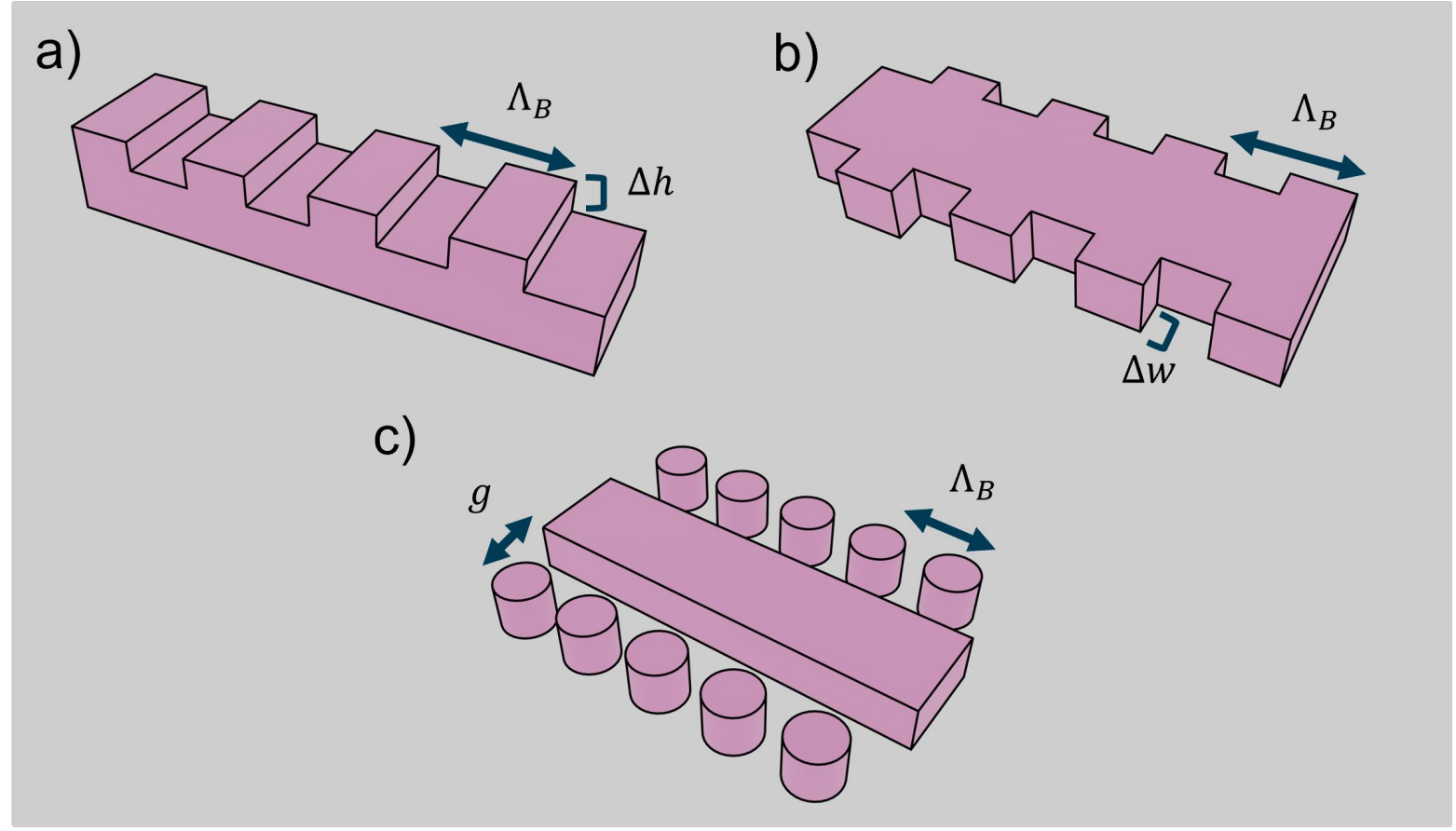

Figure 3.1: a) Height corrugation, b) sidewall corrugation, c) cladding modulation.

\section{Height Corrugation}

A Bragg grating can be implemented vertically by first fully-etching a waveguide, and then using an additional partial etch to corrugate the height of the waveguide. This category of geometry perturbation is uncommon, as it requires an additional etch step whose resolution is limited to approximately $70 \mathrm{~nm}$. The height of the silicon layer tends to vary significantly across large wafer areas, and the large lengths often required for Bragg gratings makes this choice of grating impractical due to fabrication variation [19].

\section{Sidewall Corrugation}

Sidewall corrugation is the most common method of implementing an SOI Bragg filter, as the index perturbation can be implemented in the same etch step as the waveguide core. 
The abrupt change in width changes the effective index of the waveguide with reasonable precision. This perturbation scheme can be implemented with rib-waveguide cores as well, providing an additional degree of design freedom [20]. Limitations on this type of geometry arise due to fabrication sensitivity. The high mode confinement of the SOI platform leads to a large field concentration at the waveguide core's sidewalls, and any fabrication jitter leads to a strong distortion of the output spectra. Grayscale lithography often leads to square shapes becoming rounded, making it difficult to directly transfer designs to functioning circuits. E-beam lithography is able to reach a minimum feature size of $60 \mathrm{~nm}$, but its etching process scales with layout area, increasing both processing time and cost. To achieve bandwidths of $1 \mathrm{~nm}$ and lower requires a sidewall corrugation of around $10 \mathrm{~nm}$, which is not economically feasible with current fabrication infrastructure [21].

\section{Cladding-Modulation}

Cladding-modulated gratings (sometimes referred to as pillar gratings) implement a standard waveguide core with a series of small silicon loading segments placed a certain distance from the core. Cladding perturbation schemes are similar to sidewall corrugation, with the key distinction being the separation of the core and perturbation segments. Compared to sidewall corrugation, cladding-modulated schemes have extremely weak coupling, limiting their usefulness. However, this limitation can be overcome by utilizing an SWG core. First implemented in [22], an silicon wire core was modulated with circular pillars of diameter 200 nm placed a certain gap $(g)$ away from the waveguide. The pillars were small enough to suppress any radiative modes, but large enough to induce the necessary Bragg reflections. The evanescent tail of the waveguide field, and in turn the coupling strength of the grating, exhibited an exponential dependence with respect to $g$. The filter in this work demonstrated 
a narrowband resolution limit of $5 \mathrm{~nm}$ due to the high mode confinement of the wire core, with values of $g$ greater than $300 \mathrm{~nm}$ having a negligible effect on the coupling coefficient.

These results were improved upon in [23], where the loading segments were implemented as squares of side-length $130 \mathrm{~nm}$. By replacing the solid waveguide core with a subwavelength grating (SWG) waveguide and injecting the weakly confined TM polarization, bandwidths as low as $150 \mathrm{pm}$ were demonstrated with gaps as large as $1000 \mathrm{~nm}$. SWG waveguides have a much more loosely confined mode profile, leading to an evanescent field tail extending much further than normal. The Bloch mode profile has a much more gradual exponential field decay, reducing the sensitivity of the coupling strength with respect to the loading segment distance. This allows for a weakly modulated grating that is often required for narrow bandwidths, which can be directly tailored by the pillar distance. Pillar distance is much less limited by the minimum feature size when compared to sidewall corrugation, and is thus far more versatile when designing an arbitrary grating strength.

\subsubsection{Arbitrary Sidelobe Engineering}

Coupled-mode theory predicts that every spatial Fourier component of the index modulation contributes a peak to the reflection spectrum. For an arbitrary transmission spectrum inverse Fourier analysis can be used to obtain the required index modulation as in equation (3.12), where $c_{0}$ is a constant associated with the strength of the grating [24].

$$
n(x)=n_{e f f, 0}+\Delta n(x)=n_{e f f, 0}+c_{0} \mathcal{F}^{-1}[T(\lambda)]
$$

From this result it can be inferred that the superposition of multiple periodicities can be used to arbitrarily design any multi-peak Bragg spectrum. For a given spatial Fourier component of a grating $\Lambda_{i}$ the resulting inter-peak spacing in the transmission spectrum is 
given by equation (3.13). In this case, $\lambda_{B}$ is the central wavelength of the unmodulated grating and $n_{g}$ the group index at $\lambda_{B}$.

$$
\Delta \lambda_{i}=\frac{\lambda_{B}^{2}}{2 n_{g} \Lambda_{i}}
$$

\section{Multiple Periodicities}

Multi-period geometries are possible through a variety of solutions, the most straightforward of which is a cascaded Bragg grating. Cascaded Bragg gratings are a set of single-period Bragg gratings connected in sequence. Although easy to implement, the total device footprint is quite large, as the total device length is multiplied by the total number of cascaded gratings. Because of this fact they are also susceptible to variations in the silicon thickness across a large wafer area, and thus may be difficult to translate from simulation to fabricated design.

Asymmetric sidewall corrugation can be utilized to inscribe two distinct periodicities on a silicon waveguide [25]. Each sidewall of the waveguide has a unique period as well as a unique corrugation width to tailor the coupling strengths. The multi-period grating may be treated as the superposition of two separate gratings, each with only one sidewall corrugation. This approach successfully implements two central Bragg reflection peaks, but the potential coupling strength of each grating is halved compared to a design that utilizes both sidewalls. Rib waveguides have demonstrated two-period Bragg gratings by separately modulating the slab and rib sections [26]. This design can even be extended to a four-period grating through the previously discussed asymmetric sidewall corrugation, with two gratings on the slab level and two gratings on the rib level.

Sampled gratings have been shown as an effective way to implement a uniformly spaced frequency spectrum comb [21]. A sampled grating can be layered on top of a standard 
3. Arbitrary Bragg Filter Sidelobe Engineering

grating by removing the sidewall corrugations at a set period. The coupling strength and comb-spacing of the spectrum are determined entirely by the duty-cycle and period of the sampling function, respectively.

Previous work [24] has successfully converted an arbitrary transmission spectrum into the required SOI sidewall corrugation. First a desired transmission spectrum is chosen, and then by taking the inverse Fourier transform of the output spectrum the required index modulation is calculated as in equation (3.12). From this point it is a matter of finding the relationship between effective index and device width, and calculating the required width modulation. Finally, the width modulation is truncated to follow the minimum feature size of the chosen fab-process. However because the filter studied used sidewall corrugation, the control over the effective index was constrained by minimum feature size. A claddingmodulated scheme that controls the effective index via the pillar gap allows for much more precision, and is a far superior application of this inverse Fourier-design process.

\section{Electro-Optic and Thermal Modulation}

SOI Bragg gratings can be combined with active components controlled by external voltages, either through carrier injection or the thermo-optic effect. A fully-reconfigurable Bragg grating for photonic signal-processing has been demonstrated using a carrier-injection modulated Fabry-Perot cavity [27]. On-chip dispersion control has been demonstrated by implementing a tunable temperature gradient over-top a Bragg grating [28], with a linearly tilted heater chirping the grating. Tunable thermal superstructures were first demonstrated in optical fibers in [29], where periodically heated Bragg gratings demonstrated a variety of tunable filters. More recently a similar super-structure was demonstrated in SOI using five equidistant heating elements over-top a 
Bragg grating, which allowed the band-pass filter to be dynamically switched to a frequency-comb [30].

\subsection{Proposed Design}

Bragg filters with narrow bandwidths in the range of $8 \mathrm{~nm}$ to $150 \mathrm{pm}$ were previously implemented in [23]. The minimum bandwidth limit has been decreased thanks to the low mode confinement of the SWG core, allowing the loading segment gap to be much larger than before. As pictured in Fig. 3.2 a), loading segments placed a distance $g$ from the SWG core and with an offset of $\xi$ allowed for precise control of the strength of the grating. The loosely confined mode profile of the SWG resulted in an evanescent tail that extended far out to the Bragg loading segments. This geometry allows for a weak Bragg grating in spite of the high index contrast of the SOI platform.

For gratings with lengths in the millimeter range and values of $g$ larger than $500 \mathrm{~nm}$, bandwidths in the sub-nanometer range are possible, as pictured in Fig. $3.2 \mathrm{~b}$ ). This is a remarkable result for a device with feature sizes greater than $100 \mathrm{~nm}$. The previous work [23] demonstrated that the best performing devices used the following geometry: An SWG of $h$ $=220 \mathrm{~nm}, w=450 \mathrm{~nm}$, duty-cycle $=60 \%, \Lambda_{S W G}=242 \mathrm{~nm}$, and $\Lambda_{B}=484 \mathrm{~nm}$. The best narrowband filter results used the TM polarization and had $g=1000 \mathrm{~nm}$ and $\xi=200 \mathrm{~nm}$. This design can be built upon to design arbitrary transmission spectra with precise control and without the need for inverse design algorithms. 
a)

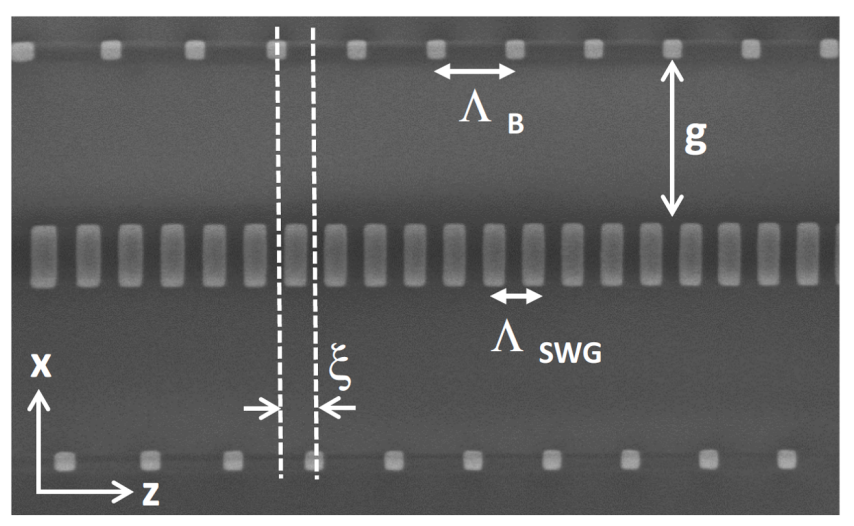

b)

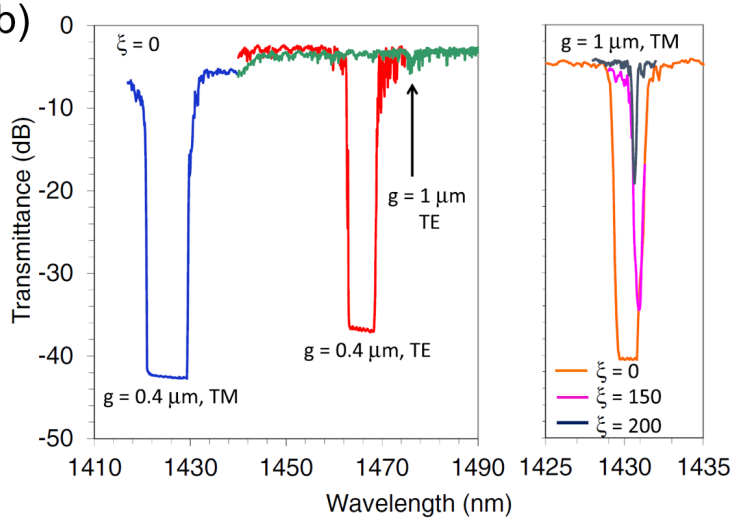

Figure 3.2: a) Scanning electron microscope image of cladding-modulated Bragg gratings with an SWG core. b) Measured transmission spectra of the fabricated filters. Reprinted with permission from [23] () The Optical Society.

By introducing a cosine envelope to the gap of the Bragg grating, sidelobes can be introduced to the output spectrum. By tuning the amplitude of the cosine wave, the period, and the offset between both sides of the filter, all the parameters of the transmission spectrum can be set. This includes the extinction ratio, the bandwidth, and the peak spacing. By adding multiple cosines to the modulation envelope many different output spectra can be layered on top of each other as a direct result of Fourier analysis. This allows for theoretically unlimited scaling of periodicities and the creation of arbitrary transmission spectra. The general equation describing an arbitrary cosine modulation is seen in equation (3.14), and is visualized in Fig. 3.3.

$$
g(z)=g_{0}+g_{i} \cos \left(\frac{2 \pi z}{\Lambda_{i}}\right)+\ldots
$$




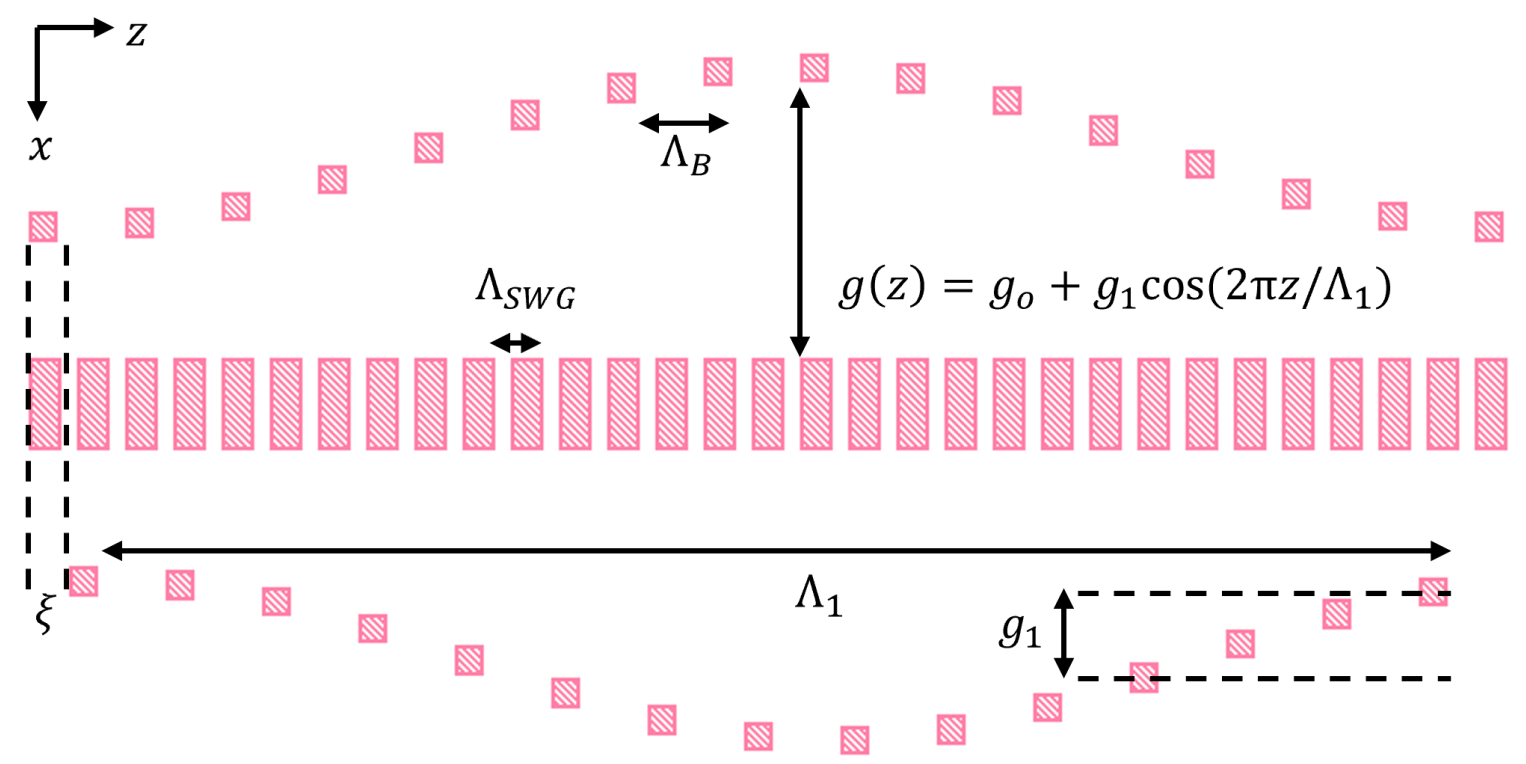

Figure 3.3: Layout demonstrating a single cosine modulation of an SWG Bragg grating.

\subsection{Bragg Grating Band Structure}

As discussed previously, the central Bragg wavelength of a grating is given by $\lambda_{B}=2 n_{e f f} \Lambda_{B}$. $n_{\text {eff }}$ refers to that of the unperturbed waveguide core, but it will be shown in the following subsections that the size of $g$ has a non-negligible impact on $\lambda_{B}$. Although the focus of this thesis work is the fundamental TM mode, the fundamental TE mode will also be discussed in this section to help demonstrate the underlying physical mechanisms. The waveguides have a cross-sectional geometry of $h=220 \mathrm{~nm}, w=450 \mathrm{~nm}$, and a Bragg period of $\Lambda_{B}=$ $484 \mathrm{~nm}$. Furthermore, the SWGs have $\Lambda_{S W G}=242 \mathrm{~nm}$ and a duty-cycle of $60 \%$. 
3. Arbitrary Bragg Filter Sidelobe Engineering

\subsubsection{Unperturbed Waveguide Characterization}

There are four cases to consider in this section: SWG-core TE, SWG-core TM, wire-core TE, and wire-core TM. The TE modes for both core-types will have larger effective indices and strong modal field confinement, reducing how far their evanescent field tail extends. This renders them less useful for the purposes of designing weak Bragg gratings, as they are more sensitive to fabrication jitter and insensitive to $g$. The SWG-cores will have much lower effective indices, and modal fields that extend much further from the waveguide core. This increases the useful range of $g$ values for the SWG-cores when compared to the wire-cores, but with the potential downside of a lossier mode. To fully characterize the Bragg response it is necessary to obtain the wavelength response of $n_{e f f}$ and $n_{g}$ for each of the four cases.

To characterize the SWG cores Lumerical FDTD includes a bandstructure analysis group which consists of a set of randomly distributed time-domain field monitors. The normalized Bloch wavenumber $K$ is swept from 0 to 0.5 and the results from the time-domain monitors have an FFT applied to them. The frequency-domain response is then plotted on a logarithmic scale, as pictured in Fig. 3.4. In this case the red frequencies represent the guided SWG modes, whereas the light blue lines represent the light-line of the $\mathrm{SiO}_{2}$ cladding. The values of $n_{\text {eff }}$ and $n_{g}$ can be extracted by calculating the phase and group velocities, as seen in Eq. (3.15) and (3.16) (note that $\beta=2 \pi k / \Lambda$ and $\omega=2 \pi f$ ).

$$
\begin{aligned}
& n_{\text {eff }}=\frac{c}{v_{\text {phase }}} \text { where } v_{\text {phase }}=\frac{\omega}{\beta} \\
& n_{g}=\frac{c}{v_{\text {group }}} \text { where } v_{\text {group }}=\frac{d \omega}{d \beta}
\end{aligned}
$$

The wire-core was analyzed in Lumerical MODE using the finite-difference eigenmode 
a)

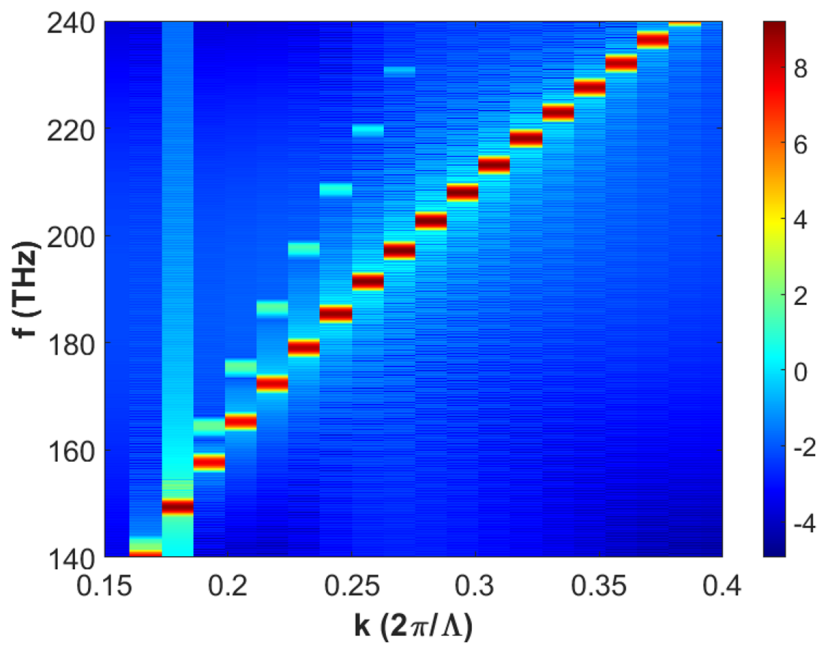

b)

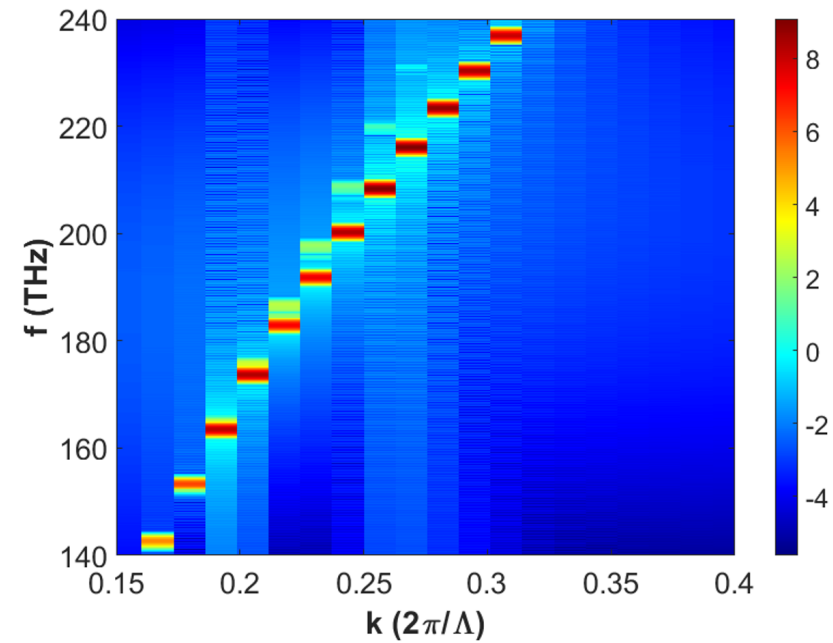

Figure 3.4: a) $\mathrm{TE}_{0}$ mode bandstructure response. b) $\mathrm{TM}_{0}$ mode bandstructure response.

simulation scheme. The values of $n_{e f f}$ and $n_{g}$ can be obtained directly from the simulation data. The values of $n_{\text {eff }}$ and $n_{g}$ versus wavelength are plotted for all four cases in Fig. 3.5 a) and b), respectively.

a)

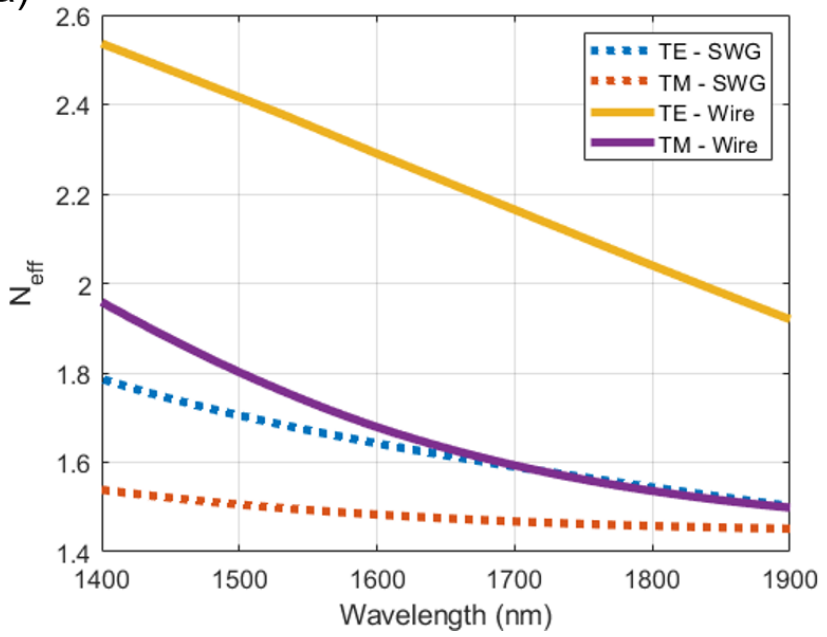

b)

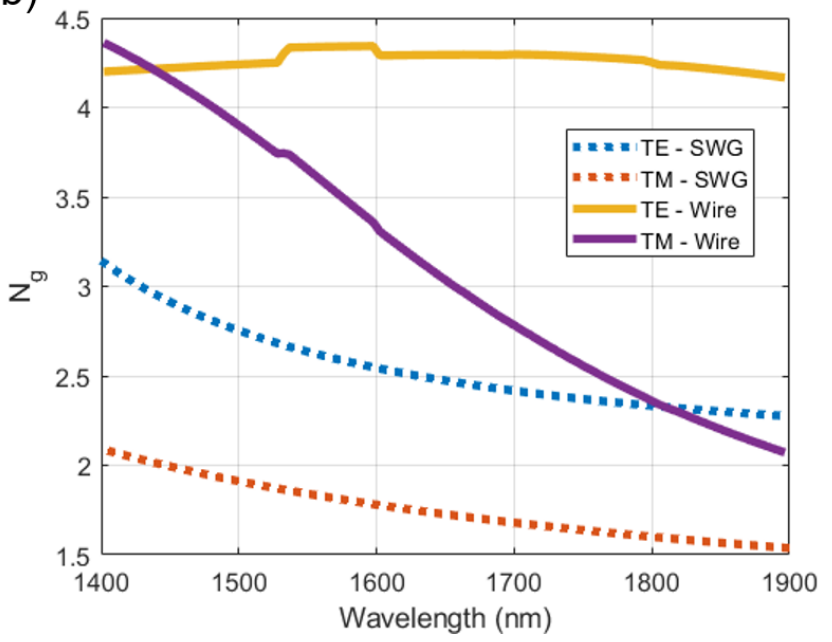

Figure 3.5: a) $n_{\text {eff }}$ versus wavelength. b) $n_{g}$ versus wavelength.

It is clear from the Bragg condition equation that $\lambda_{B}$ is directly proportional to $n_{e f f}$. 
The exact value of $n_{\text {eff }}$ (and by extension $\lambda_{B}$ ) depend on factors such as $g$ and $\xi$, but for wavelengths below $1650 \mathrm{~nm}$ the following condition will always hold true: $\lambda_{T M, \mathrm{SWG}}<$ $\lambda_{T E, \mathrm{SWG}}<\lambda_{T M, \text { Wire }}<\lambda_{T E, \text { Wire }}$.

\subsubsection{Relationship between Gap \& Effective Index}

In order to predict the spectral response of the modulated gratings a relationship between $g$ and $\kappa$ had to be established. Lumerical 3D FDTD was used to simulate a single unitcell of a Bragg grating with Bloch-Boundary conditions, effectively simulating the spectral response of an infinitely long grating. Much like for the SWG bandstructure analysis in section 3.4.1 the wavelength response can be extracted, but due to the presence of the Bragg loading segments a photonic bandgap is produced. This bandgap allows the central Bragg wavelength and bandwidth to be extracted. Assuming the case of an infinitely long grating it is also possible to extract the coupling coefficient $\kappa$ from Eq. (3.11) by using the values of $n_{g}$ calculated in section 3.4.1.

This bandstructure analysis was run for a sweep of $g$ values, for both the wire-core (Fig. 3.6) and SWG-core geometries (Fig. 3.7), as well as for both polarizations. In all four cases, whether TE or TM, wire-core or SWG-core, the coupling coefficient due to the Bragg segments follows an inverse logarithmic curve with respect to $g$. This is a direct result of the exponential decay of the evanescent field from the core. An exponential curve fit was applied to the coupling coefficient

For the wire and SWG variants three gap sizes were chosen for further investigation. These values and their corresponding $\lambda_{B}$, bandwidth, $n_{\text {eff }}$ and $n_{g}$ values are listed in table 3.1. 
a)

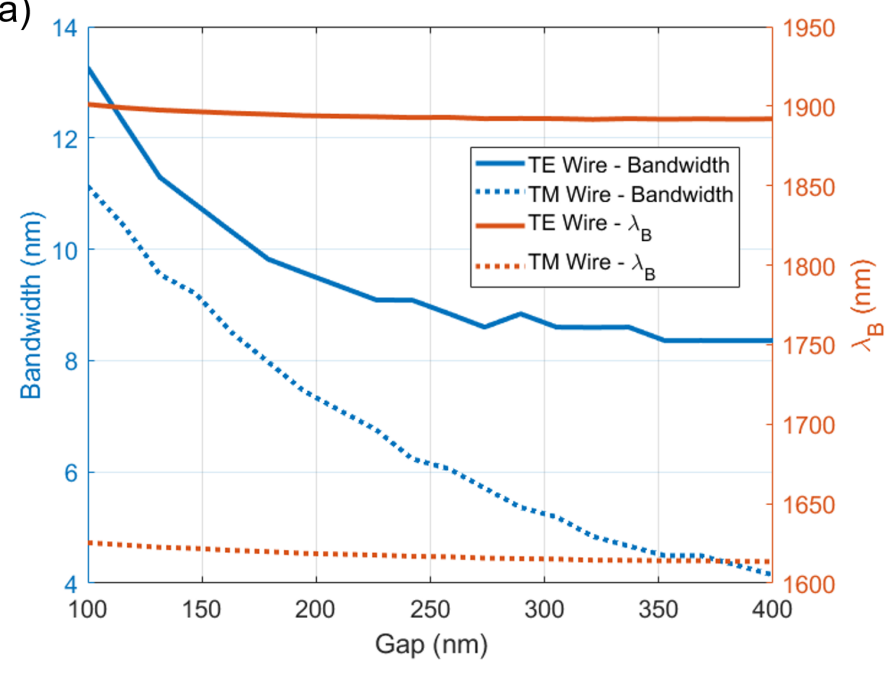

b)

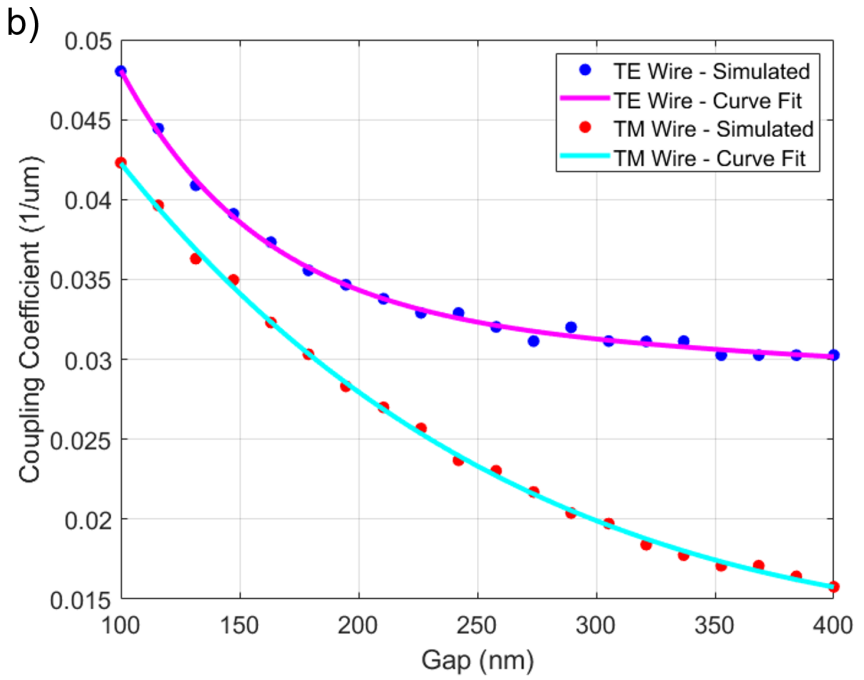

Figure 3.6: a) Dependence of bandwidth and central Bragg wavelength on $g$ for a wire core. b) Calculated values of $\kappa$ and the exponential curves fits.

a)

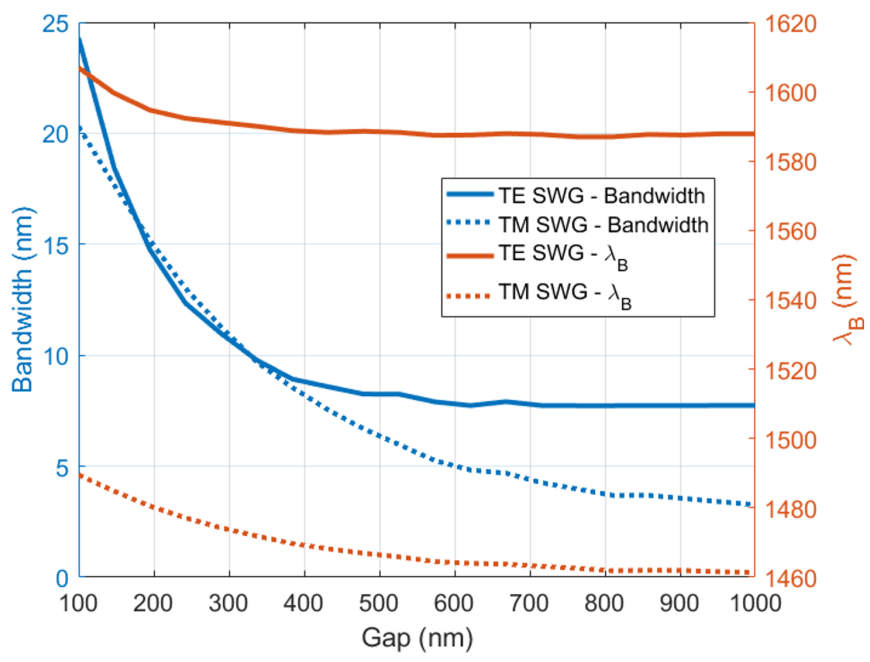

b)

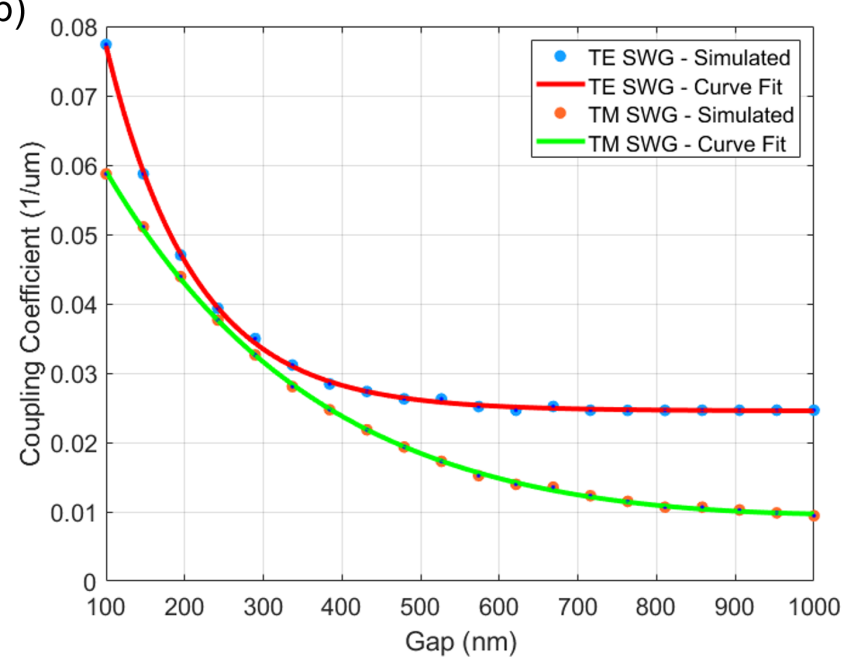

Figure 3.7: a) Dependence of bandwidth and central Bragg wavelength on $g$ for an SWG core. b) Calculated values of $\kappa$ and the exponential curves fits. 
Table 3.1: Summary of the key Bragg parameters for six $g$ values and both TE and TM polarization.

\begin{tabular}{|c|c|c|c|c|c|c|c|c|}
\hline- & \multicolumn{3}{|c|}{ TE } & \multicolumn{3}{c|}{ TM } \\
\hline$g(\mathrm{~nm})$ & $\lambda_{B}(\mathrm{~nm})$ & $\Delta \lambda(\mathrm{nm})$ & $n_{\text {eff }}\left(\lambda_{B}\right)$ & $n_{g}\left(\lambda_{B}\right)$ & $\lambda_{B}(\mathrm{~nm})$ & $\Delta \lambda(\mathrm{nm})$ & $n_{e f f}\left(\lambda_{B}\right)$ & $n_{g}\left(\lambda_{B}\right)$ \\
\hline SWG - 1000 & 1588 & 7.7 & 1.65 & 2.56 & 1461 & 3.3 & 1.52 & 1.97 \\
SWG - 750 & 1588 & 7.8 & 1.65 & 2.56 & 1463 & 4.0 & 1.52 & 1.97 \\
SWG - 500 & 1589 & 8.3 & 1.65 & 2.56 & 1466 & 6.4 & 1.52 & 1.97 \\
Wire - 350 & 1892 & 8.4 & 1.93 & 4.17 & 1614 & 4.5 & 1.66 & 3.24 \\
Wire - 225 & 1893 & 9.1 & 1.93 & 4.17 & 1618 & 6.8 & 1.66 & 3.22 \\
Wire - 180 & 1895 & 9.8 & 1.93 & 4.17 & 1620 & 8.0 & 1.66 & 3.21 \\
\hline
\end{tabular}

\subsection{Geometry Modulation}

For all filter variants discussed in this section two key types of simulations were performed: i) A numerical FFT using curve-fit data from section 3.4.2, and ii) a fully 3D FDTD simulation of the device geometry. The FFT simulations were used to obtain a quick relative estimate of the expected wavelength response by normalizing the central Bragg reflectance peak to $0 \mathrm{~dB}$. It can provide a good estimate of relative sidelobe heights and spacings, but it cannot model peak bandwidth or material dispersion. The FDTD simulations are much more computationally intensive and take much longer to complete, but provide a highly accurate prediction of the wavelength response of a given filter variant. For demonstrative purposes the focus of this section is the SWG-core variant with a TM input, but the analysis could easily be extended to either wire-core or TE inputs. 


\subsubsection{Numerical FFT Simulations}

As per coupled-mode-theory, every spatial frequency component of an index modulation contributes a peak to the wavelength spectrum of a Bragg grating. By associating the $g(z)$ value with the grating's coupling coefficient $\kappa(z)$, the index modulation $\Delta n(z)$ can be calculated using equation (3.7). By performing a numerical FFT on $\Delta n(z)$ it can generate a power density spectrum that separates the various frequency components of the index modulation. By centering the peak contribution from $g_{0}$ to $\Lambda_{B}$ and normalizing it to $0 \mathrm{~dB}$, a normalized reflectance spectrum can be generated.

The first step is to define $g(z)$ in terms of the unmodulated gap $g_{0}$, and any additional cosine modulation terms. A sample modulation with a single cosine is shown in Fig. 3.8 a), where $g_{0}=1000 \mathrm{~nm}, g_{1}=400 \mathrm{~nm}$, and $\Lambda_{1}=50 \Lambda_{B}=24.2 \mu \mathrm{m}$. Each black point represents the position of a single Bragg loading segment relative to the core. By using the previously calculated curve fit of $\kappa$ for the TM-SWG core, the modulated value of $\kappa(g(z))$ can be calculated. This result is plotted in Fig. $3.8 \mathrm{~b}$ ), producing a distorted cosine function due to the exponential curve-fit. The equivalent index perturbation due to the modulation is shown on the second axis. 

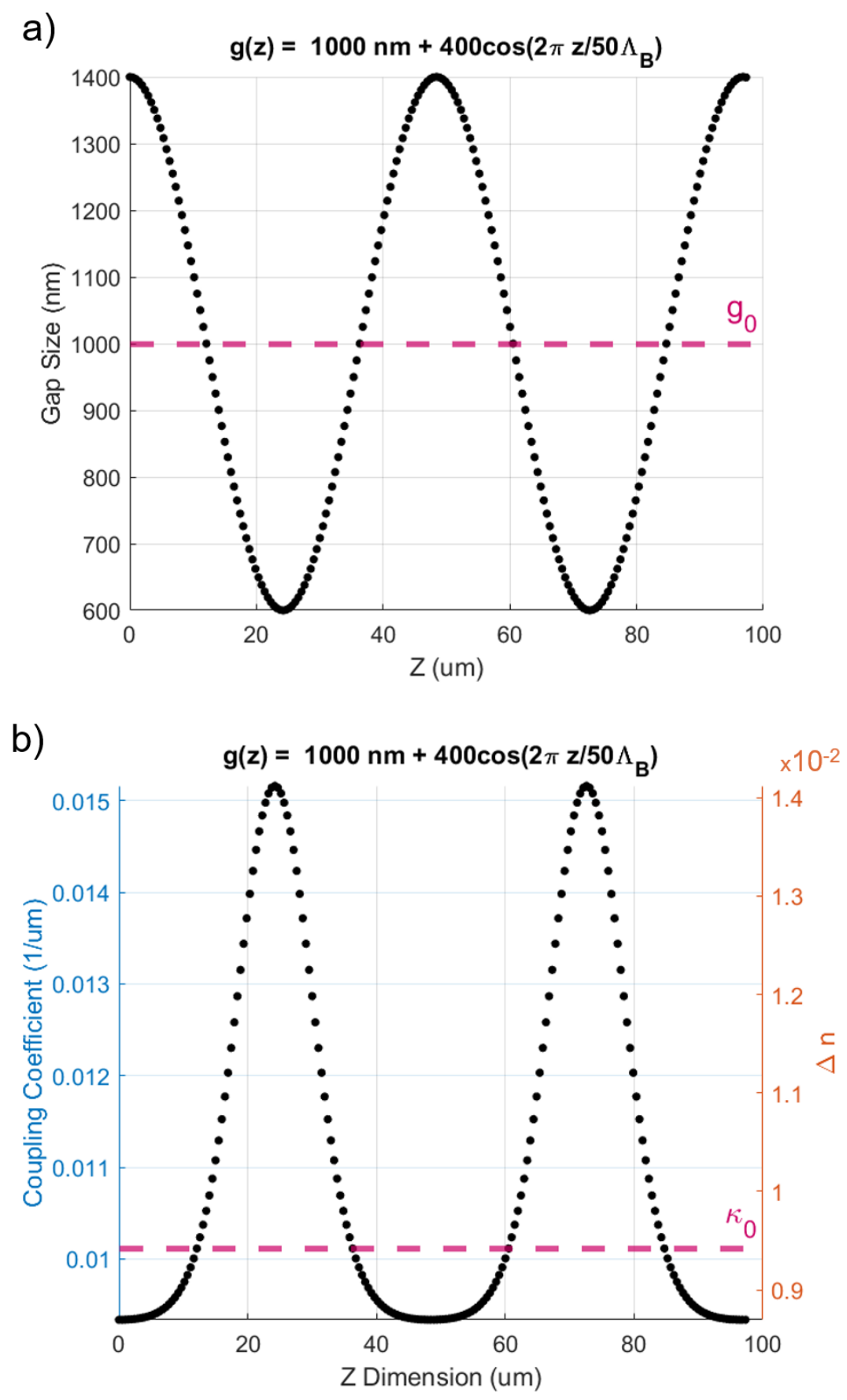

Figure 3.8: A sample modulation with a single cosine: a) Position of the Bragg segments as a function as distance. b) The corresponding coupling coefficient as a function of distance and the equivalent change in index perturbation. 
Four sample numerical FFTs are shown in Fig. 3.9, each with a different single cosine modulation. Graphs a) and b) are modulated with a cosine with a period of $50 \Lambda_{B}$, but with different amplitudes. It can be seen that increasing the modulation from 100 to 400 $\mathrm{nm}$ significantly increases the max reflectance of the sidelobes while maintaining the same interpeak spacing. Similarly, graphs c) and d) show a similar effect, but for a modulation period of $100 \Lambda_{B}$. Doubling the period halves the interpeak spacing, and increasing the amplitude increases the max reflectance of the sidelobes.
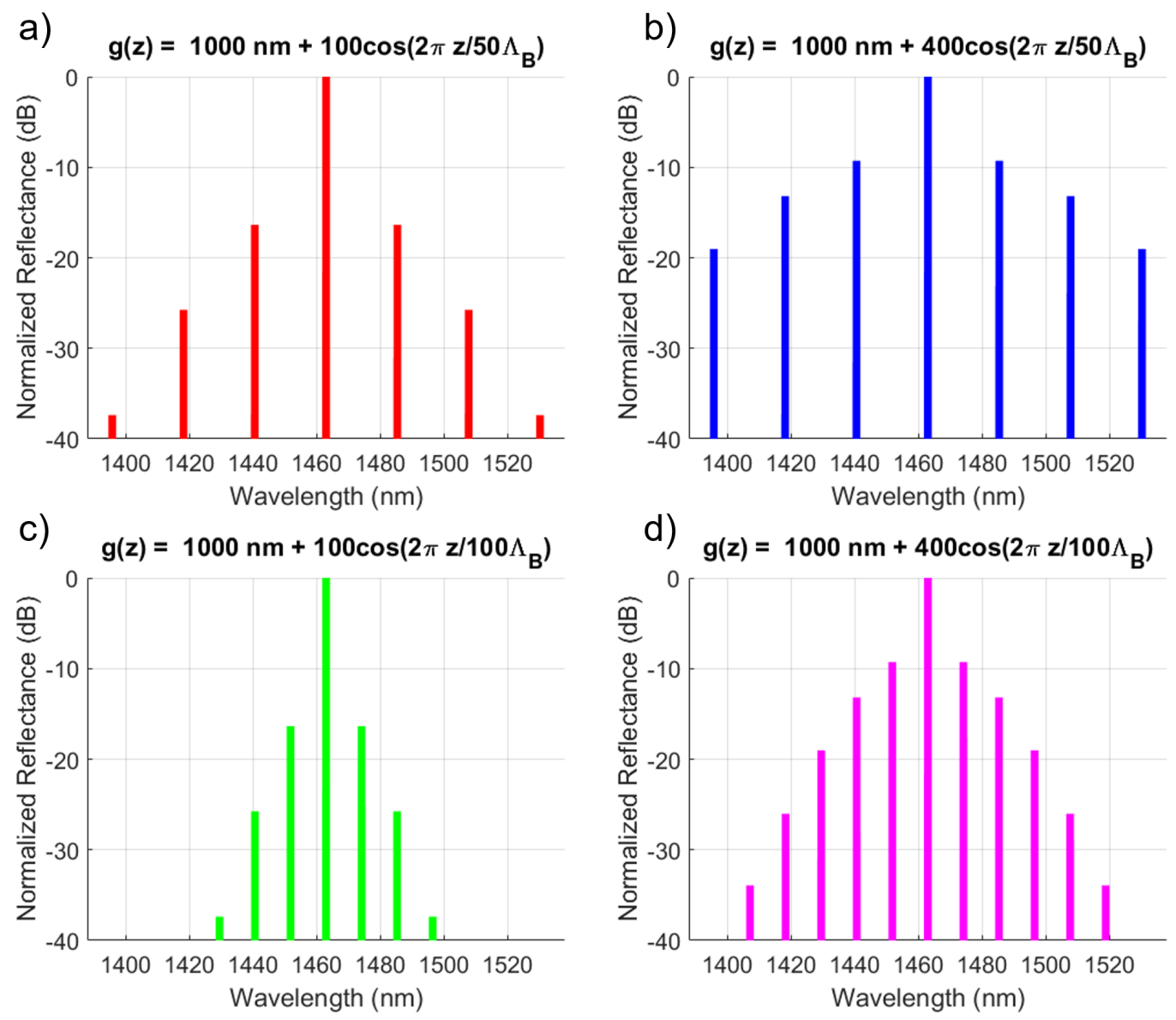

Figure 3.9: Four numerical FFT spectra, each of which has a unique single cosine modulation. 
More complex spectra can be arbitrarily engineered by layering multiple cosines in $g(z)$. Fig. 3.10 showcases two numerical FFT spectra with two cosine modulations each. Because the modulations periods of $100 \Lambda_{B}$ and $25 \Lambda_{B}$ are multiples of each other, the fourth sidelobe from the center is a local maximum, creating a sidelobe envelope. The maximum reflectance of this sidelobe envelope can be further tailored by the ratio of the cosine amplitudes.
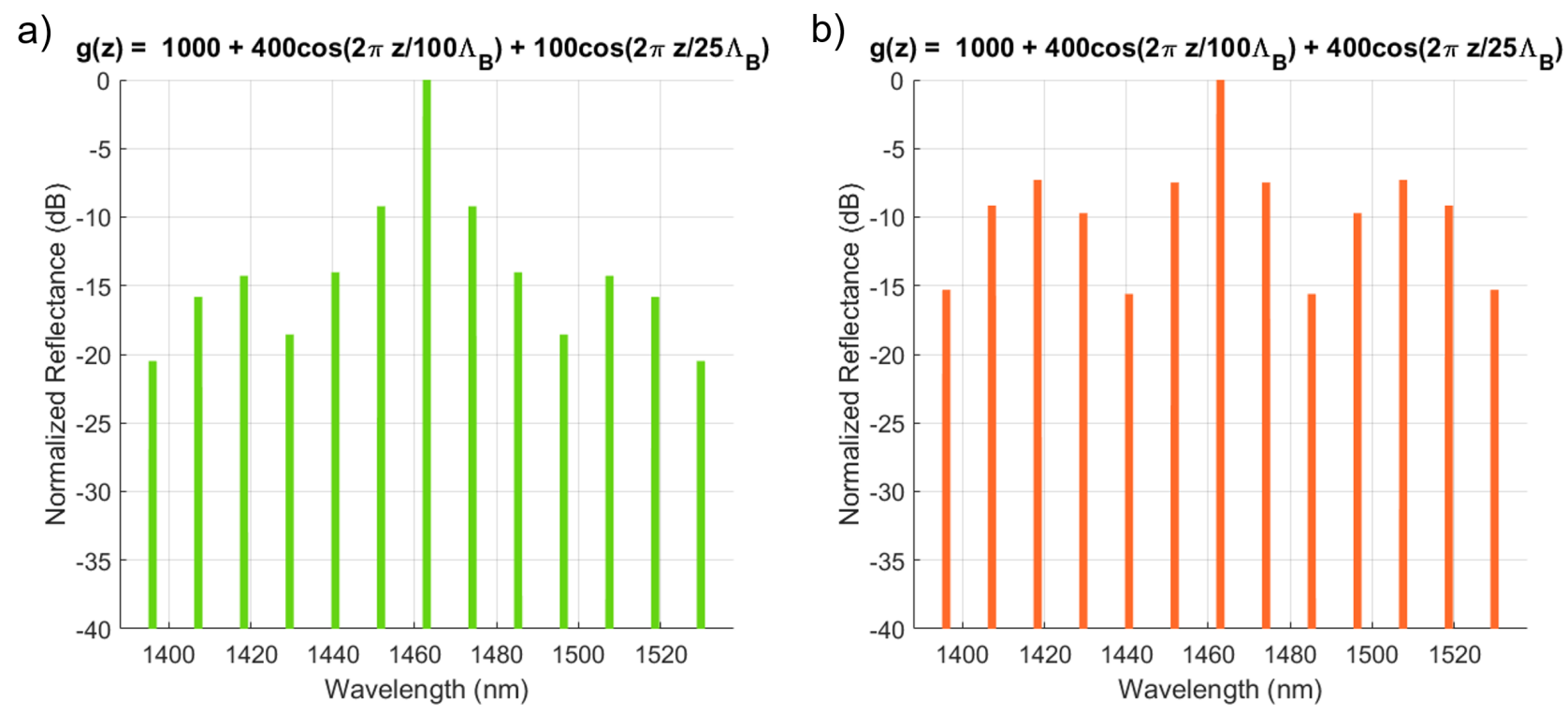

Figure 3.10: Two numerical FFT spectra, each of which has a unique double cosine modulation.

\subsubsection{D FDTD Simulations}

Although computationally expensive, fully 3D FDTD simulations provide the best accuracy when characterizing subwavelength structures. Lumerical FDTD was used to simulate a variety of cosine modulation variants of the TM-SWG filter core. The structures were generated using Lumerical's scripting language, and consist of 1000 Bragg periods. The fundamental TM mode was injected using into a wire-core of cross-section $450 \mathrm{~nm}$ by 
$220 \mathrm{~nm}$ before being slowly tapered into an SWG. The reflectance spectra were obtained from the absolute square of the S-parameter of the injection port. Fig. 3.11 shows the reflectance an unmodulated grating as well as four distinct modulation periods. All five curves overlap at the central Bragg wavelength of $1467 \mathrm{~nm}$. This central wavelength differs from the Bloch-boundary simulation value of $1461 \mathrm{~nm}$ by approximately $6 \mathrm{~nm}$. This discrepancy is likely due to differences in the simulation measurement techniques. Whereas the Bloch-boundary method applies an FFT to the time-domain result of ten randomly distributed point-like field monitors, the full device simulation is measuring the power transfer through a two-dimensional cross-section. Increasing the period results in a smaller inter-peak spacing, in agreement with equation (3.13). Only one pair of sidelobes is visible for each modulation because the amplitude of $100 \mathrm{~nm}$ is relatively weak compared to the $g_{0}$ of $1000 \mathrm{~nm}$. The sidelobes are asymmetric, with those at higher wavelengths possessing a lower maximum reflectance, likely a result of material dispersion.

Fig. 3.12 shows the reflectance of an unmodulated grating as well as two gratings with different amplitudes, but the same period. As expected all three gratings share the same central peak, but the modulated gratings produce visible sidelobes of varying height. The weakly modulated grating produces two visible sidelobes of equal spacing, whereas the strongly modulated grating produces four visible peaks of equal spacing. This suggests the ability to create a comb-like filter using a sufficiently large modulation amplitude.

Previous work has shown that introducing an offset $(\xi)$ between the two rows of Bragg loading segments can further weaken the strength of the grating, reducing the bandwidth of the filter. This is a direct result of the coupling coefficient decaying according to a cosine law function as described in equation (3.17). 


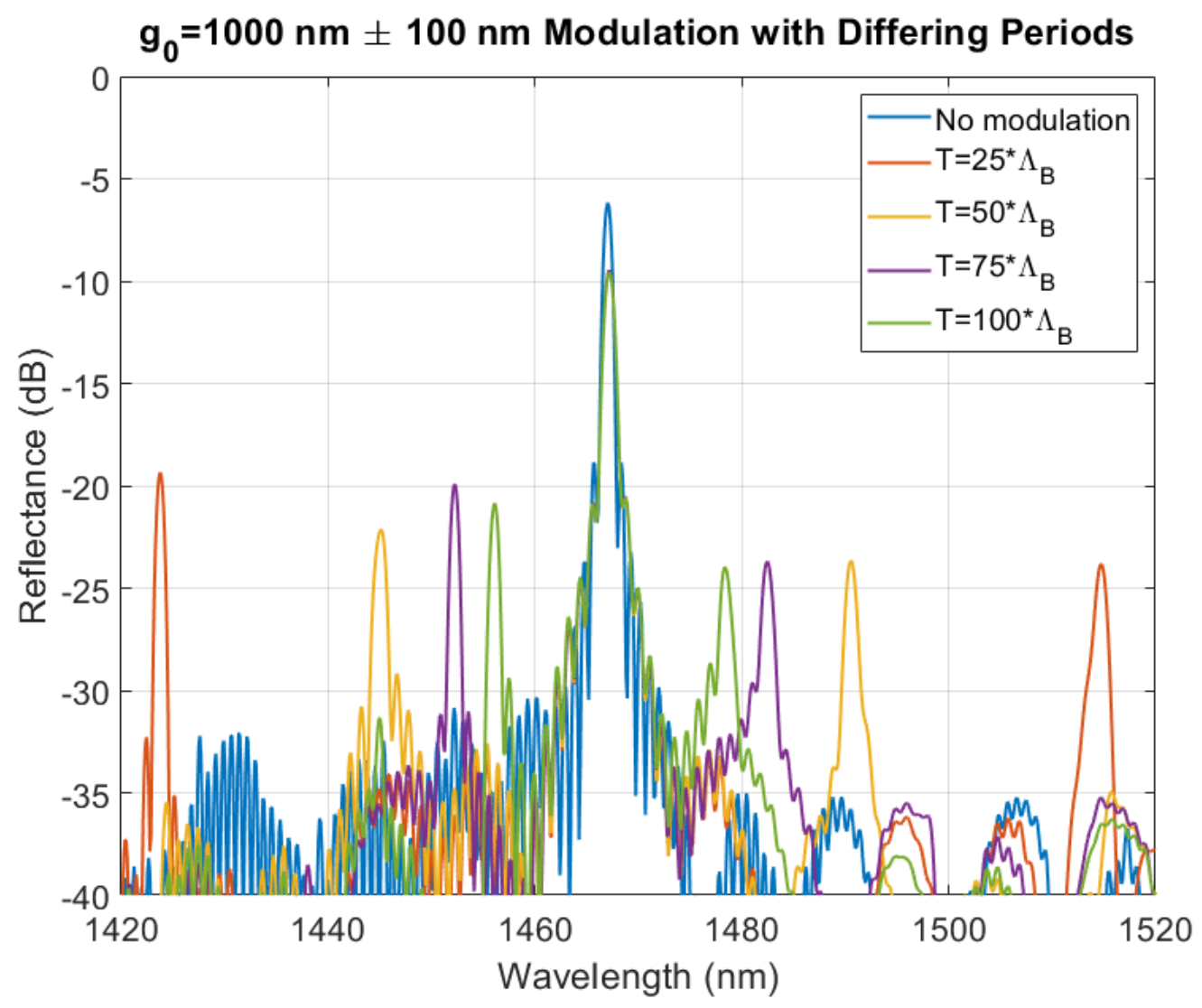

Figure 3.11: Reflectance spectra of an unmodulated TM-SWG grating compared to four modulations with different periods.

$$
\kappa(\xi)=\kappa(0) \cos \left(\frac{\pi \xi}{\Lambda_{B}}\right)
$$

This relationship was first demonstrated for sidewall corrugation in [31] and then experimentally demonstrated for cladding-modulated Bragg gratings in [23], where sub-nanometer bandwidths were achieved. As the Bragg segments have a period of $\Lambda_{B}$, the range of possible values is $0 \leq \xi \leq \Lambda_{B} / 2$. This is shown to have a small impact on $\lambda_{B}$ (less than $1 \mathrm{~nm}$ ), with larger shifts occurring for smaller values of $g$. In addition to narrowing 


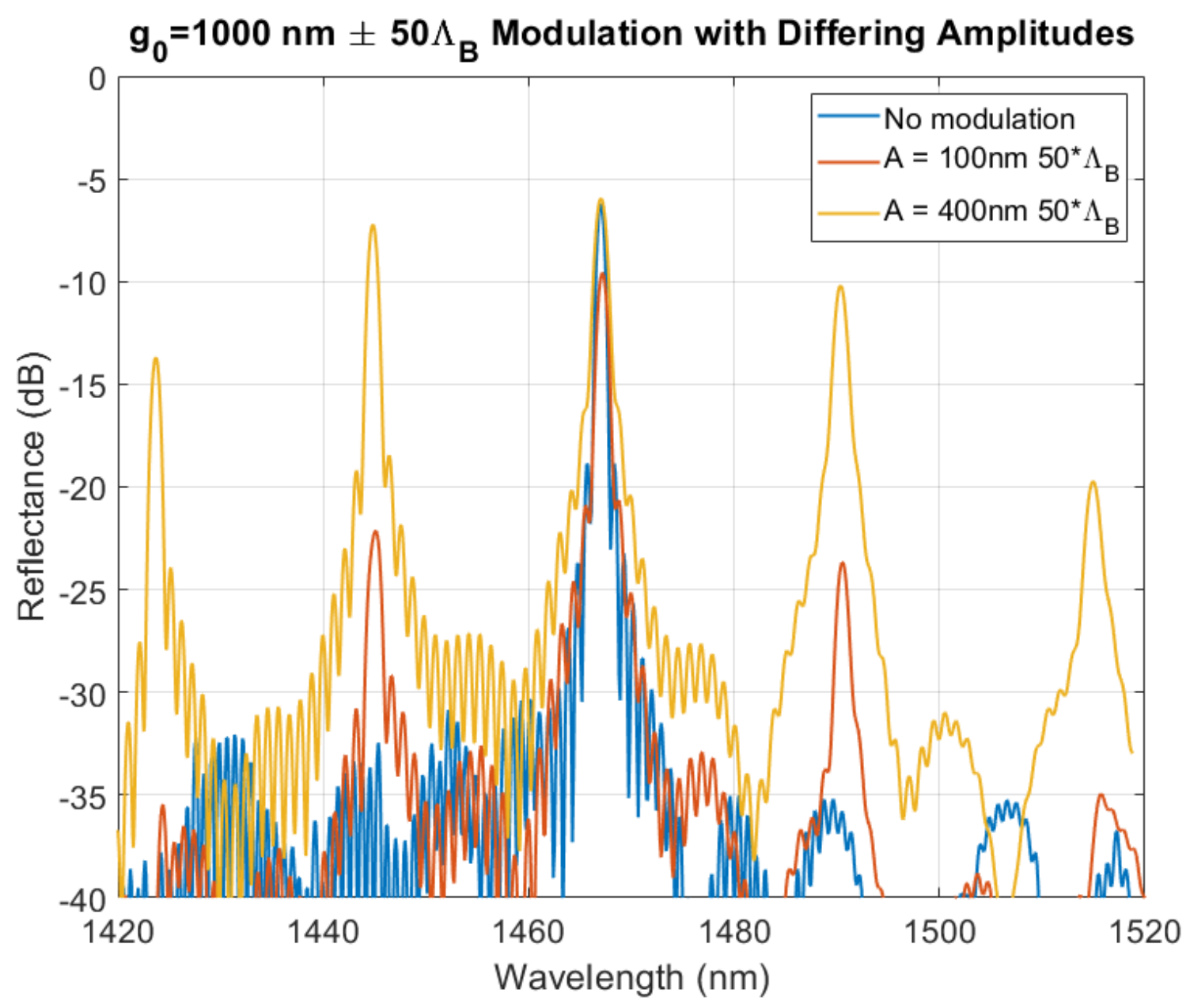

Figure 3.12: Reflectance spectra of an unmodulated TM-SWG grating compared to two modulations with different amplitudes.

the bandwidth of all peaks, this offset greatly reduces the maximum reflectance of each peak, often suppressing all but the central Bragg peak. Given that the goal of this thesis work was to investigate the effects of sidelobes peaks, only a small number of test filters incorporated offsets. The effect of this offset is shown in the simulation of an unmodulated grating in Fig. 3.13. 


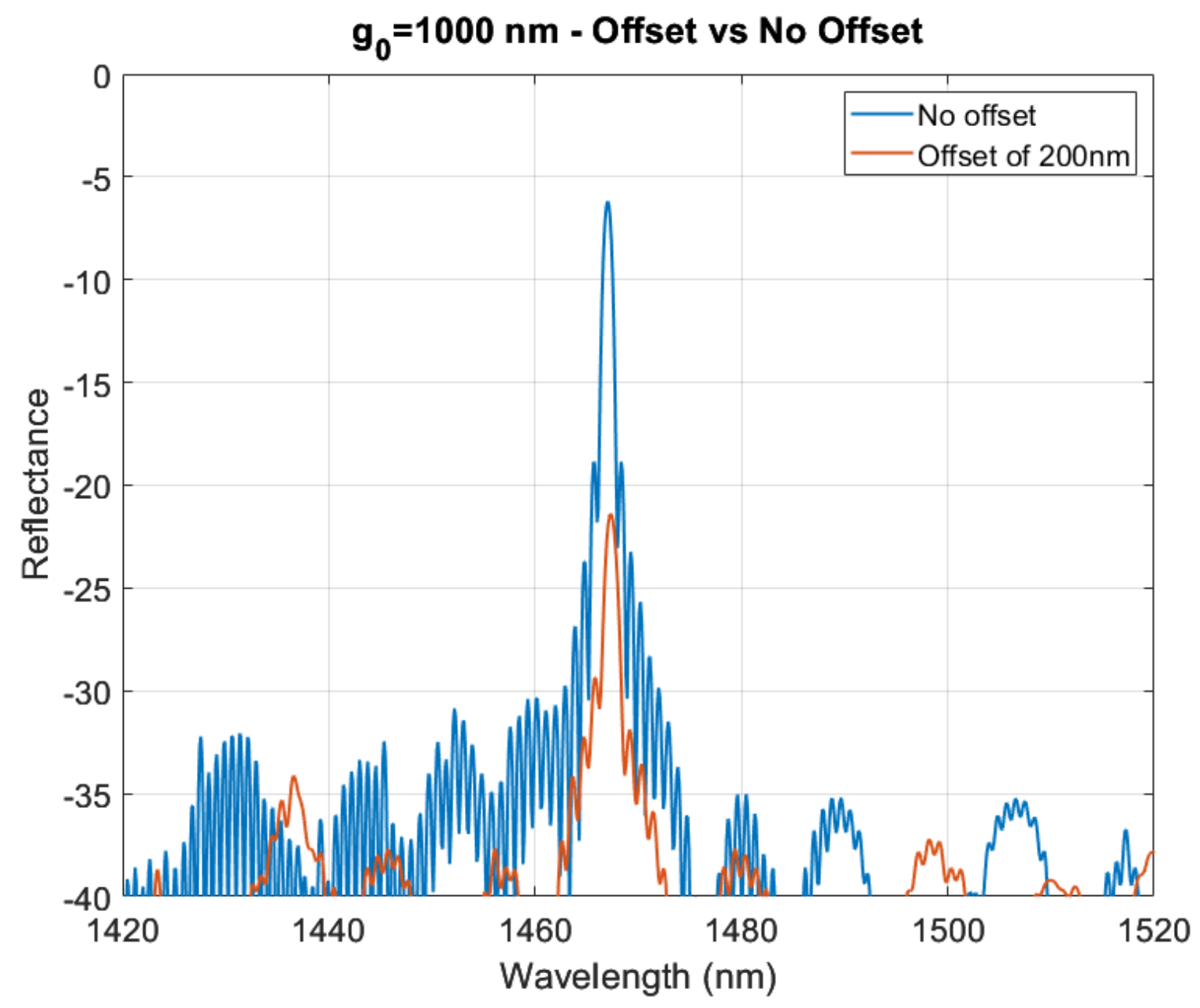

Figure 3.13: Reflectance spectra of an unmodulated grating with and without and offset.

Fig. 3.14 shows an FDTD simulation of two grating each with two superimposed cosine modulations. The FDTD spectra demonstrate the same dominant grouping of three reflection peaks as predicted by the earlier FFT in Fig. 3.10. 


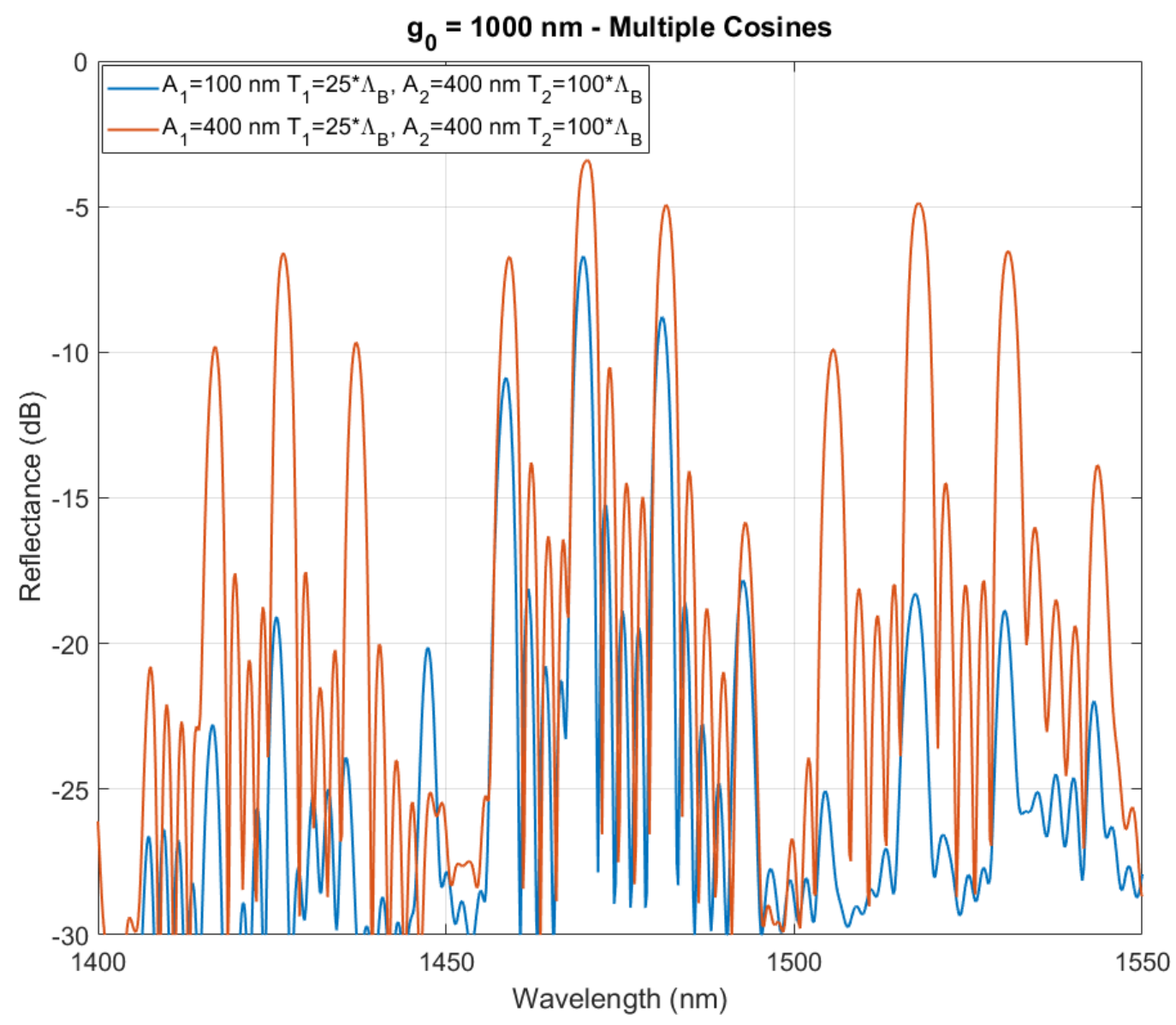

Figure 3.14: Reflectance spectra of a TM-SWG grating with multiple superimposed cosine modulations of different amplitudes.

\subsection{Thermal Modulation}

Thermal effects have been widely explored in silicon photonics due to the large thermooptic response of silicon's refractive index. Silicon has a thermo-optic coefficient $(d n / d T)$ of $1.86 \times 10^{-4} / K$, meaning an increase of a few Kelvin can shift the effective index on the scale of $10^{-3}$. In particular, a uniform shift in the temperature of a Bragg grating results in 
a shift of the Bragg wavelength, which makes them useful as passive temperature sensors. On the other hand a heater element can be fabricated above a Bragg grating in order to tune the local effective index, creating a dynamically tunable notch filter that controls the Bragg wavelength. This latter concept can be extended even further by implementing a nonuniform heater element in order to modulate the grating strength, much in the same way the variable pillar gap does. The temperature modulation could be used to dynamically induced peak splitting, with the applied voltage controlling the height of the secondary peaks.

\subsubsection{Lumerical HEAT Simulations}

Lumerical HEAT was used to simulate the thermal response of the square-wave heater pictured in Fig. 3.15. The square-wave shape of the heater is intended to produce a periodic temperature gradient. The heater is made of a resistive metal that rises in temperature due to Joule heating when a current is supplied. A titanium-tungsten alloy (sheet resistance of $3.07 \Omega / s q[32]$ ) was used as the heater element and the corresponding material data was entered into the simulation parameters (see section 3.7 for details on

fabrication). The difference in temperature between the heater layer and the waveguide layer is generally a few degrees, and the waveguide is so small when compared to the heater that $x$ dimension can be taken as uniform. 
a)

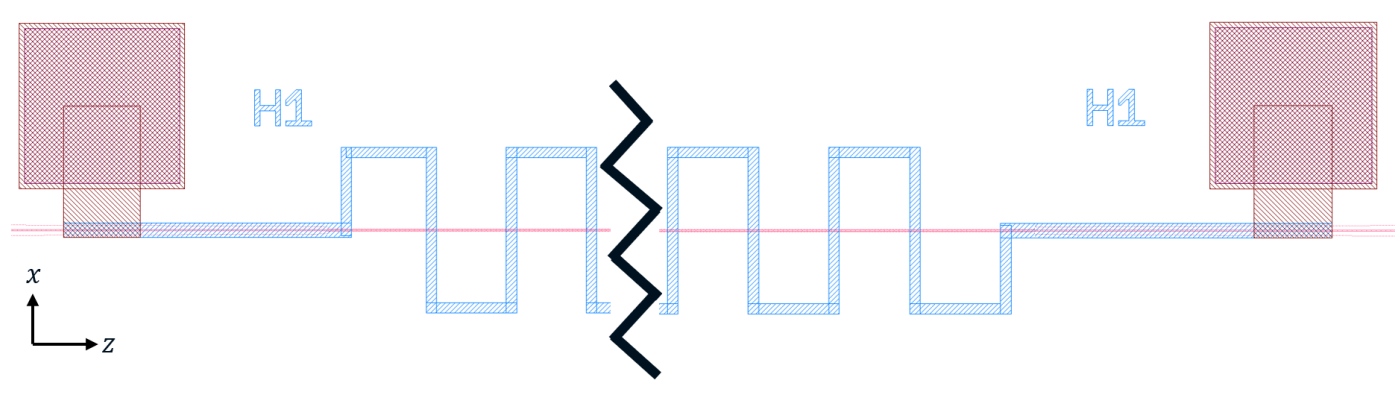

b)

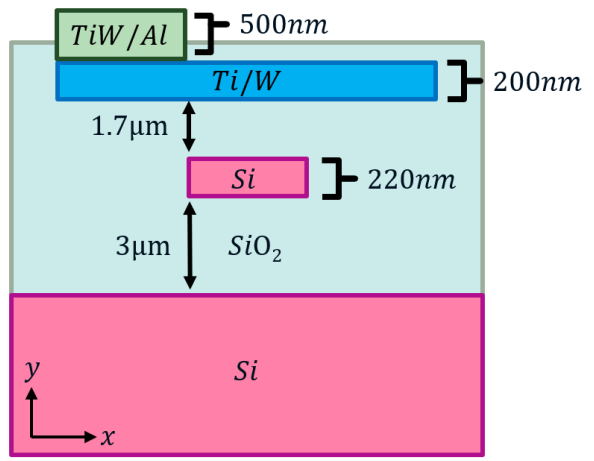

c)

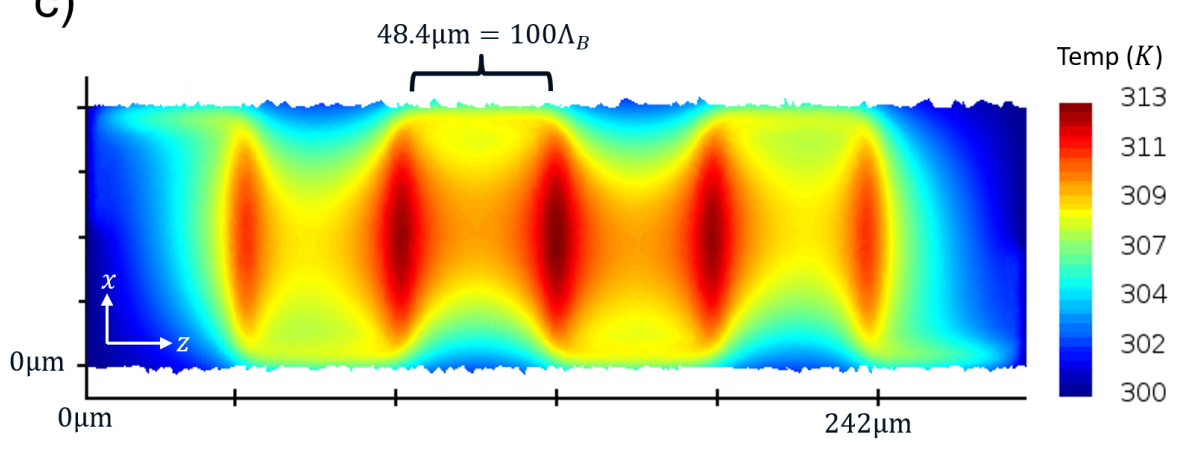

Figure 3.15: a) Periodic heater layout (truncated in length). Blue: Titanium-Tungsten heater element. Pink: Silicon Bragg grating. Brown: Electrode pads. b) Cross-sectional view of fabrication process with heater element. c) Temperature distribution due a voltage boundary condition applied to the simulation

\subsubsection{D FDTD Simulations}

Lumerical FDTD can import temperature profiles simulated in HEAT and incorporate them into the FDTD simulation. This is done by defining two perturbation materials based on silicon and silicon dioxide, and setting their $d n / d T$ coefficients to $1.86 \times 10^{-4} / K$ and $1 \times$ $10^{-5} / K$, respectively. The reference filter was a wire waveguide with TM input, $g_{0}=350$ $\mathrm{nm}$, and a length of 1000 Bragg periods. With no modulation (i.e. uniform temperature of $300 \mathrm{~K}$ ) this filter produces a central Bragg peak at $1615 \mathrm{~nm}$. Fig. 3.16 shows the resulting 
peak splitting due to a heater with a spacing of $100 \Lambda_{B}$.

a)

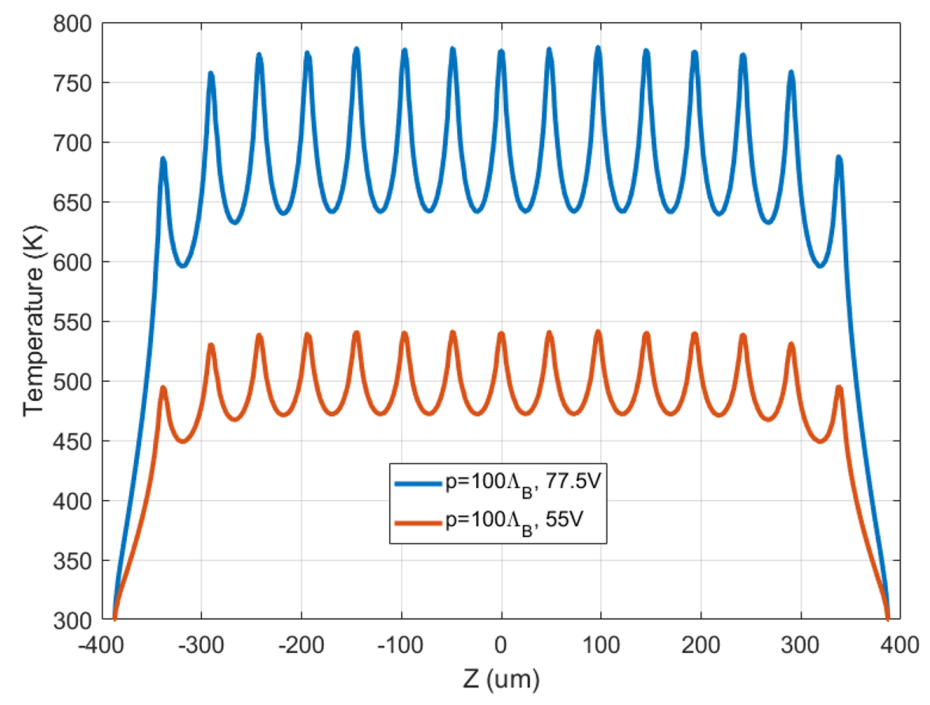

b)

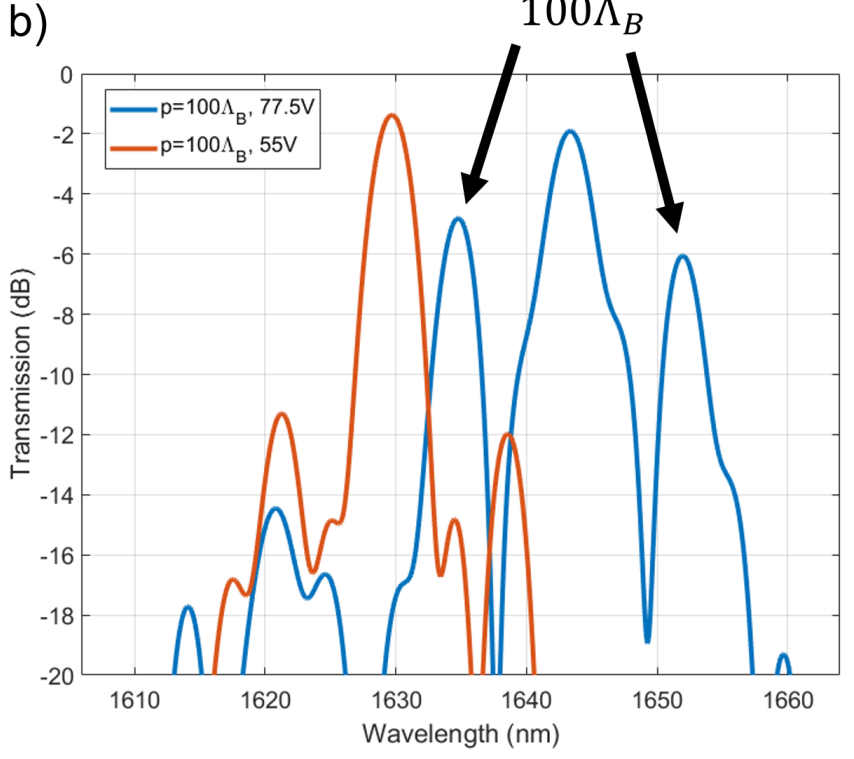

Figure 3.16: A heater with a spacing of $100 \Lambda_{B}$ was simulated for 16 periods: a) Temperature modulation along the waveguide for different input voltages, b) Resulting FDTD simulation spectra.

There are two parameters of interest to consider, the maximum temperature swing $(\Delta T)$, and the average background temperature $\left(T_{a v}\right)$. These can be considered analogous to $g_{i}$ and $g_{0}$ in the passive filters, with $\Delta T$ and its period determining the size and position of the sidelobes, and $T_{a v}$ determining the shift in the central Bragg wavelength $\lambda_{B}$. However, whereas $g_{i}$ and $g_{0}$ could be largely set independently (limited only by the minimum feature size), the size of $\Delta T$ is linked to the heater spacing and $T_{a v}$. For the temperature modulation in Fig. 3.16 the maximum $\Delta T$ was $134.2 \mathrm{~K}$ and $67.5 \mathrm{~K}$ for the $77.5 \mathrm{~V}$ and $55 \mathrm{~V}$ inputs, respectively. However this was in addition to a change in $T_{a v}: 701 \mathrm{~K}$ and $508 \mathrm{~K}$ respectively. So although $\Delta T$ is relatively large, the average background temperature is quite hot as a direct consequence, which would almost certainly put constraints on any test setup and 
operational range.

Further simulations have shown that smaller periods perform worse than larger periods, as smaller periods required larger input voltages to achieve the same $\Delta T$, while also significantly increasing $T_{a v}$. This can be seen in Fig. $3.17 \mathrm{a}$ ), where the same number of periods and same input voltages were tested for a heater with spacing of $50 \Lambda_{B}$. The resulting $\Delta T$ were similar in scale $(122.4 \mathrm{~K}$ and $60.8 \mathrm{~K})$, but had much higher $T_{a v}$ values $(1382.9 \mathrm{~K}$ and $845.4 \mathrm{~K})$. These temperatures were approaching the melting point of silicon and would likely degrade the device performance.

The same number of periods and input voltages were simulated for a heater with spacing of $500 \Lambda_{B}$ (as in Fig. 3.17) and the opposite trend was observed. The resulting $\Delta T$ were 39.6 $\mathrm{K}$ and 19.9 $\mathrm{K}$, but the $T_{a v}$ values of $331.4 \mathrm{~K}$ and $315.8 \mathrm{~K}$ were much more reasonable. The limiting factor in this case is the large size of the device. To achieve the same number of heater periods (i.e. 16), the device length is ten times as long as the $50 \Lambda_{B}$ case, and the heater resistance scales with length. The heater design studied in this work is an inefficient squarewave structure and could very likely be improved upon to decrease the total resistance. For instance, the heater elements could be interlaced with lower resistance metal-interconnecting layers such that the heater is only included when crossing the waveguide and not parallel to it. 
a)

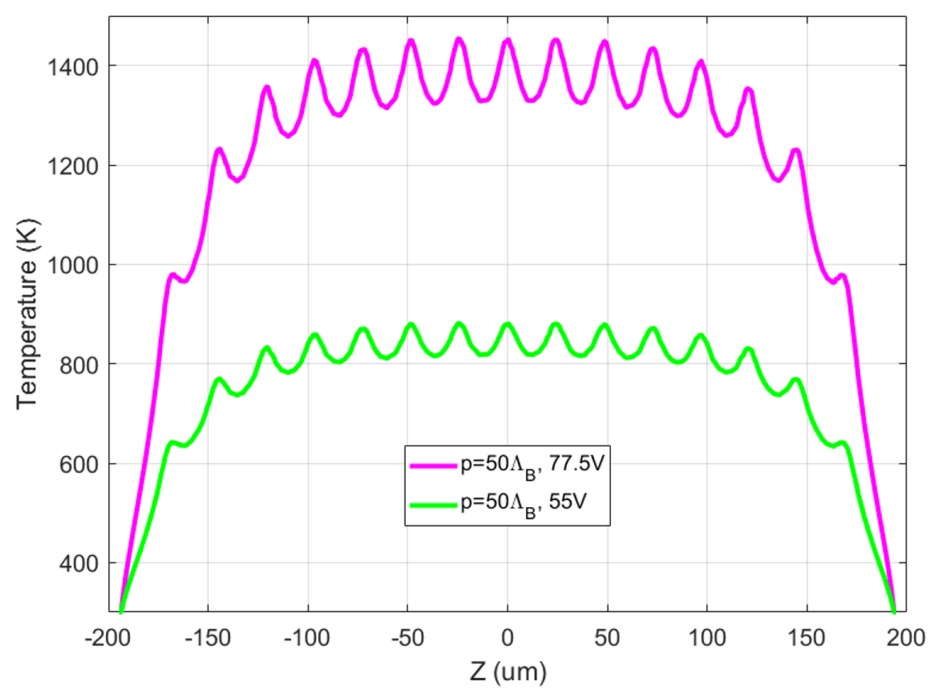

b)

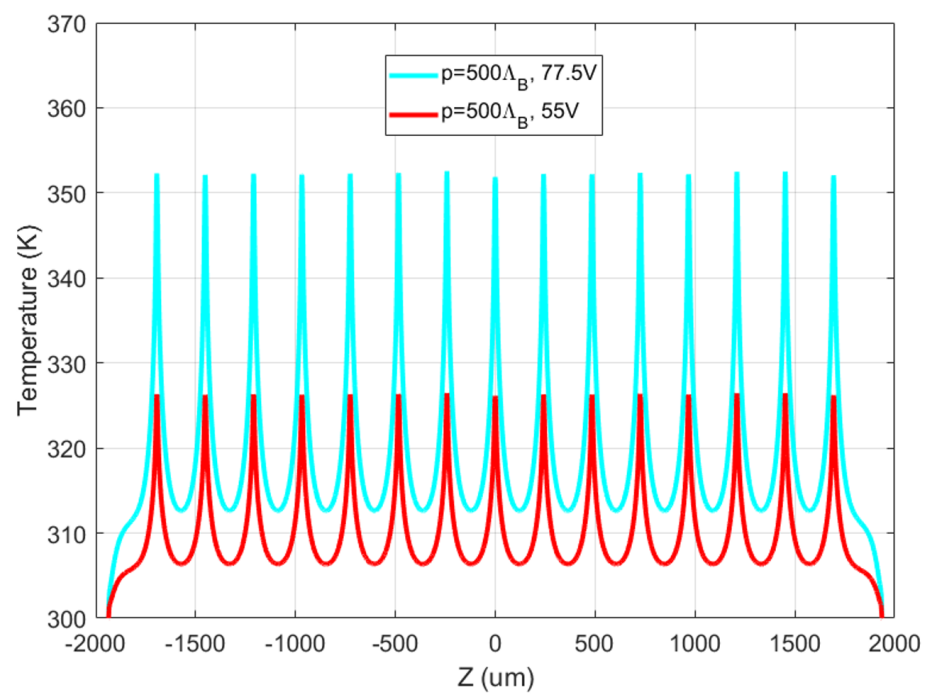

Figure 3.17: Temperature modulation for a heater with 16 periods and a spacing of a) $50 \Lambda_{B}$, b) $500 \Lambda_{B}$

\subsection{Layout \& Fabrication}

Fabrication was carried out by Applied Nanotools using their nanophotonic foundry service [32]. The filters were fabricated on an SOI substrate with a $220 \mathrm{~nm}$ device layer and a 3 $\mu m$ thick buried-oxide (BOX) layer. Previous attempts at fabricating SWG Bragg filters on a $2 \mu \mathrm{m}$ BOX layer led to high levels of loss due to the loosely confined modes. Devices were patterned using $100 \mathrm{keV}$ electron-beam-lithography with a minimum silicon feature size of $60 \mathrm{~nm}$, followed by the application of an upper-cladding of $\mathrm{SiO}_{2}$ via chemical vapour deposition. The gratings have a total length of $8.78 \mathrm{~mm}$ with a $250 \mu \mathrm{m}$ deep-trench etch along the perimeter for use with metamaterial edge-couplers (edge-coupler design previously implemented in [23]). The input and output sections (1000 Bragg periods) were apodized 
by tilting the row of loading segments by 0.25 degrees (as in [33]), gradually decreasing the gap to the nominal value of $g_{0}$. This apodization helps reduce the unwanted sidelobes of the central Bragg peak by gradually increasing the grating strength from zero up to its nominal value of $\kappa_{0}$. The modulated section is comprised of approximately 15500 Bragg periods.

A variety of Bragg grating variants were fabricated, including both SWG-core and wirecore variants, each with three values of $g_{0}$ upon which several modulation schemes were imposed. The SWG variants included $g_{0}$ values of $500 \mathrm{~nm}, 750 \mathrm{~nm}$ and $1000 \mathrm{~nm}$, and the wire variants included $g_{0}$ values of $180 \mathrm{~nm}, 225 \mathrm{~nm}$ and $350 \mathrm{~nm}$. Heater elements were implemented on select filters with a $200 \mathrm{~nm}$ thick Ti/W allow layer and $500 \mathrm{~nm}$ thick TiW/Al routing bilayer. The top $\mathrm{SiO}_{2}$ passivation layer covers the Ti/W heaters but is etched away in specific locations to access the electrode pads for the heaters. The heater layer is situated in the upper cladding $1.7 \mu \mathrm{m}$ above the waveguides as in Fig. $3.15 \mathrm{~b}$ ). Several calibration waveguides of different lengths were included on the layout to quantify the propagation loss of both the SWG and wire variants. Scanning-electron microscope (SEM) pictures of the fabricated Bragg filters are shown in Fig. 3.18. 


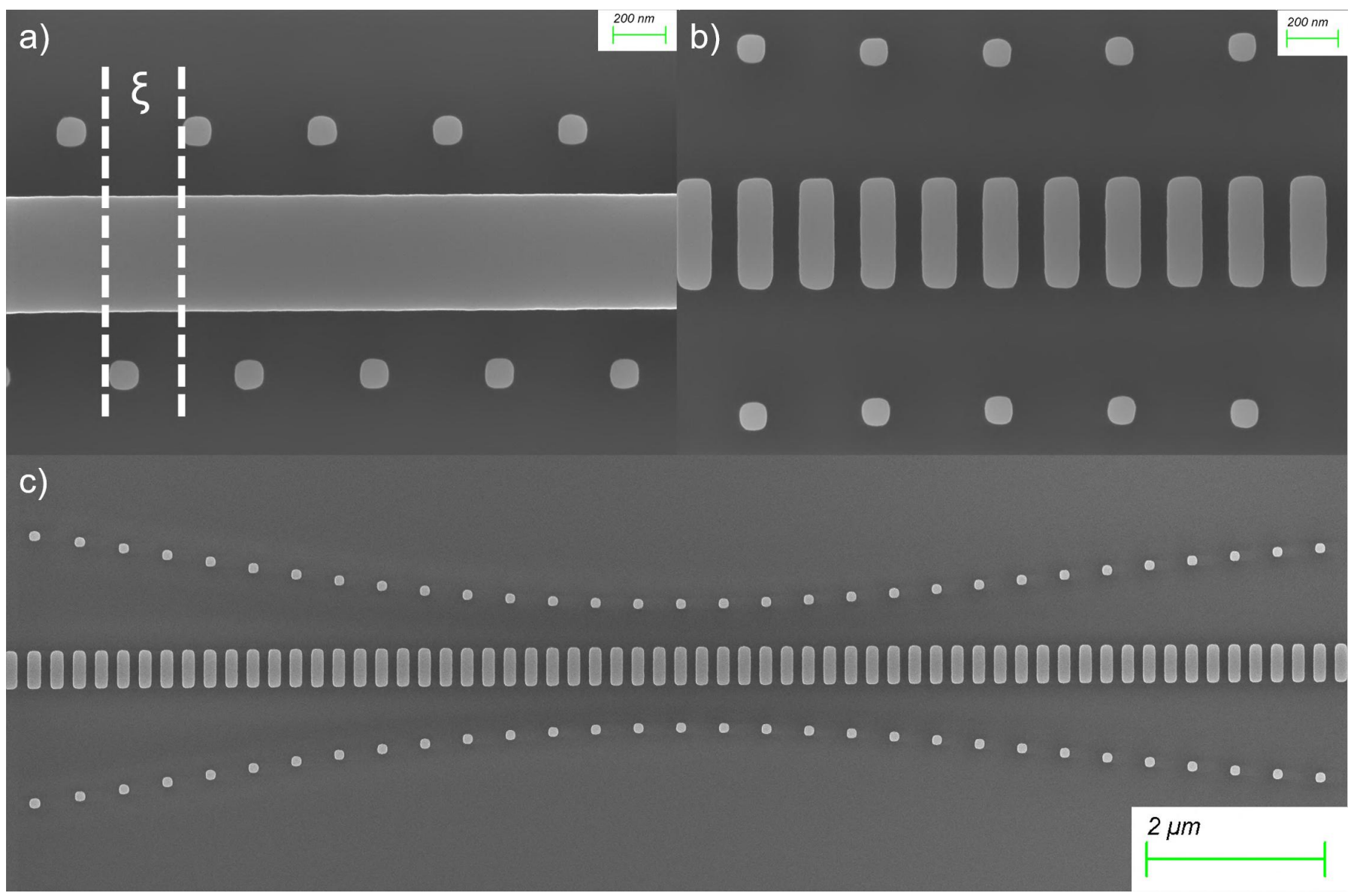

Figure 3.18: SEM pictures of the fabricated Bragg filters: a) A wire-core filter with a constant offset $\xi$ of $200 \mathrm{~nm}$. b) An SWG-core filter with a cosine modulation. c) A zoomedout view of the previous SWG filter showing a full period of the modulation.

\subsection{Testing \& Measurement Results}

Grating performance was tested at the National Research Council facility in Ottawa by performing a transmission sweep using a tunable laser source. The chip was patterned with a $250 \mu \mathrm{m}$ deep trench etch along the perimeter to enable coupling using metamaterial edge couplers. 


\subsubsection{Propagation Loss}

Several serpentine waveguide structures of varying lengths were included on the layout to calculate the propagation loss. The waveguides have the same dimensions as the Bragg grating cores but without the loading segments. Each calibration waveguide consisted of four $180^{\circ}$ waveguide bends with a radius of $12.5 \mu \mathrm{m}$, and two edge couplers. In the case of the SWG waveguides a fishbone taper was used to convert to a standard wire waveguide before the waveguide bends, as the loosely confined SWG mode would require a significantly larger radius. Fig. 3.19 shows the average transmission loss within the wavelength range of 1470-1580 nm plotted as a function of propagation length.

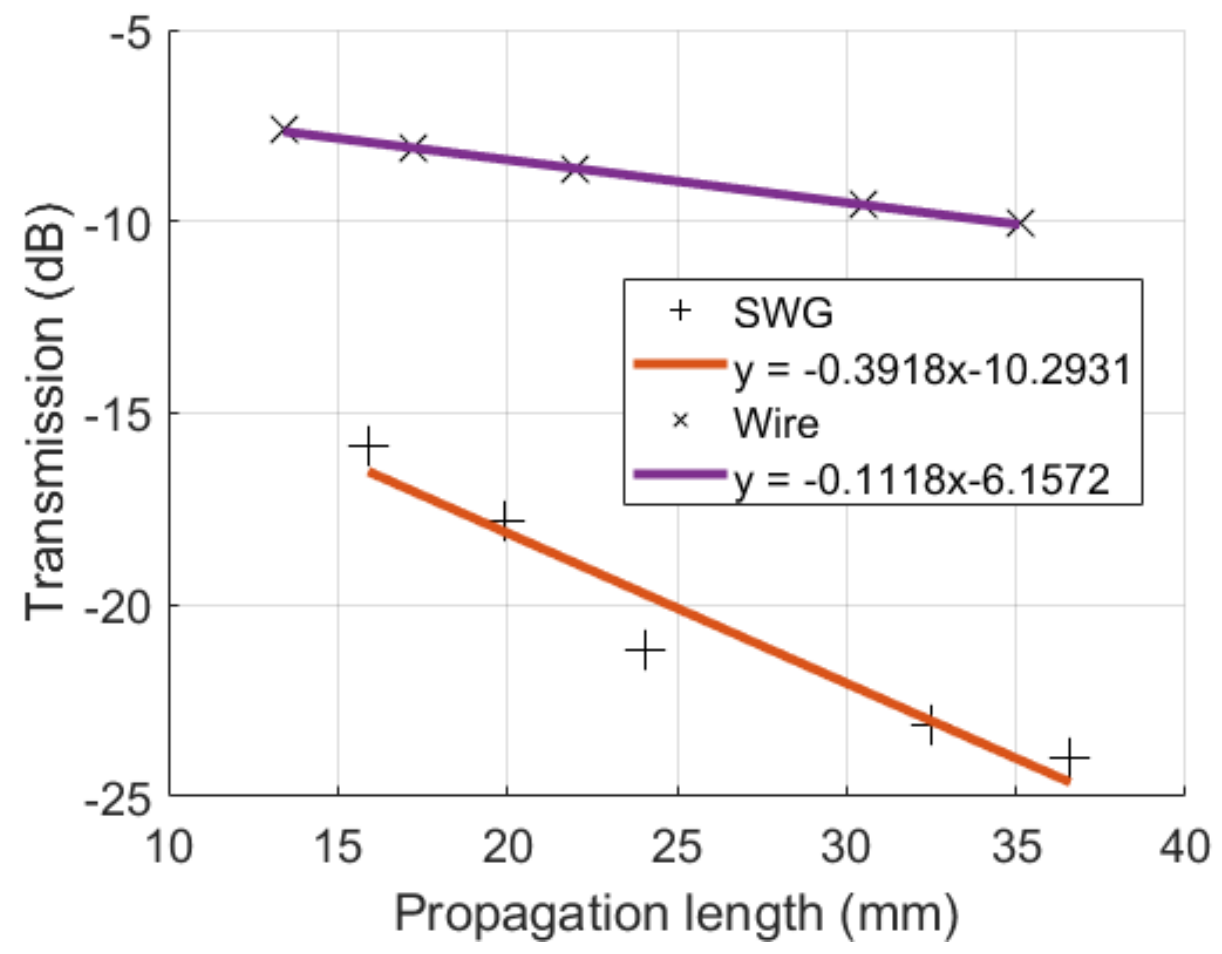

Figure 3.19: Average measured TM-input across the wavelength range of 1470-1580, plotted as a function of propagation length. 
A linear-fit was applied to each data-set and the resulting slopes correspond to the propagation loss. The wire-calibration loss structures gave a fit of $0.112 \mathrm{~dB} / \mathrm{mm}$, whereas the SWG structures gave a fit of $0.396 \mathrm{~dB} / \mathrm{mm}$. As anticipated the $\mathrm{SWG}$ mode is much lossier than the wire mode due to the comparatively low confinement.

\subsubsection{Passive Filter Performance}

Fig. 3.20 shows three unmodulated SWG filters with TM input, as well as the $g=500 \mathrm{~nm}$ variant with an offset of $200 \mathrm{~nm}$. They produce one strong central peak, and the smaller gaps result in a larger bandwidth. The null-to-null bandwidth of the filters are $0.68 \mathrm{~nm}, 1.92$ $\mathrm{nm}$, and $3.88 \mathrm{~nm}$, for the $1000 \mathrm{~nm}, 750 \mathrm{~nm}$, and $500 \mathrm{~nm}$ gaps, respectively. The $500 \mathrm{~nm}$ gap variant with the offset lowers the bandwidth further from $3.88 \mathrm{~nm}$ to $1.36 \mathrm{~nm}$ with no change in the center wavelength. Decreasing the gap from $1000 \mathrm{~nm}$ to $500 \mathrm{~nm}$ shifts $\lambda_{B}$ up from $1449.04 \mathrm{~nm}$ to $1450.72 \mathrm{~nm}$, a change of $1.68 \mathrm{~nm}$. 


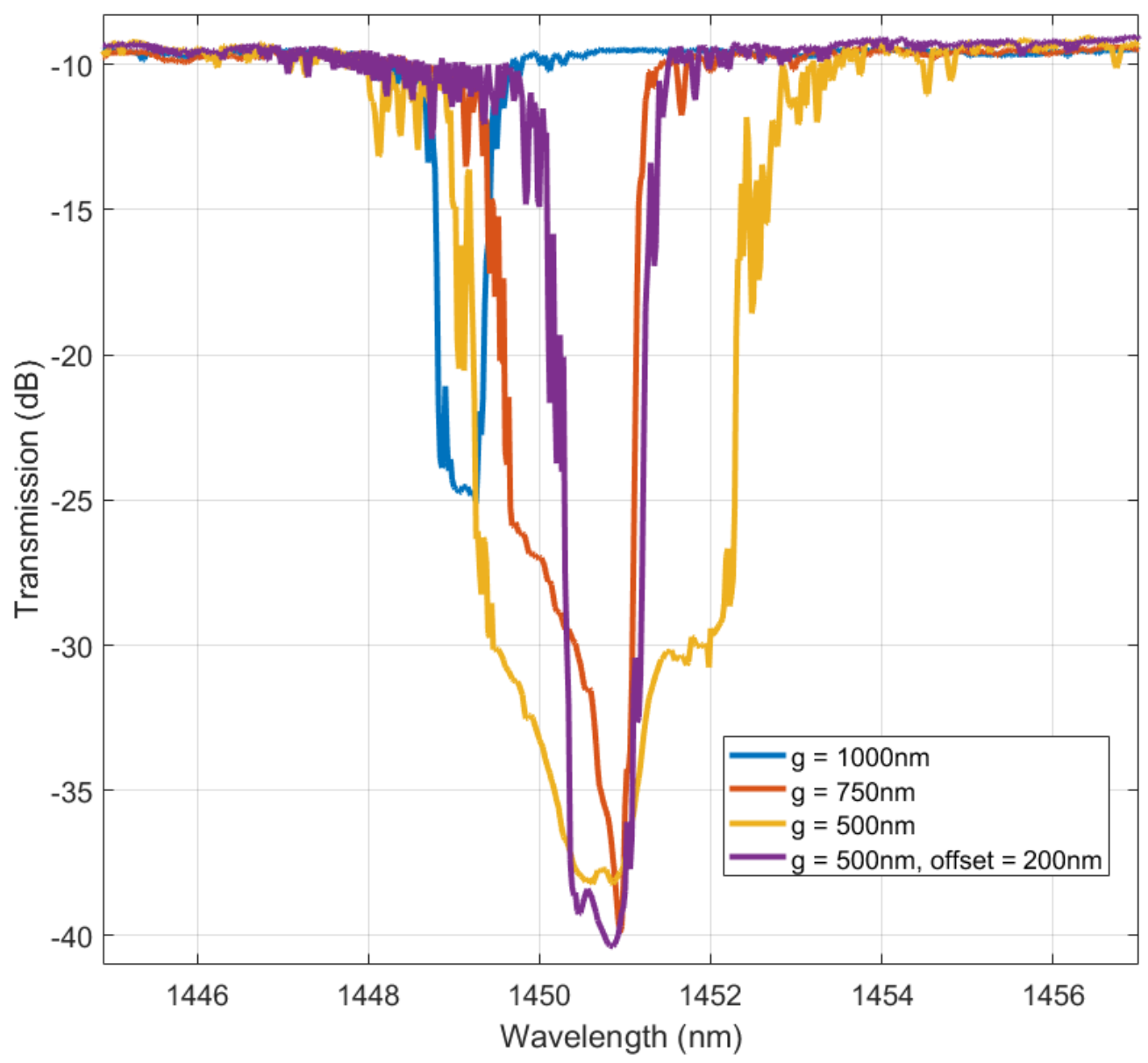

Figure 3.20: Transmission spectra for unmodulated SWG-filters with TM input.

Fig. 3.21 shows three SWG gratings with a gap of $500 \mathrm{~nm}$, one unmodulated and the other two with multiple cosine modulations. In particular the peak contributed by the $80 \Lambda_{B}$ modulation period can be suppressed simply by omitting it from the modulation. It can be seen that through the inclusion or omission of specific cosine periods any arbitrary spectrum can be engineered. 


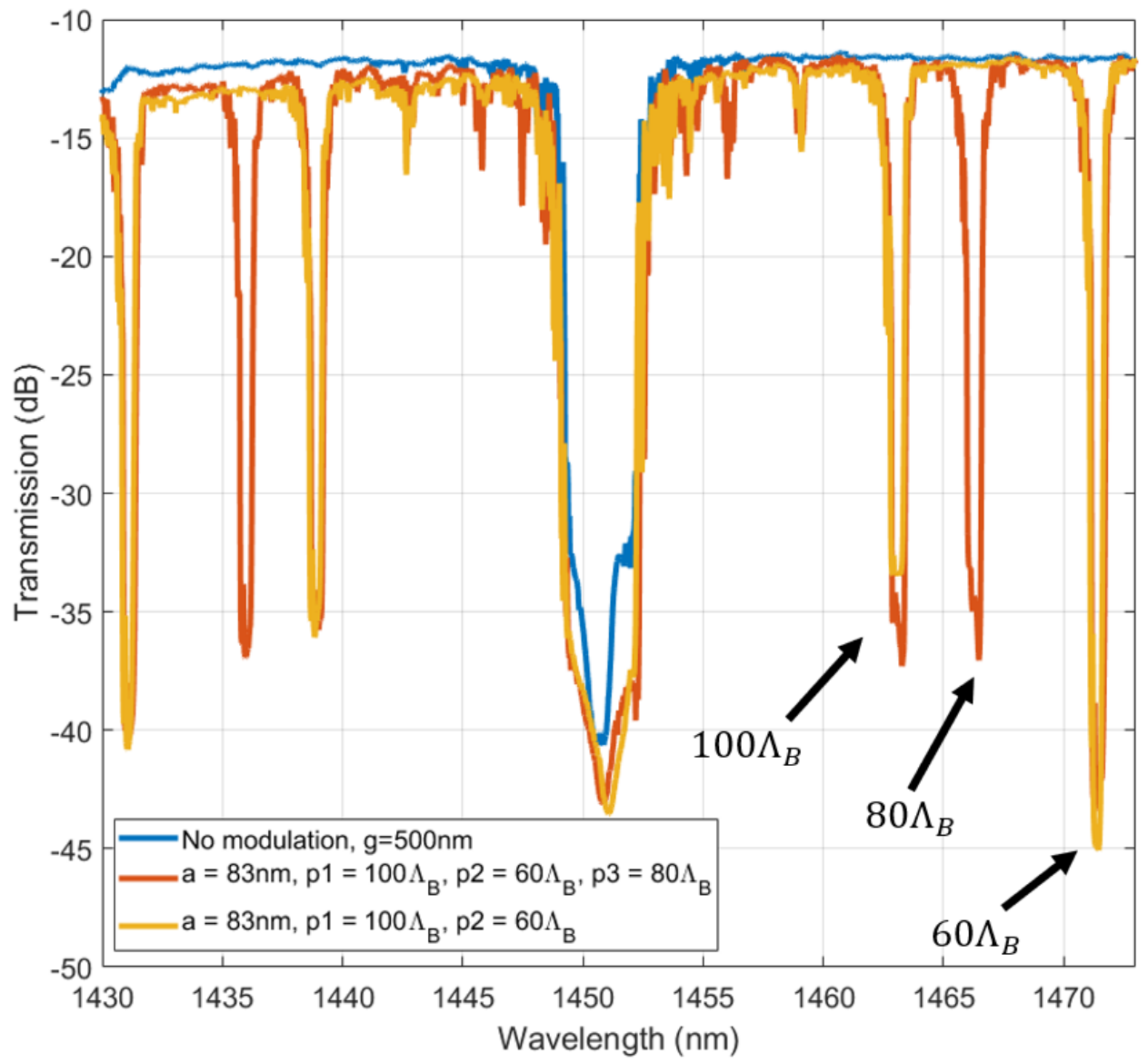

Figure 3.21: Transmission spectra for SWG gratings with multiple cosine modulations.

Fig. 3.22 shows three SWG gratings with a gap of $500 \mathrm{~nm}$, one unmodulated and the other three with the same cosine period but varying amplitudes. It can be seen that the central peak is largely unaffected by the amplitude of the modulation, but the height and bandwidth of the sidelobes is highly sensitive. This dependence is most obvious in the rightmost peak, which is a $2^{\text {nd }}$ order sidelobe. There is a significant drop-off in extinction ratio for higher order sidelobes, and strong modulation amplitudes are required to produce $3^{\text {rd }}$ order and higher sidelobes. 


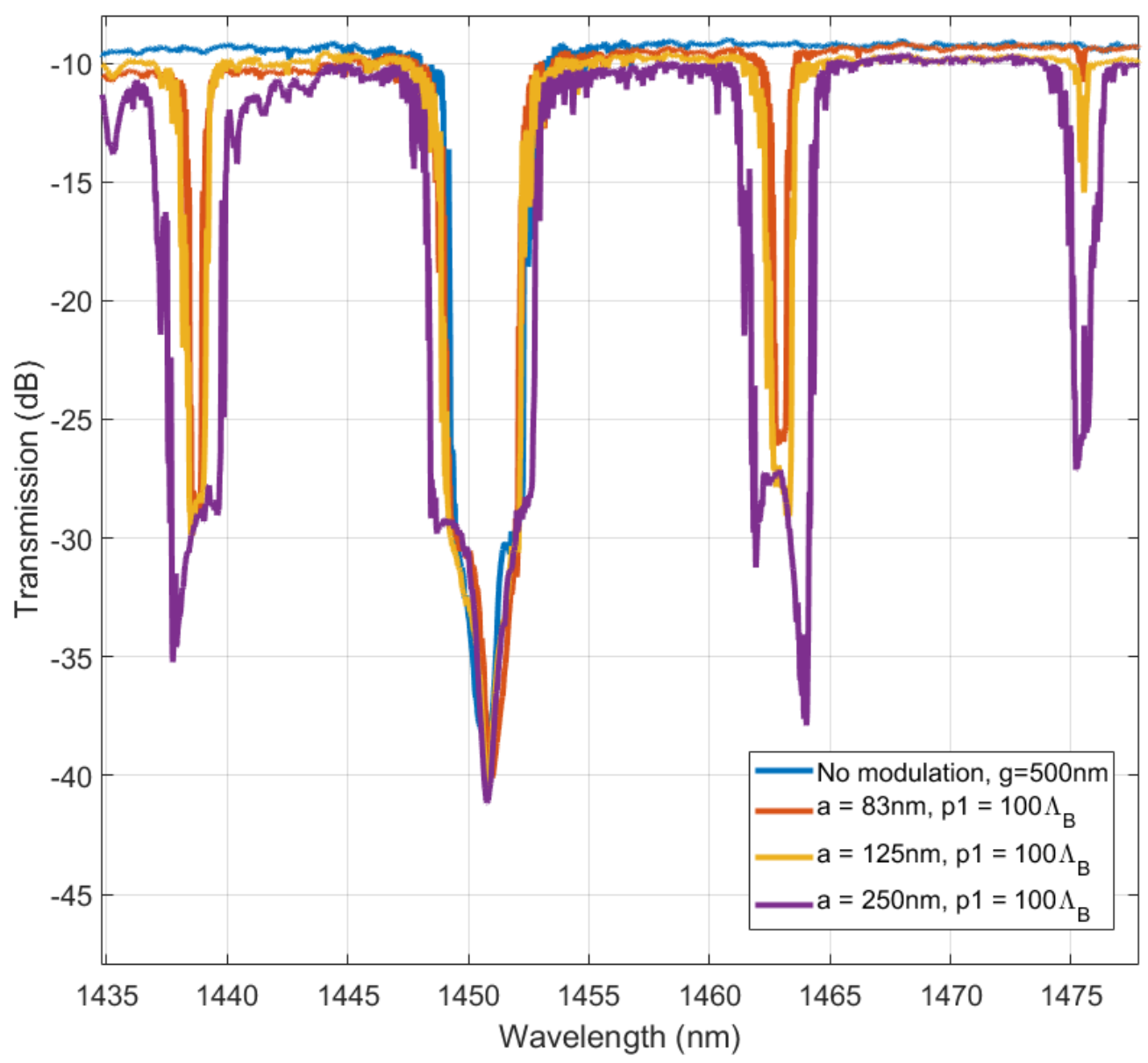

Figure 3.22: Transmission spectra for SWG gratings with variable amplitude modulations.

Fig. 3.23 shows three unmodulated wire filters with TM input. They produce one strong central peak, and the smaller gaps result in a larger bandwidth. The null-to-null bandwidth of the filters are $2.17 \mathrm{~nm}, 4.50 \mathrm{~nm}$, and $5.06 \mathrm{~nm}$, for the $350 \mathrm{~nm}, 225 \mathrm{~nm}$, and $180 \mathrm{~nm}$ gaps, respectively. The $180 \mathrm{~nm}$ gap variant with the offset lowers the bandwidth further from 5.06 $\mathrm{nm}$ to $1.68 \mathrm{~nm}$ with no change in the center wavelength. Decreasing the gap from $350 \mathrm{~nm}$ to $180 \mathrm{~nm}$ shifts $\lambda_{B}$ up from $1613.96 \mathrm{~nm}$ to $1615.24 \mathrm{~nm}$, a change of $1.28 \mathrm{~nm}$. 


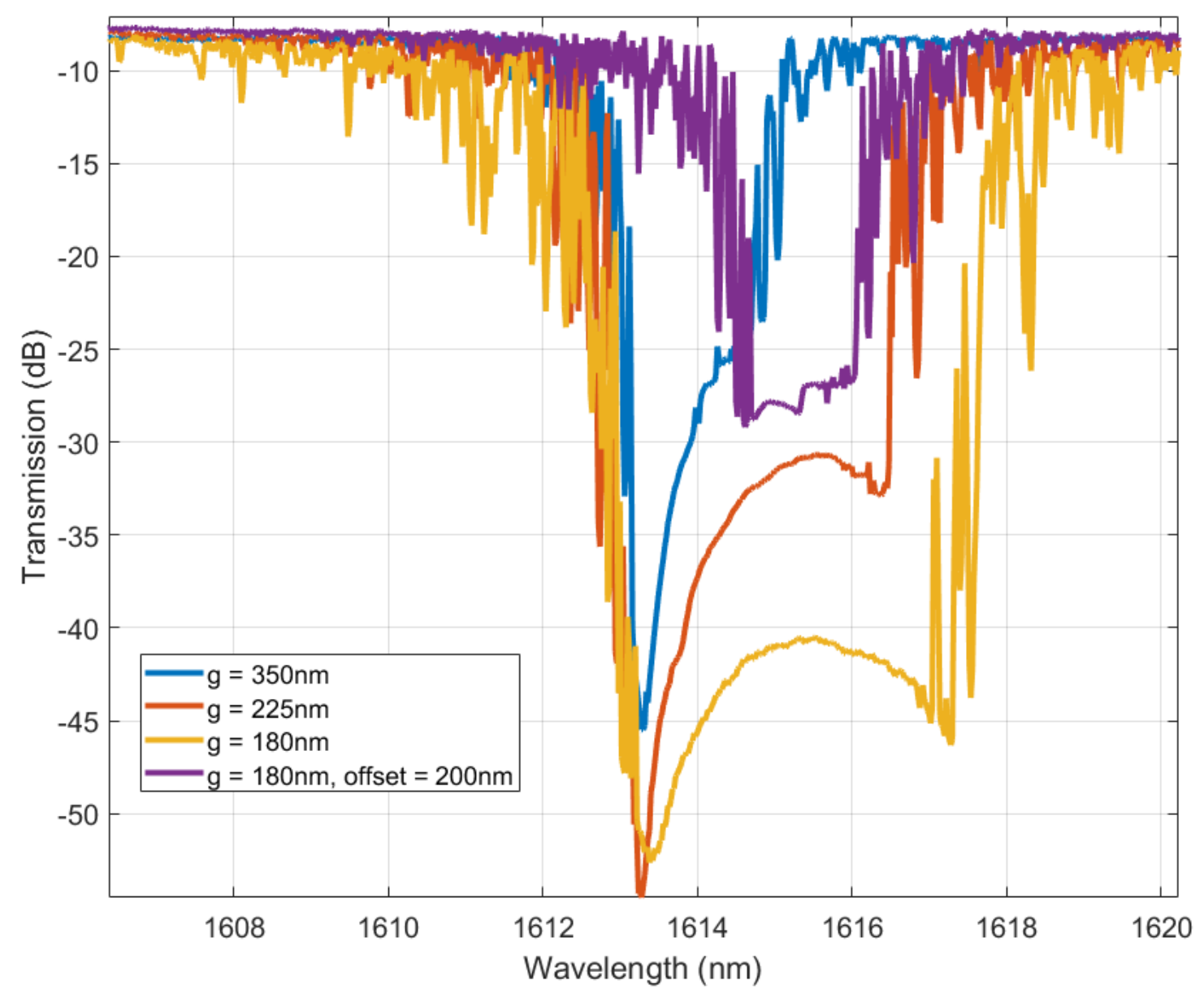

Figure 3.23: Transmission spectra for unmodulated wire-filters with TM input.

Fig. 3.24 shows three wire gratings with a gap of $180 \mathrm{~nm}$, one unmodulated and the other two with multiple cosine modulations. Just as for the SWG gratings the the peak contributed by the $80 \Lambda_{B}$ modulation period can be suppressed simply by omitting it from the modulation. When compared to the SWG filters the sidelobes are not as clearly defined and subject to phase jitter due to the field concentration along the wire-core's sidewalls. 


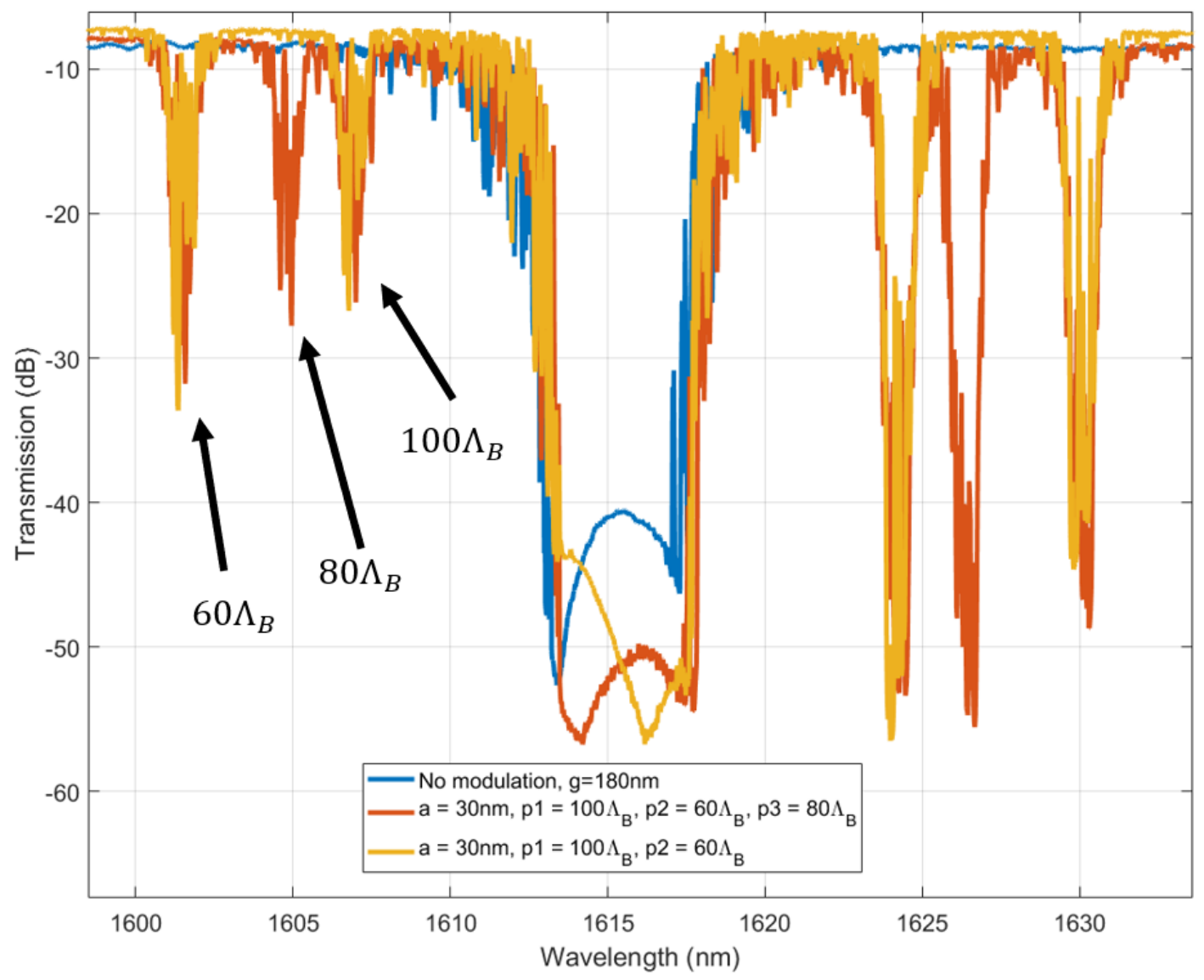

Figure 3.24: Transmission spectra for wire gratings with multiple cosine modulations.

Fig. 3.25 shows three wire gratings with a gap of $180 \mathrm{~nm}$, one unmodulated and the other two with the same cosine period but varying amplitudes. Much like the SWG filters the central peak is largely unaffected by the amplitude of the modulation, but the height and bandwidth of the sidelobes is highly sensitive. One key difference is that the wire filter sidelobes appear to be much more asymmetric, with $2^{\text {nd }}$ and even $3^{\text {rd }}$ order sidelobes visible on the right-hand side of the central peak, but only a $1^{\text {st }}$ order sibelobe on the left-hand side. 


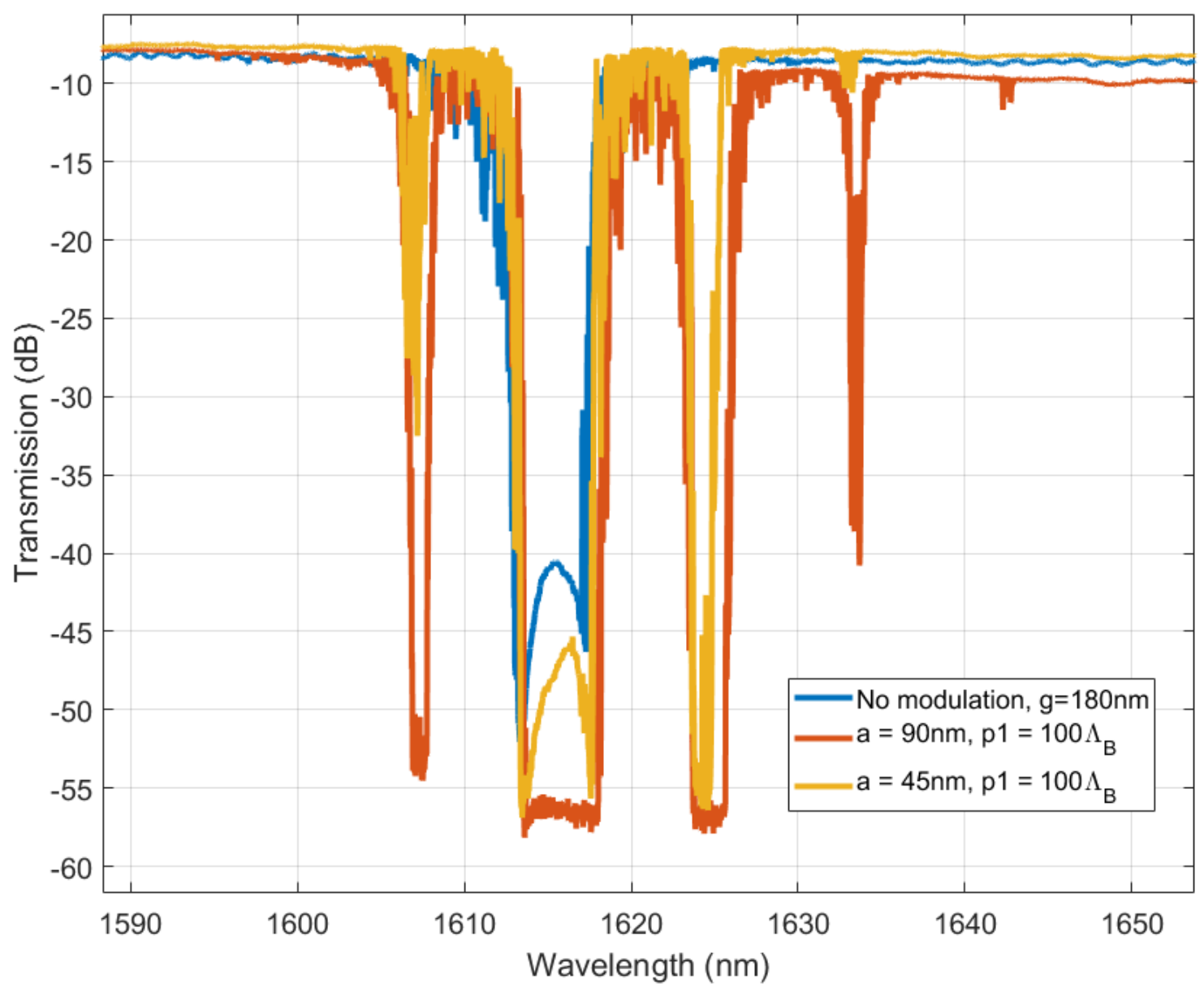

Figure 3.25: Transmission spectra for wire gratings with variable amplitude modulations.

\subsubsection{Thermal Filter Performance}

The thermally modulated filters did not successfully demonstrate dynamic peak splitting due to two critical limitations. The first limitation is the presence of the metal heaters causing the leakage of the TM SWG mode. The TM SWG mode is extremely lossy even without the metal heater present, producing the transmission spectra in Fig. 3.26. The response is 
that of an unintentional resonating cavity created by the change in index when the heater switches from parallel to perpendicular.

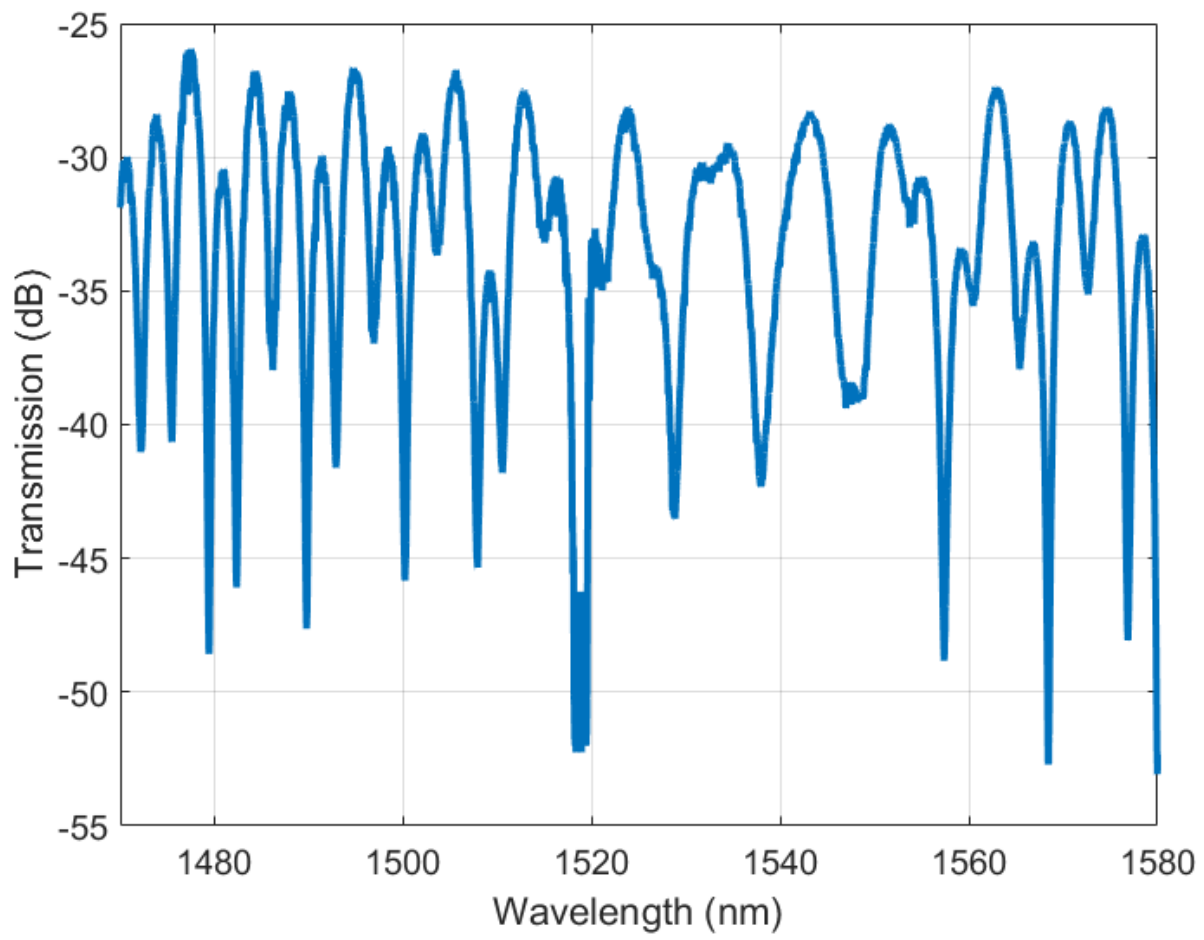

Figure 3.26: Transmission spectra for a TM SWG filter with a heater element $1.7 \mu m$ above the waveguide.

This phenomenon was not present in the TM wire filters, as that mode profile is much more tightly confined to the waveguide core. However no peak splitting was demonstrated as the periodic heater element has an extremely high resistance (approximately $12.75 k \Omega$ ) due to the long length of the square-wave heater pattern. This resistance severely limits the maximum current that can be supplied for Joule heating. The experimental setup was able to supply an input of up to $76 \mathrm{~V}$, which produced an average chip temperature increase of $6{ }^{\circ} \mathrm{C}$. It can be seen in Fig. 3.27 that the central wavelength shifts by approximately $0.6 \mathrm{~nm}$ from 
$0 \mathrm{~V}$ to $76 \mathrm{~V}$, but with no observable peak splitting. Although a mild periodic temperature gradient would be induced, it is not a sufficiently large temperature swing to modulate the strength of the grating, and is effectively just a uniform temperature increase across the entire grating. Comparing to the temperature simulations in section 3.6.1, a temperature modulation needs to be on the scale of hundreds of degrees to produce observable sidelobes.

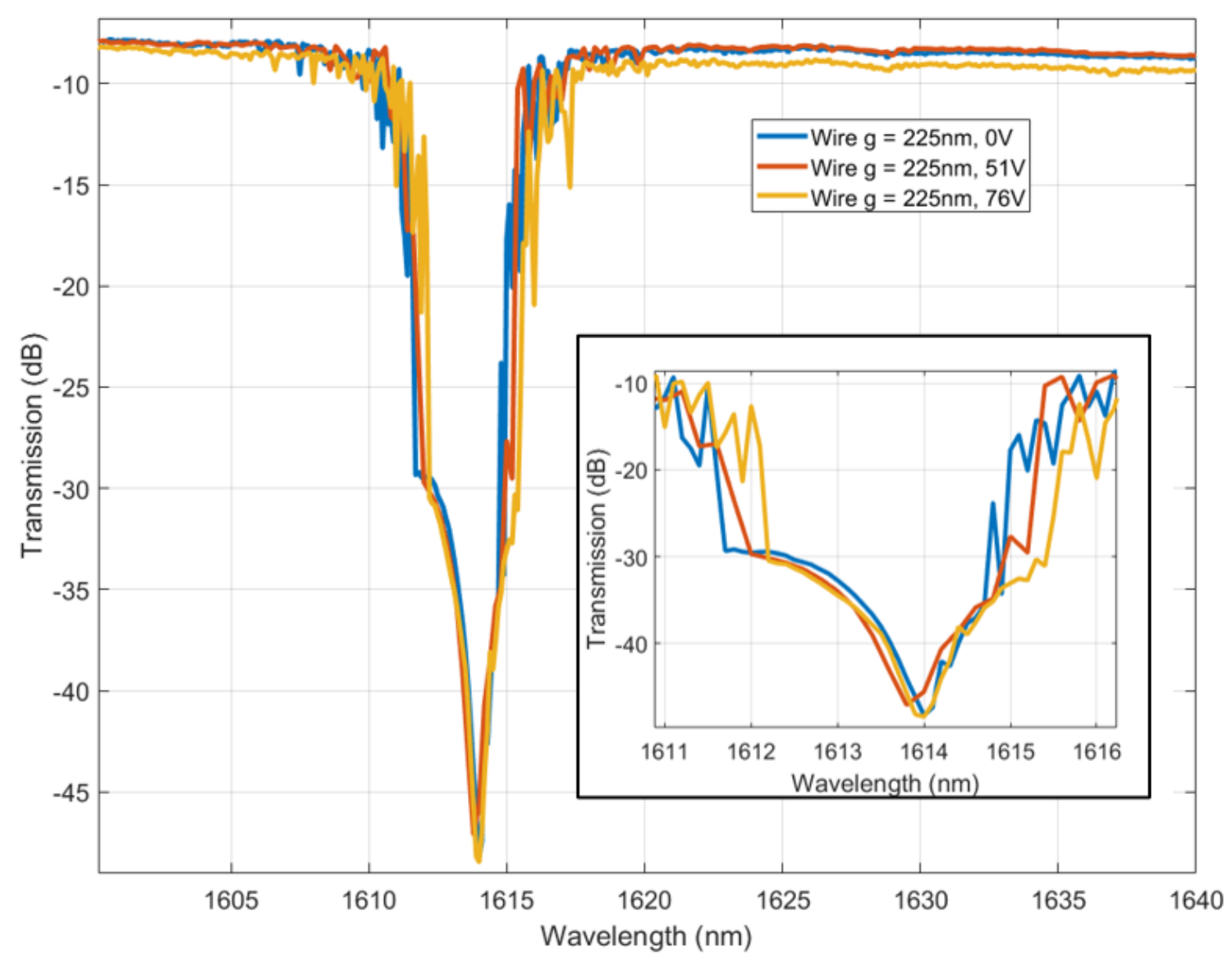

Figure 3.27: Transmission spectra for a TM wire filter when no voltage has been supplied versus when $51 \mathrm{~V}$ and $76 \mathrm{~V}$ has been supplied. The heater has a period of $100 \Lambda_{B}$ an approximate resistance of $12.75 \mathrm{k} \Omega$. 
3. Arbitrary Bragg Filter Sidelobe Engineering

\subsection{Summary}

This chapter has provided an overview of the design, simulation, fabrication and analysis of a set of cladding modulated SWG Bragg gratings. First, an introduction to Bragg grating theory was laid out, followed by an overview of various silicon photonic Bragg grating geometries. The Bragg geometry focused upon in this thesis work was a cladding modulated grating with either a wire or SWG core, in which the gap of the silicon loading segments was modulated with a variety of cosine terms. A rigorous analysis of this system involved single unit-cell FDTD simulations to establish a relationship between $g$ and $\kappa$, which was then used to predict the resulting reflectance spectra via numerical FFT. This analysis was reinforced by performing FDTD simulations of an entire grating to establish the impact of cosine amplitude and period on sidelobe behaviour. Furthermore, thermal modulation was also studied as a means of inducing sidelobes. FEM simulations were performed using a periodic resistive heater, leading to a periodic temperature profile that induced sidelobes proportional in size to the supplied voltage. These devices were subsequently fabricated on a $220 \mathrm{~nm}$ SOI process and had their transmission spectra measured using edge couplers. The passive filters were highly successful, aligning quite well with the previous simulation results, whereas the thermal did not successfully induce sidelobes. With the exception of the fabrication and testing, all work presented in this chapter was my own. 


\section{Chapter 4}

\section{SWG Engineered Multimode Waveguide Bends}

This chapter contains an overview of the design, fabrication, and testing of a set of a compact multimode bend design for both the $220 \mathrm{~nm}$ and $340 \mathrm{~nm}$ SOI platforms. The bends use trapezoidal SWG segments and radial bridging strips to shape an index gradient, allowing for multiple mode orders to propagate with minimal loss and distortion. All design, simulation, layout, and analysis are were performed by myself. Fabrication was handled externally by nanophotonic foundry Applied Nanotools, and device measurements were performed by Shurui Wang at the National Research Council facility in Ottawa, Ontario.

\subsection{Bend Theory}

Waveguide bends are an integral building block of any photonic integrated circuit (PIC), serving as a routing component to maximize the integration density of the PIC. Smaller radius bends are desirable when designing PICs as they decrease the total footprint, but come at the expense of increased propagation loss, and have historically been limited to only the fundamental mode. However with proper modelling and compensation techniques photonic bends can not only be made smaller, but expanded to support higher order modes, vastly increasing a PIC's signal capacity. 


\subsubsection{Conformal Transformation of a Bent Waveguide}

Any curved optical waveguide can be modelled by applying the well-known conformal transformation demonstrated in [34]. It is derived by assuming the index profile of a standard circular bend as shown in Fig. 4.1 a), where $r_{1}$ and $r_{2}$ are the radii of its inner and outer edges, respectively. The index profile is defined explicitly by equation (4.1).

$$
n_{e f f}(\rho)= \begin{cases}n_{c l} & \rho<r_{1}, \quad \rho>r_{2} \\ n_{c o} & r_{1} \leq \rho \leq r_{2}\end{cases}
$$

By expressing the Cartesian coordinate space in terms of the complex plane $Z=x+i y$, a coordinate transformation can be applied in $W$ space in terms of $u$ and $v$. Proper selection of the function $f(Z)$ results in a straight waveguide in $W$ space. The required function is shown in (4.2).

$$
W=u+i v=f(Z)=f(x+i y)=r_{2} \ln \left(Z / r_{2}\right)
$$

Applying this conformal transformation gives the effective index gradient described by equation (4.3), which is plotted in Fig. 4.1 b). From this result we can infer a few critical points: i) Waveguide bends induce an index gradient that pushes modal fields to the outer edge of the bend. ii) The slope or sharpness of the index gradient is worsened for smaller radii. iii) For extremely sharp bends the effective cladding index on the outer edge may be greater than that of the core, leading to radiation leakage out of the bend.

$$
n_{\text {con }}(u)=n_{\text {eff }}(\rho(u)) e^{u / r_{2}}=n_{\text {eff }}\left(r_{2} e^{u / r_{2}}\right) e^{u / r_{2}}
$$


a)
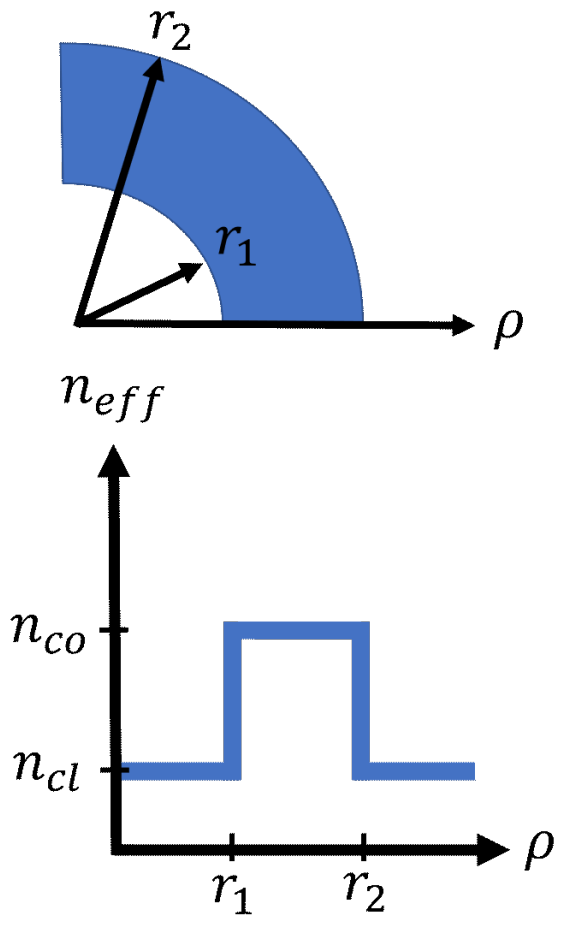

b)
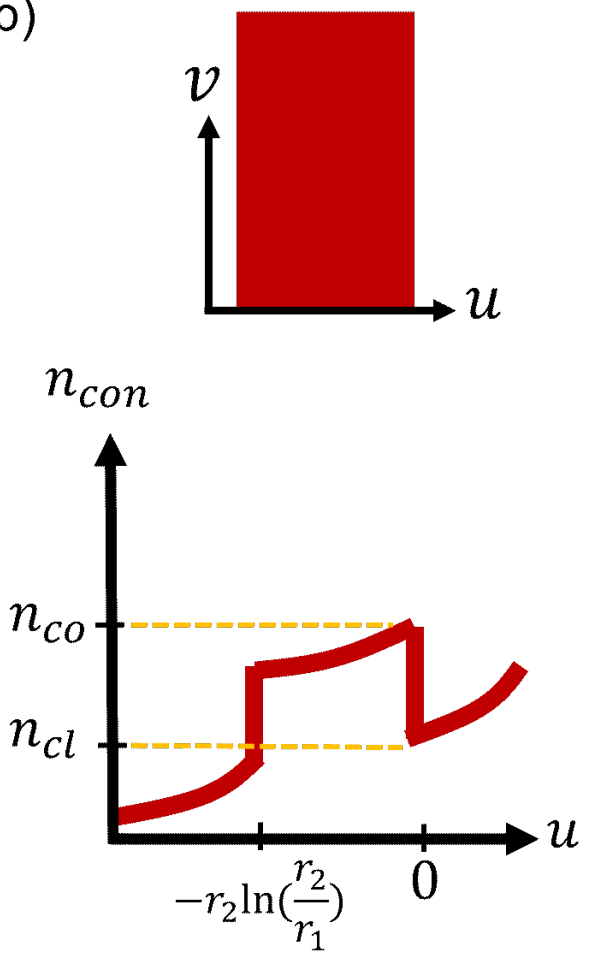

Figure 4.1: a) Index profile of a bent waveguide in $Z$ space. b) Conformal transformation of a bent waveguide index profile such that it is a straight waveguide in $W$ space.

High order modes are particularly susceptible to this index gradient. Whereas the fundamental mode may simply become delocalized towards the outer edge of the waveguide bend, a higher order mode will transfer power between their field maxima, producing a mode profile that is highly asymmetric. This asymmetry deforms the modal field to such an extreme degree that the output has a large difference in $n_{\text {eff }}$ value when compared to the input, rendering it useless for the purposes of designing an MDM system. For a $500 \mathrm{~nm}$ wide waveguide the fundamental mode can propagate unassisted in a circular bend with radii as small as $5 \mu \mathrm{m}$. This is not possible for multimode waveguides, where a waveguide of width $4 \mu \mathrm{m}$ that supports several modes would require a radius on the order of 
millimeters. Because of this fact much of the early research on SOI bends focused on single mode applications, but recent advances have shown great improvements in multimode bends, which will be discussed in section 4.2 .

\subsubsection{Sources of Bend Loss}

Compared to a straight waveguide with an equivalent cross-sectional geometry, bends suffer from additional sources of propagation loss. Material absorption in the C-band is negligible in SOI systems, leaving mode mismatch, radiation loss and sidewall scattering as the dominant loss mechanisms. Thorough investigations into the bend loss of SOI systems $[35,36]$ have shown a clear dependence for bend loss with respect to radius. In particular there are three distinct loss regions with respect to radius (small, intermediate and large) that are detailed in Fig. 4.2.
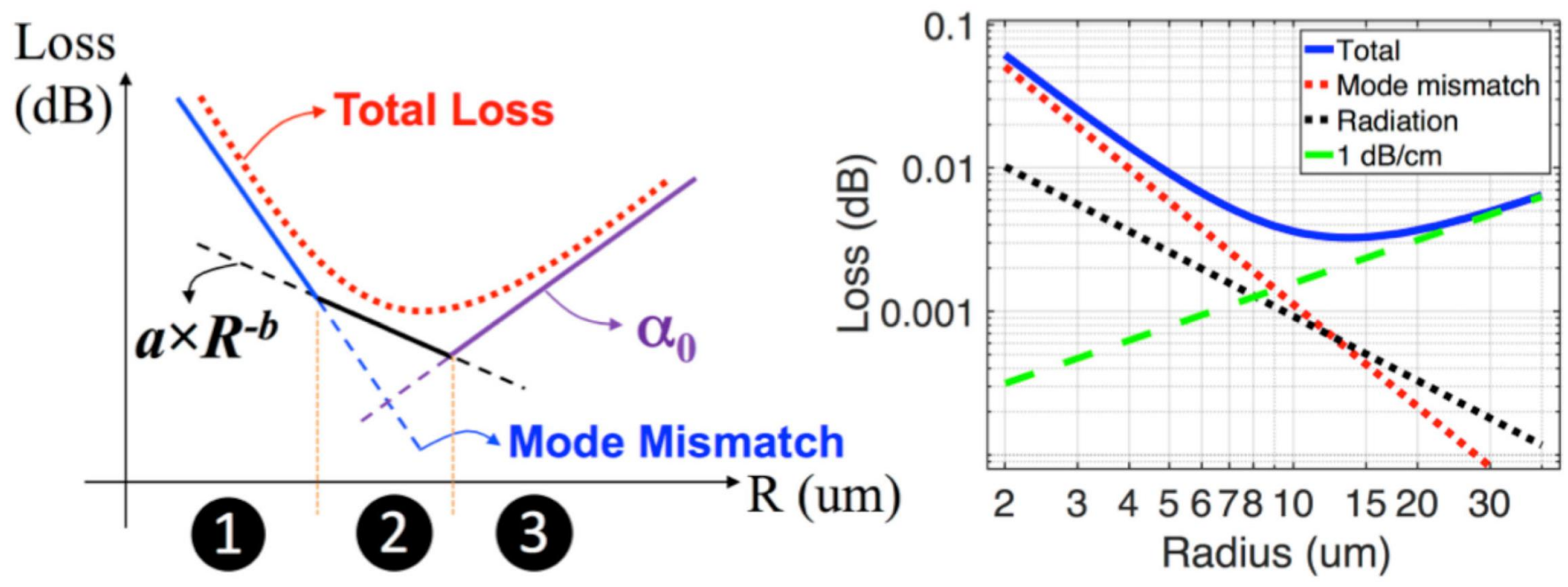

Figure 4.2: Bend loss quantified in terms of three regions based on the size of the radius, as defined in [35] @2019 IEEE. 


\section{Mode Mismatch}

Mode mismatch is the dominant source of loss for the majority of bends. It arises due to the mismatch between the index profile of a straight waveguide and that of a bend due to the previously discussed conformal transformation. Mode mismatch loss is at its worst when a straight waveguide transitions abruptly to a curved waveguide, such as in a conventional circular bend. This loss follows an inverse exponential relation with respect to radius, with small radii increasing it drastically. Mismatch loss can be mitigated by slowly varying the curvature from zero up to the maximum value, as in gradient curvature bends such as Bezier or Euler curves.

\section{Radiation Loss}

Radiation loss is present in straight waveguides to varying extents. For loosely confined modes (i.e. those close to the cutoff condition) this is quantized in terms of the imaginary part of the propagation constant. Lossy modes radiate power out into the cladding or substrate. This effect is only worsened in a bend, where the tilted index gradient shifts the modal field to the outer edge of the waveguide and power radiates out of the bend at the point where the cladding index is greater than the core index (see Fig. 4.3). 
a)

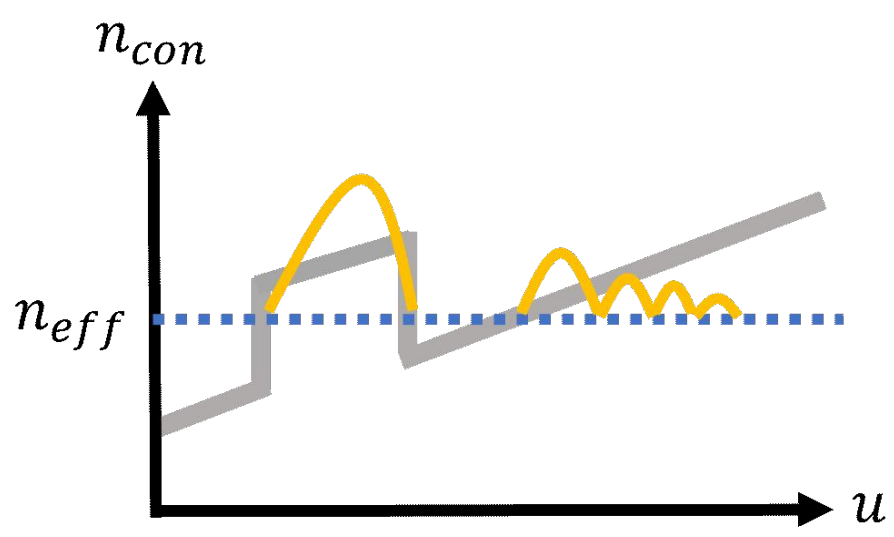

b)

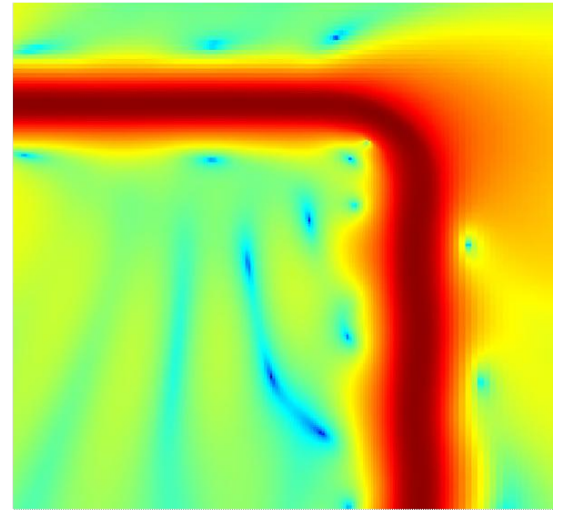

Figure 4.3: a) Graphical demonstration of radiation leakage into the cladding due to the gradient index of a bend. In yellow is the modal field of the fundamental mode, supported only in regions where the local index is greater than $n_{\text {eff }}$. b) Electric field simulation of a 1 $\mu m$ radius bend on a logarithmic scale, showing extreme leakage outwards into the cladding.

\section{Sidewall Scattering}

Sidewall scattering arises due to roughness in the shapes vertically etched by lithography processes [36]. The degree of scattering depends on the specifics of a given fabrication process, as well as the width of the waveguide. A mode with a high field concentration along its sidewalls will have a higher degree of scattering. Sidewall scattering is generally the dominant source of propagation loss in straight waveguides, but bent waveguides also suffer from it. For SOI waveguides sidewall scattering is typically a constant value between 1-3 $\mathrm{dB} / \mathrm{cm}$. This value is small compared to mode mismatch and radiation loss for smallto-intermediate radii, and only becomes dominant at radii greater than about $20 \mu \mathrm{m}$. This corresponds to region 3 in Fig. 4.2. 


\subsection{Literature Review of Multimode Bends in SOI}

There have been many attempts at solutions for implementing multimode bends in planar SOI waveguides. The solutions can be organized into 4 categories: i) Mode-converter assisted bends, ii) total-internal-reflection via corner bends, iii) gradient curvature bends, and iv) Gradient index bends.

\subsubsection{Mode-Converter Assisting Regions}

Mode-converter (MC) assisted bends are generally optimized for a small number of modes of a single polarization (often $\mathrm{TE}_{0} / \mathrm{TE}_{1}$ ). They consist of regular input/output waveguides of a fixed width that are connected to a bend via a MC section that is either optimized or inversely designed such that the two modes exit the bend with the same mode profile they entered with. Examples of this method include a segment of variable width [37], or a Y-branch geometry where one mode travels down each branch before recombining [38]. Nanohole engineering is a popular technique that uses inverse design to inscribe a grid of nanoholes in a square slab to produce multimode waveguide crossings, splitters, and $90^{\circ}$ bends. A two-mode bend consists of a large square slab of silicon filled with nanoholes such that the two mode orders travel through different paths before recombining, effectively creating a Y-branch [39]. The highest capacity example of the MC approach is a $30 \mu m$ radius $180^{\circ}$ bend that uses SWG nano-slot regions partially-etched into a PMMA polymer cladding [40]. It can support the first four TM modes, but requires a partial-etch and special cladding, in addition to the $\mathrm{MC}$ regions which increase the device length by $15 \mu \mathrm{m}$. A feature characteristic of MC-assisted bends is that the field distributions in the bends do not match up with those at the input/output. Whereas the input/output mode profiles have a clear 
number of maximums corresponding to the mode order, the field distribution in the bend spreads out and comes out of synch, and an optimal design is that which happens to result in the spatial mode returning to normal at the output. A sample of $\mathrm{MC}$ assisted bends are depicted in Fig. 4.4.

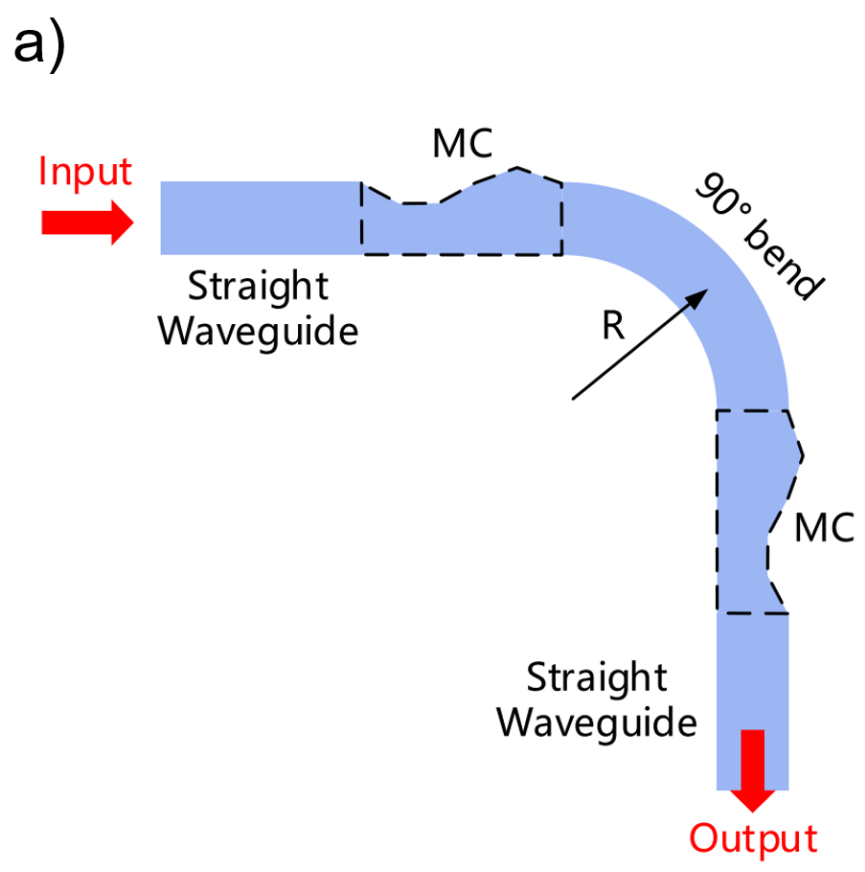

b)

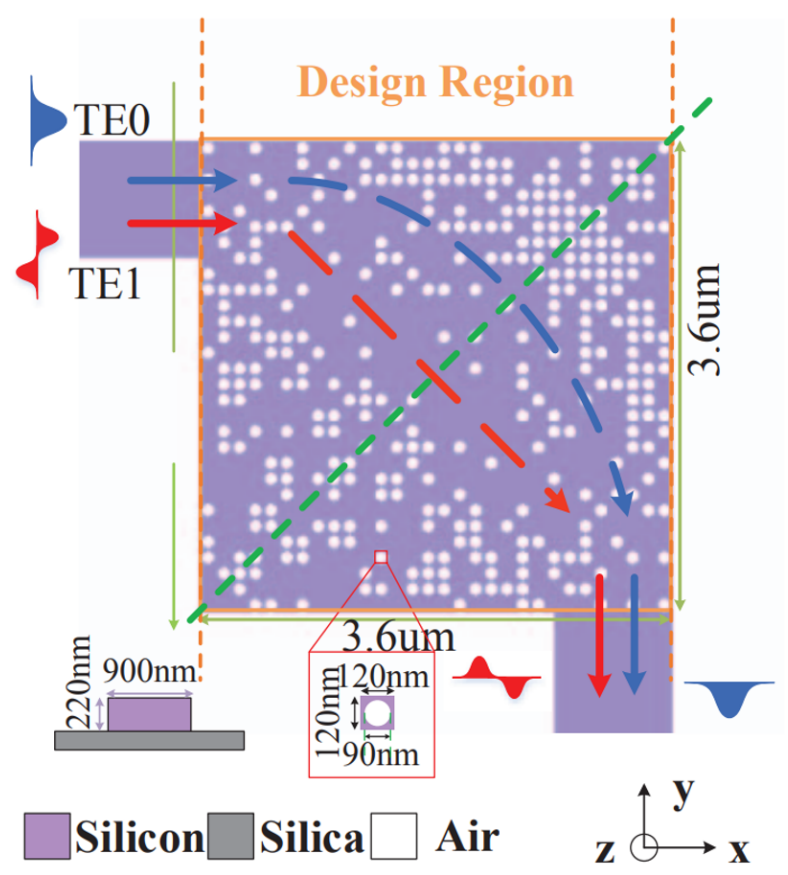

Figure 4.4: MC-assisted multimode bends: a) Variable width deformation, reprinted with permission from [37] () The Optical Society. b) Nanohole engineering, reprinted with permission from [39] () The Optical Society.

\subsubsection{Total-Internal-Reflection via Corner Bends}

Total-internal-reflection (TIR) via corner bends are a successful solution that utilizes a $45^{\circ}$ facet to perfectly reflect incoming light at a $90^{\circ}$ angle [41]. This technique is easily scaled up to higher order modes by increasing the width of the waveguide. The main downside is that the required widths to satisfy the TIR condition are significantly larger than the widths 
used in most multimode circuits, resulting in a solution with an excessively large footprint. An example of a TIR corner bend is shown in Fig. 4.5.
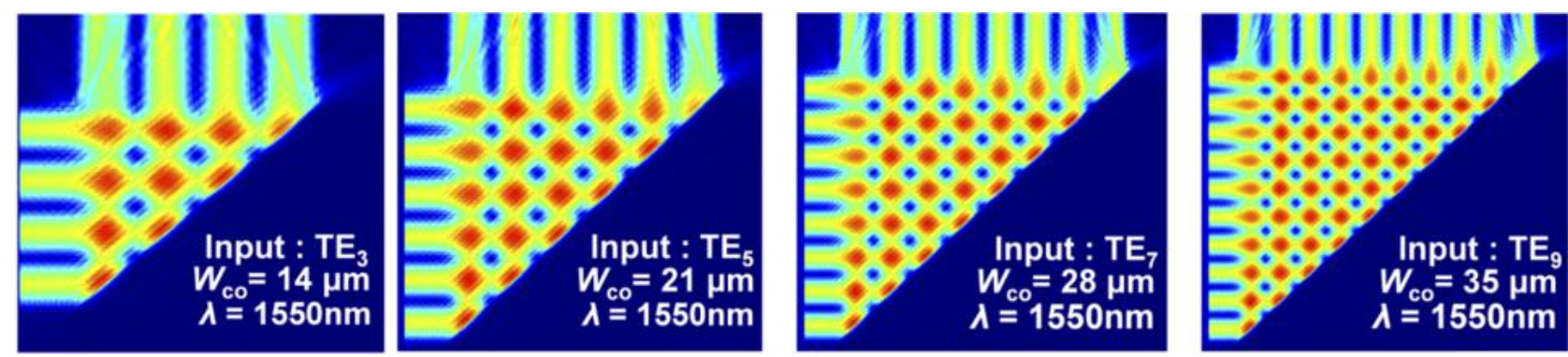

Figure 4.5: A multimode corner bend that uses total-internal-reflection. Reprinted with permission from [41] (C) The Optical Society.

\subsubsection{Gradient Curvature Bends}

Gradient curvature bends have been applied in both single and multimode systems with great success. Standard circular bends have a constant curvature along its path, defined by its constant radius. The main sources of loss in a SOI bend are from mode-mismatch at the input/output facets due to the mismatch in curvature, and radiation loss along the bend. Both of these loss factors increase exponentially as the radius decreases. These effects can be mitigated by using an alternate choice of geometric curve. Common choices include Bezier curves [42], Euler curves [43-45], and hybrid Euler-Circular curves [46]. A choice of custom bend has even been combined with a varying width when designing single mode bends as a means of reducing radiation loss by increasing the modal confinement at the point of sharpest curvature [47].

However the optimal choice of bend was investigated in [35] by developing a differential equation that minimizes the optical loss in the SOI platform. This differential equation factors in mode-mismatch, radiation loss, and sidewall roughness to arrive at its optimal 
$90^{\circ}$ bend shape. It has an extremely low curvature at the input/output facets and slightly higher curvature at the $45^{\circ}$ mid-point when compared to a circular bend. This slowly varying curvature is preferable to an abrupt change in curvature when designing multimode bends as the radial asymmetry is introduced much more slowly, and can therefore be compensated with gradient index tuning. This bend is the one adopted in this thesis work, and will be expanded upon later in section 4.4.

\subsubsection{Gradient Index Bends}

The most successful method of multimode bend design is the gradient index bend, where the radial asymmetry of the index profile is compensated by means of on-chip transformation optics. Early attempts at this approach employed a custom grayscale lithography process [48], varying the height of a $500 \mathrm{~nm}$ thick silicon layer such that the inner edge was taller than the outer edge. Although successful, this approach is severely limiting due to the custom fabrication process utilized, and has largely been superseded by SWG segment engineering.

This latter method uses trapezoidal SWG segments such that the duty-cycle is higher on one side of the segment than the other, resulting in a lateral index gradient. Bends that use conventional rectangular SWG segments tend to suffer from high radiation loss due to the low confinement of the mode profile, but trapezoidal SWG segments can compensate for this by having a larger duty-cycle on the inner radius and a smaller duty-cycle on the outer radius [49]. This technique has been widely used for designing single mode SWG bends with a full etch, but it can easily be scaled to multimode bend applications by partially etching the SWG segments on top of a slab waveguide. This approach has been used for circular bends [50], but is easily combined with gradient curvature bends for even better results [45]. This solution is by far the most effective because it supports the first 3 modes for both 
polarizations with an effective radius of $10 \mu \mathrm{m}$. One elegant characteristic of the gradient index solution is that the field distribution within the bend remains co-centric along the path of curvature, unlike the MC-assisted bends. The maximums and minimums of the mode field profiles remain obvious for the entire bend radius, making it possible to visually assess the strength of the index gradient. If power is significantly transferred between the maximums of the field profile then the index gradient is either too extreme or too mild. A sample of gradient curvature and gradient index bends are depicted in Fig. 4.6.

a) $90^{\circ}$ Euler-bend

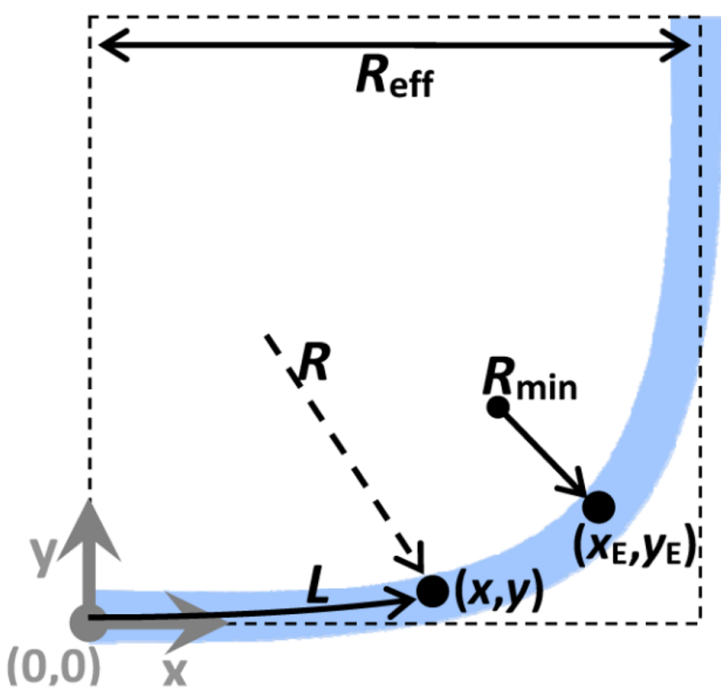

b)

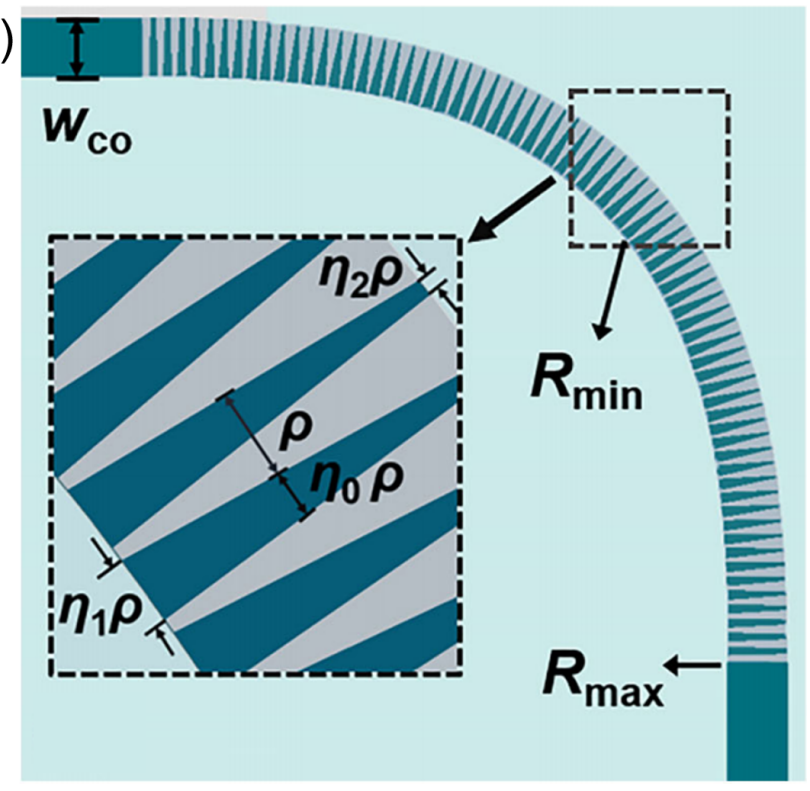

Figure 4.6: A sample of multimode bends that use gradient curvature and gradient index engineering: a) A Euler bend, reprinted with permission from [43] (C) The Optical Society. b) Partially-etched SWG segments [45] (O2020 IEEE.

\subsection{Proposed Design}

My proposed solution combines gradient curvature bends with gradient index bends to increase the number of usable modes, while also maintaining a small radius. Additionally, 
we plan to implement this solution with just a single etch step. Prior to this work multimode bends have only implemented the index gradient in a shallow partially-etched layer, and we propose implementing the index gradient throughout the entire silicon device layer. To achieve this we combine trapezoidal SWG strips alongside radially bridging-strips, pictured in Fig. 4.7. These bridging-strips will vary their width as a function of the bend curvature, with the outer strips decreasing in width, and the inner strips increasing in width, which will provide an additional degree of freedom over the index gradient. This is in addition to the trapezoidal SWG segments, whose duty-cycle ratio is limited by fabrication considerations. By etching the full device layer this allows for a sharper index gradient, and thus a wider core, which can support more modes. A full etch has the added advantage of no vertical asymmetry, which leads to reduced scattering of TM polarized modes. 


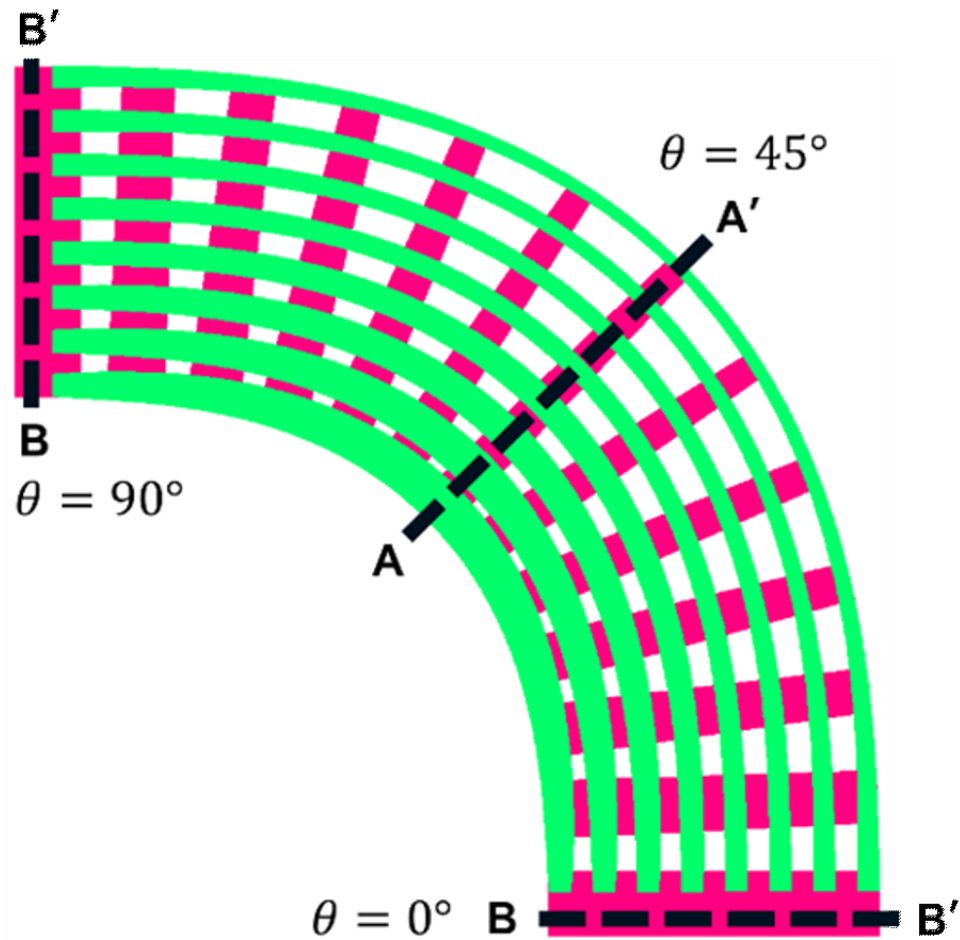

Figure 4.7: A schematic of the multimode bend geometry illustrating the changing dimensions of the trapezoidal segments (red) and the adiabatically tapered radial strips (green) from $\theta=0^{\circ}$ to $\theta=90^{\circ}$. The point of maximum curvature is along line AA' at $\theta=45^{\circ}$.

The parallel SWG strips increase the relative effective index of the bend compared to a bend that uses only trapezoidal SWG segments. This means there is a smaller index mismatch at the input and output facets, compensating for the fully-etched design. To reduce the excess-losses we further propose a taper to be implemented at the input and output of the bend below $5 \mu \mathrm{m}$ in size. To the best of our knowledge there has not been a fully etched SWG solution to multimode bends. 


\subsection{Choice of Bend Curve}

As briefly discussed in section 4.2.3 there are many choices of waveguide bend that offer performance advantages over that of a standard circle. A brief discussion of possible bend alternatives is presented before introducing the optimal bend derived in [35], which is the one used in this thesis work.

\subsubsection{Common Bend Alternatives}

A circular bend is the most common bend implemented in photonic circuits due to its simple geometry. It has a constant curvature which results in a large index mismatch at the input facets. A Bezier bend is defined by four control points: $(0,0),(R, R),(R(1-m), 0),(R, m)$. It has a variable curvature, and for minimal bend loss the ideal value of $m$ is approximately 0.291 [42]. Although the curvature at the facet is much lower than a circular bend, it is still non-zero. The Euler bend improves upon the Bezier curve as its curvature varies linearly along the bend, with zero curvature at its input facets [44]. A hybrid Euler-circular begins and ends with Euler curve segments, but the middle section is replaced with a circular bend segment [46]. This maintains the zero curvature at the input facts while also reducing the maximum curvature. Although each of these curves provide improvements over the standard circular bend, they are outperformed by the optimized bend in the region of interest, i.e. radii between $4-10 \mu m[35]$.

\subsubsection{Optimal Bend}

The optimal $90^{\circ}$ bend is an empirically derived curve studied in [35]. The work establishes a differential equation in terms of minimizing bend loss in an SOI platform. It defines the 
total loss in Eq. (4.4) in terms of radiation loss $\left(\alpha_{r}\right)$, sidewall scattering $\left(\alpha_{s w}\right)$ and mode mismatch $\left(\alpha_{m}\right)$, all of which are functions of the radius.

$$
\alpha_{d B / c m}[R] \approx \alpha_{r}[R]+\alpha_{s w}[R]+\alpha_{m}[R]
$$

A boundary condition is applied at the input and output such that the curvature is zero, therefore setting $\alpha_{m}$ equal to zero. Under this boundary condition sidewall roughness and radiation loss can be approximated via Eq. (4.5), where sidewall roughness loss is a constant independent of radius $\left(\alpha_{0}\right)$, and radiation loss is defined empirically via $a R^{-b}$, with $a=$ $181.98 \mathrm{~dB} / \mathrm{cm}$ and $b=2.49$. By enforcing the zero curvature boundary condition at the input and output the optimized curve can be calculated for each of the three loss regions. The differential equation that defines this curve is shown in more detail in section 4.7.1.

$$
\alpha_{r}[R]+\alpha_{s w}[R]=a \times\left(R_{\mu m}\right)^{-b}+\alpha_{0}
$$

For large radii (upwards of $20 \mu \mathrm{m})$ the constant radiation loss is dominant $\left(\alpha_{r}, 1 \mathrm{~dB} / \mathrm{cm}\right)$, and a circular bend is the solution to the differential equation. For small radii (below $8 \mu \mathrm{m}$ ) mode mismatch at the input and output facets is the dominant loss mechanism. The optimal bend is defined such that the curvature at the input and output approach that of a straight waveguide, leaving the $a R^{-b}$ radiation term as the dominant loss term. The intermediate region is influenced primarily by the mode mismatch, and possibly the radiation loss. The optimal bend is quite similar to the small radii case with minimal adjustments, with the $a R^{-b}$ term being smaller due to the larger radii. A radius of $10 \mu m$ was chosen so that the bend falls well within the intermediate region, meaning the optimal bend equation applies. 


\subsection{Waveguide Geometry Cross-section}

The height of the Silicon device layer must be determined first before selecting any other dimension for the bend. The height has a large impact on the effective indices of all modes, but especially the TM modes. SOI device layer thicknesses come in only a few select sizes due to fabrication constraints. Two of the most standard thicknesses, $340 \mathrm{~nm}$ and $220 \mathrm{~nm}$, were chosen for this analysis due to their wide availability. The $340 \mathrm{~nm}$ case was the main focus of this work as its large height allows it to support an equal amount of TM modes as TE, whereas the $220 \mathrm{~nm}$ focused entirely on TE modes.

The selection of the width needs to consider two factors: i) How many modes it can support, and ii) The radial asymmetry. A larger width increases the number of mode orders than may propagate in a given structure, as well as increasing the $n_{e f f}$ values of all modes. On the other hand a larger width increases the asymmetry due to a bend, with higher mode delocalization for a given radius. The width needs to be high enough to support the desired number of modes, but not so large as to introduce excessive radial asymmetry. Two different widths may be able to support the same number of modes, but the lower width will be more susceptible to radiation bend-loss, and the higher width will need a sharper index gradient to compensate the increased asymmetry.

\subsubsection{Thickness of $340 \mathrm{~nm}$}

The width of the waveguide was selected through a parameter sweep, with the goal of supporting 5 TE and 5 TM modes. The resulting dispersion curve can be seen in Fig. 4.8, where the curves are plotted in terms of the 10 largest $n_{\text {eff }}$ values. At widths greater than $2.06 \mu \mathrm{m}$ the first 10 modes consist of $5 \mathrm{TE}$ and $5 \mathrm{TM}$ modes, meaning the chosen 
width should be larger than $2.06 \mu \mathrm{m}$, while minimizing any potential intermodal crosstalk. Intermodal crosstalk arises when two modes have similar effective indices leading to power coupling between them. For rectangular waveguides this most frequently occurs between the $\mathrm{TE}_{i}$ and the $\mathrm{TM}_{i-1}$ or $\mathrm{TM}_{i-2}$ modes, as the dispersion curves for low order $\mathrm{TM}$ modes tend to intersect higher order TE modes as the width is increased.

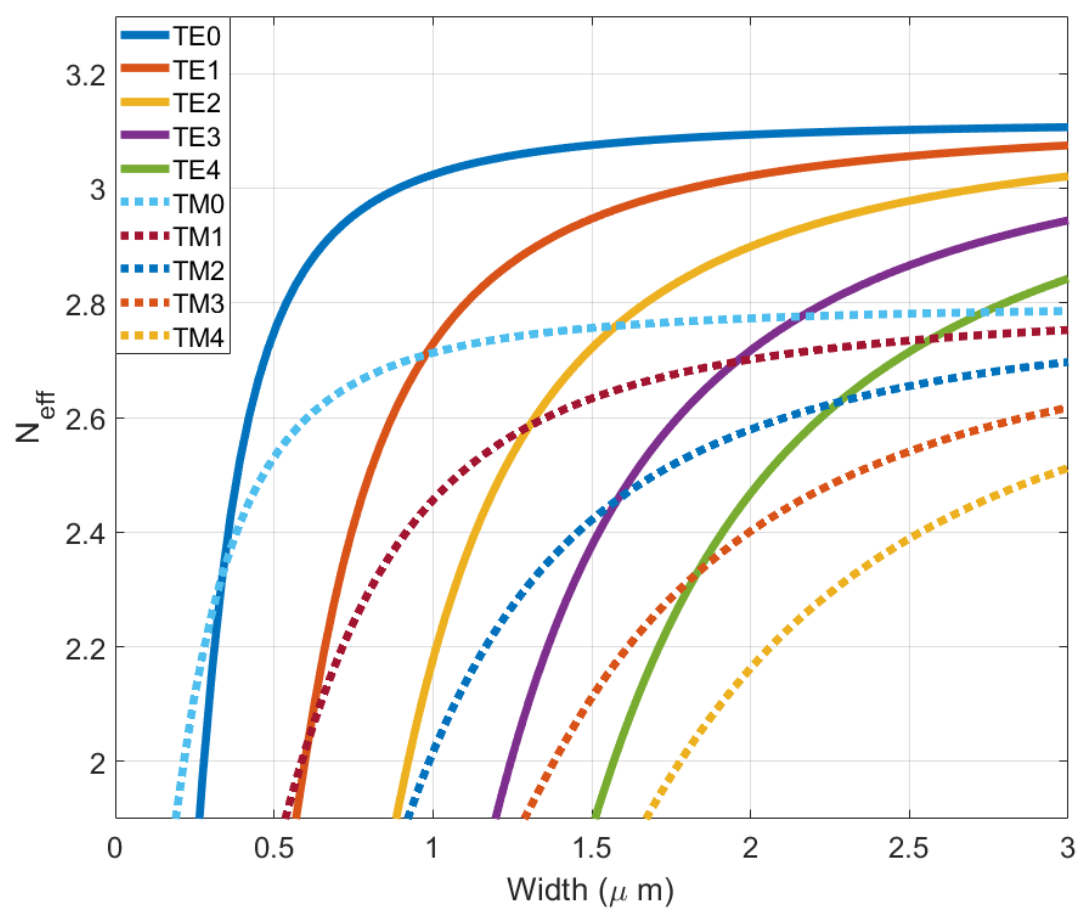

Figure 4.8: Dispersion sweep of a $340 \mathrm{~nm}$ tall silicon waveguide showing the first $5 \mathrm{TE}$ and first 5 TM modes.

As can be seen in Fig. 4.8 the dispersion curves become quite dense upwards of $1.5 \mu \mathrm{m}$, with only very narrow ranges where all curves are separated. In particular the spacing of the $\mathrm{TM}_{0}, \mathrm{TE}_{3}$ and $\mathrm{TM}_{1}$ modes is quite narrow, and a width of $2.075 \mu \mathrm{m}$ was selected to maximize the spacing, as seen in a zoomed-in view in Fig. 4.9. 


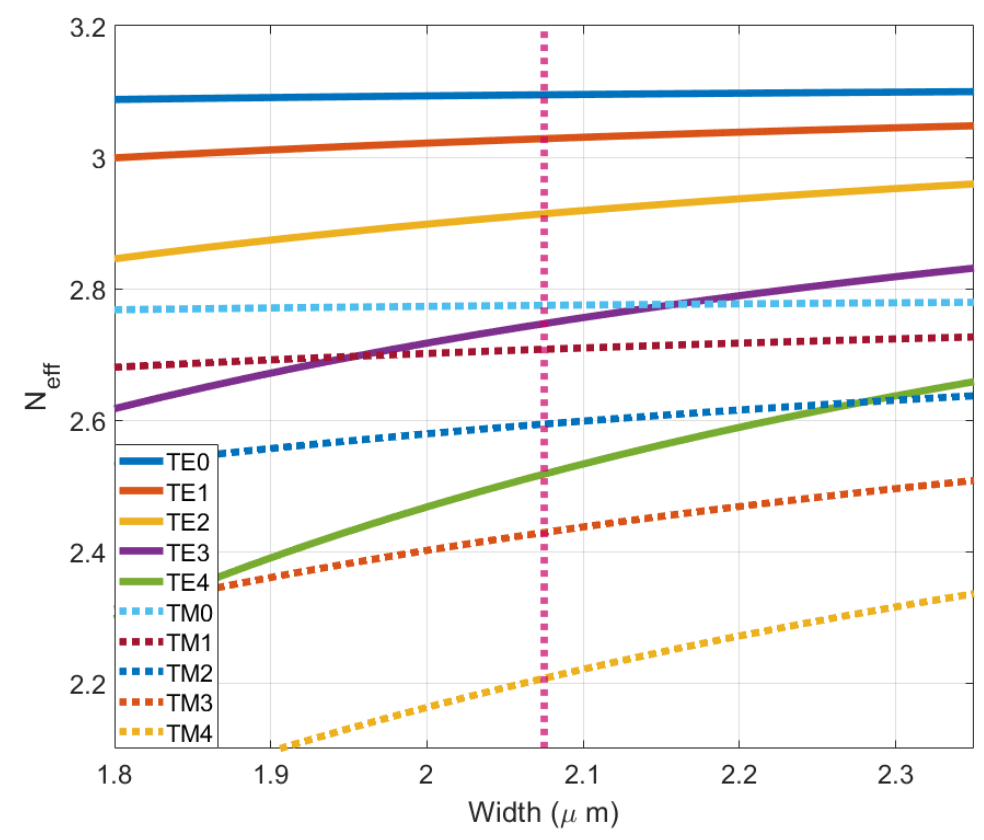

Figure 4.9: Dispersion sweep of a $340 \mathrm{~nm}$ tall silicon waveguide at the design width of $2.075 \mu \mathrm{m}$.

\subsubsection{Thickness of $220 \mathrm{~nm}$}

To expedite the optimization process the $220 \mathrm{~nm}$ design reused the same width as the 340 nm design, i.e. $2.075 \mu \mathrm{m}$. The goal of the $220 \mathrm{~nm}$ design was to support exclusively TE modes, ideally up to $\mathrm{TE}_{3}$. The dispersion sweep, as pictured in Fig. 4.10 shows that at a width of $2.075 \mu \mathrm{m}$ the first five modes are all $\mathrm{TE}$ and thus there is no risk of intermodal crosstalk with the TM modes. A zoomed-in view of this region of interest in shown in Fig. 4.11. As anticipated the TE modes all have lower effective indices when compared to their $340 \mathrm{~nm}$ equivalents. The effective indices of the TM modes are so low that much of their power will radiate out of the bend due to the sharp radius. 


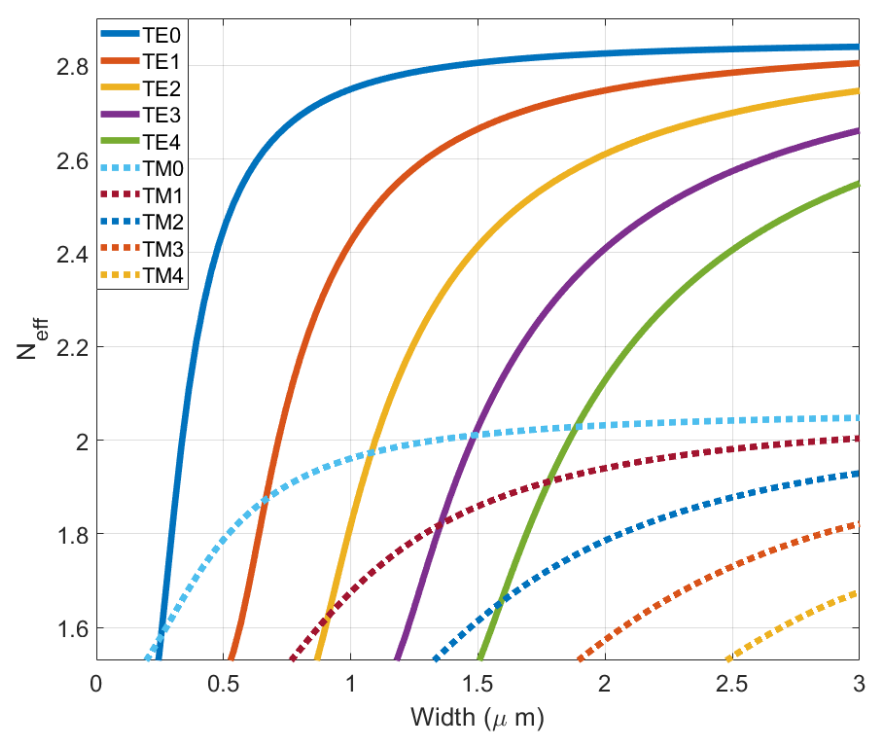

Figure 4.10: Dispersion sweep of a $220 \mathrm{~nm}$ tall silicon waveguide showing the first $5 \mathrm{TE}$ and first 5 TM modes.

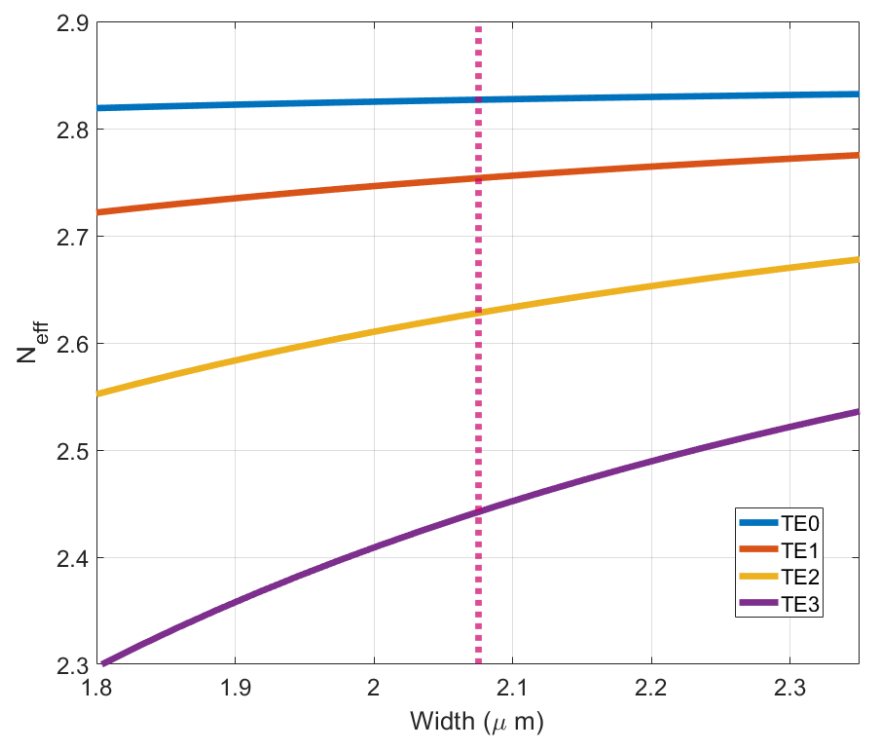

Figure 4.11: Dispersion sweep of a $220 \mathrm{~nm}$ tall silicon waveguide at the design width of $2.075 \mu \mathrm{m}$. 


\subsection{Mode Delocalization vs Radius}

As discussed in section 4.1.1, the index profile of a bent waveguide can be modelled as that of a straight waveguide with a conformal transformation applied to it. The index profile effectively has a gradient that pushes the modal field towards the outer radius of the bend. This delocalization of the modal field has an exponential dependence on the radius, with smaller radii worsening the asymmetry of the modal fields. This delocalization effect can be quantized in terms of the mode overlap, defined by Eq. (4.6). Mode overlap calculates the fractional power transfer between one mode profile to another, resulting in a single positive number between 0-1. By comparing the field of a straight waveguide $\left(E_{1} / H_{1}\right)$ with that of a bent waveguide $\left(E_{2} / H_{2}\right)$, mode overlap can be used to quantify the asymmetry induced by a given radius.

$$
\text { Overlap }=\left|\operatorname{Re}\left[\frac{\left(\int E_{1} \times H_{2}^{*} \cdot d S\right)\left(\int E_{2} \times H_{1}^{*} \cdot d S\right)}{\int E_{1} \times H_{1}^{*} \cdot d S}\right] \frac{1}{\operatorname{Re}\left(\int E_{2} \times H_{2}^{*} \cdot d S\right)}\right|
$$

Mode overlap was calculated for each of the first four TE modes in a waveguide of crosssection $2.075 \mu \mathrm{m}$ by $220 \mathrm{~nm}$ as a function of radius, and is plotted in Fig. 4.12. As expected all modes approach an overlap of $100 \%$ when the radius goes to infinity, and overlap decreases exponentially as the radius decreases. Additionally, an interesting pattern emerges between the mode orders when analyzing the region around the target radius of $10 \mu m$. For extremely small radii (below $5 \mu \mathrm{m}$ ) only the fundamental mode is supported, with the higher order modes being squeezed out of the waveguide core due to their low $n_{\text {eff }}$ values. However, at the target radius of $10 \mu \mathrm{m}$ the $\mathrm{TE}_{3}$ mode is shown to have the highest overlap, followed by $\mathrm{TE}_{0}$, $\mathrm{TE}_{2}$ and finally $\mathrm{TE}_{1}$. From observing the whole graph one can surmise that the fundamental mode has the best overlap for small radii, but higher order modes have a sharper slope which 
results in better overlap at intermediate-to-large radii.

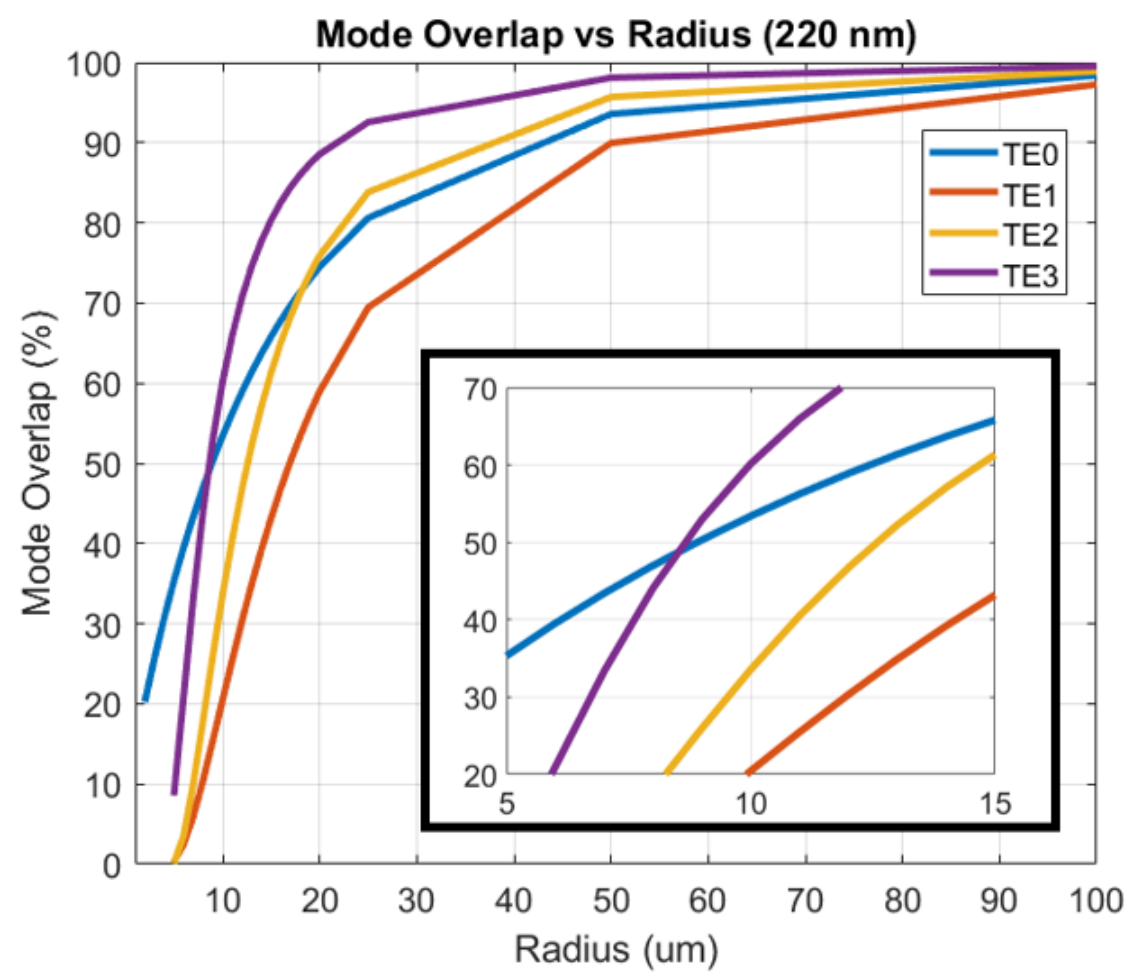

Figure 4.12: Mode overlap as a function of radius for a $2.075 \mu \mathrm{m}$ by $220 \mathrm{~nm}$ waveguide for the first four TE modes.

Similarly, the case of a waveguide of cross-section $2.075 \mu \mathrm{m}$ by $340 \mathrm{~nm}$, with both the TE and TM modes, is plotted in Fig. 4.13. The $340 \mathrm{~nm}$ case follows the same general trend, but with each respective mode order having an overall lower mode overlap when compared to its $220 \mathrm{~nm}$ equivalent. As previously discussed, this can thought of qualitatively as the modes having more room to delocalize, as they are less confined by the boundaries of the silicon core. For a given mode order the TE and TM overlap values differ only by 1-2\%, with the TM modes performing better at all radii. For all cross-sections and for all radii, overlap of $\mathrm{TE} / \mathrm{TM}_{i+1}>\mathrm{TE} / \mathrm{TM}_{i}(i \neq 0)$. 
4. SWG Engineered Multimode Waveguide Bends
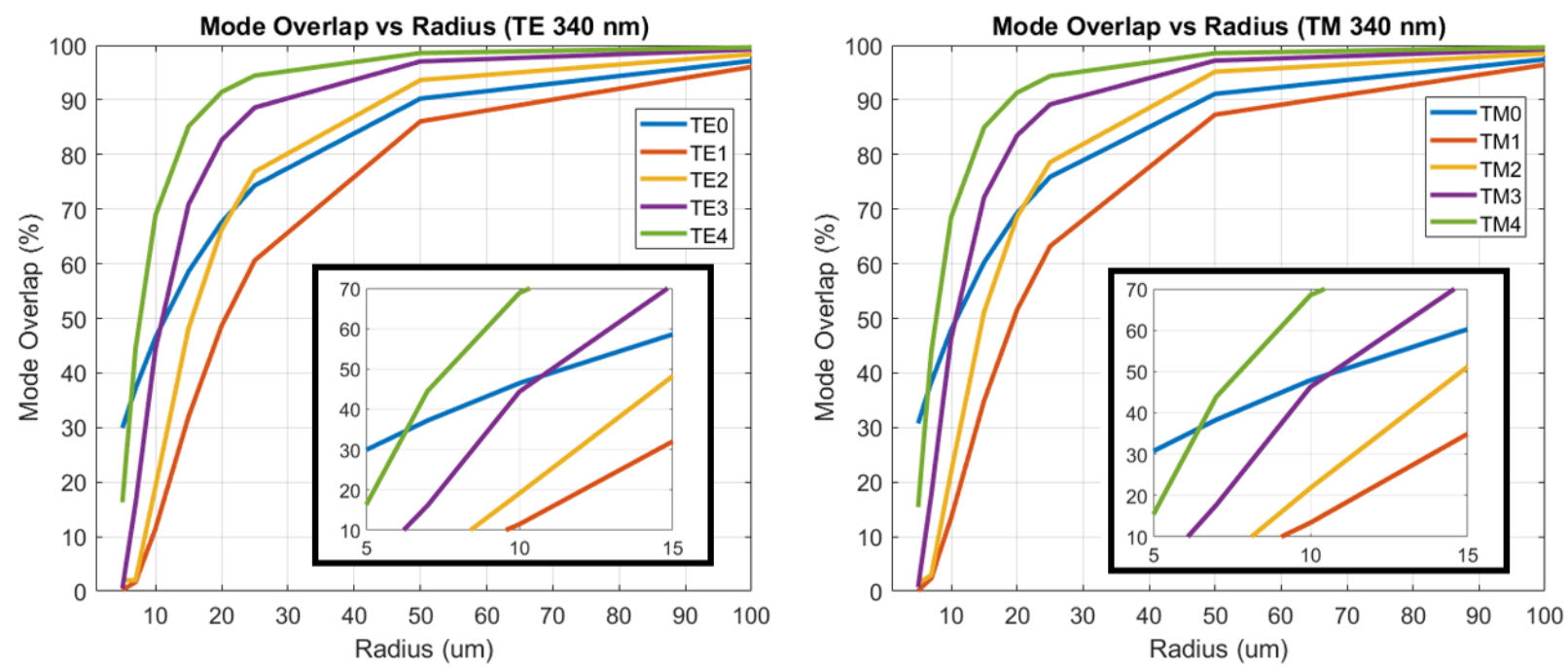

Figure 4.13: Mode overlap as a function of radius for a $2.075 \mu \mathrm{m}$ by $220 \mathrm{~nm}$ waveguide for the first five TE and TM modes.

The trend observed in these plots suggests that for the target radius of $10 \mu \mathrm{m}$ the fundamental mode and the highest order modes will be the easiest to compensate for, and the intermediate order modes will be the most difficult to compensate. For the $220 \mathrm{~nm}$ case the design focus should be the $\mathrm{TE}_{1}$ and $\mathrm{TE}_{2}$ modes, and for the $340 \mathrm{~nm}$ the focus should be on the $\mathrm{TE} / \mathrm{TM}_{1}$ and $\mathrm{TE} / \mathrm{TM}_{2}$. This hypothesis is supported by the optimization process in section 4.8.2, where these modes demonstrated the highest sensitivity to parameter sweeps.

\subsection{Index Gradient Engineering}

\subsubsection{Calculation of Optimized Curve}

The optimized curve for SOI bends was defined in [35] using a differential equation parameterized by $\theta$, the solution to which is shown explicitly in Eq. (4.7) and (4.8). $R_{\text {eff }}$ is the desired effective radius, $b$ is an empirical value equal to 2.49 , and the unknown 
parameters $x_{0}$ and $A$ can be found by solving the boundary condition at $\theta=\pi / 2$. The values of these parameters are shown in Eq. (4.9).

$$
\begin{gathered}
x=x_{0}+\frac{1}{A} \int_{\pi / 4}^{\theta}(\cos \theta)^{\frac{b-1}{b}} d \theta \\
y=R_{e f f}-x_{0}+\frac{1}{A} \int_{\pi / 4}^{\theta} \sin \theta(\cos \theta)^{\frac{-1}{b}} d \theta \\
x_{0}=R_{e f f} \frac{F_{2}}{F_{1}+F_{2}}, A=\frac{F_{1}+F_{2}}{R_{e f f}} \\
F_{1}=A\left(R_{e f f}-x_{0}\right)=\int_{\pi / 4}^{\pi / 2}(\cos \theta)^{\frac{b-1}{b}} d \theta \\
F_{2}=A x_{0}=\int_{\pi / 4}^{\pi / 2} \sin \theta(\cos \theta)^{\frac{-1}{b}} d \theta
\end{gathered}
$$

Calculating the optimal curve requires two input parameters: The desired effective radius and the desired SWG pitch (10 $\mu \mathrm{m}$ and $220 \mathrm{~nm}$ respectively). A large number of sample points (ex: $n_{\text {sample }}=500$ ) was used to define a linearly spaced vector of angle $\theta$ from $\pi / 4$ to $\pi / 2$. Only half of the $90^{\circ}$ span needs to be calculated as the remaining half can be extracted from symmetry across the $\theta=\pi / 4$ line. Using the equations discussed above, the vector of $\theta$ values was subbed-in and used to calculate a set $\mathrm{X} / \mathrm{Y}$ coordinate pairs. A calculation was run on these points to calculate the total arc length by summing the changes in $\mathrm{x}$ and $\mathrm{y}$, as in Eq. (4.10). Dividing the total arc length by the SWG pitch gives the number of SWG $\left(n_{S W G}\right)$ required to populate the curve. For half the effective radius of $10 \mu m$ the arc length was approximately $8.47 \mu \mathrm{m}$, which can fit 33 SWG segments at a pitch of $220 \mathrm{~nm}$.

$$
s=\int d s, \text { where } d s=\sqrt{(d x)^{2}+(d y)^{2}}
$$


The next step was to extract 33 points from the sample coordinate set such that the SWG coordinates are equidistant from each other (i.e. the data-set being reduced from 500 to 33 points). By default, a curve parameterized by $\theta$ has points more closely spaced in sections of high curvature, and spaced further apart in sections of low curvature. The spacing of the SWG segments must be a constant pitch so that the effective index of the bend remains uniform. Code was written that swept through all the sample points and identified 33 coordinate pairs that had an equidistant displacement of $220 \mathrm{~nm}$. Symmetry was then used to calculate the full $90^{\circ}$ span, resulting in a data set of 77 coordinate pairs. The resulting curve is depicted in Fig. 4.14.
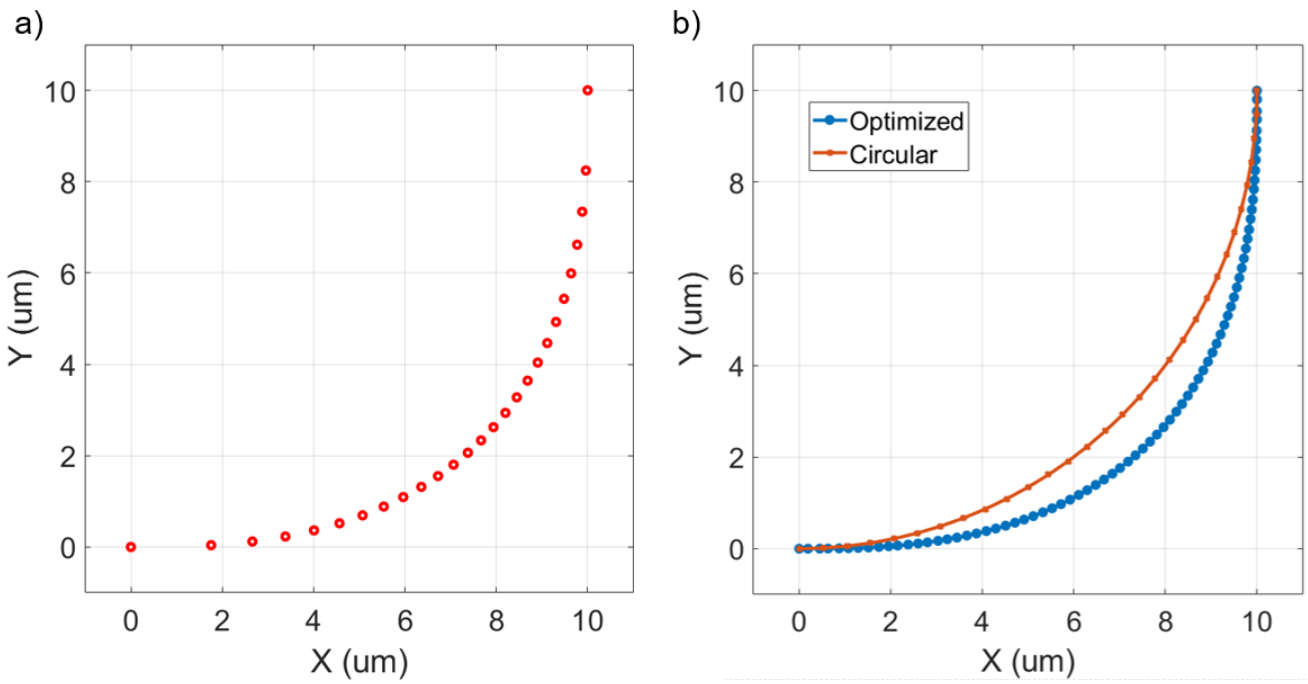

Figure 4.14: a) Optimized curve parameterized by $\theta$. b) The optimized curve with equidistant spacing of $220 \mathrm{~nm}$. A conventional circular bend is shown for reference.

The two remaining calculations were the to find the instantaneous curvature at each SWG coordinate pair, as well as the rotation angle with respect to the origin. Note that rotation angle is different from the parameter $\theta$, as $\theta$ varies linearly from $\pi / 4$ to $\pi / 2$, and the rotation angle varies as a function of curvature. These quantities will be used to set the 
duty-cycle of the trapezoidal SWG segments and their rotation angle, as well as the width of the bridged-SWG strips. The curvature and rotation angle are related to the instantaneous derivatives of $x$ and $y$ at each coordinate pair, as shown in Eq. (4.11) and (4.12).

$$
\begin{aligned}
& C=\frac{d \theta}{d s}=\cos \theta \frac{d \theta}{d x}=\sin \theta \frac{d \theta}{d y} \\
& \text { Rot. Angle }=\operatorname{atan} 2\left(\frac{d y}{d \theta}, \frac{d x}{d \theta}\right)
\end{aligned}
$$

The final data set consists of 4 by 77 array, where the 4 columns are the $x$ value, $y$ value, rotation angle, and curvature. These values are critical for generating the geometries that will be simulated in Lumerical FDTD. The curvature at each of these coordinates and the corresponding rotation angle are depicted in Fig. 4.15.

a)

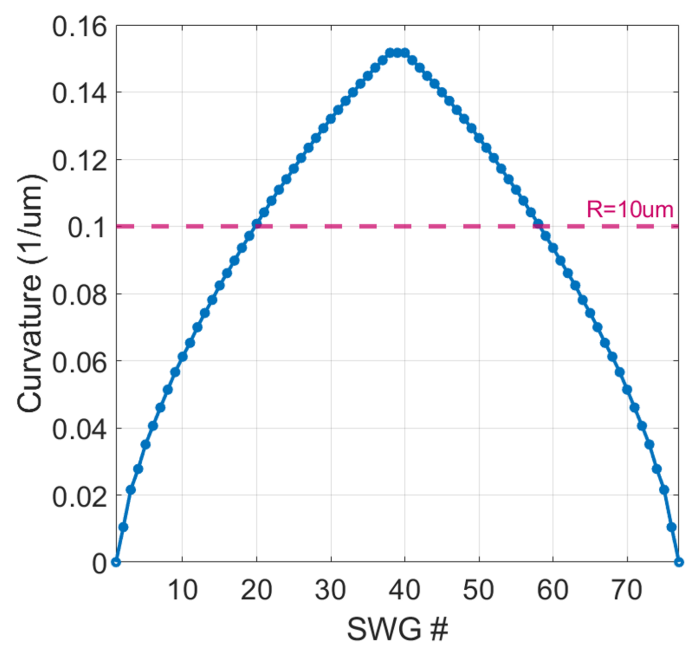

b)

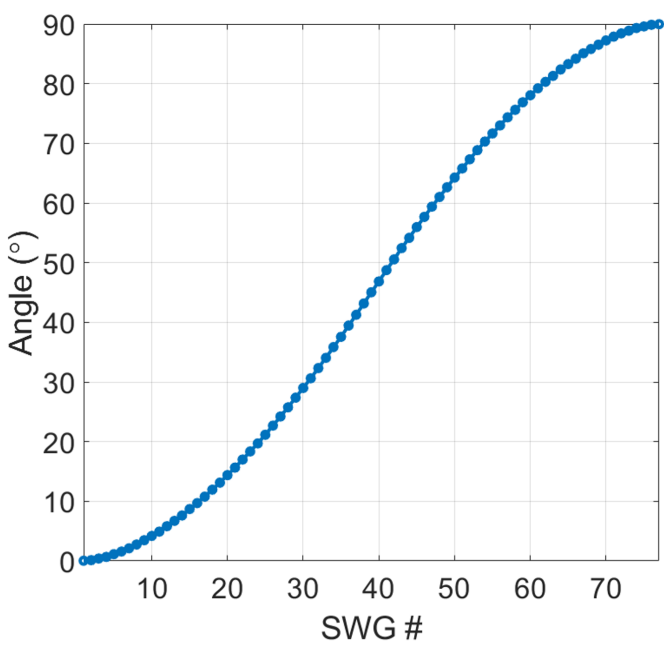

Figure 4.15: a) Curvature at each individual SWG segment. b) Rotation angle of each individual SWG segment. 


\subsubsection{Trapezoidal SWG Segments}

Trapezoidal SWG segments have been widely explored as technique for designing SWG bends, first with single mode applications [49] but more recently for multimode applications. The SWG segment is warped from its usual rectangular dimensions into a trapezoid, such that the inner radius of a bend has a longer side length than the outer radius (see Fig. 4.16). The effective result of this geometry is an index gradient that balances out the index gradient introduced by the bend. The index profile of the bend can effectively replicate that of a straight waveguide.

$$
\eta_{1}=\eta_{2}
$$

a)

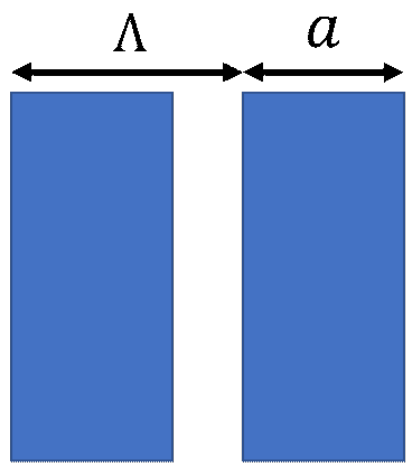

b)

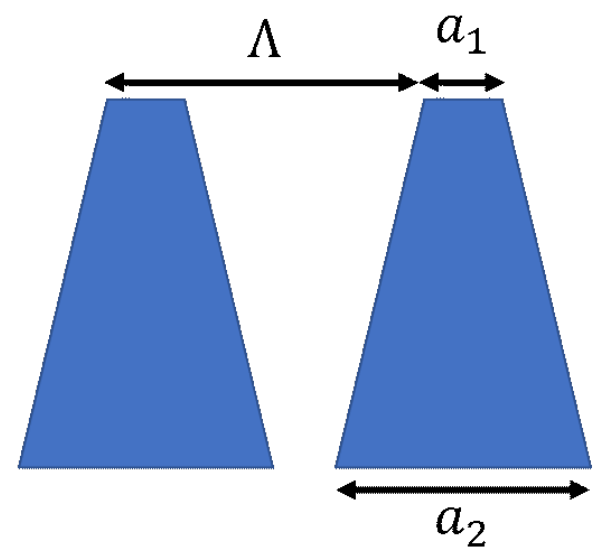

Figure 4.16: a) A standard rectangular SWG. b) A trapezoidal SWG with different dutycycles on each side.

Early instances of trapezoidal SWG segments were for circular bends, meaning the curvature was constant for the entire bend, and $\eta_{1} / \eta_{2}$ has a constant value. For a bend with a varying curvature the ratio of $\eta_{1} / \eta_{2}$ must vary along the bend to compensate for changing index profile. The optimized bend has zero curvature at the input and output 
facets, and thus begins with $\eta_{1} / \eta_{2}$ equal to 1 .

In order to comply with fabrication constraints both duty-cycles were set to $60 \%$. This value is large to ensure the higher order modes are not cutoff, while also leaving a gap of 88 $\mathrm{nm}$ between adjacent segments, comfortably above the minimum feature size of $60 \mathrm{~nm}$. By defining the ratio at the point of maximum curvature $\left(\theta=45^{\circ}\right)$, the ratio $\eta_{1} / \eta_{2}$ is calculated for all other SWG segments as a function of the curvature, as seen in Fig. 4.17

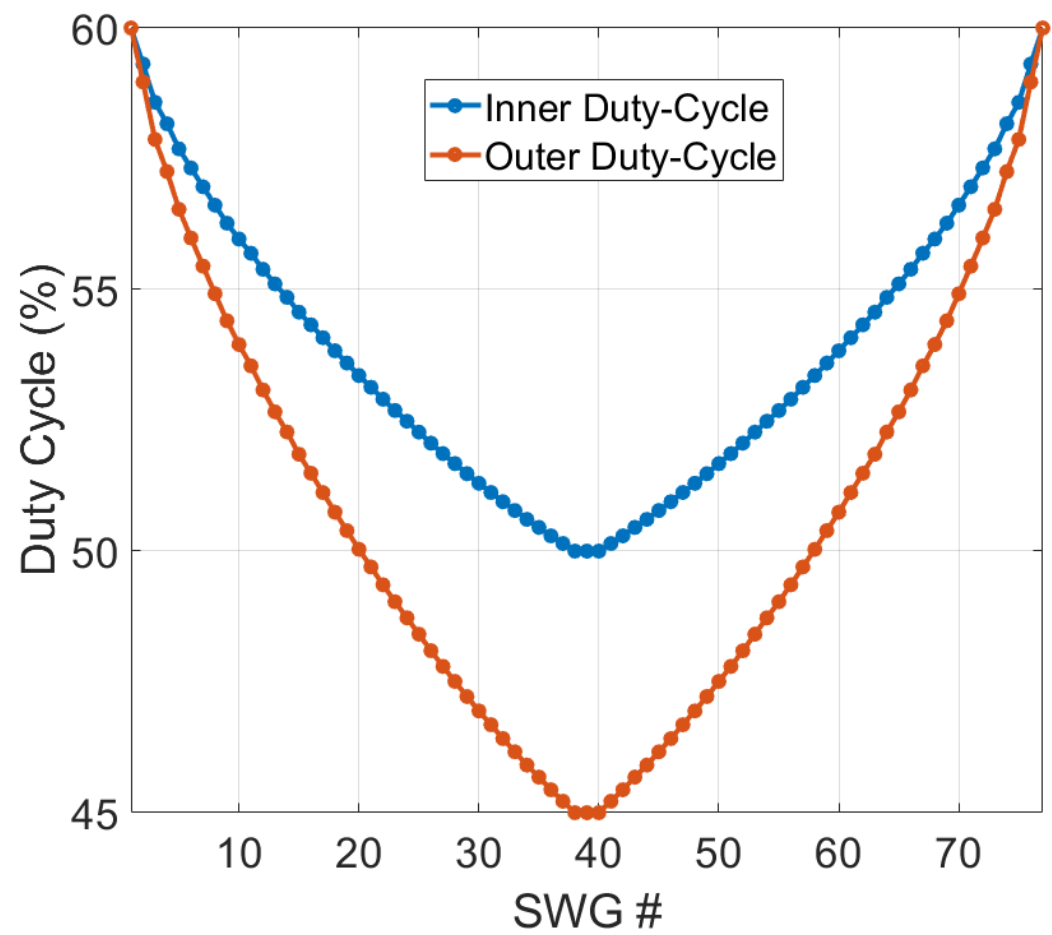

Figure 4.17: Variation of inner and outer duty-cycles of the trapezoidal SWG segments.

With the maximum duty-cycle ratio set, the $\mathrm{X} / \mathrm{Y}$ coordinates, the angles, and curvature can be used to calculate the positions and side lengths of all 77 SWG segments. This data is imported into Lumerical FDTD and the geometries are generated using a script. 


\subsubsection{Radial Bridging Strips}

The goal of the radial bridging strips is two-fold. The first is to add a finer degree of control to the index gradient on top of the coarse control from tailoring the trapezoidal segments. The second is to compensate for the lack of a partially etched layer, which would normally increase the effective index of the bend. By having both trapezoidal and radial SWG segments, a fully-etched bend can still have a large enough effective index to confine higher order modes that would normally radiate out of the bend.

The radial strips run perpendicular to the radius of the bend and vary in width depending on the curvature at given point along the bend. The strips have the same width at the inputs and outputs of the bend, but the widths are tapered as a function of curvature until a maximum/minimum value at the $45^{\circ}$ midpoint. Much like how the initial trapezoidal SWGs have a duty-cycle of $60 \%$, a similar spacing was chosen for the perpendicular bridging-strips. A waveguide of width $2.075 \mu \mathrm{m}$ can fit eight strips of width $170 \mathrm{~nm}$, resulting in an interstrip spacing of approximately $103 \mathrm{~nm}$ (duty-cycle of 62\%). This is desirable as the spacing can be reduced on the inner edge of the bend to shape the index gradient, while still being above the minimum feature size of $60 \mathrm{~nm}$.

With eight radial bridging-strips it was decided to split them into two groups of four: the inner and outer strips. A maximum inner width and a minimum outer width would be used to define all 8 strips, with the 4 inner strips increasing in width linearly up to the maximum width, and the 4 outer strips decreasing in width down to the minimum width (see Fig. 4.18). This allows 2 degrees of freedom when running parameter sweeps as the inner and outer strips can be tuned separately. 
a)

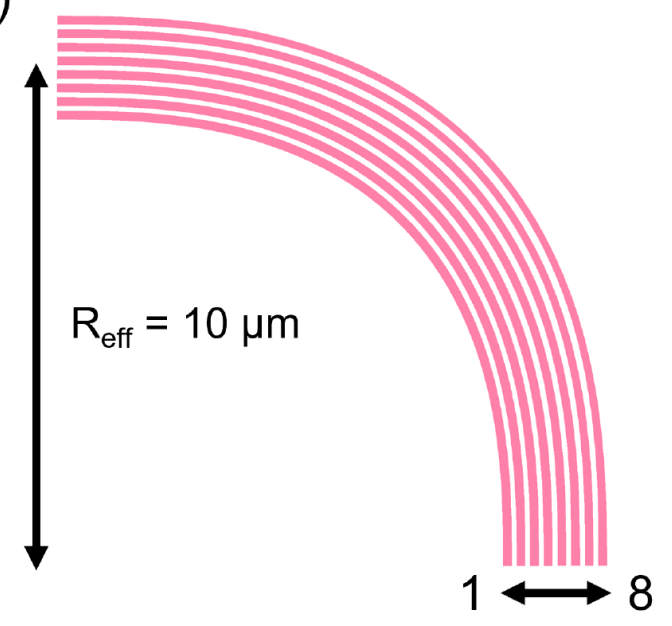

b)

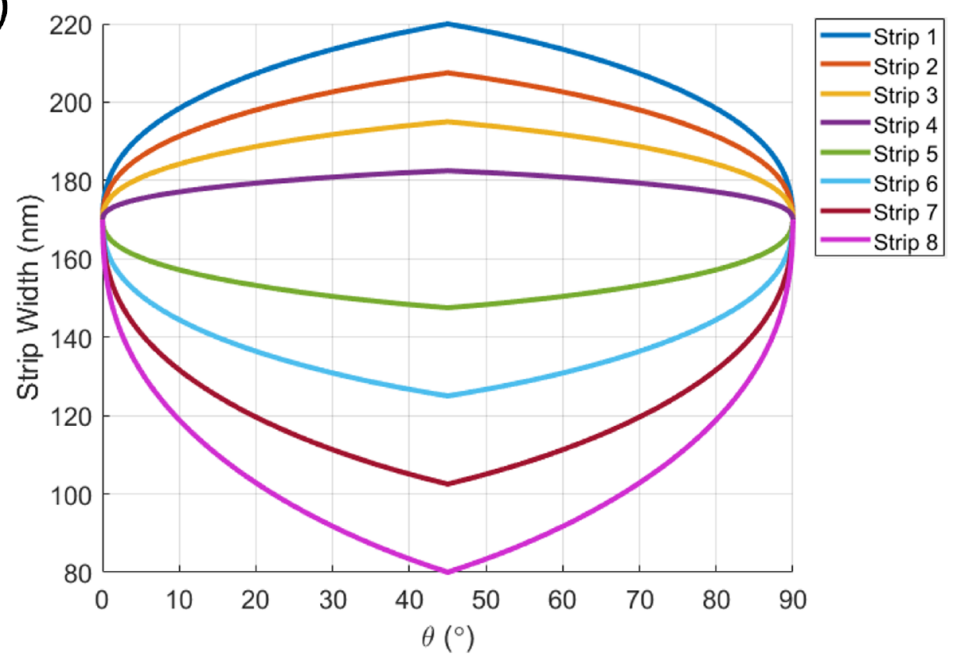

Figure 4.18: a) Scale diagram of eight radial bridging strips with tapered widths. b) Change in strip width as a function of angle.

The bridging strips are calculated using the same process as the optimized curve from section 4.7.1. The only difference is the radius of each strip is larger or smaller depending on its position along the inner or outer edge (the range of the strip radii is 8.96-11.03 $\mu \mathrm{m}$ ).

\subsubsection{Multimode Fishbone Taper}

Previous implementations of SWG-based multimode bends have forgone the inclusion of tapers as they typically include a partially-etched SWG layer. The height of the partiallyetched SWG segments is only a small fraction (ex: 15\%) of the total silicon height, meaning most of the modal field was confined to the slab layer. A shallow partial etch-depth is preferable to a deep partial-etch as it reduces scattering of TM modes, and the reduced vertical asymmetry mitigates TM mode-hybridness. The main advantage however is that the bend can transition from solid waveguide to SWG-bend abruptly, with minimal reflection losses due to the relatively small index mismatch. A solid waveguide that abruptly transitions 
into a fully-etched SWG bend will exhibit non-negligible reflection losses, potentially limiting the operational bandwidth. This can potentially be mitigated by designing a taper that transitions between the solid waveguide and SWG-bend, but at the expense of an increased footprint.

A photonic taper is a gradual change in waveguide geometry that slowly varies the value of $n_{\text {eff }}$ along the direction of propagation. The simplest form of SOI taper is a linear change in the waveguide width. In general longer tapers result in lower losses as the change in width is so small that the change in $n_{\text {eff }}$ is gradual enough that it suppresses reflections. Tapers that convert between solid waveguides to SWGs have existed since the invention of SWGs [11], and generally follow the fishbone structure, as seen in Fig. 4.19. A fishbone taper consists of an SWG interlaced with a central fishbone segment that gradually reduces in size until it reaches the minimum allowed feature size. After this point the taper abruptly transitions to a standard SWG with a slightly larger width to offset for the change in $n_{e f f}$.

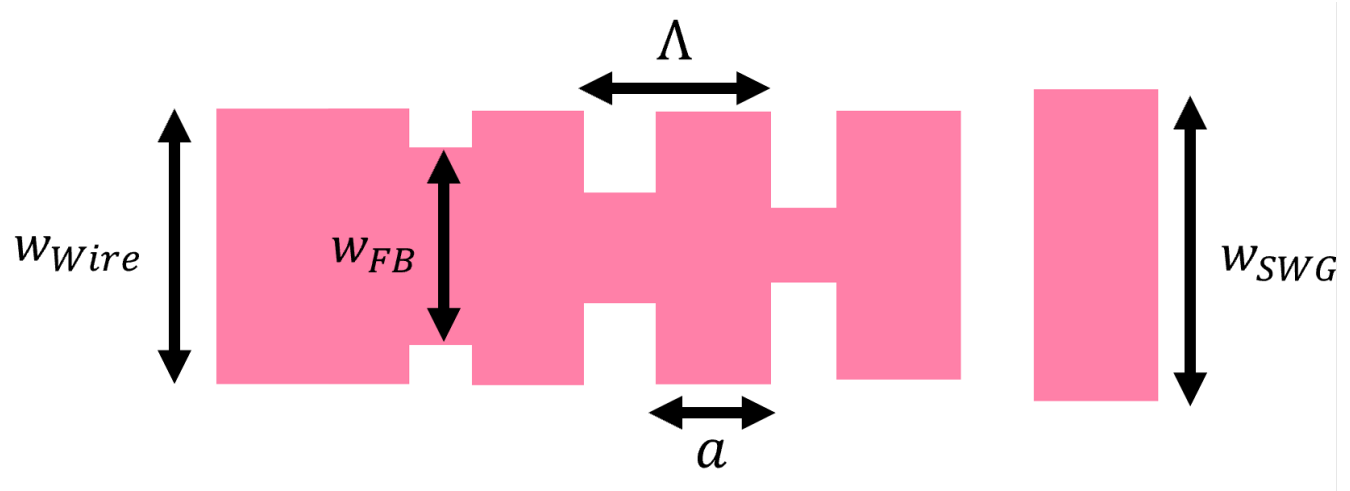

Figure 4.19: A standard single-mode fishbone taper between a solid waveguide and SWG.

Fishbone tapers are typically upwards of $10 \mu \mathrm{m}$ in length in order to minimize loss. Fishbone tapers have been widely demonstrated for the fundamental TE and TM modes, but to the best of my knowledge there has not been a demonstration of a successful multimode 
fishbone taper in a wire-to-SWG system. Multimode tapers for wire-to-wire or SWG-to-SWG systems are as simple as linearly increasing the width until additional modes are supported. Furthermore, fishbone tapers have been used when converting single-mode wire waveguides into SWG-based multimode interference (MMI) devices [51], or when converting between a wire waveguide to a SWG-slot waveguide [52]. However the conventional fishbone taper cannot be optimized for more than one mode at a time because of the way modal fields spread through multimode waveguides. Every mode order has a unique $n_{\text {eff }}$ value that determines how the modal fields spread through a waveguide, leading to the mode orders becoming asynchronous. A successful multimode fishbone taper should maintain the synchronicity of the field profiles between all mode orders. A comparison between these two types of tapers is shown in Fig. 4.20.
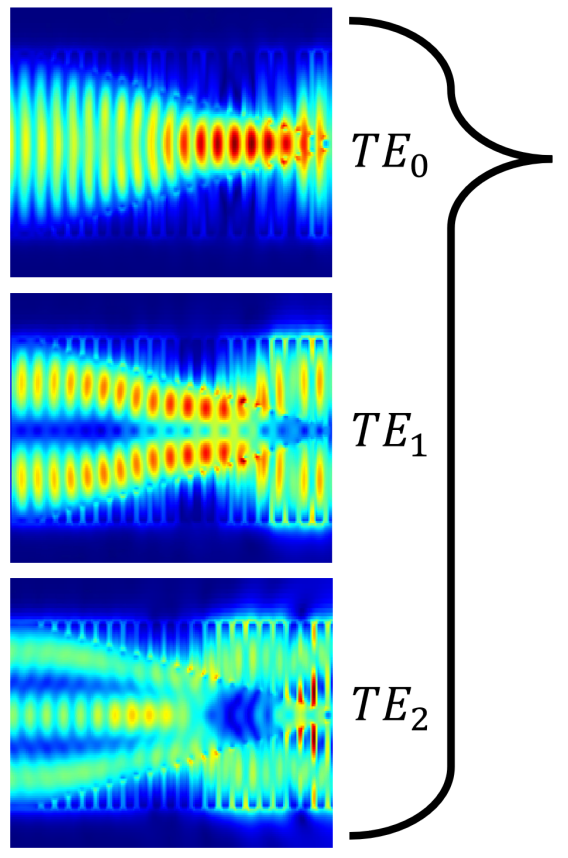

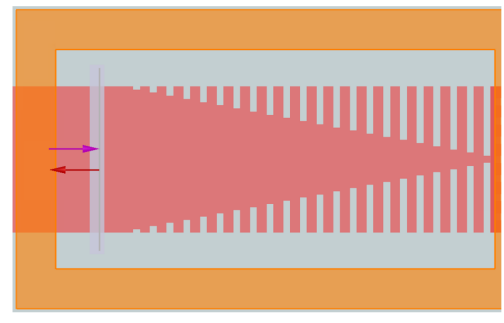

Standard fishbone taper

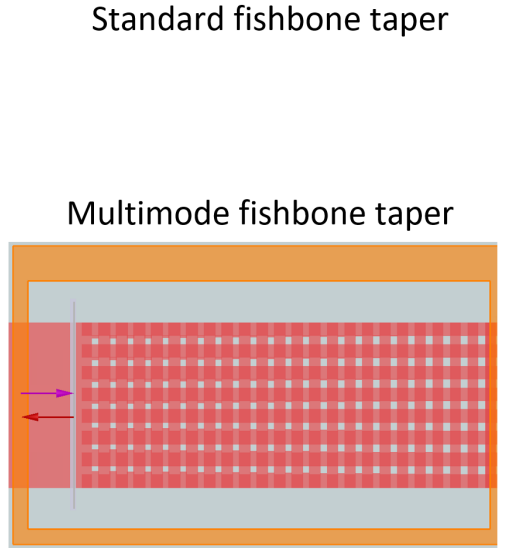

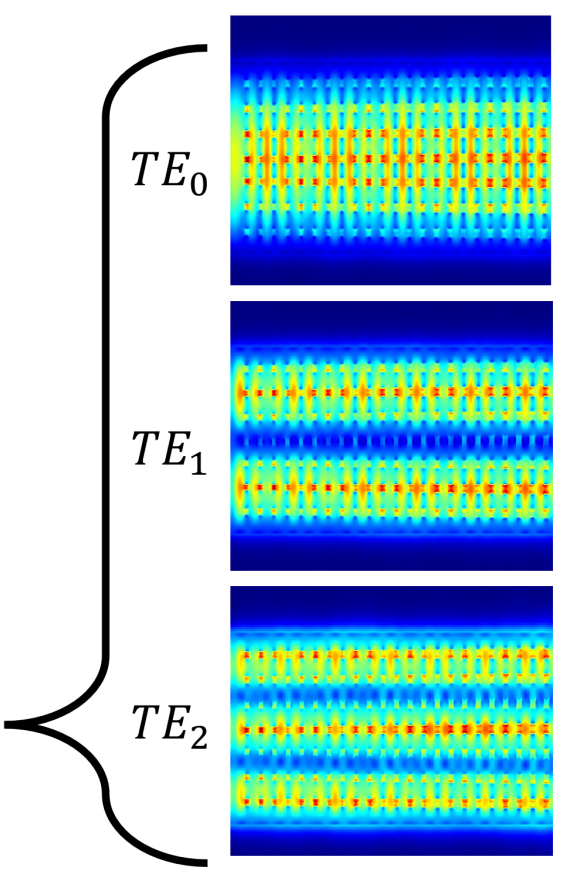

Figure 4.20: A demonstration of a conventional fishbone taper and a multimode equivalent fishbone taper. 
We propose a multimode taper that uses one fishbone for every radial bridging strip, as depicted in Fig. 4.21. The taper begins with holes the size of the minimum feature size, which are then gradually increased until they match the beginning of the bend. The key advantage of this taper design is that it does not need to be modified in order to scale up to accommodate additional higher order modes.

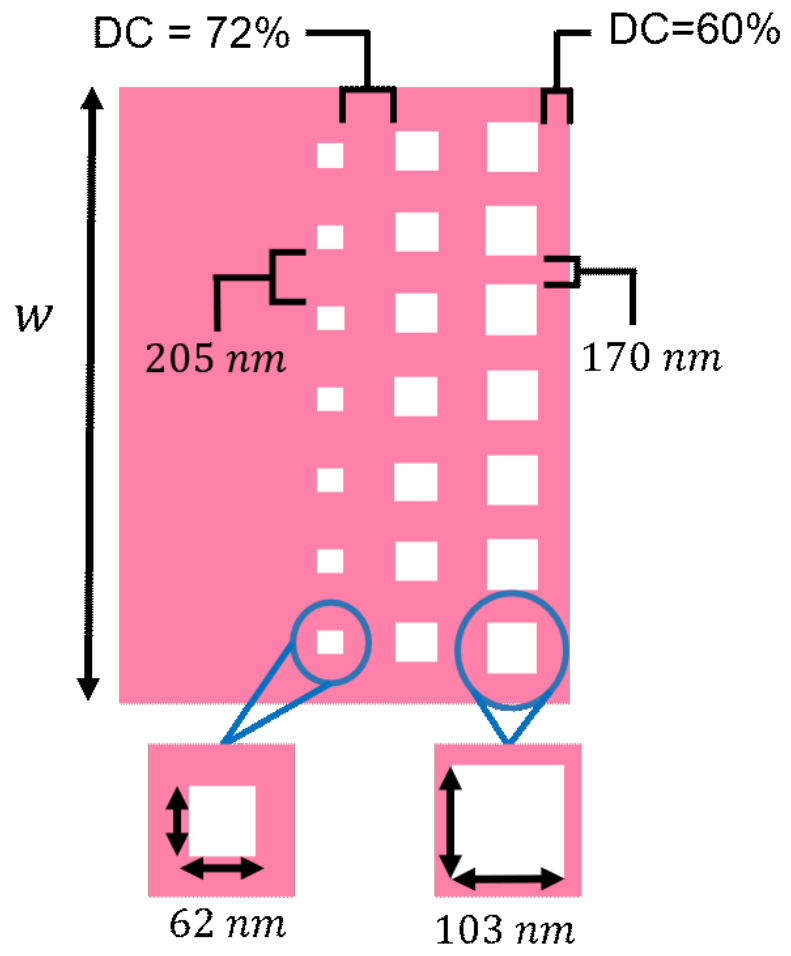

Figure 4.21: The designed multimode fishbone taper. The solid waveguide transitions into the SWG bend by tapering the size of the etched holes up from the minimum feature size to the size at the input of the bend.

A solid wire waveguide abruptly transitioning into a waveguide with holes $103 \mathrm{~nm}$ x 103 $\mathrm{nm}$ in size will naturally produce reflections, leading to a Fabry-Perot cavity effect within the bend. This resonator effect can be lessened by tapering the size of the holes from the 
absolute minimum possible size $(62 \mathrm{~nm} \times 62 \mathrm{~nm})$ up to the holes at the input of the bend (103 nm x $103 \mathrm{~nm})$. A taper length of $5 \mu \mathrm{m}$ was chosen as a reference benchmark, increasing the total footprint from $10 \mu m \times 10 \mu m$ up to $15 \mu m \times 15 \mu m$. The performance of bends without tapers was compared to bends with tapers in section 4.8.3.

\subsection{D FDTD Simulations of Bend}

\subsubsection{Band Structure Calculations}

Multimode bends that use a partial etch have the advantage of a low effective index mismatch between the bus waveguide and the SWG bend waveguide. The majority of the modal field is contained in the slab waveguide, with only minor reflections due to mismatch with the partially-etched SWGs. A fully-etched SWG waveguide bend presents a challenge as the mismatch between the bus waveguide and SWG bend section is much higher, resulting in stronger reflections. In this work the choice of the optimal bend ensures that the contribution of the modal mismatch due to curvature is minimized. However there will still be reflections at the interface due to the transition between the effective index of the bus waveguide and bridged-SWG. The bus waveguide will have a larger $n_{\text {eff }}$ for every mode order compared to the equivalent $n_{\text {eff }}$ for the bridged-SWG section. Quantifying this mismatch in $n_{\text {eff }}$ is critical for evaluating unwanted Fabry-Perot cavity effect, as well as the utility of the SWG taper section.

The effective index of the bus waveguides were calculated using a Lumerical MODE in the bandwidth of interest (1500-1600 nm) at the design width of $2.075 \mu \mathrm{m}$ for both waveguide heights. Obtaining the $n_{\text {eff }}$ values for the bridged-SWG section required a FDTD unitcell simulation with Bloch-boundary conditions, as previously discussed in section 3.4. The 
wavelength source was swept by varying the normalized Bloch wavenumber $K$ from 0 to 0.5 (i.e. the first Brillouin zone) for each mode order. From this sweep the phase velocity was extracted, and subsequently the value of $n_{\text {eff }}$. This step was performed for both the bridgedSWG at the input of the bend, as well as the bridged-SWG taper. The bandstructure was also calculated for the SWGs with the bridges removed, but the majority of the mode orders were not supported. The resulting $n_{\text {eff }}$ wavelength relationships shown in Figs. 4.22 and 4.23 .
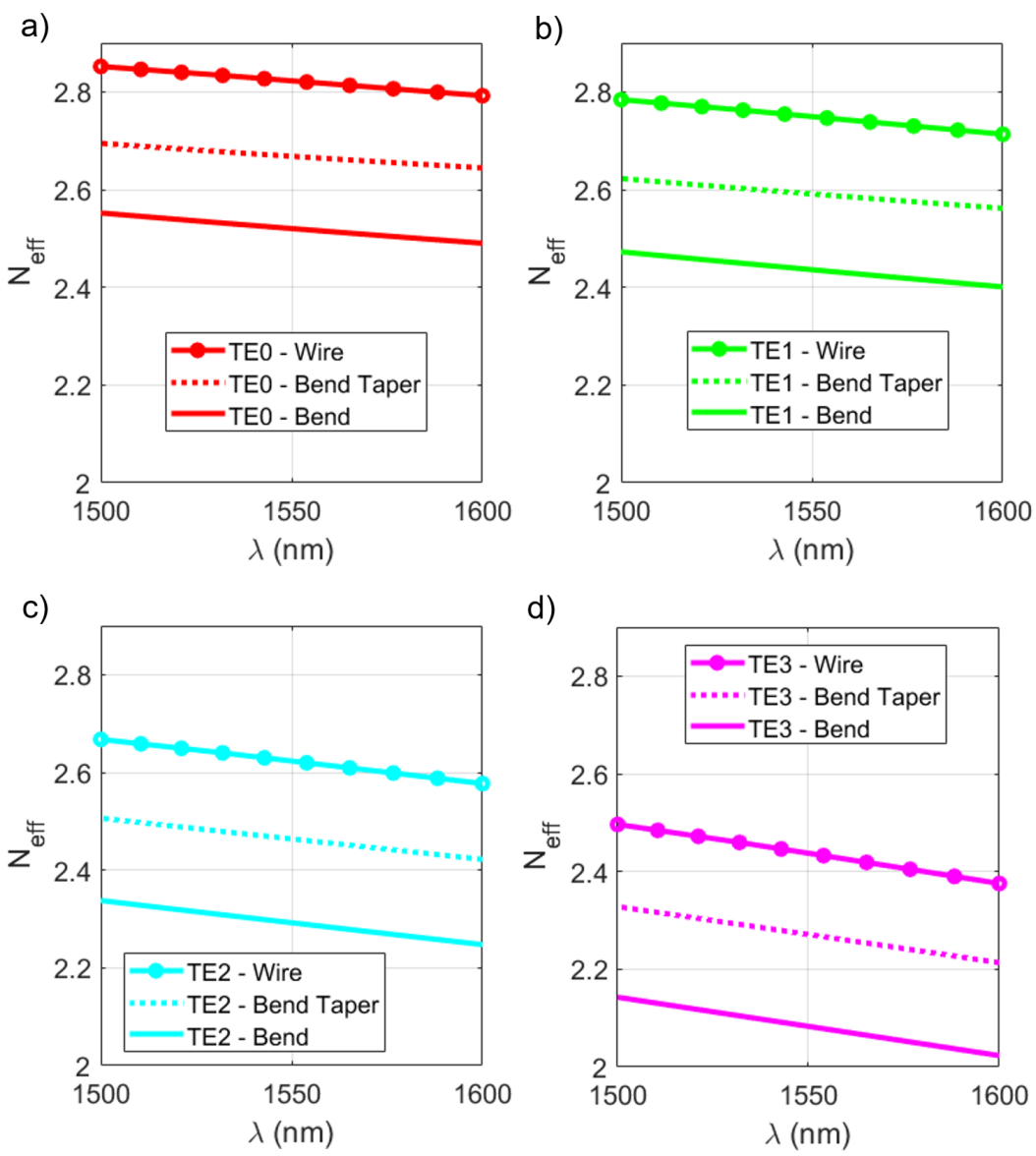

Figure 4.22: Wavelength response of $n_{\text {eff }}$ vs wavelength for the $220 \mathrm{~nm}$ variant: a) $\mathrm{TE}_{0}$, b) $\mathrm{TE}_{1}$, c) $\left.\mathrm{TE}_{2}, \mathrm{~d}\right) \mathrm{TE}_{3}$. 
a)

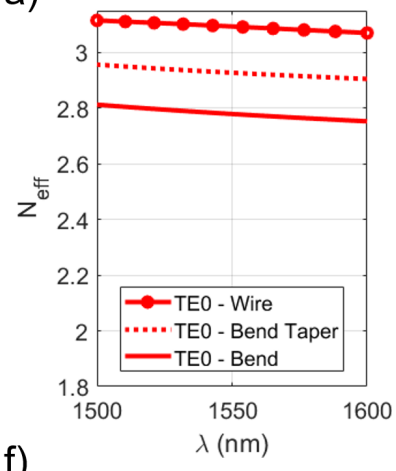

f)

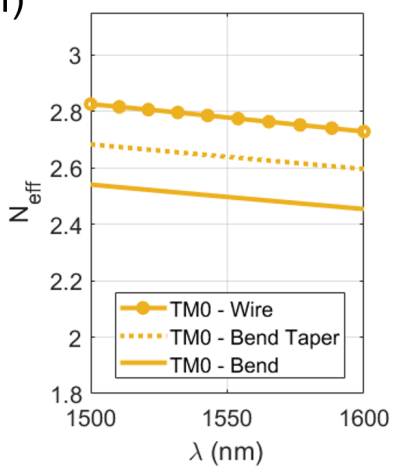

b)

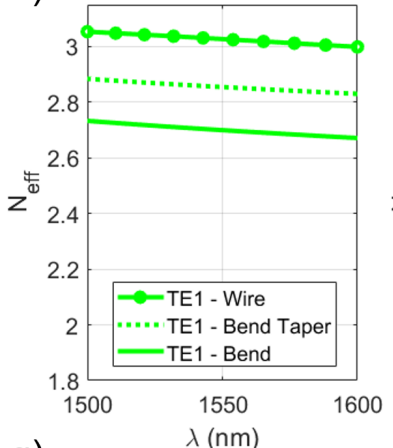

g)

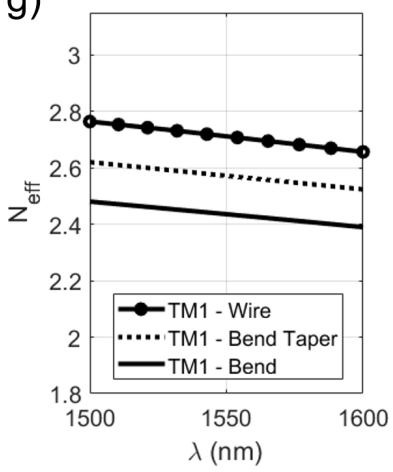

c)

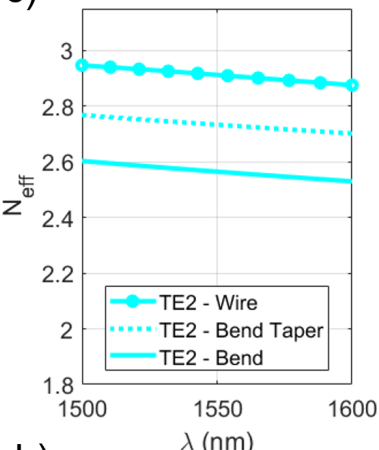

h)

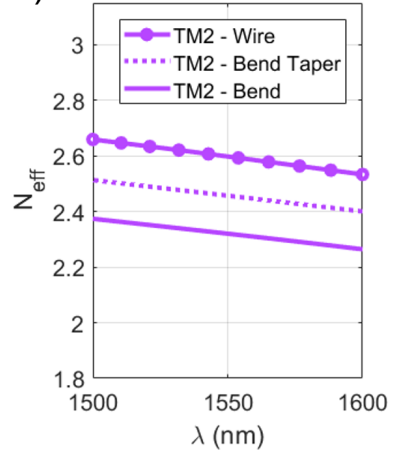

d)

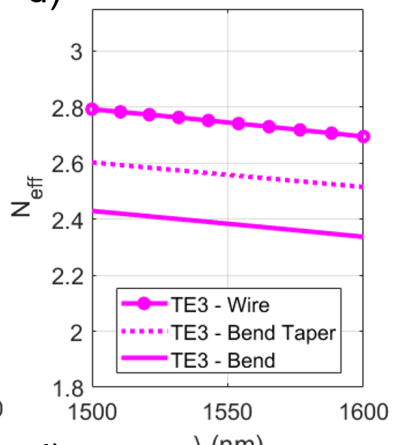

i)

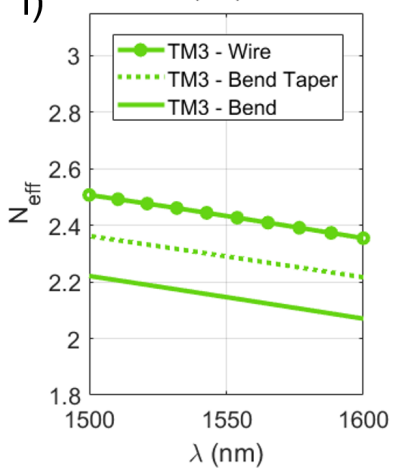

e)

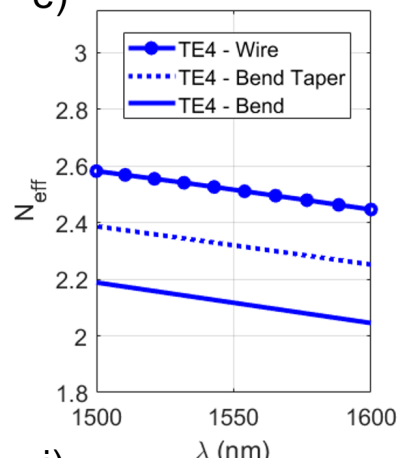

j)

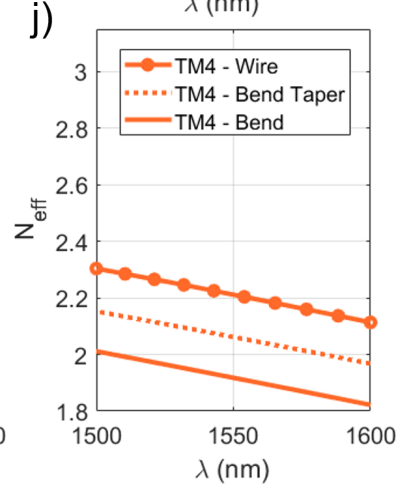

Figure 4.23: Wavelength response of $n_{\text {eff }}$ vs wavelength for the $340 \mathrm{~nm}$ variant: a) $\mathrm{TE}_{0}$, b) $\mathrm{TE}_{1}$, c) $\mathrm{TE}_{2}$, d) $\mathrm{TE}_{3}$, e) $\mathrm{TE}_{4}$, f) $\mathrm{TM}_{0}$, g) $\mathrm{TM}_{1}$, h) $\mathrm{TM}_{2}$, i) $\mathrm{TM}_{3}$, j) $\mathrm{TM}_{4}$.

It can be seen that all modes exhibit a decreasing $n_{e f f}$ with respect to wavelength, regardless of structure. As expected the bus waveguides have the highest $n_{\text {eff }}$ for every mode order, followed by the SWG bridged-taper, and finally the SWG-bridged bend. The SWG bridged-taper is shown to decrease the index-mismatch by up to half. Using Fresnel's reflection equation at normal incidence (Eq. (4.13)), this reduces the percentage of reflected power from approximately $0.5 \%$ to $0.1 \%$. Given that the bend acts like a mild resonator with many repeated reflections, this reduction in reflection percentage is a significant improvement. 


$$
R=\left|\frac{n_{e f f, 1}-n_{e f f, 2}}{n_{e f f, 1}+n_{e f f, 2}}\right|^{2}
$$

\subsubsection{Optimization Process}

Prior to this point several key variables have been locked-in to the designs. Some of these variables were chosen for performance considerations including the effective radius (10 $\mu m)$, the waveguide width $(2.075 \mu \mathrm{m})$, and the waveguide height (220 and $340 \mathrm{~nm})$. Other variables were chosen as a trade-off between performance and fabrication considerations such as the SWG pitch and initial duty-cycle (220 nm and 60\%), as well as the number and initial width of the bridged-SWG strips (8 and $170 \mathrm{~nm}$ ). The final multimode bend design is therefore determined by 4 remaining parameters: The inner and outer duty-cycles of the trapezoidal SWGs, and the widths of the inner and outer bridging-strips.

Specifically, these dimensions are determined by setting their values at the point of maximum curvature, i.e. $\theta=45^{\circ}$. The inner and outer duty-cycles both begin at a value of $60 \%$, but are slowly tapered to their minimum values as a function of the curvature. The bridging-strips begin with a width of $170 \mathrm{~nm}$, but taper down or up in size depending on whether they are one of the outer or inner strips. The ratio of the inner and outer duty-cycles of the trapezoidal segments is a critical parameter that sets the default sharpness of the bend's index profile. The process followed in this work was to set an initial value for $\eta_{1} / \eta_{2}$ and further optimize by performing a parameter sweep of the inner and outer bridging-strips.

For a pitch of $220 \mathrm{~nm}$ the maximum duty-cycle allowed by the fab-specifications is $73 \%$, meaning an initial value of $60 \%$ leaves a reasonable buffer. Because the variable curvature leads to a denser spacing at $\theta=45^{\circ}$ the inner duty-cycle must be reduced from $60 \%$ down 
to $50 \%$ to maintain the desired buffer. The minimum outer duty-cycle can be set as low as $28 \%$ while still complying with the feature size constraint, meaning a possible range of $28-50 \%$. Following some initial simulation attempts the chosen ratio for $340 \mathrm{~nm}$ was $0.5 / 0.35$ $=1.43$, and the chosen ratio for $220 \mathrm{~nm}$ was $0.5 / 0.42=1.19$. The following section details the optimization procedure of the bridge-strips.

\section{Simulation Workflow}

The optimization process required three main steps, and is illustrated in Fig. 4.24. Firstly Matlab is used to generate dozens of sets of coordinates for the bridged strips, one data set for each width that will be part of the parameter sweep. Secondly these coordinates are imported into KLayout using a Ruby script and then converted into polygons in a GDS file. The four inner and four outer strips are saved as separate polygon groups as are they swept independently of each other. Each set of strips is saved as a different layer in the GDS file with a unique layer number, resulting in a final GDS file with approximately 100 total bridging-strip variants. Finally a script written for Lumerical FDTD loads from the GDS file one set of inner strips and one set of outer strips, after which it runs a simulation $(\lambda$ $=1550 \mathrm{~nm}$ ), saves the data, and iterates to the next combination of strips. This process is performed for every combination of inner and outer strips and for each mode order of interest. 


\section{Radius, width, duty-cycle ratio \\ Matlab \\ $\mathrm{X} / \mathrm{Y}$ coordinates, Angle \& Curvature}

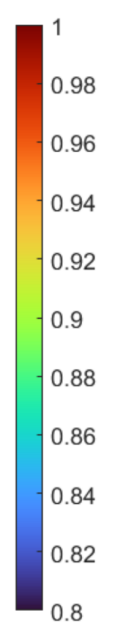

\section{Mode Overlap}

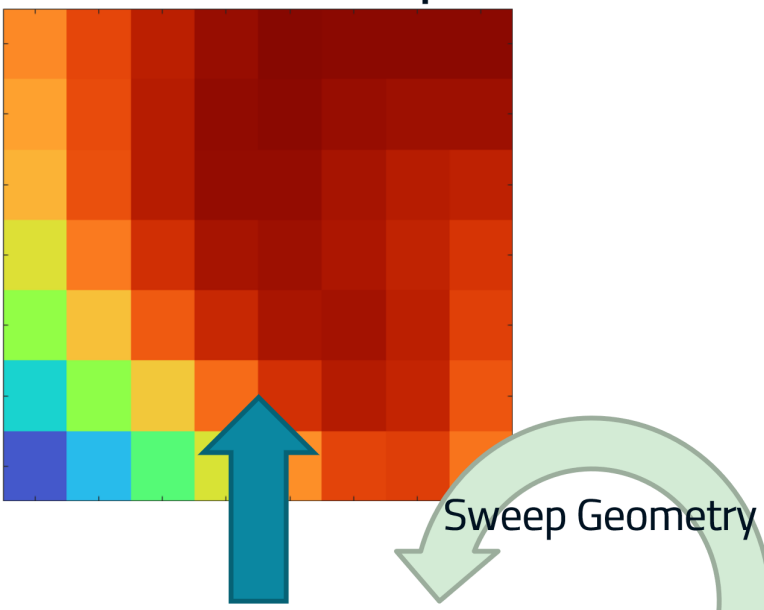

\section{Klayout (Ruby Scripting)}

\section{GDS file}

Figure 4.24: Flowchart demonstrating the workflow of the multimode bend optimization process.

\section{Mode Overlap}

In order to efficiently perform parameter sweeps for optimization a figure of merit had to be selected. The goal of a multimode bend is ensure that for every mode order the field profile at the input matches that at the output. This can be assessed visually by observing a plot of the field distribution, either from a top-down perspective or by comparing the cross-sections of the input and output. A visual assessment is helpful for initial simulations, but far too inefficient when performing dozens of parameter sweeps. A single numerical figure of merit (FOM) would allow for easy data visualization and speed up the optimization process. One potential FOM was the $S_{21}$ parameter, which is a value from 0-1 assessing the power transfer 
between the input and output measurement ports. The downside is that the $S_{21}$ parameter can vary quite a bit with respect to wavelength due to the mild resonator effect of the bend, and a parameter that is less wavelength sensitive is preferable. It was decided that mode overlap (defined earlier in Eq. (4.6)) was the most suitable FOM.

Mode overlap is a positive value within the range of $0-1$, representing the percentage of transferred power between two modal fields. Lumerical FDTD has a built-in mode overlap calculation that can be applied to two monitors which are oriented on the same axis. In the context of a bend this feature can be used to assess how well the bend maintains the field profile of any given mode. Two monitors are placed at the output of the bend simulation, one immediately after the bend and one further along the output waveguide (approximately $3 \mu \mathrm{m}$ ). If the bend successfully maintains the balanced mode profile the peaks of the field profile will arrive in-synch. If they are in-synch then they will continue to propagate with minimal power transfer between peaks, leading to a mode overlap value close to 1 . If the modal peaks arrived out of synch, power will gradually begin to transfer between peaks, and the resulting mode will have a different $n_{\text {eff }}$ than the input, reducing the effectiveness of an MDM system. Through qualitative inspection a target mode overlap of $95 \%$ was established. Greater than $95 \%$ was ideal, with an absolute minimum acceptable value of $90 \%$ for a mode to be considered satisfactory. See Fig. 4.25 for a visual example of this threshold. 

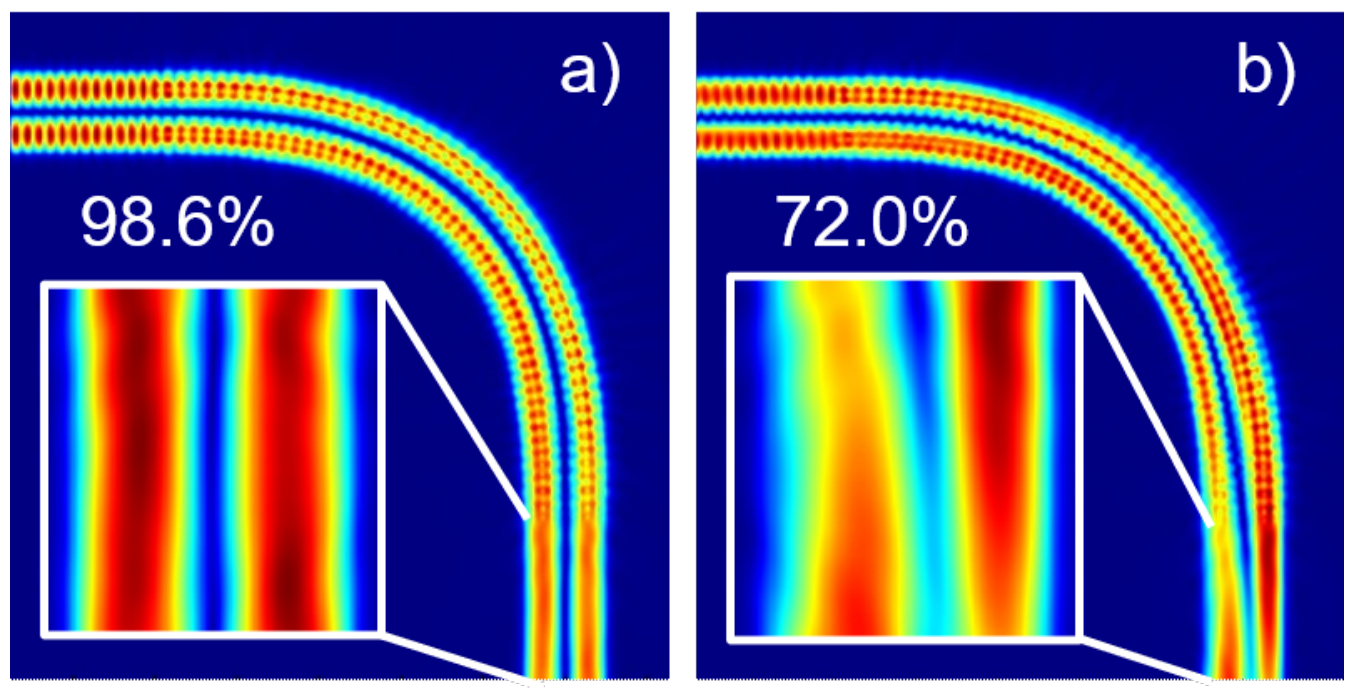

Figure 4.25: The $\mathrm{TM}_{1}$ input injected into a $340 \mathrm{~nm}$ bend with a) excellent overlap, b) poor overlap.

\section{Parameter Sweep Results}

To optimize the bend geometry every mode order had to achieve a mode overlap of $90 \%$ or higher. The process was as follows: A mode order was selected to be optimized, and a script individually loaded the radial SWG strips from the prepared GDS file and ran each simulation, resulting in a series of heat maps. For the $340 \mathrm{~nm}$ variant the results for the TE modes are shown in Fig. 4.26 and for TM in Fig. 4.27. 

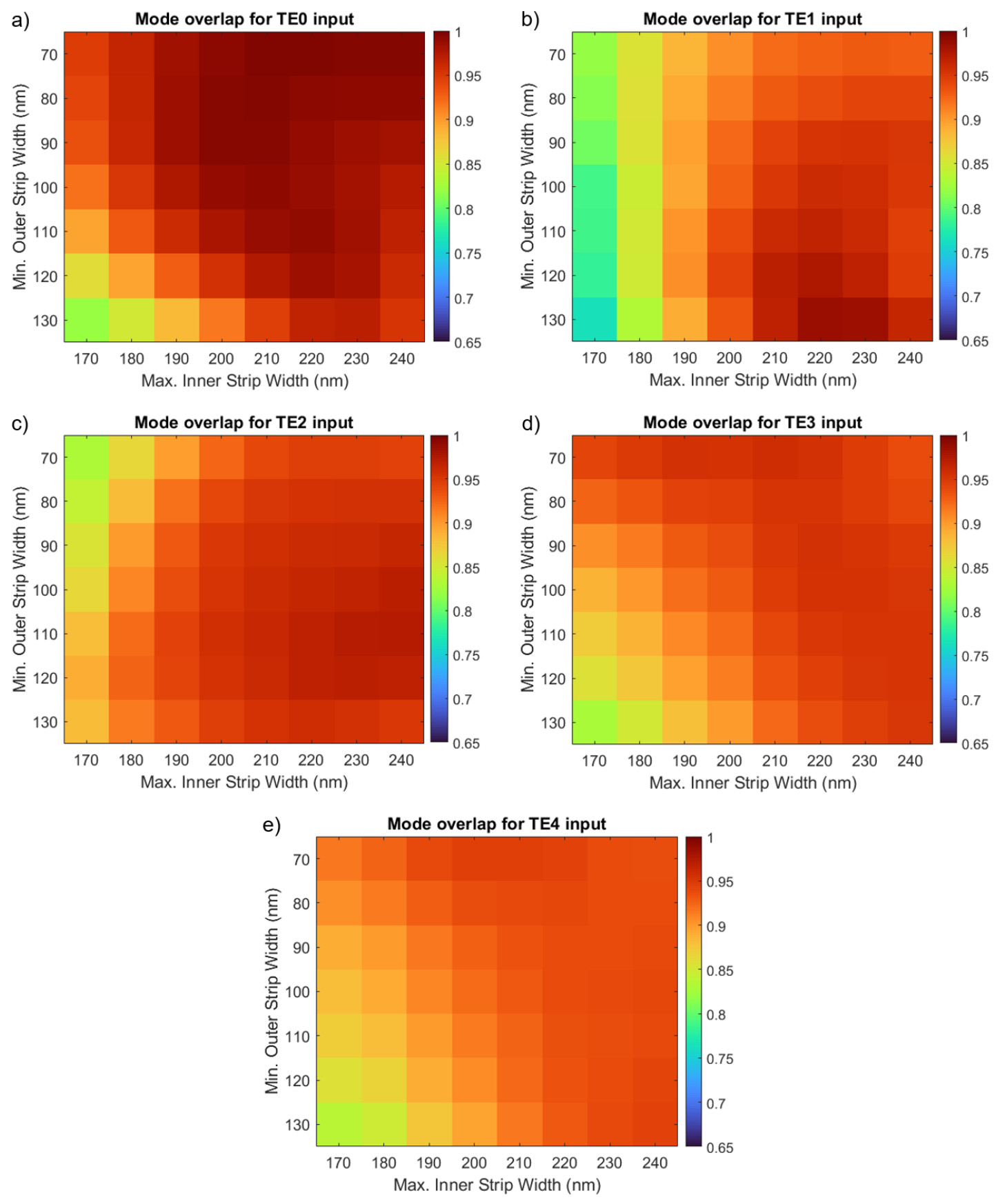

Figure 4.26: Parameter sweep evaluating mode overlap at the output of the $340 \mathrm{~nm}$ bend variant: a) $\mathrm{TE}_{0}$, b) $\mathrm{TE}_{1}$, c) $\left.\mathrm{TE}_{2}, \mathrm{~d}\right) \mathrm{TE}_{3}$, e) $\mathrm{TE}_{4}$. 

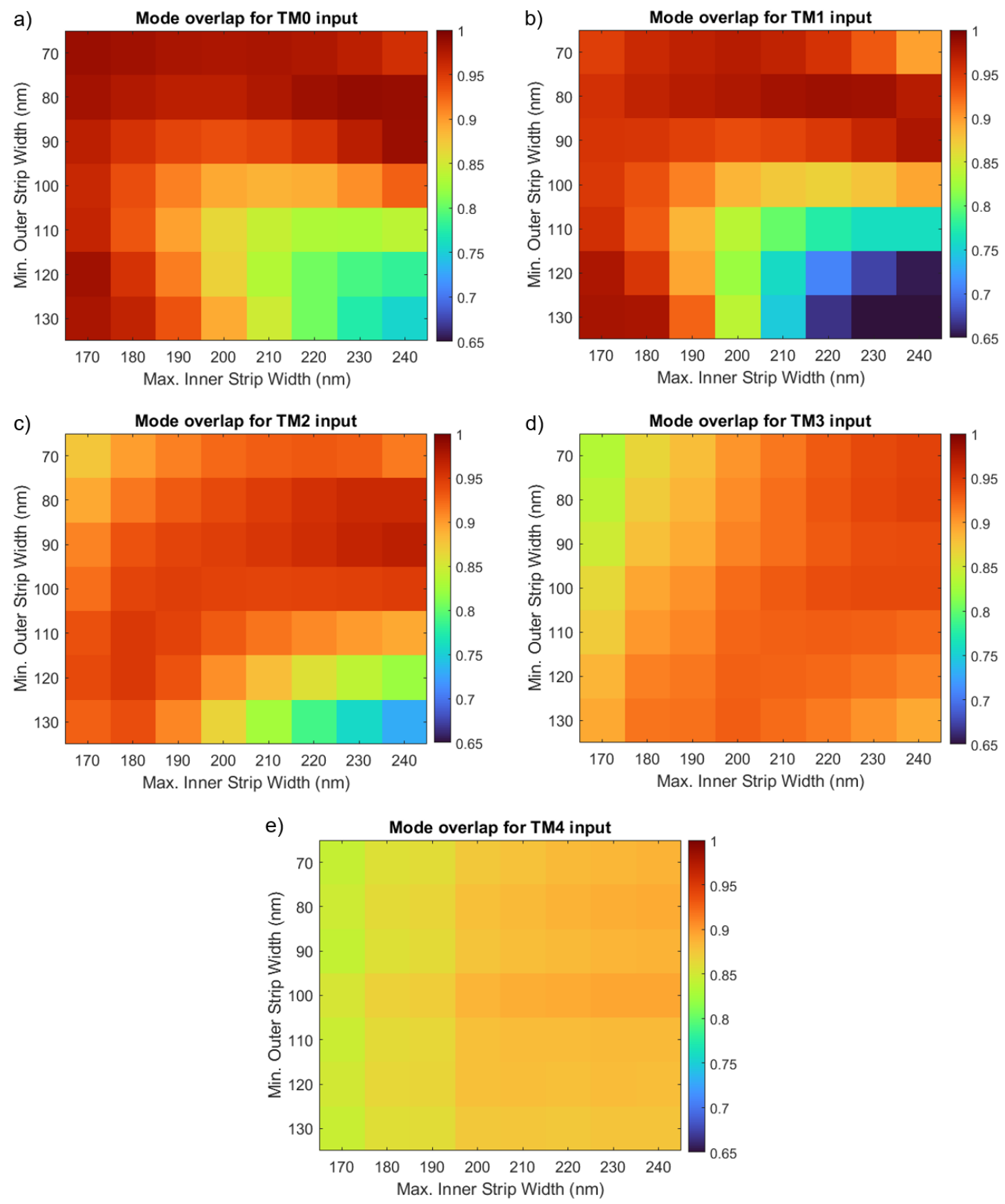

Figure 4.27: Parameter sweep evaluating mode overlap at the output of the $340 \mathrm{~nm}$ bend variant: a) $\mathrm{TM}_{0}$, b) $\mathrm{TM}_{1}$, c) $\mathrm{TM}_{2}$, d) $\mathrm{TM}_{3}$, e) $\mathrm{TM}_{4}$. 
Each mode order has a different optimal geometry, but the upper right quadrant of the mode overlap matrices is the best compromise for all modes. It should be noted that for the $\mathrm{TM}_{4}$ mode the overall performance is lower because it has the lowest $n_{\text {eff }}$ value and a non-negligible amount of power is lost radiating out of the bend. For this reason the $\mathrm{TM}_{4}$ was not considered when selecting the optimal geometry. The geometry combination that provided the best compromise between modes was a minimum width of $80 \mathrm{~nm}$ and a maximum width of $220 \mathrm{~nm}$. For this geometry the smallest mode overlap ratios were the $\mathrm{TM}_{3}$ mode with $93.2 \%$ and the $\mathrm{TE}_{1}$ mode with $93.7 \%$. All other modes had mode overlap values above $95 \%$.

A similar parameter sweep was performed to optimize the $220 \mathrm{~nm}$ bend variant for the first four TE modes, and the resulting heat maps are shown in Fig. 4.28. One immediately visible difference is that the 220 bend variant is much less sensitive to parameter sweeps, with overall higher mode overlap values. This aligns with the pattern established in section 4.6, with the $340 \mathrm{~nm}$ bend variant being more susceptible to mode profile distortion. The geometry that compromised the best for all four modes was a minimum width of $130 \mathrm{~nm}$ and a maximum width of $180 \mathrm{~nm}$. For this geometry the smallest mode overlap ratio was the $\mathrm{TE}_{3}$ mode with $91.2 \%$, with all other modes having overlap values above $95 \%$. A summary of the of the optimized geometry parameters is included in table 4.1. 


\section{SWG Engineered Multimode Waveguide Bends}
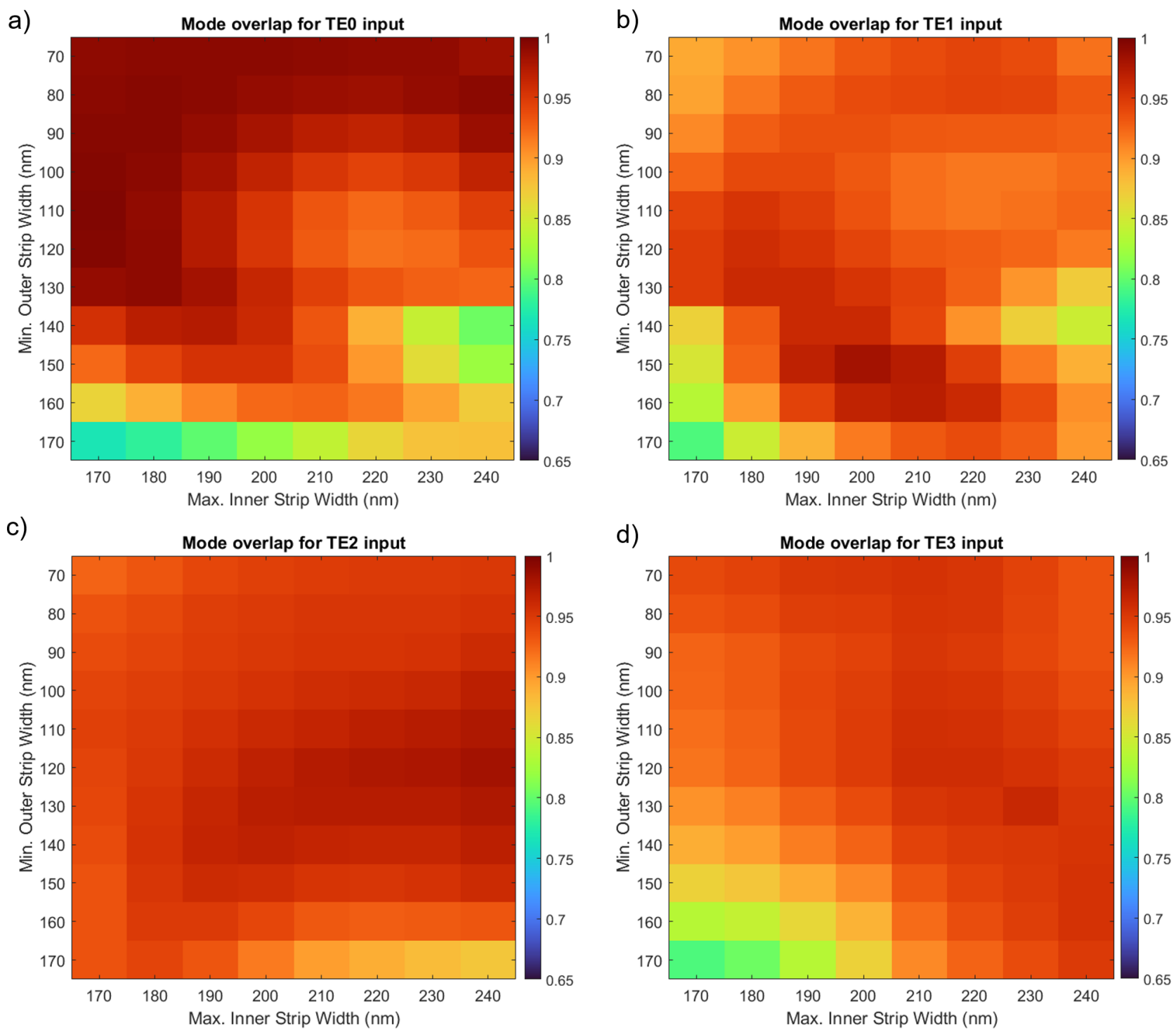

Figure 4.28: Parameter sweep evaluating mode overlap at the output of the $220 \mathrm{~nm}$ bend variant: a) $\mathrm{TE}_{0}$, b) $\mathrm{TE}_{1}$, c) $\mathrm{TE}_{2}$, d) $\mathrm{TE}_{3}$. 
Table 4.1: Optimized geometries for the $340 \mathrm{~nm}$ and $220 \mathrm{~nm}$ multimode bends. The initial duty-cycle is $60 \%$ for both cases and the starting strip width is $170 \mathrm{~nm}$ for both cases.

\begin{tabular}{c|c|c|c|c}
\hline Thickness (nm) & Max. inner DC & Min. outer DC & Max. inner width (nm) & Min. outer width (nm) \\
\hline 340 & $50 \%$ & $45 \%$ & 220 & 80 \\
220 & $50 \%$ & $42 \%$ & 180 & 130 \\
\hline
\end{tabular}

\subsubsection{Optimized Geometries}

\section{Field Distributions}

The FDTD field profile simulations of the bends are picture in Fig. 4.29 and Fig. 4.30 for the $340 \mathrm{~nm}$ and $220 \mathrm{~nm}$ bends, respectively.
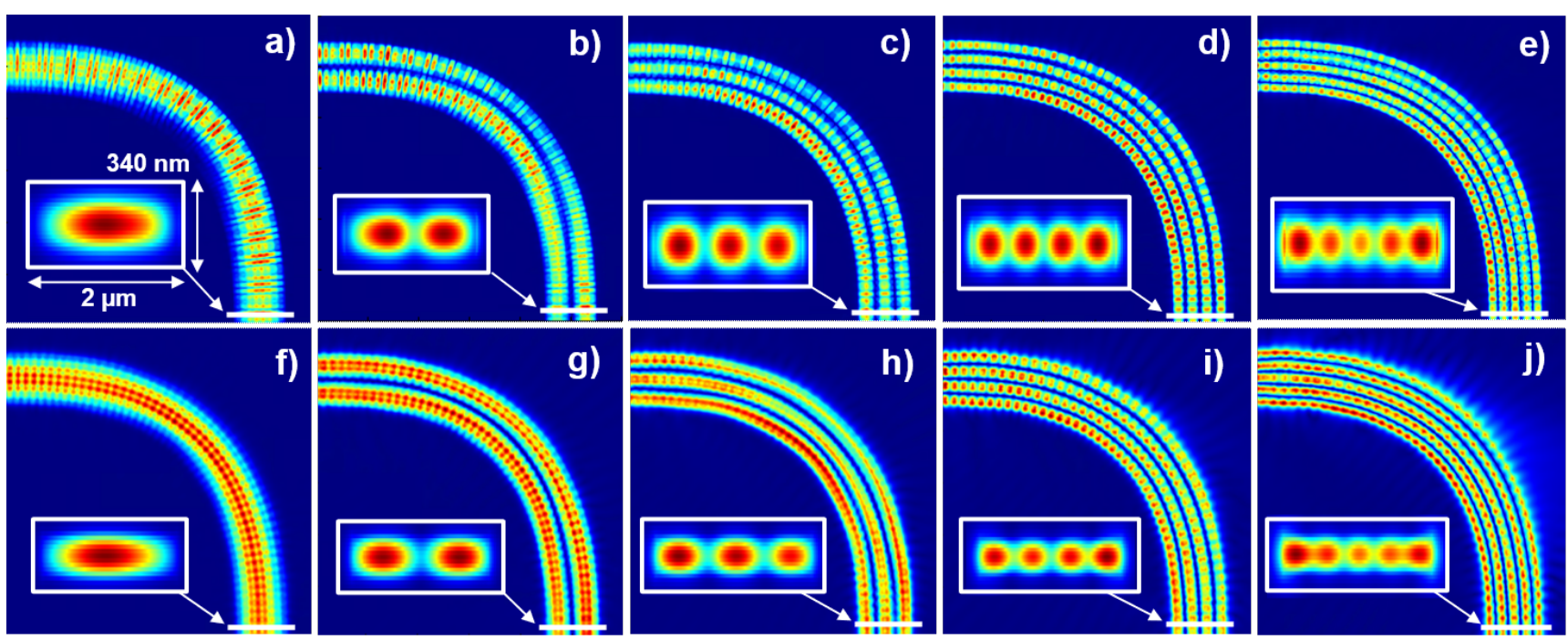

Figure 4.29: Mode overlap optimization results for $340 \mathrm{~nm}$ bend variant: a) $\mathrm{TE}_{0}$, b) $\mathrm{TE}_{1}$, c) $\mathrm{TE}_{2}$, d) $\mathrm{TE}_{3}$, e) $\mathrm{TE}_{4}$, f) $\mathrm{TM}_{0}$, g) $\mathrm{TM}_{1}$, h) $\mathrm{TM}_{2}$, i) $\mathrm{TM}_{3}$, j) $\mathrm{TM}_{4}$. 

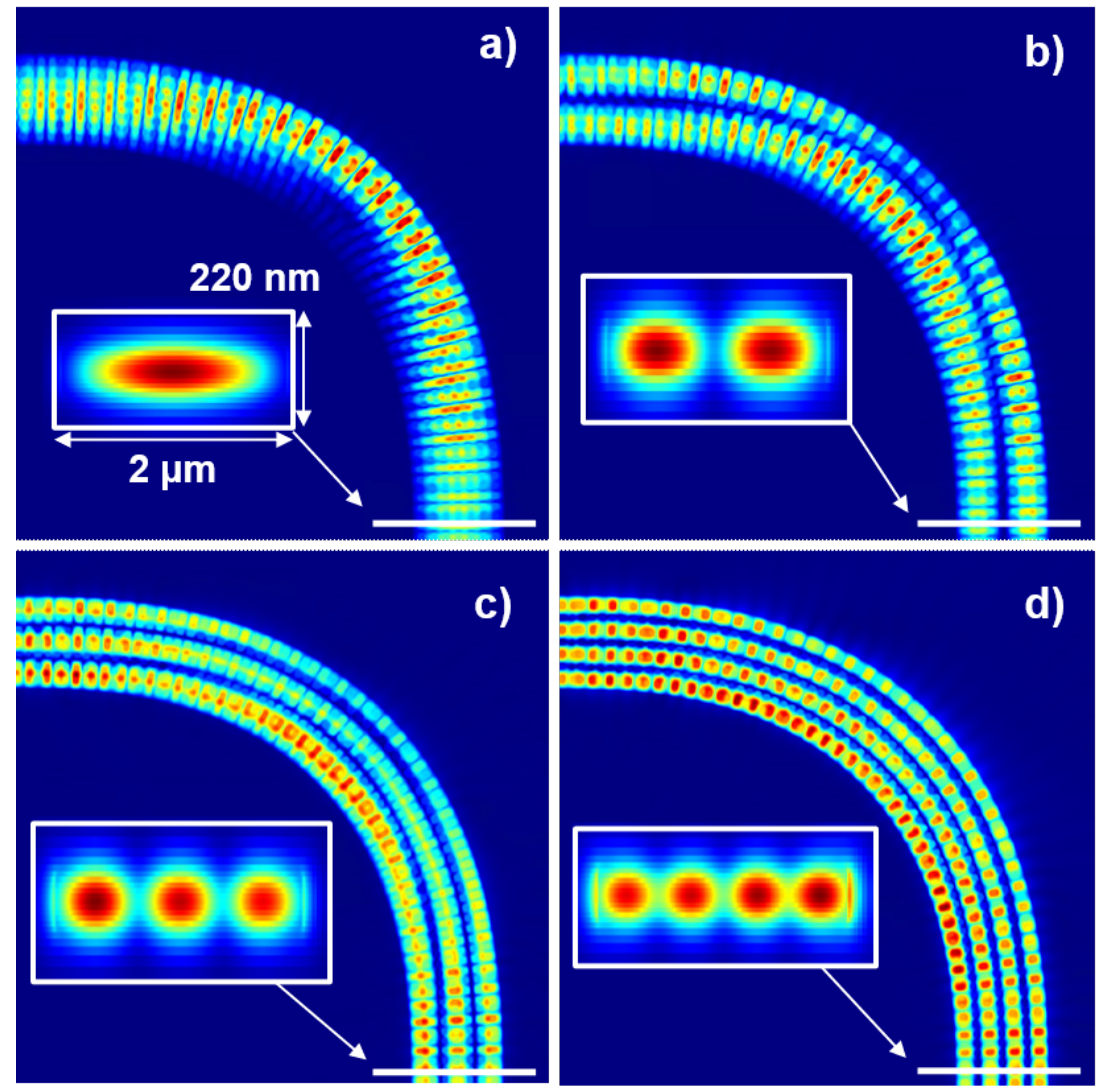

Figure 4.30: Mode overlap optimization results for $220 \mathrm{~nm}$ bend variant: a) $\mathrm{TE}_{0}$, b) $\mathrm{TE}_{1}$, c) $\left.\mathrm{TE}_{2}, \mathrm{~d}\right) \mathrm{TE}_{3}$.

\section{Transmission Spectra}

The bends were designed at an operational wavelength of $1550 \mathrm{~nm}$, the standard central wavelength of the telecommunications C-band. A robust bend design should maintain its performance across a large bandwidth to maximize its effectiveness, therefore the transmission spectra across the 1500-1600 $\mathrm{nm}$ range was simulated. The wavelength response of the bends can be quantified by two properties: i) Excess Loss \& ii) Intermodal Crosstalk.

Excess loss is a quantity that combines all potential sources of loss (mode mismatch, 
radiation loss, sidewall scattering) into one single parameter, as defined by Eq. (4.14), where $i$ is the mode order. It is a real and positive value that should ideally be as close to 0 $\mathrm{dB}$ as possible. It can be extracted from a Lumerical FDTD simulation by evaluating the S-parameter between the input mode-injection port and the output port.

$$
E L_{i}=-10 \cdot \log _{10}\left(T_{i i}\right)
$$

Intermodal cross-talk is a quantity that evaluates the total modal power transfer between the injected mode and any other modes supported by the waveguide, as defined by Eq. (4.15), where $i$ and $j$ are two different mode orders. It is a real and negative value that should ideally be as negative as possible. It can similarly be extracted from a Lumerical FDTD simulation by evaluating the S-parameter between the injected input mode and the output port for each mode order of interest. Intermodal crosstalk is often maximized between mode orders with the closest $n_{\text {eff }}$ values, i.e. $i, i+1$, and $i-1$.

$$
C T_{i j}=10 \cdot \log _{10}\left(T_{i j}\right) \text { where } i \neq j
$$

An optimal multimode bend should both minimize excess loss and have a low maximum crosstalk in the bandwidth of interest. The wavelength response of the bends is dependent on the inclusion of tapers, which manifests as a mild trade-off between excess loss and intermodal crosstalk. The optimized bend geometries were simulated with and without a 5 $\mu m$ taper, and results for the $340 \mathrm{~nm}$ bend TE and TM inputs are shown in Fig. 4.31 and Fig. 4.32, respectively. The results for the $220 \mathrm{~nm}$ bend variant are shown in Fig. 4.33. The maximum excess loss and crosstalk are compared in tables 4.2 and 4.3 . 

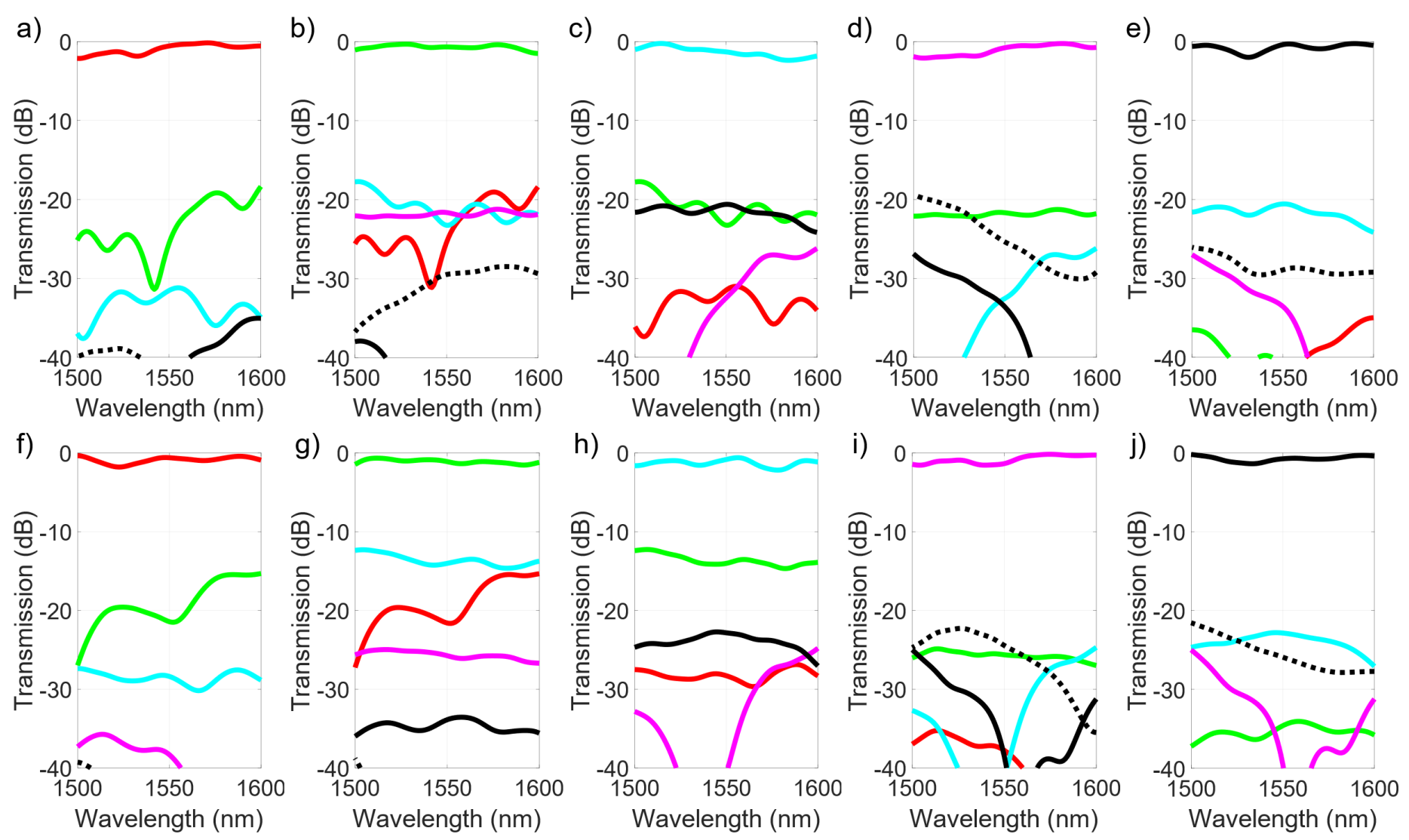

\section{-TE0 -TE1 -TE2 -TE3 -TE4 - -TM0 - -TM1 - "TM2 - -TM3 - -TM4}

Figure 4.31: Transmission spectra for a $340 \mathrm{~nm}$ bend without a taper: a) $\mathrm{TE}_{0}$, b) $\mathrm{TE}_{1}, \mathrm{c}$ ) $\mathrm{TE}_{2}$, d) $\mathrm{TE}_{3}$, e) $\mathrm{TE}_{4}$. With a $5 \mu m$ taper: f) $\mathrm{TE}_{0}$, g) $\left.\mathrm{TE}_{1}, \mathrm{~h}\right) \mathrm{TE}_{2}$, i) $\mathrm{TE}_{3}$, j) $\mathrm{TE}_{4}$. 


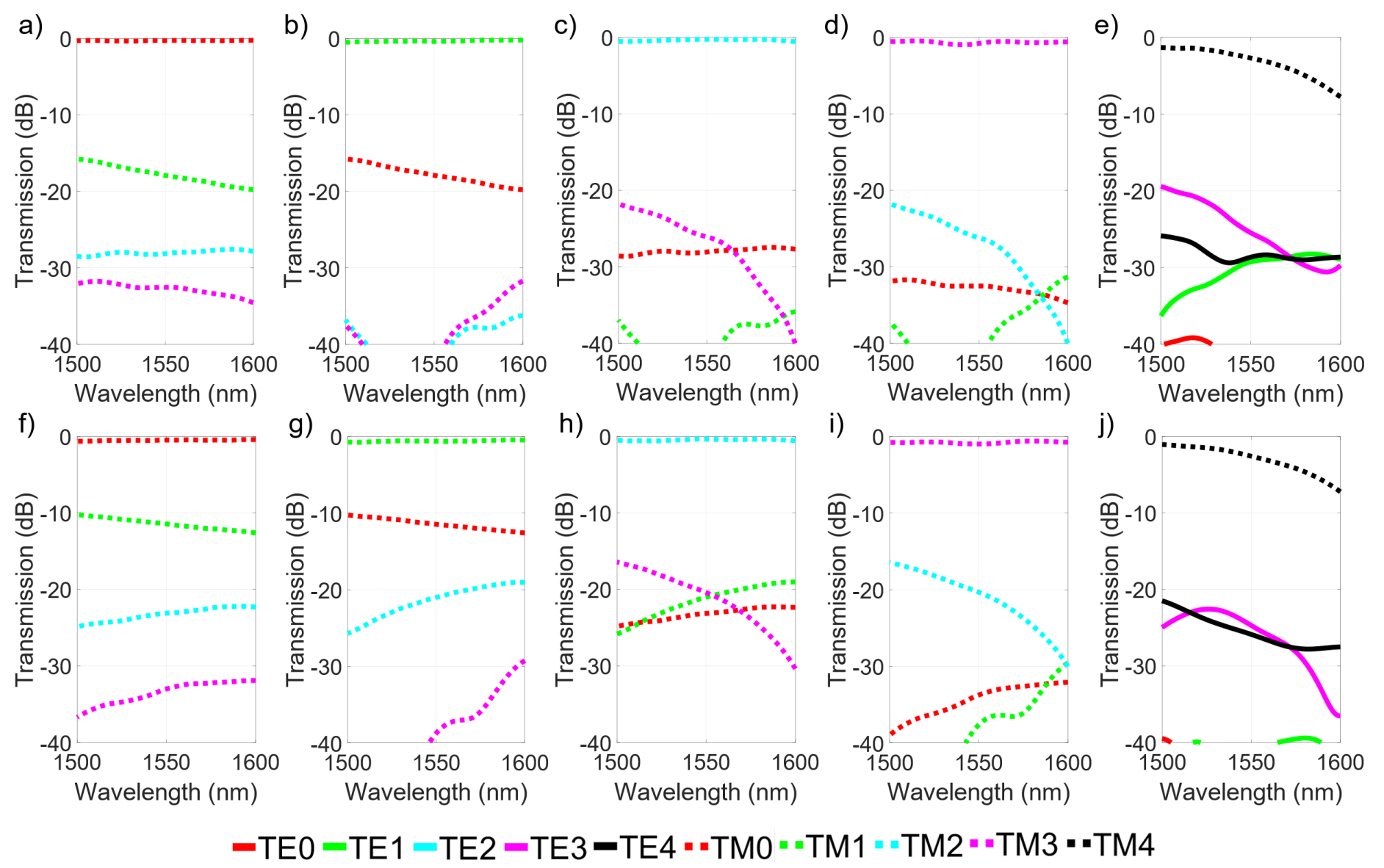

Figure 4.32: Transmission spectra for a $340 \mathrm{~nm}$ bend without a taper: a) $\mathrm{TM}_{0}$, b) $\mathrm{TM}_{1}$, c) $\mathrm{TM}_{2}$, d) $\mathrm{TM}_{3}$, e) $\mathrm{TM}_{4}$. With a $5 \mu m$ taper: f) $\mathrm{TM}_{0}$, g) $\mathrm{TM}_{1}$, h) $\mathrm{TM}_{2}$, i) $\mathrm{TM}_{3}$, j) $\mathrm{TM}_{4}$. 
4. SWG Engineered Multimode Waveguide Bends
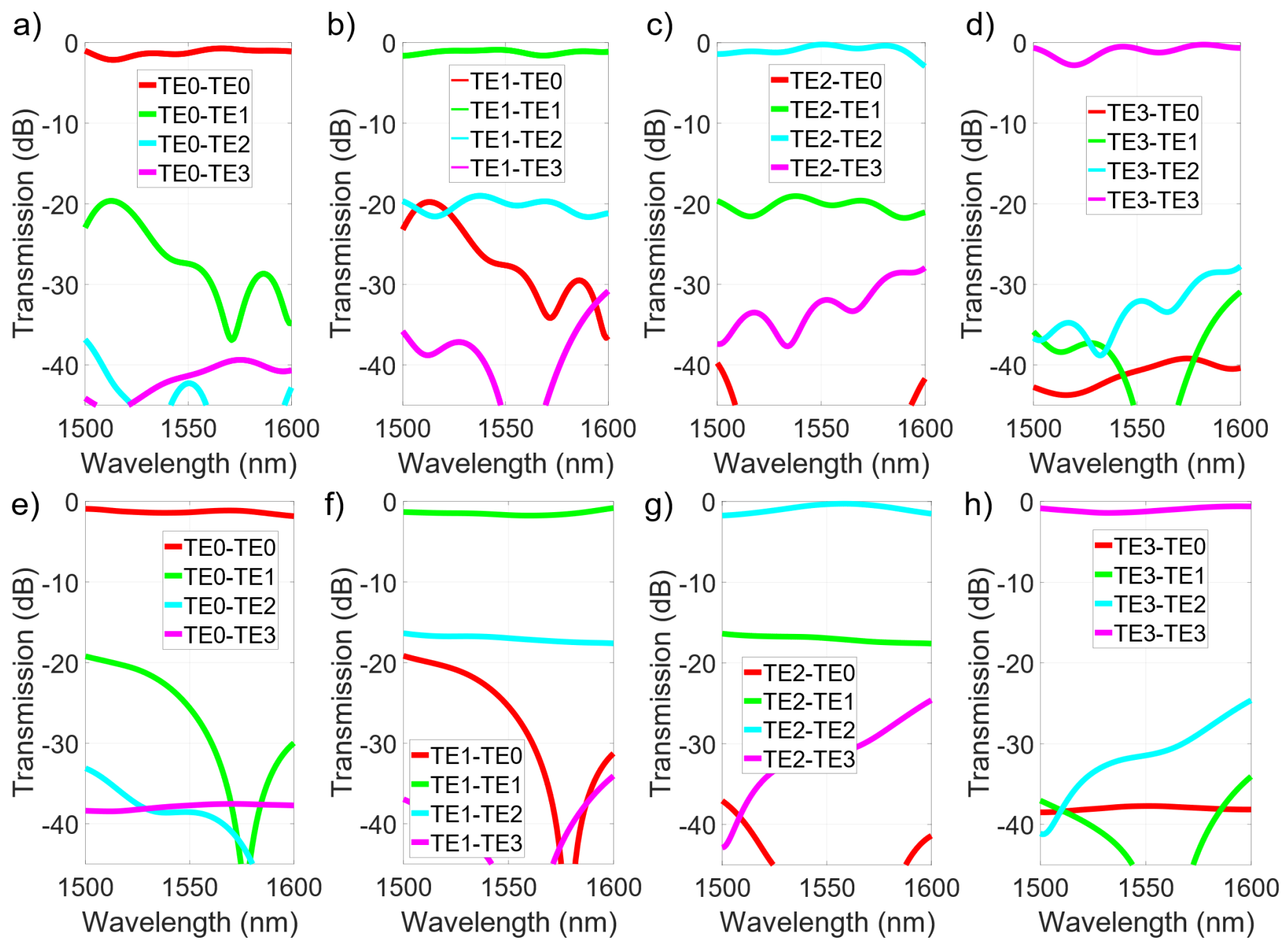

Figure 4.33: a) to d) Transmission spectra for the $\mathrm{TE}_{0-3}$ modes without a taper. e) to $\mathrm{h}$ ) Transmission spectra for the $\mathrm{TE}_{0-3}$ modes with a $5 \mu \mathrm{m}$ long taper. 
Table 4.2: Maximum excess loss and intermodal crosstalk for the $340 \mathrm{~nm}$ bend variant with and without a $5 \mu m$ taper.

\begin{tabular}{c|c|c|c|c}
\hline- & \multicolumn{2}{|c}{ Without Taper } & \multicolumn{2}{c}{ With Taper } \\
Mode & $\operatorname{Max} E L(\mathrm{~dB})$ & $\operatorname{Max} C T(\mathrm{~dB})$ & $\operatorname{Max} E L(\mathrm{~dB})$ & $\operatorname{Max} C T(\mathrm{~dB})$ \\
\hline $\mathrm{TE}_{0}$ & 2.13 & -18.35 & 1.79 & -15.32 \\
$\mathrm{TE}_{1}$ & 1.50 & -17.71 & 1.54 & -12.30 \\
$\mathrm{TE}_{2}$ & 2.37 & -17.74 & 2.18 & -12.27 \\
$\mathrm{TE}_{3}$ & 2.09 & -19.37 & 1.56 & -22.29 \\
$\mathrm{TE}_{4}$ & 1.99 & -20.56 & 1.37 & -21.54 \\
$\mathrm{TM}_{0}$ & 0.34 & -15.75 & 0.63 & -10.22 \\
$\mathrm{TM}_{1}$ & 0.48 & -15.74 & 0.75 & -10.26 \\
$\mathrm{TM}_{2}$ & 0.54 & -21.74 & 0.57 & -16.40 \\
$\mathrm{TM}_{3}$ & 0.94 & -21.76 & 0.98 & -16.46 \\
$\mathrm{TM}_{4}$ & 7.75 & -19.42 & 7.22 & -21.46 \\
\hline
\end{tabular}

Table 4.3: Maximum excess loss and intermodal crosstalk for the $220 \mathrm{~nm}$ bend variant with and without a $5 \mu m$ taper.

\begin{tabular}{c|c|c|c|c}
\hline- & \multicolumn{2}{|c}{ Without Taper } & \multicolumn{2}{c}{ With Taper } \\
Mode & Max $E L(\mathrm{~dB})$ & $\operatorname{Max} C T(\mathrm{~dB})$ & $\operatorname{Max} E L(\mathrm{~dB})$ & $\operatorname{Max} C T(\mathrm{~dB})$ \\
\hline $\mathrm{TE}_{0}$ & 2.14 & -19.65 & 1.82 & -19.22 \\
$\mathrm{TE}_{1}$ & 1.64 & -19.01 & 1.76 & -16.36 \\
$\mathrm{TE}_{2}$ & 2.96 & -19.05 & 1.75 & -16.37 \\
$\mathrm{TE}_{3}$ & 2.81 & -27.76 & 1.42 & -24.62 \\
\hline
\end{tabular}

It can be observed that the inclusion of a taper reduces the excess loss anywhere from 0.19-1.39 dB depending on the mode. However, this reduction in loss comes at the expense of an increase in the intermodal crosstalk, which can increase anywhere from 0.43-5.52 dB. In the case of the $220 \mathrm{~nm}$ bend the trade-off of increased crosstalk for reduced loss is reasonable, but less-so in the $340 \mathrm{~nm}$ case. Regardless of the inclusion of the taper the TM modes have a relatively low excess loss when compared to the TE modes. In fact in some cases the 
excess loss of the TM modes shows a marginal increase with the taper, in addition to a fairly high crosstalk of $-10.2 \mathrm{~dB}$. If low power loss is a critical design requirement an entirely TM-focused bend is worth investigating.

\subsection{Layout and Fabrication}

The $220 \mathrm{~nm}$ thick bend variant was fabricated by Applied Nanotools on the same wafer as the Bragg filter devices from section 3.7. Devices were patterned using $100 \mathrm{keV}$ electronbeam-lithography with a minimum silicon feature size of $60 \mathrm{~nm}$, followed by the application of an upper-cladding of $\mathrm{SiO}_{2}$ via chemical vapour deposition. To assess the effectiveness of the multimode bend, each mode order must be investigated separately to ensure proper transmission. Individual modes cannot be measured directly, but rather indirectly measured through a (de)MUX circuit, as depicted in Fig. 4.34. Four inputs are supplied via grating couplers, which excite the $\mathrm{TE}_{0}$ mode in the four input waveguides. These four inputs are multiplexed into a large bus waveguide using a series of carefully designed directional coupler sections. The geometry of the MUX is specified in table 4.4. This TE-polarization MUX design is based off of the work in [53], but uses only the first four mode channels. The minimum bend radius of the grating coupler branches is $25 \mu \mathrm{m}$, well above the threshold where $\mathrm{TE}_{0}$ input would be susceptible to radiation loss. 


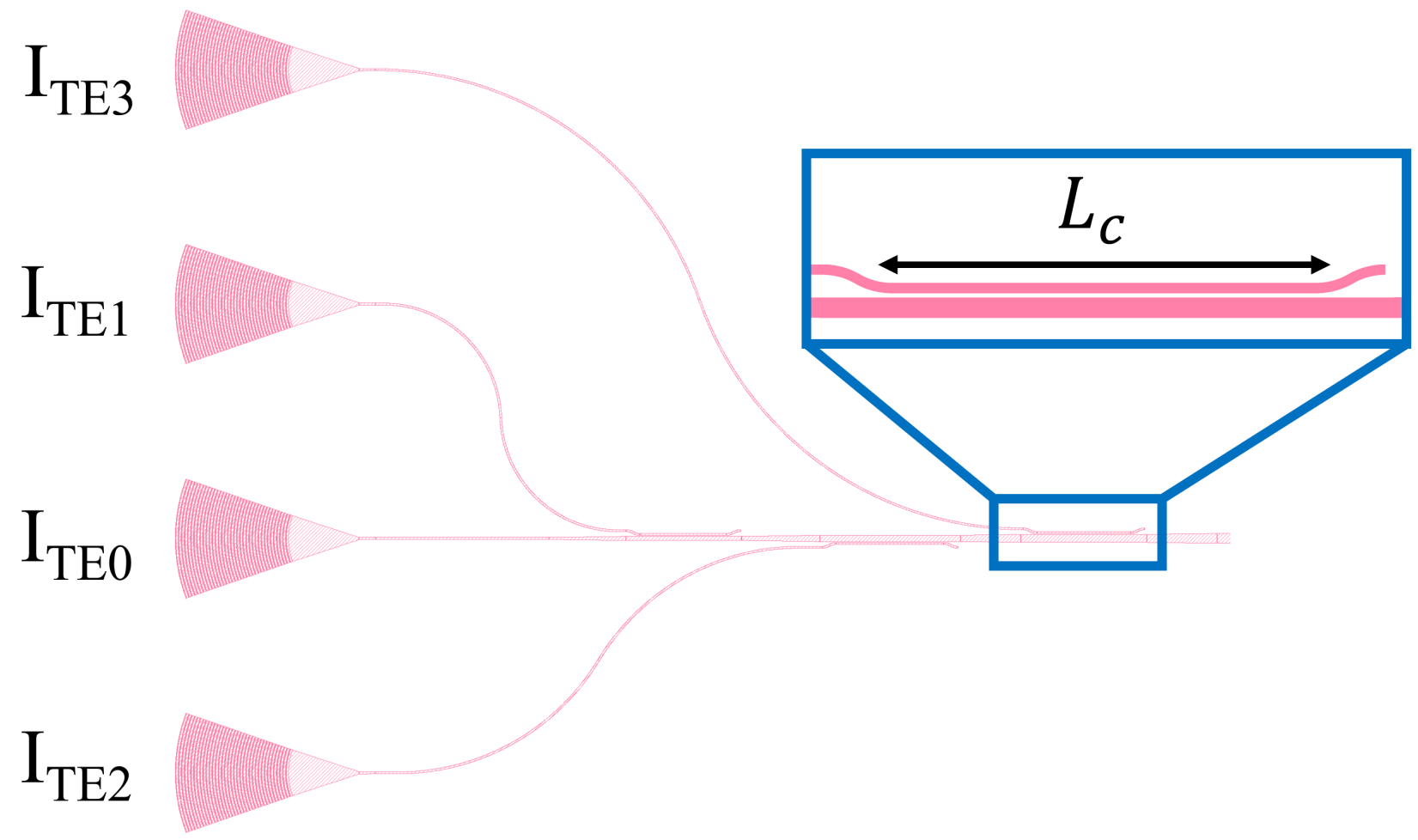

Figure 4.34: Layout diagram of one half of a (de)MUX circuit. It consists of four input grating couplers, each one coupling a different TE mode into the bus waveguide using adiabatic tapers. The multimode bends can be incorporated into this circuit using two in an S-bend configuration.

Table 4.4: Dimensions of the fabricated (de)MUX circuit.

\begin{tabular}{c|c|c|c|c|c}
\hline Mode & $W_{\text {access }}(\mu m)$ & $W_{\text {bus }}(\mu m)$ & Gap $(\mathrm{nm})$ & $L_{c}(\mu m)$ & Taper length $(\mu m)$ \\
\hline $\mathrm{TE}_{0}$ & - & 0.45 & - & - & - \\
$\mathrm{TE}_{1}$ & 0.439 & 0.875 & 190 & 18.5 & 16.5 \\
$\mathrm{TE}_{2}$ & 0.434 & 1.355 & 190 & 23.0 & 16.8 \\
$\mathrm{TE}_{3}$ & 0.405 & 1.705 & 190 & 20.0 & 12.9 \\
\hline
\end{tabular}

Following the bus waveguide is the desired device-under-test (DUT), i.e. two bends in an $\mathrm{S}$ configuration. The bends included on the layout are a standard circular bend, the 
bridged-SWG bend discussed in this work, and a straight waveguide as a reference. The DUT is followed by a (de)MUX circuit, which leads to 4 output grating couplers. The performance of the DUT can be measured by supplying a wavelength swept input to each input coupler, and measuring the output spectrum of each output coupler. If functioning correctly only the $\mathrm{TE}_{0}$ output should be excited by the $\mathrm{TE}_{0}$ input, and so forth. A series of grating coupler loops were included on the layout to subtract the wavelength dependence of the grating couplers from the transmission data.

\subsection{Testing \& Measurement Results}

An SEM image of a fabricated (de)MUX circuit is shown in Fig. 4.35, in addition to a closer look at the fabricated multimode bend. The (de)MUX circuits were tested by supplying a tunable laser source to one input grating coupler at a time using a cleaved $13^{\circ}$ fiber. The transmission response in the range of 1500-1600 nm was measured for each output grating coupler, for a total of 16 measurements per (de)MUX circuit. 


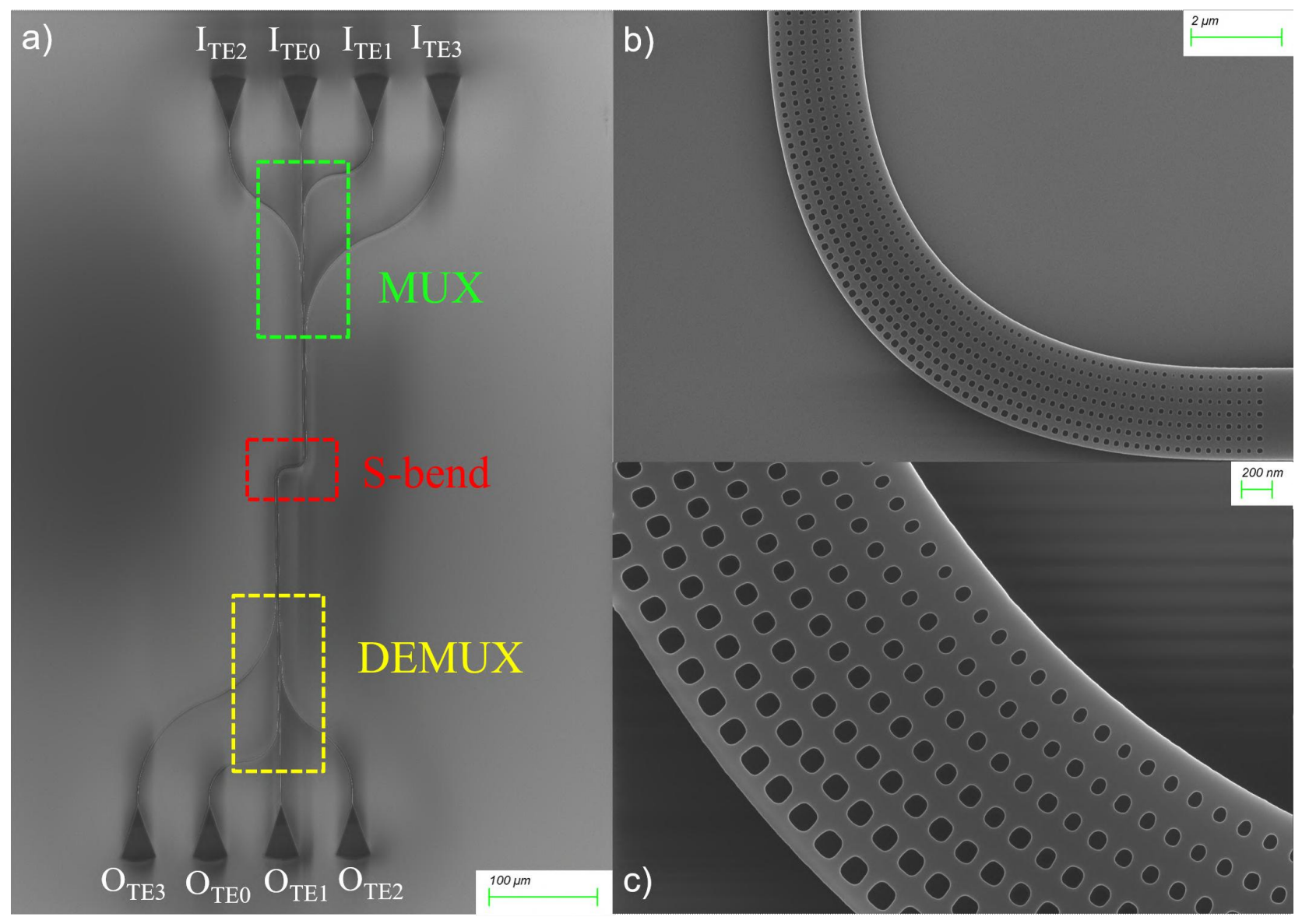

Figure 4.35: SEM photographs of the fabricated (de)MUX circuit for testing the multimode bend. a) The (de)MUX circuit, b) the multimode bend, and c) a zoomed-in picture of the bend.

\subsubsection{Grating Coupler Loopback Calibration}

A grating coupler loop was included on the wafer as a calibration circuit. The grating couplers were provided as part of Applied Nanotools' process design kit, and are optimized for the TE polarization, centered at $1550 \mathrm{~nm}$. The transmission spectra for one pair of grating couplers is shown in Fig. 4.36, where the response is parabolic with respect to wavelength. This 
wavelength dependence was fit with a polynomial function and was subsequently subtracted from all (de)MUX measurements in the following section.

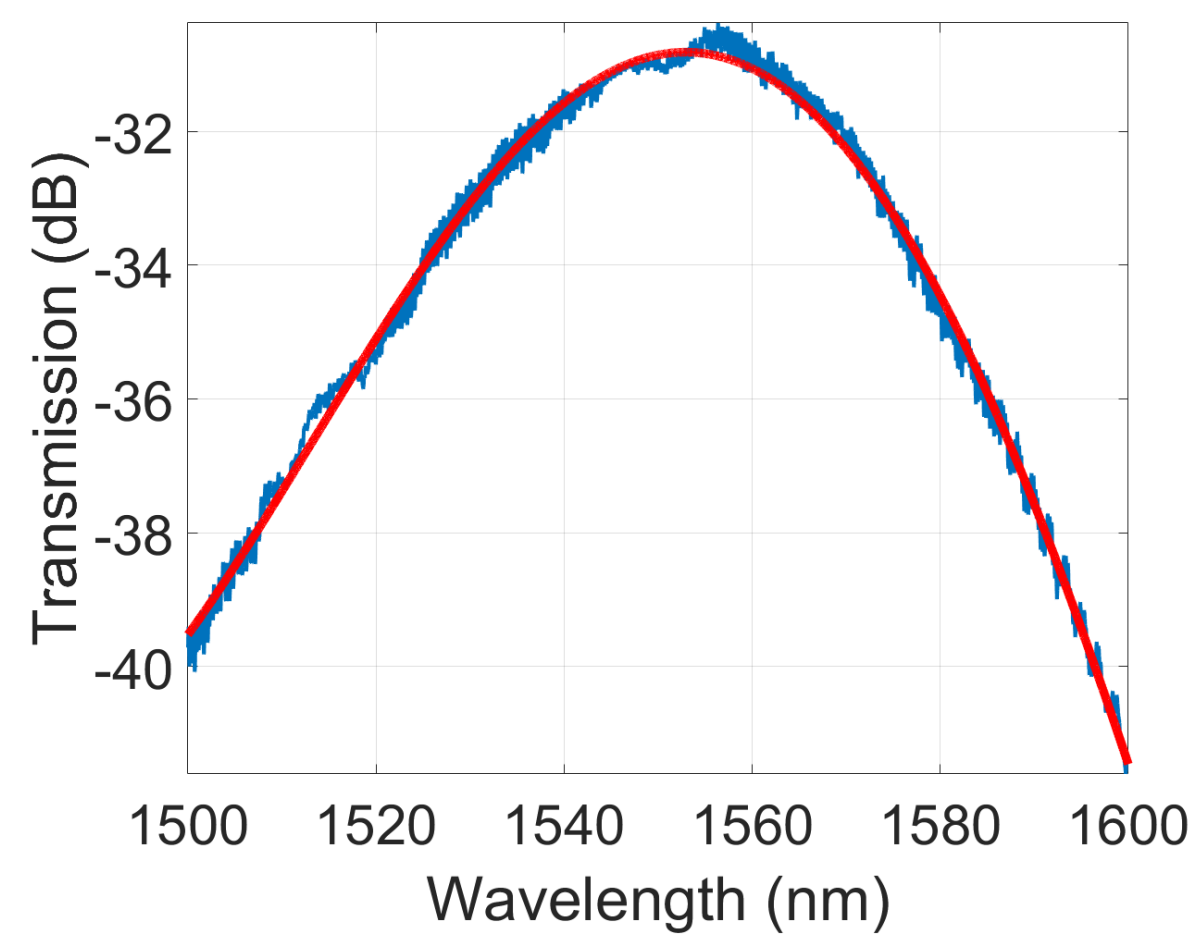

Figure 4.36: Transmission spectrum response for a grating coupler loop of length $1 \mathrm{~mm}$. Measured data is shown in blue and the curve fit is shown in red.

\subsection{2 (de)MUX Transmission Spectra}

Fig. 4.37 shows the measurement results for a (de)MUX circuit where the DUT is a straight waveguide, i.e. no bends. This circuit acts as the performance limit for the multimode bend design as there is a baseline level of crosstalk inherent to the (de)MUX circuit. The expected behaviour is observed, with each input mode exciting the corresponding mode at the output. The crosstalk separation between the desired mode and the next highest mode 
have minimum values of $15 \mathrm{~dB}, 11 \mathrm{~dB}, 11 \mathrm{~dB}$, and $10 \mathrm{~dB}$ for the $\mathrm{TE}_{0}, \mathrm{TE}_{1}, \mathrm{TE}_{2}, \mathrm{TE}_{3}$ modes, respectively.
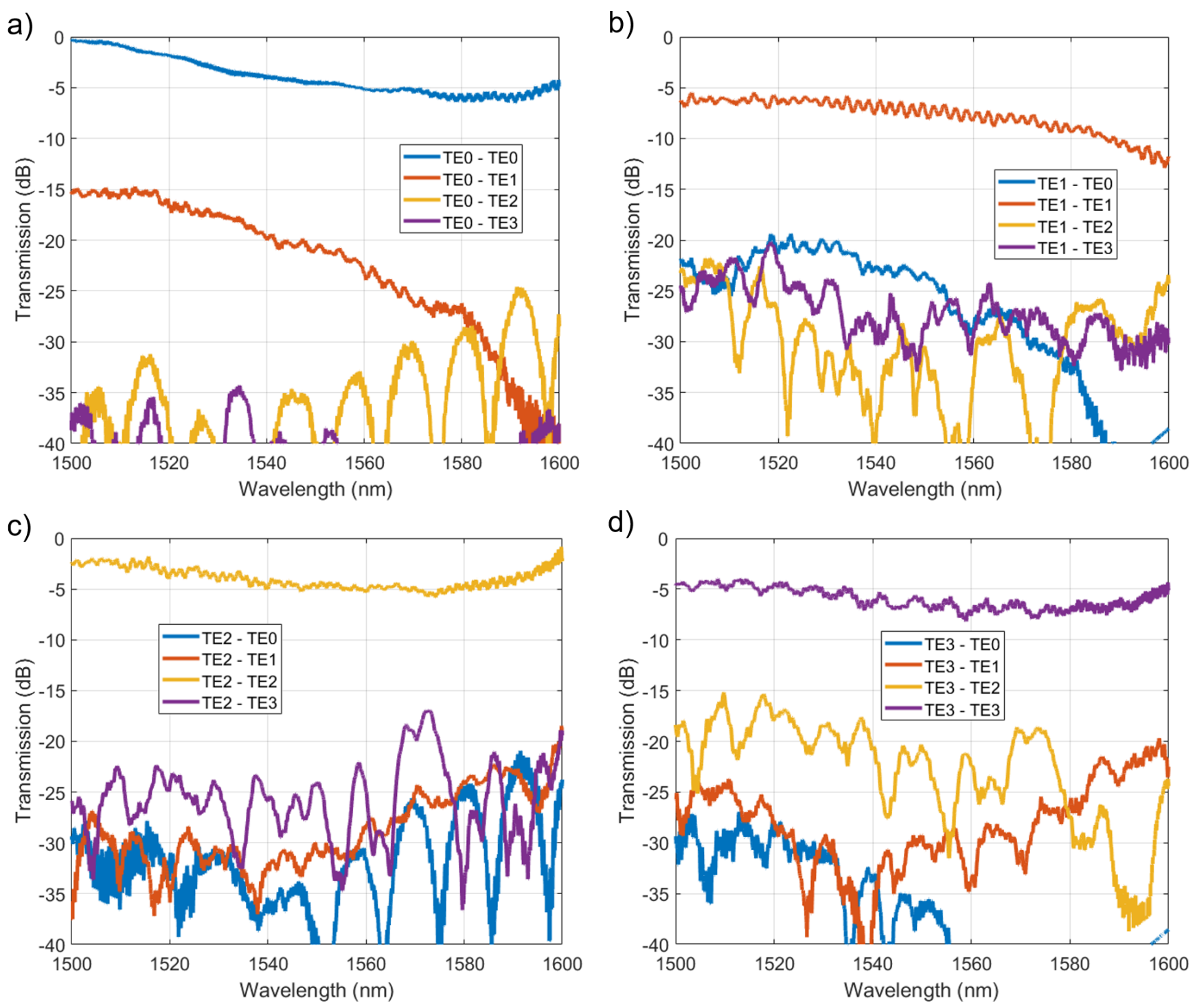

Figure 4.37: Measured transmission spectra for a (de)MUX with a straight waveguide: a) $\mathrm{TE}_{0}$, b) $\mathrm{TE}_{1}$, c) $\mathrm{TE}_{2}$, d) $\mathrm{TE}_{3}$.

Fig. 4.38 shows the measurement results for a (de)MUX circuit where the DUT is an 
S-bend consisting of two standard circular bends with a radius of $10 \mu \mathrm{m}$. It can be seen that the MUX cannot resolve between the various input modes, with the signals overlapping at random. When compared to the (de)MUX with no bends this circuit could not be used for MDM applications.
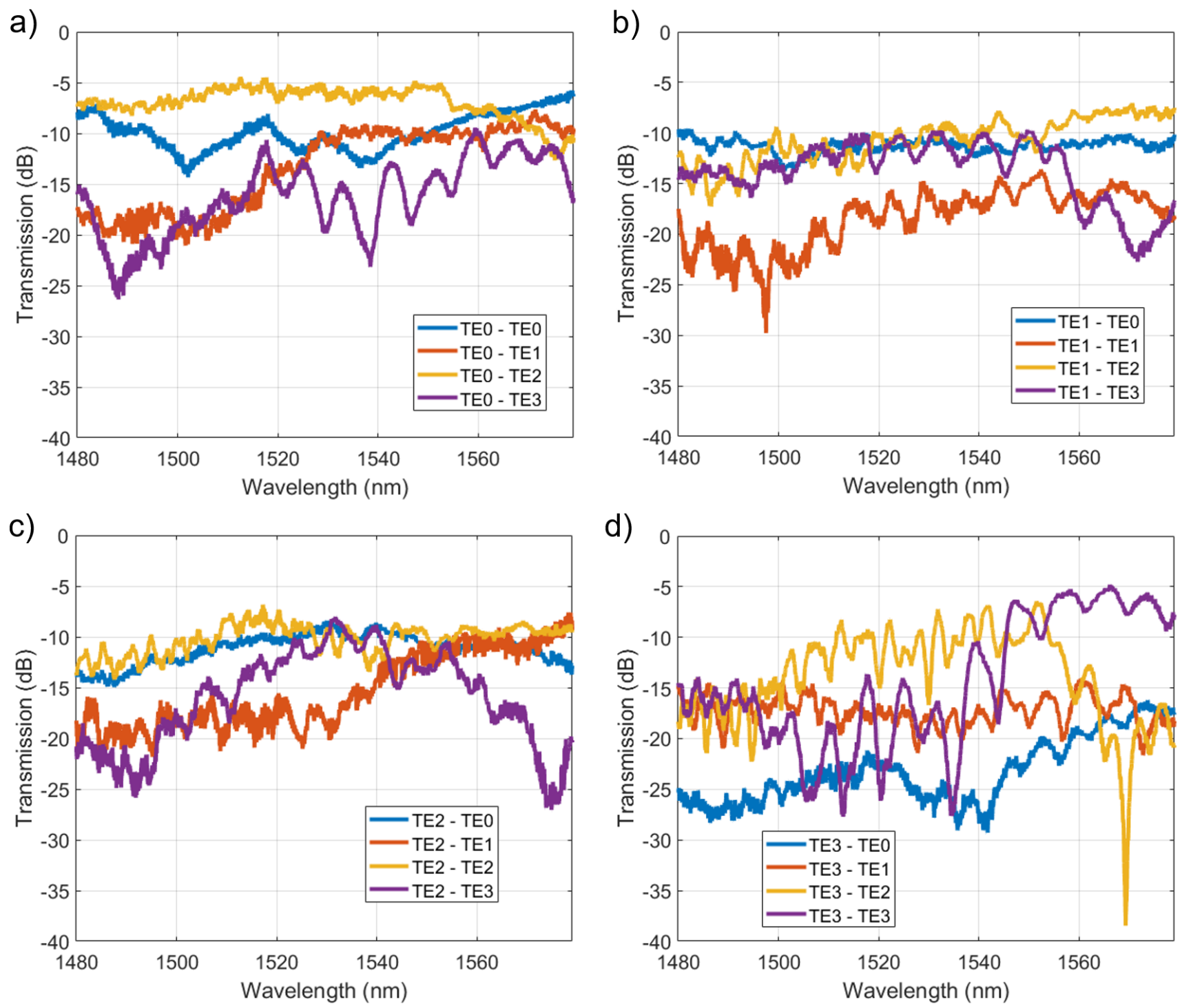

Figure 4.38: Measured transmission spectra for a (de)MUX with a standard circular bend: a) $\mathrm{TE}_{0}$, b) $\mathrm{TE}_{1}$, c) $\left.\mathrm{TE}_{2}, \mathrm{~d}\right) \mathrm{TE}_{3}$. 
Fig 4.39 shows the measurement results for a (de)MUX circuit where the DUT is an S-bend consisting of two SWG multimode bends with a radius of $10 \mu \mathrm{m}$ (no tapers were included on the fabrication run). It can been seen that the SWG multimode bend successfully resolves each desired mode order through the (de)MUX circuit. The average crosstalk values across the band for each mode are $-21.36 \mathrm{~dB},-14.39 \mathrm{~dB},-21.20 \mathrm{~dB}$ and $-16.34 \mathrm{~dB}$, for $\mathrm{TE}_{0}, \mathrm{TE}_{1}, \mathrm{TE}_{2}$, and $\mathrm{TE}_{3}$, respectively. When compared to the (de)MUX with a straight waveguide there are observable ripples in the transmission level, which was also observed in the simulated bends without tapers. This would likely be mitigated with the inclusion of the multimode taper section.

The multimode SWG bend shows significant improvement over the circular bends. A numerical comparison between the between the straight (de)MUX and the multimode bend (de)MUX is shown in table 4.5 in terms of the range of observed crosstalk values. The SWG multimode bend demonstrates a maximum crosstalk increase of 0.89-3.92 dB when compared to the straight waveguide, but has comparable minimum crosstalk.

Table 4.5: Performance comparison between (de)MUX with a straight waveguide and the SWG multimode bends.

\begin{tabular}{c|c|c|c|c}
\hline- & \multicolumn{2}{|c|}{ Straight Waveguide } & \multicolumn{2}{c}{ SWG Multimode S-Bend } \\
\hline Mode & Max CT (dB) & Min CT (dB) & Max CT (dB) & Min CT (dB) \\
\hline $\mathrm{TE}_{0}$ & -15.08 & -31.15 & -13.89 & -29.04 \\
$\mathrm{TE}_{1}$ & -11.48 & -21.51 & -7.56 & -21.05 \\
$\mathrm{TE}_{2}$ & -11.29 & -26.42 & -13.56 & -28.70 \\
$\mathrm{TE}_{3}$ & -10.07 & -23.41 & -9.18 & -21.18 \\
\hline
\end{tabular}


4. SWG Engineered Multimode Waveguide Bends

a)

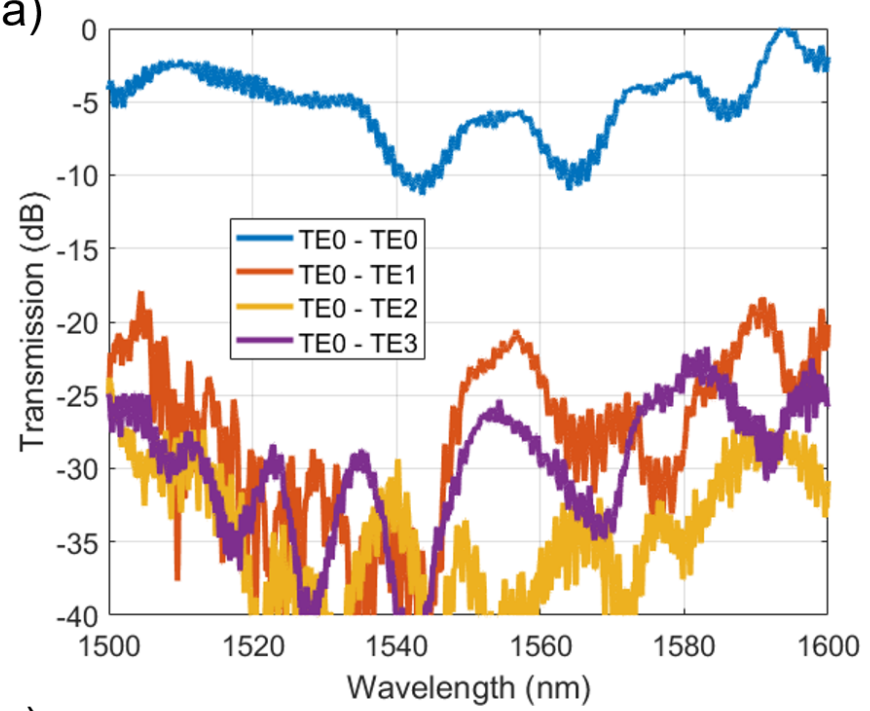

c)

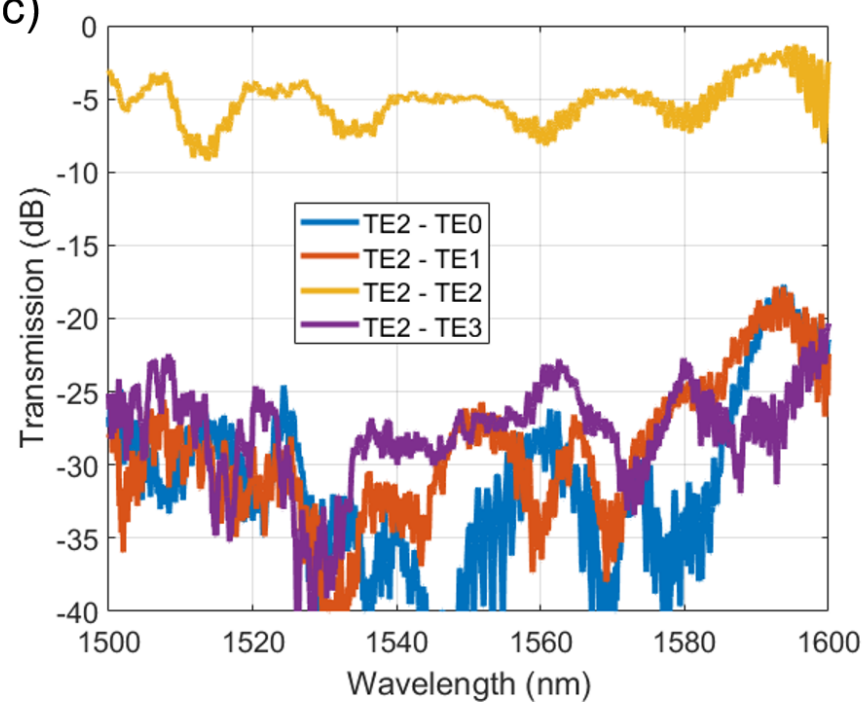

b)

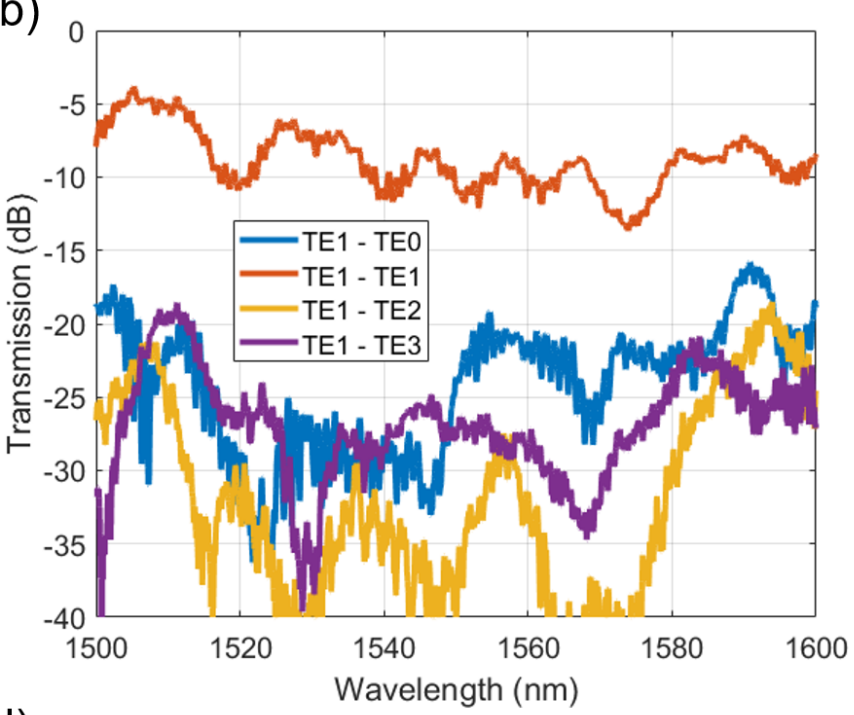

d)

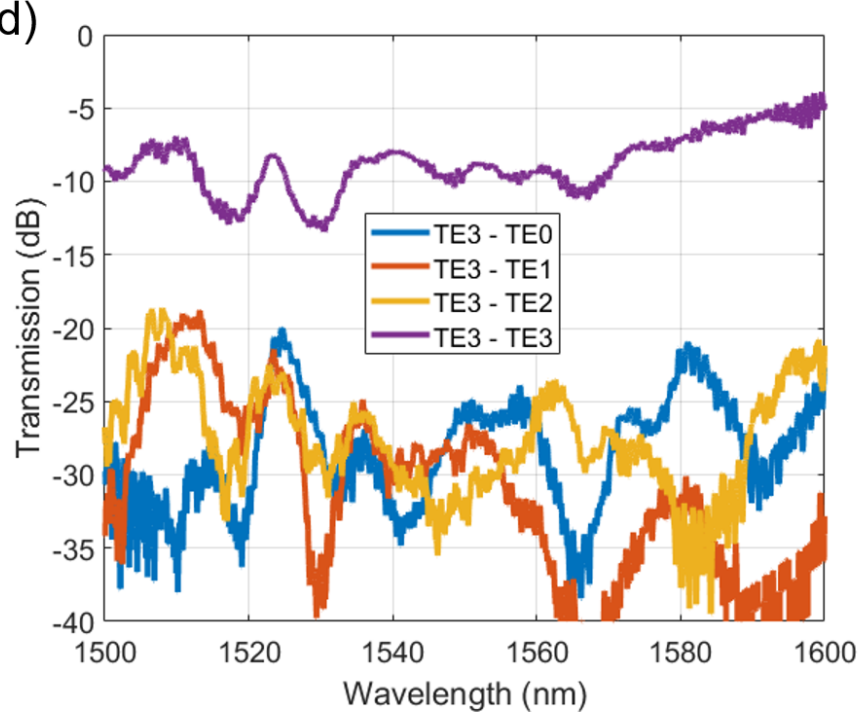

Figure 4.39: Measured transmission spectra for a (de)MUX with a multimode S-bend: a) $\left.\mathrm{TE}_{0}, \mathrm{~b}\right) \mathrm{TE}_{1}$, c) $\left.\mathrm{TE}_{2}, \mathrm{~d}\right) \mathrm{TE}_{3}$. 


\subsection{Summary}

This chapter has provided an overview of the design, simulation, fabrication and analysis of a fully-etched SOI multimode bend. Firstly, an overview of the basic theory of waveguide bends and their sources of loss was provided, as well as the state-of-the-art for silicon photonic multimode bends. This was followed by the introduction of a novel fully-etched multimode bend solution, consisting of trapezoidal SWG segments and radial bridging strips. An optimal curvature was calculated, and dozens of geometry variations were simulated in Lumerical FDTD, where the mover overlap was optimized. This bend was optimized for both the $340 \mathrm{~nm}$ and $220 \mathrm{~nm}$ SOI platforms, supporting $5 \mathrm{TE} / 4 \mathrm{TM}$ and 4 TE modes, respectively. The $220 \mathrm{~nm}$ SOI bend was fabricated and tested using a (de)MUX circuit, showing significant improvement over a standard circular bend. With the exception of the fabrication and testing, all work presented in this chapter was my own. 


\section{Chapter 5}

\section{Conclusion \& Future Work}

This thesis covered two distinct projects that involved the use of subwavelength gratings to enhance the potential capacity of WDM and MDM networks. Subwavelength gratings are extremely versatile and can be used for precise Bragg filter spectral design and index gradient tailoring for multimode bends.

\subsection{Bragg Filters}

The Bragg filters studied in this thesis consist of a core (either an SWG or wire) and a series of Bragg loading segments with variable $g(z)$. These filters can produce sub-nanometer bandwidths with just a constant value of $g_{0}$, but can be further modulated to produce arbitrarily complex spectral responses for specific WDM applications. A simple modulation scheme $g(z)$ that consists of the superposition of multiple cosines can be used to tune the extinction ratio and spacing of sidelobes with careful choice of modulation amplitudes and periods.

First it was shown that a single Bragg unit cell can be simulated to extract $\lambda_{B}$ and the bandwidth. These results can be used to derive a relationship between $g(z)$ and the coupling coefficient, and hence the index modulation $\Delta n(z)$, allowing for numerical FFTs to predict the filter response. These results were verified with full 3D FDTD simulations of a variety of 
filter combinations. Finally, the Bragg filters were fabricated and measured experimentally, confirming the previous analysis.

The spectra produced by the SWG filters are superior to the wire filters for two critical reasons. The first is that the spectra are less susceptible to phase jitter due to sidewall roughness, as less of the field profile is concentrated along the sidewalls. This produces peaks with a more uniform extinction ratio and shape. Secondly the SWG filters are better for engineering extremely narrowband filters, which would be ideal for designing dense frequency comb filters for WDM applications. This modulation scheme could be extended even further to any arbitrarily complex spectrum by mapping any reflection spectra to the coupling coefficient profile, and solving for the require spacing $g(z)$.

In addition to the passive modulation of $g(z)$, grating strength can be modulated via a periodic temperature gradient. This effect was investigated using finite-element simulations of a serpentine metallic heater. These temperature simulations were then imported into FDTD simulations of a grating with constant $g_{0}$ to investigate dynamic peak splitting as a function of supplied voltage. Although peak splitting was observed, the required temperature swings, and therefore voltages, were extremely high, and unlikely to be physically achievable. The fabricated devices with periodic heaters showed no observable peak splitting, and only demonstrated a shift in $\lambda_{B}$ due to a uniform temperature increase. Furthermore, the SWG filters were incompatible with the heaters as the lossy TM mode leaked upwards towards the metal heater.

To improve upon this work in the future, the focus should be on heater profiles with periods upwards of $1000 \Lambda_{B}$. This period would produce a frequency comb response with peak spacing in the sub-nanometer range, which is compatible with the sub-nanometer bandwidths that can be produced by the SWG filters in this work. Compatibility with the SWG filters 
5. Conclusion \& Future Work

would necessitate a larger upper cladding thickness to prevent leakage to the metal heater. Additionally, large heater periods allow for larger temperature swings for a given input voltage, with the stronger amplitude modulation resulting in a higher extinction ratio for the sidelobes. Because the larger periods require longer heaters it is worth investigating heater designs that are lower resistance. In addition to some geometric optimization, alternating between metallic interconnects and the metallic heater elements would likely reduce the resistance considerably.

\subsection{Multimode Bends}

The multimode bends studied in this thesis combine trapezoidal SWG segments with radial bridging strips to engineer a precise index gradient. This design allows for the first SWG multimode bend that can be fabricated with only one full etch step. This design affords multiple degrees of freedom when tailoring the index gradient, allowing for compact multimode bends necessary for dense MDM systems.

First the two waveguide heights of $220 \mathrm{~nm}$ and $340 \mathrm{~nm}$ were chosen and the dispersion relationship with respect to width was simulated for the first 10 modes. Following this a waveguide width of $2.075 \mu \mathrm{m}$ was chosen, as well as a target effective radius of $10 \mu \mathrm{m}$ and an SWG pitch of $220 \mathrm{~nm}$. These input parameters were used to calculated an optimized bend curve in Matlab before exporting the coordinate set to Lumerical FDTD for simulation sweeps. Dozens of radial strip widths were simulated for each mode order of interest, and the optimal geometry was that which maximized the mode overlap for each mode. The $220 \mathrm{~nm}$ bend was optimized for the first four TE modes, whereas the $340 \mathrm{~nm}$ bend was optimized for the first five TE and first four TM modes. Furthermore, this ideal bend 
geometry was simulated with and without the inclusion of a multimode taper section. The general conclusion being that a taper reduces the excess reflection losses at the expense of an increased intermodal crosstalk.

The $220 \mathrm{~nm}$ bend variant was experimentally demonstrated using a (de)MUX circuit of the first four TE modes. The multimode bend showed significant improvement when compared to a circular bend of the same radius, and comparable performance to a straight waveguide. To improve upon this work in the future, this design can be extended to support additional mode orders. In particular, a $220 \mathrm{~nm}$ thick bend could support potentially up to 10 mode orders with an increased width and radius. The $340 \mathrm{~nm}$ bend variant could similarly be reduced in size to focus entirely on the low-loss TM modes, or expanded in size to support additional TE and TM modes.

\subsection{Achievements \& Publications}

- Gary S. Duck Graduate Scholarship in Photonics (2020) and Ontario Graduate Scholarship (2020)

- Carleton University GRAD-Flix: 1-Minute Thesis Video Competition, $3^{\text {rd }}$ place and audience's choice (2021)

- Elected President, SPIE Carleton University Student Chapter (2021)

- Kevan K. MacKay, Winnie N. Ye, SOI multimode waveguide bend with radially bridged SWGs for broadband (de)multiplexing, EOS Annual Meeting, Rome, Sept $13^{\text {th }}, 2021$

- Kevan K. MacKay, Shurui Wang, Pavel Cheben, Winnie N. Ye, Subwavelength Grating Metamaterial Multimode Waveguide Bend for Single Etched Silicon Waveguide, Laser \& Photonics Reviews (submitted September, 2021) 


\section{List of References}

[1] "Cisco annual internet report (2018-2023)," https://www.cisco.com/c/en/us/solutions/ collateral/executive-perspectives/annual-internet-report/white-paper-c11-741490.pdf, accessed: 2021-06-28.

[2] H. Suzuki, M. Fujiwara, N. Takachio, K. Iwatsuki, T. Kitoh, and T. Shibata, "12.5 GHz spaced $1.28 \mathrm{tb} / \mathrm{s}$ (512-channel x $2.5 \mathrm{gb} / \mathrm{s}$ ) super-dense WDM transmission over $320 \mathrm{~km}$ SMF using multiwavelength generation technique," IEEE Photonics Technology Letters, vol. 14, no. 3, pp. 405-407, Mar. 2002. [Online]. Available: https://doi.org/10.1109/68.986828

[3] M. Singh and J. Malhotra, "Performance investigation of a $3.84 \mathrm{tb} / \mathrm{s}$ WDM-based FSO transmission system incorporating 3-d orthogonal modulation scheme," Photonic Network Communications, vol. 41, no. 2, pp. 177-188, Mar. 2021. [Online]. Available: https://doi.org/10.1007/s11107-021-00927-x

[4] G. Rademacher, B. J. Puttnam, R. S. Luis, T. A. Eriksson, N. K. Fontaine, M. Mazur, H. Chen, R. Ryf, D. T. Neilson, P. Sillard, F. Achten, Y. Awaji, and H. Furukawa, "1.01 peta-bit/s cl-band transmission over a 15-mode fiber," in 2020 European Conference on Optical Communications (ECOC). IEEE, Dec. 2020. [Online]. Available: https://doi.org/10.1109/ecoc48923.2020.9333321

[5] Optics and Photonics. National Academies Press, Feb. 2013. [Online]. Available: https://doi.org/10.17226/13491

[6] D. Miller, "Rationale and challenges for optical interconnects to electronic chips," Proceedings of the IEEE, vol. 88, no. 6, pp. 728-749, Jun. 2000. [Online]. Available: https://doi.org/10.1109/5.867687

[7] Q. Xu, P. Dong, and M. Lipson, "Breaking the delay-bandwidth limit in a photonic structure," Nature Physics, vol. 3, no. 6, pp. 406-410, Apr. 2007. [Online]. Available: https://doi.org/10.1038/nphys600 
[8] E. Li and A. X. Wang, "Theoretical analysis of energy efficiency and bandwidth limit of silicon photonic modulators," Journal of Lightwave Technology, vol. 37, no. 23, pp. 5801-5813, Dec. 2019. [Online]. Available: https://doi.org/10.1109/jlt.2019.2939775

[9] W. N. Ye and Y. Xiong, "Review of silicon photonics: history and recent advances," Journal of Modern Optics, vol. 60, no. 16, pp. 1299-1320, Sep. 2013. [Online]. Available: https://doi.org/10.1080/09500340.2013.839836

[10] R. Soref and B. Bennett, "Electrooptical effects in silicon," IEEE Journal of Quantum Electronics, vol. 23, no. 1, pp. 123-129, Jan. 1987. [Online]. Available: https://doi.org/10.1109/jqe.1987.1073206

[11] P. J. Bock, P. Cheben, J. H. Schmid, J. Lapointe, A. Delâge, S. Janz, G. C. Aers, D.-X. Xu, A. Densmore, and T. J. Hall, "Subwavelength grating periodic structures in silicon-on-insulator: a new type of microphotonic waveguide," Optics Express, vol. 18, no. 19, p. 20251, Sep. 2010. [Online]. Available: https://doi.org/10.1364/oe.18.020251

[12] K. O. Hill, Y. Fujii, D. C. Johnson, and B. S. Kawasaki, "Photosensitivity in optical fiber waveguides: Application to reflection filter fabrication," Applied Physics Letters, vol. 32, no. 10, pp. 647-649, May 1978. [Online]. Available: https://doi.org/10.1063/1.89881

[13] C. Li, D. Liu, and D. Dai, "Multimode silicon photonics," Nanophotonics, vol. 8, no. 2, pp. 227-247, Nov. 2018. [Online]. Available: https://doi.org/10.1515/nanoph-2018-0161

[14] S. D'Agostino, "Hertz's researches on electromagnetic waves," Historical Studies in the Physical Sciences, vol. 6, pp. 261-323, Jan. 1975. [Online]. Available: https://doi.org/10.2307/27757343

[15] S. M. Rytov, "Electromagnetic properties of a finely stratifed medium," Sov. Phys., vol. 2, no. 3, pp. 466-475, 1956.

[16] 2021. [Online]. Available: https://www.lumerical.com/products/\#device

[17] R. Loiacono, G. T. Reed, G. Z. Mashanovich, R. Gwilliam, S. J. Henley, Y. Hu, R. Feldesh, and R. Jones, "Laser erasable implanted gratings for integrated silicon photonics," Optics Express, vol. 19, no. 11, p. 10728, May 2011. [Online]. Available: https://doi.org/10.1364/oe.19.010728 
[18] Q. Fang, J. F. Song, X. Tu, L. Jia, X. Luo, M. Yu, and G. Q. Lo, "Carrier-induced silicon bragg grating filters with a p-i-n junction," IEEE Photonics Technology Letters, vol. 25, no. 9, pp. 810-812, May 2013. [Online]. Available: https://doi.org/10.1109/lpt.2013.2252611

[19] T. Murphy, J. Hastings, and H. Smith, "Fabrication and characterization of narrow-band bragg-reflection filters in silicon-on-insulator ridge waveguides," Journal of Lightwave Technology, vol. 19, no. 12, pp. 1938-1942, 2001. [Online]. Available: https://doi.org/10.1109/50.971688

[20] X. Wang, W. Shi, H. Yun, S. Grist, N. A. F. Jaeger, and L. Chrostowski, "Narrow-band waveguide bragg gratings on SOI wafers with CMOS-compatible fabrication process," Optics Express, vol. 20, no. 14, p. 15547, Jun. 2012. [Online]. Available: https://doi.org/10.1364/oe.20.015547

[21] X. Wang, W. Shi, R. Vafaei, N. A. F. Jaeger, and L. Chrostowski, "Uniform and sampled bragg gratings in SOI strip waveguides with sidewall corrugations," IEEE Photonics Technology Letters, 2011. [Online]. Available: https://doi.org/10.1109/lpt.2010.2103305

[22] D. T. H. Tan, K. Ikeda, and Y. Fainman, "Cladding-modulated bragg gratings in silicon waveguides," Optics Letters, vol. 34, no. 9, p. 1357, Apr. 2009. [Online]. Available: https://doi.org/10.1364/ol.34.001357

[23] P. Cheben, J. Čtyroký, J. H. Schmid, S. Wang, J. Lapointe, J. G. Wangüemert-Pérez, Í. Molina-Fernández, A. Ortega-Moñux, R. Halir, D. Melati, D. Xu, S. Janz, and M. Dado, "Bragg filter bandwidth engineering in subwavelength grating metamaterial waveguides," Optics Letters, vol. 44, no. 4, p. 1043, Feb. 2019. [Online]. Available: https://doi.org/10.1364/ol.44.001043

[24] M. Verbist, W. Bogaerts, and D. V. Thourhout, "Design of weak 1-d bragg grating filters in SOI waveguides using volume holography techniques," Journal of Lightwave Technology, vol. 32, no. 10, pp. 1915-1920, May 2014. [Online]. Available: https://doi.org/10.1109/jlt.2014.2316478

[25] M. W. Puckett, F. Vallini, A. Grieco, and Y. Fainman, "Multichannel bragg gratings in silicon waveguides with asymmetric sidewall modulation," Optics Letters, vol. 40, no. 3, p. 379, Jan. 2015. [Online]. Available: https://doi.org/10.1364/ol.40.000379 
[26] X. Wang, H. Yun, N. A. F. Jaeger, and L. Chrostowski, "Multi-period bragg gratings in silicon waveguides," in 2013 IEEE Photonics Conference. IEEE, Sep. 2013. [Online]. Available: https://doi.org/10.1109/ipcon.2013.6656627

[27] W. Zhang and J. Yao, "A fully reconfigurable waveguide bragg grating for programmable photonic signal processing," Nature Communications, vol. 9, no. 1, Apr. 2018. [Online]. Available: https://doi.org/10.1038/s41467-018-03738-3

[28] C. Klitis, M. Sorel, and M. J. Strain, "Active on-chip dispersion control using a tunable silicon bragg grating," Micromachines, vol. 10, no. 9, p. 569, Aug. 2019. [Online]. Available: https://doi.org/10.3390/mi10090569

[29] A. Ahuja, P. Steinvurzel, B. Eggleton, and J. Rogers, "Tunable single phase-shifted and superstructure gratings using microfabricated on-fiber thin film heaters," Optics Communications, vol. 184, no. 1-4, pp. 119-125, Oct. 2000. [Online]. Available: https://doi.org/10.1016/s0030-4018(00)00923-8

[30] Z. Wang and L. R. Chen, "A thermally tunable superstructure grating filter in silicon photonics," in 2018 IEEE Photonics Conference (IPC). IEEE, Sep. 2018. [Online]. Available: https://doi.org/10.1109/ipcon.2018.8527163

[31] X. Wang, Y. Wang, J. Flueckiger, R. Bojko, A. Liu, A. Reid, J. Pond, N. A. F. Jaeger, and L. Chrostowski, "Precise control of the coupling coefficient through destructive interference in silicon waveguide bragg gratings," Optics Letters, vol. 39, no. 19, p. 5519, Sep. 2014. [Online]. Available: https://doi.org/10.1364/ol.39.005519

[32] "Applied nanotools - nanosoi process specifications," https://www.appliednt.com/ nanosoi/sys/resources/specs/, accessed: 2021-07-12.

[33] J. Čtyroký, J. G. Wangüemert-Pérez, P. Kwiecien, I. Richter, J. Litvik, J. H. Schmid, Í. Molina-Fernández, A. Ortega-Moñux, M. Dado, and P. Cheben, "Design of narrowband bragg spectral filters in subwavelength grating metamaterial waveguides," Optics Express, vol. 26, no. 1, p. 179, Jan. 2018. [Online]. Available: https://doi.org/10.1364/oe.26.000179 
[34] M. Heiblum and J. Harris, "Analysis of curved optical waveguides by conformal transformation," IEEE Journal of Quantum Electronics, vol. 11, no. 2, pp. 75-83, Feb. 1975. [Online]. Available: https://doi.org/10.1109/jqe.1975.1068563

[35] M. Bahadori, M. Nikdast, Q. Cheng, and K. Bergman, "Universal design of waveguide bends in silicon-on-insulator photonics platform," Journal of Lightwave Technology, vol. 37, no. 13, pp. 3044-3054, Jul. 2019. [Online]. Available: https://doi.org/10.1109/jlt.2019.2909983

[36] D. E. Hagan and A. P. Knights, "Mechanisms for optical loss in SOI waveguides for mid-infrared wavelengths around 2m," Journal of Optics, vol. 19, no. 2, p. 025801, Dec. 2016. [Online]. Available: https://doi.org/10.1088/2040-8986/19/2/025801

[37] C. Sun, Y. Yu, G. Chen, and X. Zhang, "Ultra-compact bent multimode silicon waveguide with ultralow inter-mode crosstalk," Optics Letters, vol. 42, no. 15, p. 3004, Jul. 2017. [Online]. Available: https://doi.org/10.1364/ol.42.003004

[38] M. Teng, A. A. Noman, Y. J. Lee, Z. Kong, Y. Xuan, and M. Qi, "A 3-micron-radius bend for SOI TE0/TE1 multiplexing," in Conference on Lasers and Electro-Optics. OSA, 2018. [Online]. Available: https://doi.org/10.1364/cleo_at.2018.jw2a.13

[39] W. Chang, L. Lu, D. Liu, and M. Zhang, "Ultra-compact silicon multi-mode waveguide bend based on subwavelength asymmetric y-junction," in Optical Fiber Communication Conference. OSA, 2018. [Online]. Available: https://doi.org/10.1364/ofc.2018.tu3a.1

[40] H. Xu and Y. Shi, "Ultra-sharp multi-mode waveguide bending assisted with metamaterial-based mode converters," Laser 83 Photonics Reviews, vol. 12, no. 3, p. 1700240, Jan. 2018. [Online]. Available: https://doi.org/10.1002/lpor.201700240

[41] Y. Wang and D. Dai, "Multimode silicon photonic waveguide corner-bend," Optics Express, vol. 28, no. 7, p. 9062, Mar. 2020. [Online]. Available: https://doi.org/10.1364/oe.387978

[42] B. A. Dorin and W. N. Ye, "Two-mode division multiplexing in a silicon-on-insulator ring resonator," Optics Express, vol. 22, no. 4, p. 4547, Feb. 2014. [Online]. Available: https://doi.org/10.1364/oe.22.004547 
[43] X. Jiang, H. Wu, and D. Dai, "Low-loss and low-crosstalk multimode waveguide bend on silicon," Optics Express, vol. 26, no. 13, p. 17680, Jun. 2018. [Online]. Available: https://doi.org/10.1364/oe.26.017680

[44] M. Cherchi, S. Ylinen, M. Harjanne, M. Kapulainen, and T. Aalto, "Dramatic size reduction of waveguide bends on a micron-scale silicon photonic platform," Optics Express, vol. 21, no. 15, p. 17814, Jul. 2013. [Online]. Available: https://doi.org/10.1364/oe.21.017814

[45] Y. Wang and D. Dai, "Ultra-sharp multimode waveguide bends with dual polarizations," Journal of Lightwave Technology, pp. 1-1, 2020. [Online]. Available: https://doi.org/10.1109/jlt.2020.2982736

[46] T. Fujisawa, S. Makino, T. Sato, and K. Saitoh, "Low-loss, compact, and fabrication-tolerant si-wire $90^{\circ}$ waveguide bend using clothoid and normal curves for large scale photonic integrated circuits," Optics Express, vol. 25, no. 8, p. 9150, Apr. 2017. [Online]. Available: https://doi.org/10.1364/oe.25.009150

[47] J. H. Song, T. D. Kongnyuy, P. D. Heyn, S. Lardenois, R. Jansen, and X. Rottenberg, "Low-loss waveguide bends by advanced shape for photonic integrated circuits," Journal of Lightwave Technology, vol. 38, no. 12, pp. 3273-3279, Jun. 2020. [Online]. Available: https://doi.org/10.1109/jlt.2020.2978420

[48] L. H. Gabrielli, D. Liu, S. G. Johnson, and M. Lipson, "On-chip transformation optics for multimode waveguide bends," Nature Communications, vol. 3, no. 1, Jan. 2012. [Online]. Available: https://doi.org/10.1038/ncomms2232

[49] Z. Wang, X. Xu, D. Fan, Y. Wang, H. Subbaraman, and R. T. Chen, "Geometrical tuning art for entirely subwavelength grating waveguide based integrated photonics circuits," Scientific Reports, vol. 6, no. 1, May 2016. [Online]. Available: https://doi.org/10.1038/srep24106

[50] H. Wu, C. Li, L. Song, H.-K. Tsang, J. E. Bowers, and D. Dai, "Ultra-sharp multimode waveguide bends with subwavelength gratings," Laser 83 Photonics Reviews, vol. 13, no. 2, p. 1800119, Jan. 2019. [Online]. Available: https: //doi.org/10.1002/lpor.201800119 
[51] D. Gonzalez-Andrade, J. G. Wanguemert-Perez, A. Velasco, A. Ortega-Monux, A. Herrero-Bermello, I. Molina-Fernandez, R. Halir, and P. Cheben, "Ultrabroadband mode converter and multiplexer based on sub-wavelength structures," IEEE Photonics Journal, vol. 10, no. 2, pp. 1-10, Apr. 2018. [Online]. Available: https://doi.org/10.1109/jphot.2018.2819364

[52] Z. Ruan, L. Shen, S. Zheng, and J. Wang, "Subwavelength grating slot (SWGS) waveguide on silicon platform," Optics Express, vol. 25, no. 15, p. 18250, Jul. 2017. [Online]. Available: https://doi.org/10.1364/oe.25.018250

[53] Y. He, Y. Zhang, Q. Zhu, S. An, R. Cao, X. Guo, C. Qiu, and Y. Su, "Silicon high-order mode (de)multiplexer on single polarization," Journal of Lightwave Technology, vol. 36, no. 24, pp. 5746-5753, Dec. 2018. [Online]. Available: https://doi.org/10.1109/jlt.2018.2878529 